$12-1-2006$

\title{
Dynamic Analysis of Bridges Under Moving Loads
}

Sanjay B. Gurav

How does access to this work benefit you? Let us know!

Follow this and additional works at: https://commons.und.edu/theses

\section{Recommended Citation}

Gurav, Sanjay B., "Dynamic Analysis of Bridges Under Moving Loads" (2006). Theses and Dissertations. 2695.

https://commons.und.edu/theses/2695

This Dissertation is brought to you for free and open access by the Theses, Dissertations, and Senior Projects at UND Scholarly Commons. It has been accepted for inclusion in Theses and Dissertations by an authorized administrator of UND Scholarly Commons. For more information, please contact und.commons@library.und.edu. 


\title{
DYNAMIC ANALYSIS OF BRIDGES UNDER MOVING LOADS
}

\author{
by
}

Sanjay B. Gurav

Bachelor of Civil Engineering, Pune University, 1997

Master of Technology, Visvesvaraya Regional College of Engineering, Nagpur, 2000

\author{
A Dissertation \\ Submitted to the Graduate Faculty \\ of the \\ University of North Dakota \\ in partial fulfillment of the requirements
}

for the degree of

Doctor of Philosophy

Grand Forks, North Dakota

December

2006 

This dissertation, submitted by Sanjay B. Gurav in partial fulfillment of the requirements for the Degree of Doctor of Philosophy from the University of North Dakota, has been read by the Faculty Advisory Committee under whom the work has been done and is hereby approved.
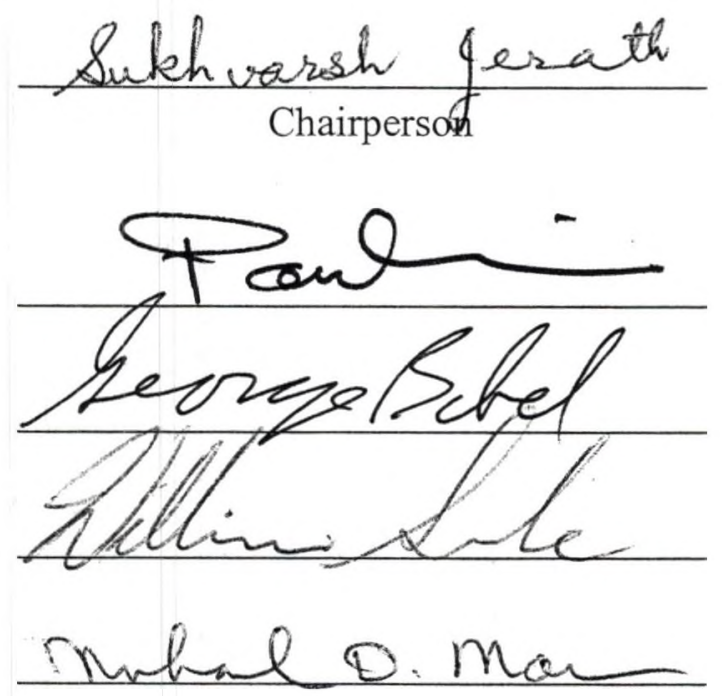

This dissertation meets the standards for apperance, conforms to the style and format requirements of the Graduate School of the University of North Dakota, and is hereby approved.

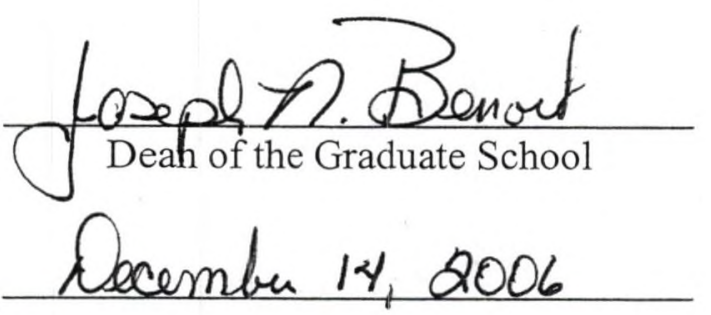

Date 


\section{PERMISSION}

Title Dynamic Analysis of Bridges under Moving Loads

\section{Department Engineering}

Degree Doctor of Philosophy

In presenting this dissertation in partial fulfillment of the requirements for a graduate degree from the University of North Dakota, I agree that the library of this University shall make it freely available for inspection. I further agree that permission for extensive copying for scholarly purposes may be granted by the professor who supervised my dissertation work or, in his absence, by the chairperson of the department or the dean of the Graduate School. It is understood that any copying or publication or other use of this dissertation or part thereof for financial gain shall not be allowed without my written permission. It is also understood that due recognition shall be given to me and to the University of North Dakota in any scholarly use which may be made of any material in my dissertation.

Signature

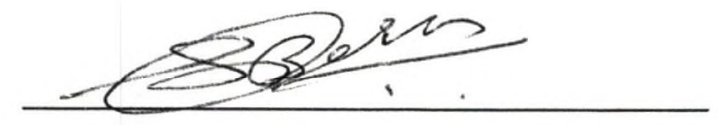

Date $11 / 30 / 06$ 


\section{TABLE OF CONTENTS}

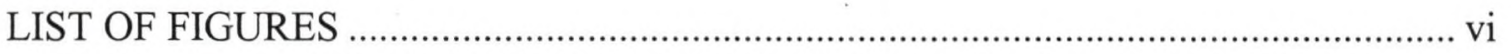

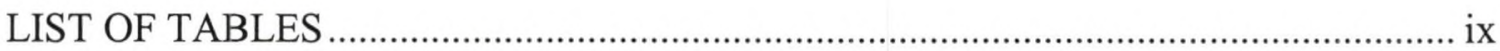

ACKNOWLEDGEMENTS .................................................................................

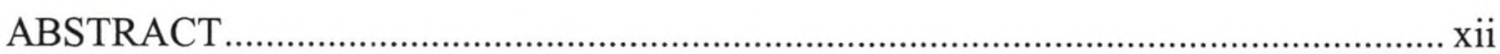

\section{CHAPTER}

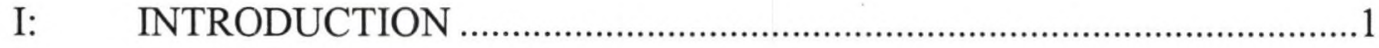

I-a: General Description...........................................................

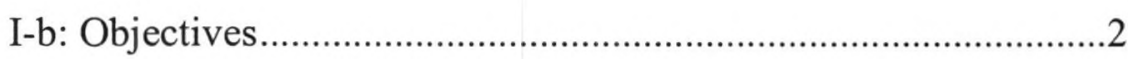

II: $\quad$ LITERATURE REVIEW ...................................................................

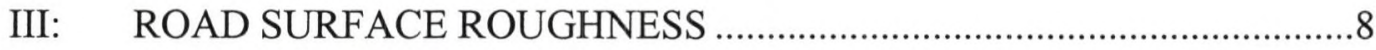

III-a: Power Spectral Density Function ........................................8

III-b: Generation of Road Surface Roughness............................14

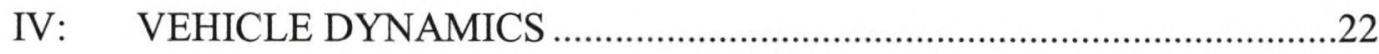

IV-a: 12 DOF Model of an AASHTO HS20-44 Truck .................22

IV-b: Suspension and Tire Forces of an

AASHTO HS20-44 Truck ..................................................32

V: $\quad$ ANALYSIS OF AN ORTHOTROPIC PLATE UNDER MOVING

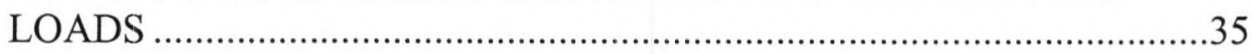

V-a: Generation of Equation of Motion...........................................35

V-b: Eigen-value Analysis of an Orthotropic plate ........................40 
VI: FINITE ELEMENT ANALYSIS OF AN ORTHOTROPIC PLATE UNDER MOVING LOADS

VII: ANALYSIS OF A T-BEAM BRIDGE UNDER A MOVING AASHTO HS20-44 TRUCK LOAD

VII-a: Generation of Road Surface Roughness for the T-beam Bridge Deck

VII-b: Suspension and Tire Forces of an AASHTO HS20-44 Truck

VII-c: Analysis of the T-beam Bridge Deck by using the Orthotropic Plate Theory .....

VII-d: Analysis of the T-beam Bridge Deck by using the Finite Element Analysis Method

VIII: CONCLUSIONS AND RECOMMENDATIONS 108

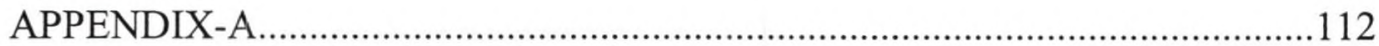

APPENDIX-B

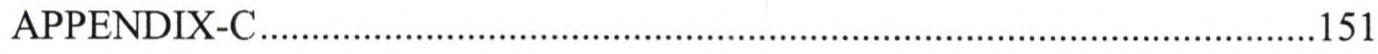

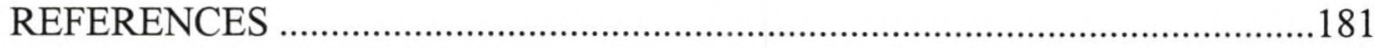




\section{LIST OF FIGURES}

Figure

Page

1 Spectrum of random numbers. .15

2 Comparison of Power Spectral Density of a random spectrum with the approximate Power Spectral Density.

3 Output from the first order digital filter.

4 Comparison of PSD of the output spectrum with the PSD from C.J.Dodds et.at..18

5 Typical road surface profile of a very good road

6 Typical road surface profile of a good road

7 Comparison of Power Spectral Density of a very good road surface with the approximate Power Spectral Density given by C. J. Dodds and J. D. Robson [3].20

8 Comparison of Power Spectral Density of a good road surface with the approximate Power Spectral Density given by C. J. Dodds and J. D. Robson [3].21

9 Side view of HS20-44 vehicle model

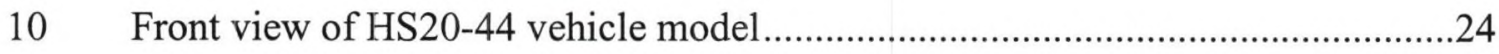

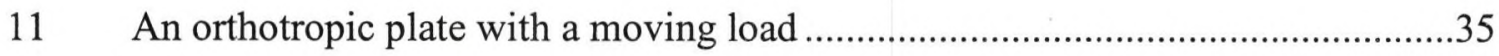

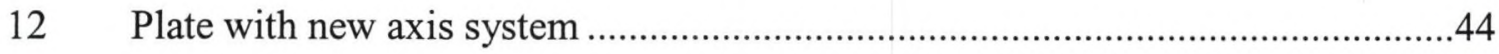

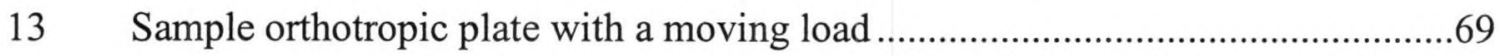

14 Vertical displacement history at the center of the plate with a load of 20,000 lb moving at the speed of $36 \mathrm{ft} / \mathrm{sec}(24.69 \mathrm{mph})$ 
15 Vertical displacement history at the center of the plate with a load of 20,000 lb moving at the speed of $72 \mathrm{ft} / \mathrm{sec}(49.39 \mathrm{mph})$.

16 Comparison of the vertical displacement at the center of the first plate at the speed of $36 \mathrm{ft} / \mathrm{sec}(24.69 \mathrm{mph})$

17 Comparison of the vertical displacement at the center of the second plate at the speed of $36 \mathrm{ft} / \mathrm{sec}(24.69 \mathrm{mph})$.

18 Element of T-beam bridge deck

19 Simply supported bridge considered in the investigation

20 Time history of vertical displacement at the center of the T-beam bridge deck under a single moving load.

21 Comparison of maximum vertical displacement at the center of T-beam bridge deck at different speeds under a single moving load

22 Time history of vertical displacement at the center of the T-beam bridge deck under two moving loads.....

23 Comparison of maximum vertical displacement at the center of T-beam bridge deck at different speeds under two moving loads....

24 A sample orthotropic plate with a moving load

25 Finite element model of isotropic plate by using 3D shell elements in NISA.....

26 Time history of vertical displacements at the center of the plate with a load of magnitude 20,000 lb moving at the speed of $36 \mathrm{ft} / \mathrm{sec}$ in NISA

27 Time history of vertical displacements at the center of the plate with a load of magnitude 20,000 lb moving at the speed of $72 \mathrm{ft} / \mathrm{sec}$ in NISA

28 Time history of vertical displacements at the center of the T-beam bridge deck under a single moving load in NISA

29 Comparison of maximum vertical displacement at the center of T-beam bridge deck at different speeds under a single moving load in NISA.

30 Time history of vertical displacements at the center of the T-beam bridge deck under two moving loads in NISA

31 Comparison of maximum vertical displacement at the center of T-beam bridge deck at different speeds under two moving loads in NISA 
32 Simply supported bridge considered in the investigation ....................................96

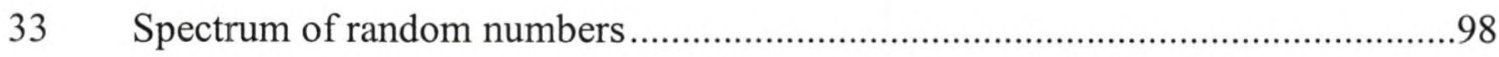

34 Comparison of Power Spectral Density of a random spectrum with the approximate Power Spectral Density......................................................................98

35 Road surface roughness of a very good road for the T-beam bridge deck ............99

36 Comparison of the PSD of the road surface roughness generated in MATLAB with the PSD given by C. J. Dodds and J. D. Robson [3] .................................100

37 Comparison of the vertical displacement under a moving AASHTO HS20-44 truck load with the static displacement by the orthotropic plate theory ..............104

38 Comparison of the vertical displacement under a moving AASHTO HS20-44 truck load with the static displacement by FEM.

39 Comparison of maximum vertical displacements at the center of the T-beam bridge deck under an AASHTO HS20-44 truck moving with different speeds by the orthotropic plate theory and by the finite element analysis method 


\section{LIST OF TABLES}

1 Spectrum constants according to classification of roads, MIRA [10]

2 Comparison of suspension forces at different speeds for HS20-44 truck on a very good road surface

3 Comparison of tire forces at different speeds for HS20-44 truck on a very good road surface.

4 Comparison of suspension forces at different speeds for HS20-44 truck on a good road surface.

5 Comparison of tire forces at different speeds for HS20-44 truck on a good road surface.

6 Comparison of the first four eigen-values with the eigen-values derived by Gorman D. J. [14]

7 Comparison of the first four eigen-values with the frequency parameters derived by Jayaraman et. al.

8 Categorization of orthotropic plates in accordance with the rigidity ratios

9 First four eigen-values of an orthotropic plate for different rigidity ratios. .70

10 Categories of orthotropic plates with different rigidity ratios for the first four mode shapes.

11 Comparison of maximum vertical displacements with the displacements investigated by Jong-shyong Wu et. al. [15].

12 First four eigen-values of the plate with $\frac{D_{x} / D_{y}=0.5 \& .}{H} / D_{y}=2$

13 First four eigen-values of the plate with $D_{x} / D_{y}=2.0 \& . H / D_{y}=0.3$

14 Summary of maximum vertical displacement at the center of the T-beam bridge deck under a single moving load 
15 Summary of maximum vertical displacement at the center of the T-beam bridge deck under two moving loads

16 Comparison of the first four natural frequencies of the isotropic plate from eigenvalue analysis in NISA with the eigen-values calculated in MATLAB

17 Comparison of maximum vertical displacements with the displacements investigated by Jong-Shyong Wu. Et. al. [15]

18 Summary of maximum vertical displacement at the center of the T-beam bridge deck under a single moving load in NISA

19 Summary of maximum vertical displacement at the center of the T-beam bridge deck under two moving loads in NISA

20 Comparison of suspension forces at different speeds for HS20-44 truck on a Tbeam bridge deck

21 Comparison of tire forces at different speeds for HS20-44 truck on a T-beam bridge deck.

22 Total axle load of an AASHTO HS20-44 truck on a T-beam bridge deck

23 Maximum vertical displacements at the center of the T-beam bridge deck under an AASHTO HS20-44 truck moving with different speeds by the orthotropic plate theory

24 Maximum vertical displacements at the center of the T-beam bridge deck under an AASHTO HS20-44 truck moving with different speeds by FEM

25 Comparison of maximum vertical displacements at the center of the T-beam bridge deck under an AASHTO HS20-44 truck moving with different speeds by the orthotropic plate theory and by the finite element analysis method 


\section{ACKNOWLEDGEMENTS}

It is with great pleasure that I offer the most heartfelt thanks to my advisor, Dr. Sukhvarsh Jerath, for his unconditional and infinite support throughout my Ph. D. study. I will always treasure the wisdom and experiences he shared with me in the field of engineering and in daily life. I also wish to express my gratitude to Dr. William Semke, Dr. George Bibel, Dr. Iraj Mamaghani, and Dr. Michael Mann for their continued support by being on the committee along with their valuable suggestions and timely help rendered throughout the period of this study.

I am grateful to Dr. Charles Moretti, Department Chair of Civil Engineering, for providing me with the opportunity to work as a Graduate Teaching Assistant during my $\mathrm{Ph}$. D. program at the School of Engineering and Mines. The experience of teaching was incredibly rewarding. I am also thankful to Mary Jo Rasmussen for her tremendous help at all times.

Last but not least I want to thank my family who, although thousands of miles away, live very close to my heart. Their continued encouragement, silent concern and endless love converge to my momentum to work hard and achieve the best I can. I am also greatly indebted to my friends without whom I do not think I could have overcome the challenges of being in a different culture and a new community. 
To Aai and Aaba 


\begin{abstract}
Moving loads have great effect on dynamic stresses in structures and cause them to vibrate extensively, especially at high velocities. A peculiar feature of moving loads is that they are variable in both space and time. This is why the dynamic analysis of bridges under moving forces has attracted researchers worldwide. When a moving load is traveling on a bridge, different factors play an important role in the vibration of the bridge. Road surface profile, vehicle dynamics, weight and speed of the moving vehicle and the geometry of the bridge all play an important roles in the analysis. The main objective of this research work is to study the collective effect of all these factors over the impact factor.

Road surface roughness is generated by using a Power Spectral Density function which represents different classes of roads. A 12 Degree Of Freedom model of an HS2044 truck is modeled and an interactive function of this model with the road surface roughness is developed to find an increased load which is applied on the bridge decks to find the dynamic response. The bridge deck is analyzed by using analytical and numerical methods. An orthotropic plate theory is used to solve the bridge deck analytically and the finite element analysis method is used to solve the bridge numerically. The increased load calculated from the interaction function of road surface roughness and vehicle model is simulated as a train of moving loads by using Dirac-delta function in the orthotropic plate theory. The same train of moving loads is simulated in finite element analysis by using
\end{abstract}


arrival time and time function data for the nodal points along the moving path of the truck load.

Dynamic response is calculated in terms of the vertical deflection at the center of the bridge deck and compared with the static deflection where the load is considered to be steady. For the bridge deck under investigation, the impact factor given by AASHTO underestimates the dynamic effect under the moving loads. This might be because of the inability of the impact factor formula given by AASHTO, which is a function of span length of the bridge deck, to take into account the effect of road surface roughness, vehicle dynamics, vehicle weight, and vehicle speeds. Its is suggested that it is necessary to do the detailed dynamic analysis of bridges by considering road surface roughess, vehicle dynamics, vehicle weight, and vehicle speed. 


\section{CHAPTER I}

\section{INTRODUCTION}

\section{I-a: General Description}

Vibration of bridges due to moving vehicles is important for two reasons. First, the stresses are increased above those due to static-load case, which is normally accounted by the "impact factor" in design. The second reason is that excessive vibration may have the psychological effect of impairing public confidence in the structure. The impact factor is provided in the "Standard Specifications for Highway Bridges" by AASHTO [1] as,

$$
I=\frac{50}{L+125}
$$

Where,

$\mathrm{I}=$ Impact Factor (maximum 30 percent)

$\mathrm{L}=$ Length in feet of the portion of the span that is loaded to produce the maximum stress in the member

If we see this impact factor, it is a function of only the span length of the bridge, where in reality impact factor is affected by many other variables. Moving loads have great effect on dynamic stresses in structures and cause them to vibrate extensively, especially at high velocities. Peculiar feature of moving loads is that they are variable in both the space and time. As the moving load travels on bridge with high velocities, it imparts vibrations to the bridge which in turn increases stresses in the members above their values under static loading. When a moving load travels on a multilane bridge, 
different factors play important role in deciding the dynamic impact. First of all, the vehicles have their own dynamic system with suspension and damping, and when these vehicles move on bridge decks with different road surface profiles, they impart more load than the static load values on bridge decks. Bridge characteristics such as its natural frequencies and damping also play a very important role in deciding the dynamic impact. Other important factors in analysis are weight and speed of the vehicle, and location of the vehicle on a bridge deck. It is therefore necessary to do the detailed dynamic analysis of bridges under moving loads to predict increase in the stresses.

\section{I-b: Objectives}

The behavior of highway bridges under moving loads is a subject of investigation with many researchers from early 1950s. Some of the researchers have studied a role of road surface roughness on the increased load and some have studied a bridge deck behavior under a constant load or a train of loads moving across the bridge deck with different speeds. C. J. Dodds and J. D. Robson [3] have studied the road surface roughness and the free vibrations of rectangular orthotropic plates with parallel edges simply supported are studied by Jayaraman G. et. al. [6]. Very few researchers have studied the forced vibrations of the orthotropic plates under moving loads. Bridge decks can be considered as orthotropic plates on which moving loads can be simulated by using Dirac-delta function. The main objective of this research work is to combine the effects of the road surface roughness, vehicle dynamics to get the increased load on the bridge deck and simulate this increased load on the orthotropic plates by using Dirac-delta function and do the forced vibrations analysis of orthotropic plates under the increased 
load at different speeds and compare the results with the finite element analysis of the same bridge.

The general objective of this research is to

1: Generate different road surface roughness profiles.

2: $\quad$ Develop a 12 DOF model of an AASHTO HS20-44 truck.

3: Study the effect of AASHTO HS20-44 truck on a bridge deck by considering it as an orthotropic plate with two opposite edges simply supported and the other two edges free, and simulate moving loads by using a Dirac-Delta function to investigate the dynamic effect.

4: $\quad$ Model the same bridge deck in a finite element analysis program and simulate moving loads on it to study the dynamic effect.

5: Compare results from the analysis of an orthotropic plate and the finite element analysis.

The first objective is achieved in Chapter III where different road surface roughness profiles are generated by using a power spectral density function. Two different classes of road surface profiles are considered for the study. A 12 DOF model of an HS20-44 truck is developed in Chapter IV. The interaction of this truck with a road surface profile is derived to find the increased load effect of the truck which will give us the effect of different road surface profiles and vehicle dynamics. The truck, which has the increased load effect from the truck dynamics and road surface roughness calculated in Chapter IV, is used as a moving load vehicle on the bridge decks to investigate its dynamic effect. In Chapter V, a simply supported bridge deck is modeled as an orthotropic plate with two opposite edges simply supported and two edges free. The 
natural frequencies of this plate depend upon the flexural and torsional rigidities of the plate. Orthotropic plates are divided into two categories depending on their natural frequencies and rigidities, and different frequency equations for different categories of the plates are derived and moving loads are simulated on the plate to get an equation of motion. Equation of motion is then solved by using fourth order Runge-Kutta method to get the analytical results. The same bridge deck is then modeled in great details by using the finite element analysis package 'NISA' [2] in Chapter VI. Moving loads are simulated in NISA [2] by the use of arrival time and time function data. 


\section{CHAPTER II}

\section{LITERATURE REVIEW}

C. J. Dodds and J. D. Robson [3] studied the road surface roughness and showed that the typical road surface may be considered as realization of homogeneous and isotropic Gaussian random process and proposed a new road classification method based on power spectral density function. J. G. S. da Silva [4] presented an analysis methodology to evaluate the dynamic effect on highway bridge decks due to vehicles crossing on the rough surface defined by a probabilistic model. He obtained the results mainly for heavy vehicles moving over rough reinforced concrete highway bridge deck made of a straight box section girder. It was verified in all the cases studied in this investigation, for usual vehicle velocities, that the dynamic effect on highway bridge decks due to the interaction of the vehicle suspension flexibility with a smooth pavement surface can be as high as $90 \%$. T. L. Wang et. al. [5] studied dynamic response of highway trucks due to road surface roughness and used the road classification method proposed by C. J. Dodds and J. D. Robson [3] and generated different types of road surface profiles. They developed vehicle models for H20-44 and HS20-44 trucks with seven or twelve degrees of freedom respectively and obtained impact factors for the suspension and tire forces for vehicle model running on different classes of roads at various speeds. 
Free vibrations of rectangular orthotropic plates with parallel edges simply supported are studied by Jayaraman G. et. al. [6]. They studied the influence of the material orthotrophy, foundation modulus and aspect ratio on the natural frequencies. They also studied the influence of different boundary condition on the natural frequencies. According to Jayaraman G. et. al. [6] the influence of the ratio of the flexural rigidities in $\mathrm{x}$ and $\mathrm{y}$ directions on the natural frequencies is more for the plates with two opposite edges simply supported and two edges free; where as the influence of the ratio of torsional rigidity and flexural rigidity on the natural frequencies is more for the plates with two opposite edges simply supported and two edges clamped. X. Q. Zhu and S. S. Law [7] investigated the dynamic behavior of continuous multi-lane bridge deck from the moving vehicles. They modeled the bridge as a multi-span continuous orthotropic rectangular plate with line rigid intermediate supports and investigated the dynamic behavior of the bridge deck under single and several vehicles moving in different lanes using the orthotropic plate theory and modal superposition technique. They found that the vehicle position has an important effect on the impact factor. They also found that the impact factors associated with the multiple vehicles are smaller than those are for single vehicle.

Fryba L. [8] analytically solved the dynamic responses of uniform flat plate under a moving load and used a Dirac delta function to simulate a moving load on the plate. D. P. Thambiratnam et. al. [9] performed the experimental analysis of a reinforced concrete bridge under vehicular loads to record the strains at different locations. They investigated the dependence of the dynamic amplification of the strain on bridge deck location and 
vehicle speed, and recorded the dynamic amplification up to 1.5 , which was higher than values predicted by bridge design codes.

As we can see, in the previous studies no one has studied all the affecting factors together. The purpose of this project is to investigate the effect of road surface roughness, vehicle dynamics, bridge characteristic, weight and speed of the vehicle on the vertical deflection at different locations on the bridge decks. 


\section{CHAPTER III}

\section{ROAD SURFACE ROUGHNESS}

III-a: Power Spectral Density Function

Out of many factors affecting the impact factor of bridges, road surface roughness is the primary factor. Due to the irregularities of the road surface, moving vehicle jumps up and down, and in the process changes the static load values of the vehicle because of the suspension and damping system of the vehicle. According to the classification of roads based on road spectra presented by MIRA [10], there are three different classes of roads and every class have two, three or four road conditions as very good, good, average, and poor. In the previous studies, C. J. Dodds and J. D. Robson [3] have developed the Power Spectral Density (PSD) functions to describe the road surface roughness as,

$$
\begin{array}{ll}
S(\phi)=A\left(\frac{\phi}{\phi_{0}}\right)^{-w 1}, & \phi \leq \phi_{0} \\
S(\phi) & =A\left(\frac{\phi}{\phi_{0}}\right)^{-w 2}, \quad \phi \geq \phi_{0}
\end{array}
$$

$$
\text { Where, } \begin{aligned}
S(\phi) & =\operatorname{PSD}\left(\mathrm{m}^{2} / \text { cycle } / \mathrm{m}\right) \\
\phi & =\text { Wave number }(\text { cycle } / \mathrm{m}) \\
A & =\text { Roughness coefficient }\left(\mathrm{m}^{3} / \text { cycle }\right) \\
\phi_{0} & =\text { Discontinuity frequency }=(1 / 2 \pi)(\text { cycle } / \mathrm{m})
\end{aligned}
$$


$w 1, w 2=$ Roughness exponent.

Table 1 shows the roughness coefficient $A$ as well as exponents $w 1$ and $w 2$ as a function of road class.

Table 1: Spectrum constants according to classification of roads, MIRA [10].

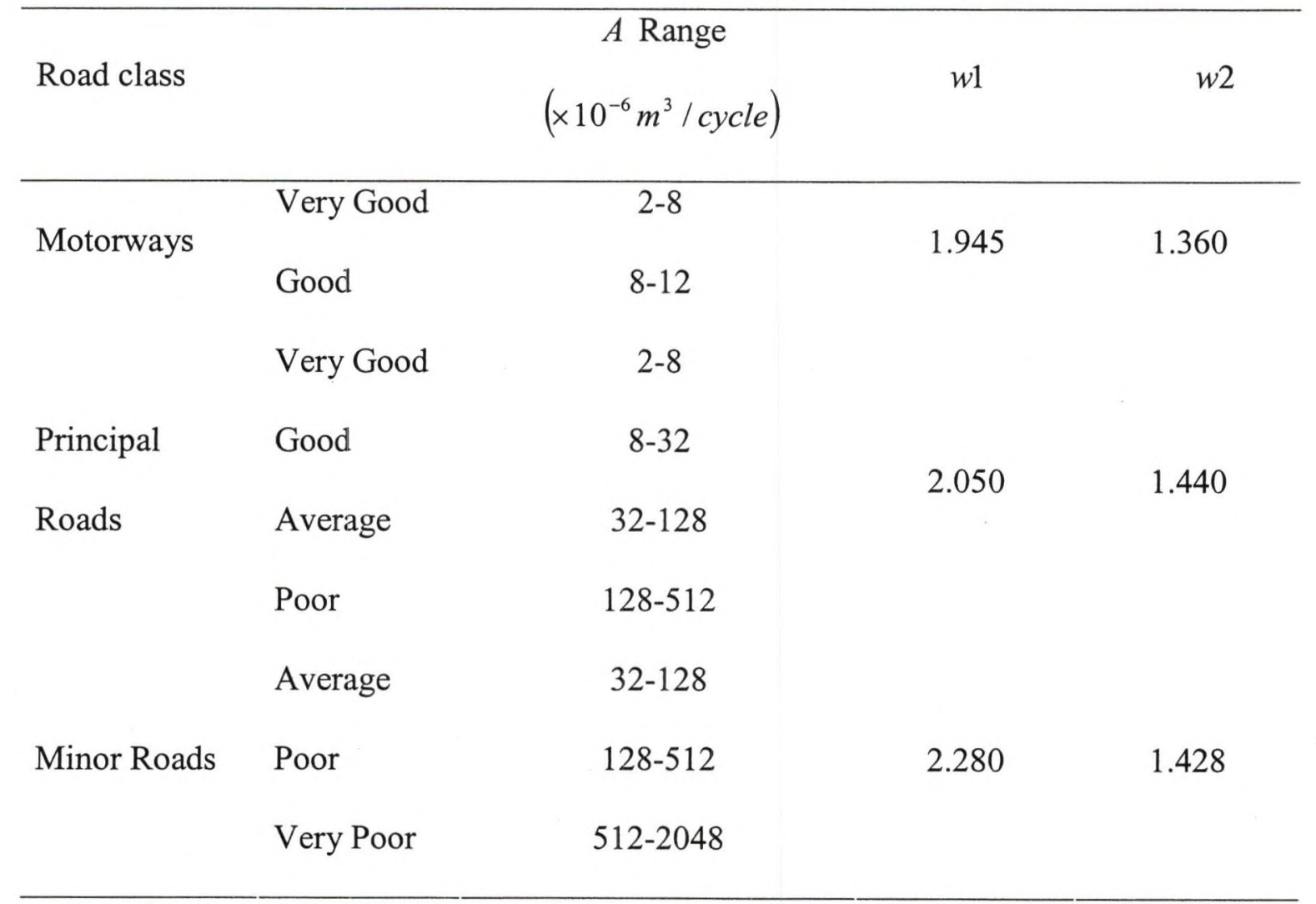

In order to simplify the description of road surface roughness, both $w 1$ and $w 2$ are assumed to have a value of 2.

The PSD function then becomes

$$
S(\phi)=A\left(\frac{\phi}{\phi_{0}}\right)^{-2}
$$

Power Spectral Density is a method of scaling the amplitude axis in certain spectra, which consists of random rather than deterministic signals. A random signal has 
energy spread out over a frequency band; it is not meaningful to calculate RMS value at any specific frequency. It only makes sense to consider its amplitude in a fixed frequency band. PSD is defined in terms of amplitude squared per frequency, and is thus proportional to the power delivered by the signal in one hertz band.

MATLAB 6.5.1 is used to generate a sequence of random numbers having Gaussian probability distribution with zero mean and approximate "white noise" properties. White noise is a signal with a flat frequency spectrum in linear space. In other words, the signal has equal power in any linear band at any center frequency having given a bandwidth. The white noise is then passed through a first order recursive filter to shape the spectrum to the form given in Equation (3.2).

\section{Generation of Sequence of Random Numbers Using MATLAB}

Digital Signal Processing (DSP Blockset) has a Random Source, which generates different types of sequence of random numbers depending upon the selection of the source type. The Gaussian (normal) distribution with Zero mean and a specified value of Variance, which depends upon the Roughness coefficient $(A)$ of Equation (3.2), is selected for the different types of Random Sources. By assigning this zero mean and the variance to the source data, a random signal is produced. The power spectral density function of this random signal is a flat line. This random signal is the input signal for the first order digital filter in MATLAB.

\section{First Order Recursive Filter in MATLAB}

The digital filter block in MATLAB independently filters each channel of the input signal with a specified digital Infinite Impulse Response (IIR) filter. Since the 
output of each input depends upon the previous inputs and outputs, these filters are called as recursive filters.

Equation of the IIR filter in MATLAB is given as

$$
y(n)=(1 / a(1)) *\left[\begin{array}{l}
b(1) * x(n)+b(2) * x(n-1)+\ldots+b(n b) * x(n-n b) \\
-a(2) * y(n-1)-\ldots a(n a+1) * y(n-n a)
\end{array}\right]
$$

If we consider only the immediately previous output sample, the recursive filter given in Equation (3.3) becomes "first order" which means, $n a=1$ which gives $n a+1=2$. To modify this filter given in Equation (3.3) to the first order filter where the input at the sample point is added to the only previous output sample. This is achieved by assigning

$$
\begin{aligned}
& a(1)=1, \\
& a(2)=-1, \\
& b(1)=1, \\
& b(2)=b(3) \ldots=b(n b)=0 .
\end{aligned}
$$

Hence the Equation (3.3) becomes

$$
y(n)=[x(n)+y(n-1)]
$$

Where,

$$
\begin{aligned}
& x(n)=\text { Input at the } n^{\text {th }} \text { sample distance } \\
& y(n)=\text { Output at the } n^{\text {th }} \text { sample distance }
\end{aligned}
$$

Equation (3.4) is identical with the first order digital filter given by Otnes and Enochson [11].

When a random signal with white noise properties is passed through this first order digital filter, the power spectral density of the output function gets modified and is related with the power spectral density of the input function through a transfer function. 
The power spectral density of the output function should match with the power spectral density function given in Equation (3.2). The variance required to generate the random signal in MATLAB is calculated by comparing this transfer function with the power spectral density function given in Equation (3.2).

The transfer function of the first order recursive filter is

$$
H(\phi)=\frac{1}{1-\exp (-n 2 \pi \lambda \phi)}
$$

Where,

$\lambda=$ Sample length / total number of samples.

By using the approximation

$$
\begin{aligned}
& \exp (x)=1+x \\
& H(\phi)=\frac{1}{1-(1+(-n 2 \pi \lambda \phi))}
\end{aligned}
$$

$$
\text { i.e. } H(\phi)=\frac{1}{n 2 \pi \lambda \phi}
$$

The absolute value of the squared of the transfer function is

$$
|H(\phi)|^{2}=\left(\frac{1}{2 \pi \lambda \phi}\right)^{2}=\left(\frac{\phi_{0}}{\lambda \phi}\right)^{2}=\frac{1}{\lambda^{2}}\left(\frac{\phi}{\phi_{0}}\right)^{-2}
$$

The output PSD of this filter, in response to a continuous white noise input spectrum, $N_{0}$ is,

$$
S(\phi)=|H(\phi)|^{2} S_{x}(\phi)
$$

Where, $S_{x}(\phi)=N_{0}=$ input $\mathrm{PSD}$

By substituting Equation (3.6), the above Equation becomes 


$$
S(\phi)=\frac{1}{\lambda^{2}}\left(\frac{\phi}{\phi_{0}}\right)^{-2} S_{x}(\phi)=\frac{N_{0}}{\lambda^{2}}\left(\frac{\phi}{\phi_{0}}\right)^{-2}
$$

By comparing Equation (3.2) and (3.7), we get

$$
A=\frac{N_{0}}{\lambda^{2}}
$$

Which gives, $N_{0}=A \lambda^{2}$

The PSD of white noise response given by Otnes and Enochson [11] is

$$
S_{x}(\phi)=\sigma^{2} 2 \lambda
$$

Where,

$$
\sigma^{2} \text { is the variance }
$$

By comparing Equations (3.8) and (3.9), we get

$$
\begin{aligned}
& A \lambda^{2}=\sigma^{2} 2 \lambda \\
& \text { i.e. } \sigma^{2}=\frac{A \lambda}{2}
\end{aligned}
$$

Where,

$A \quad=$ Roughness coefficient from Table 1.

$\lambda \quad=$ Sample length $/$ total number of samples.

Equation (3.10) gives us the relationship between the roughness coefficient for different classes of roads and the variance used to generate a random signal in MATLAB. 


\section{III-b: Generation of Road Surface Roughness}

The following procedure was used for a numerical generation of surface roughness,

1. Generate random numbers, which have approximate white noise properties with zero mean and variance $\sigma^{2}$ (from Equation 3.10) by using Random Source of DSP Blockset using MATLAB.

2. Pass this random numbers through the first order recursive filter (IIR filter of DSP Blockset using MATLAB). The output function is the road surface roughness.

In this investigation the sampling time depends upon the length of the road and the number of samples in that length. For example, let's consider the length of the road as $256 \mathrm{~m}$ and number of samples as $2048\left(2^{11}\right)$, which gives us sampling time $(\lambda)$ as 0.125 .

If we consider a very good road surface, range of roughness coefficient according to Table 1 is $A=2$ to $8 e-6$. Let's consider $A=5 e-6$ for the simplicity.

From Equation (3.10) we get the required variance of the input random source as, $\sigma^{2}=\frac{A \lambda}{2}=\frac{5 e-06 * 0.125}{2}=3.125 e-07$

By using Mean $=$ Zero, Var $=\sigma^{2}=3.125 e-07$, and sampling time $=\lambda=0.125$ for the random source in MATLAB, we can generate random spectrum as shown in Figure 1. 


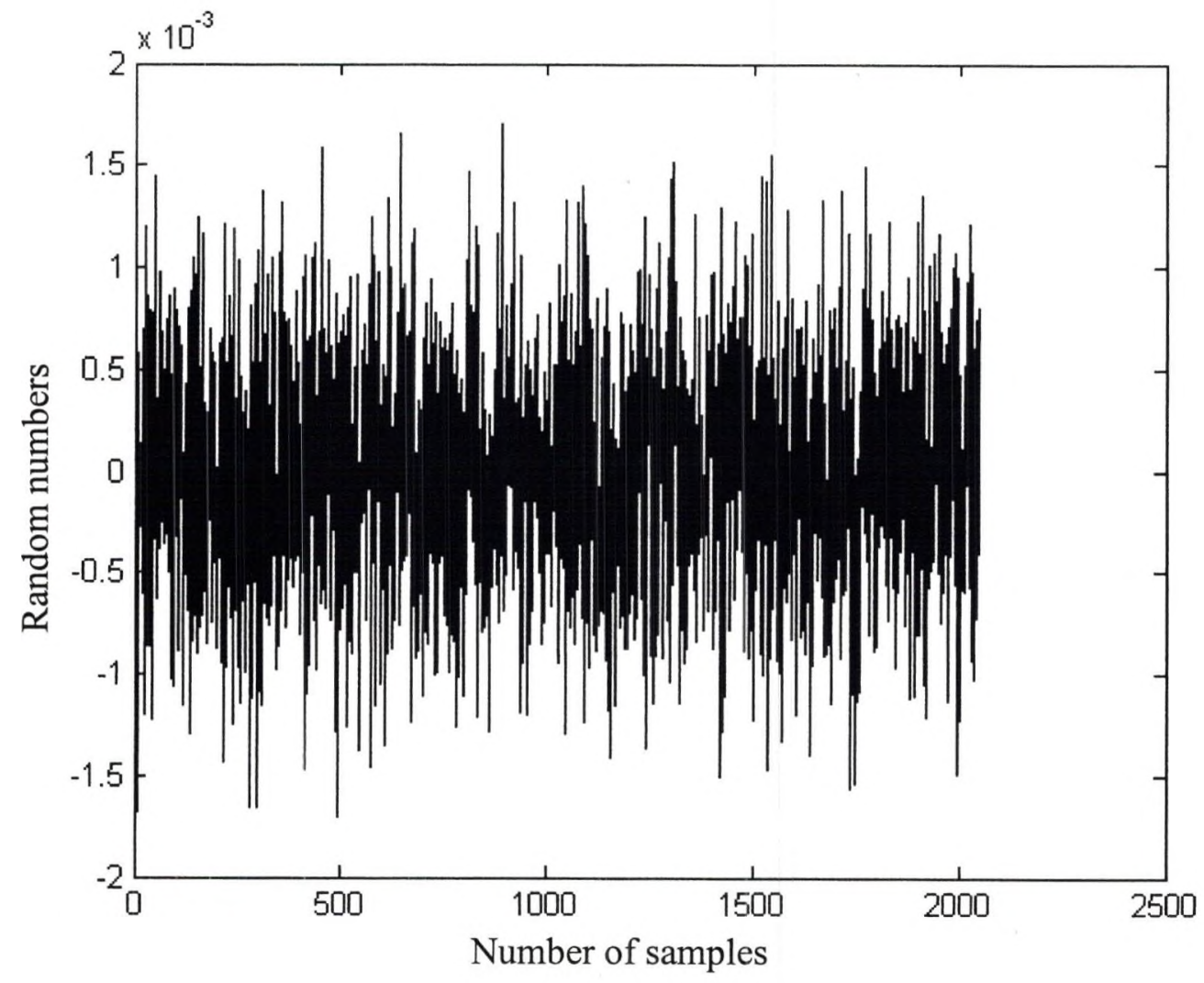

Figure 1: Spectrum of random numbers.

As we have considered the random source generator from MATLAB to have Gaussian probability distribution with approximate white noise properties, the power spectral density function of this random signal should be flat. Figure 2 shows the comparison of the power spectral densities of the random signal and that from the Equation (3.8). 


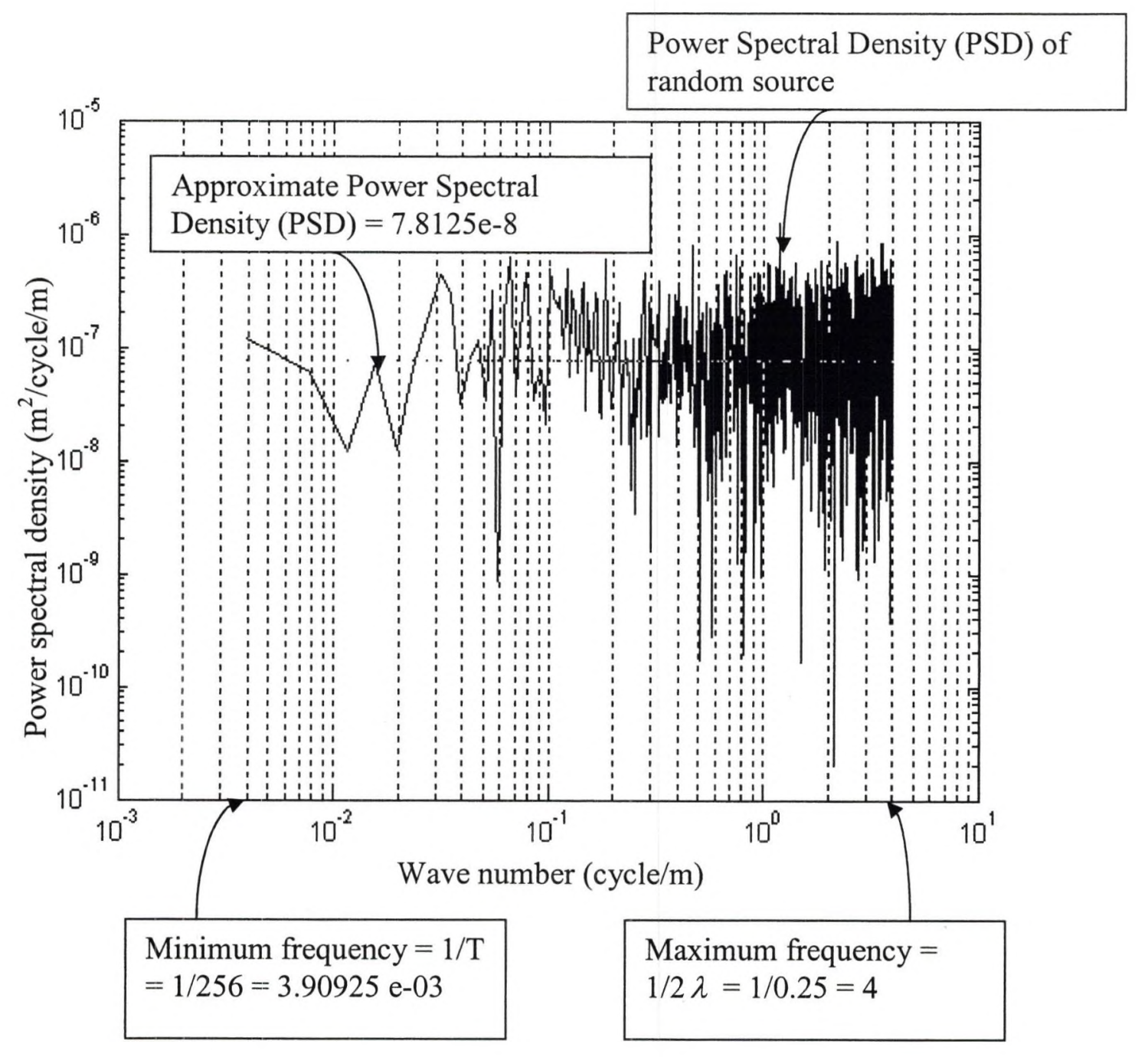

Figure 2: Comparison of Power Spectral Density of a random spectrum with the approximate Power Spectral Density.

From Figure 2, we can see that the general behavior of the PSD of the random signal is comparable with that from Equation (3.8).

When we pass this random spectrum through the first order recursive digital filter as given in Equation (3.4), output of this filter gives us the desired road surface profile as shown in Figure 3. 


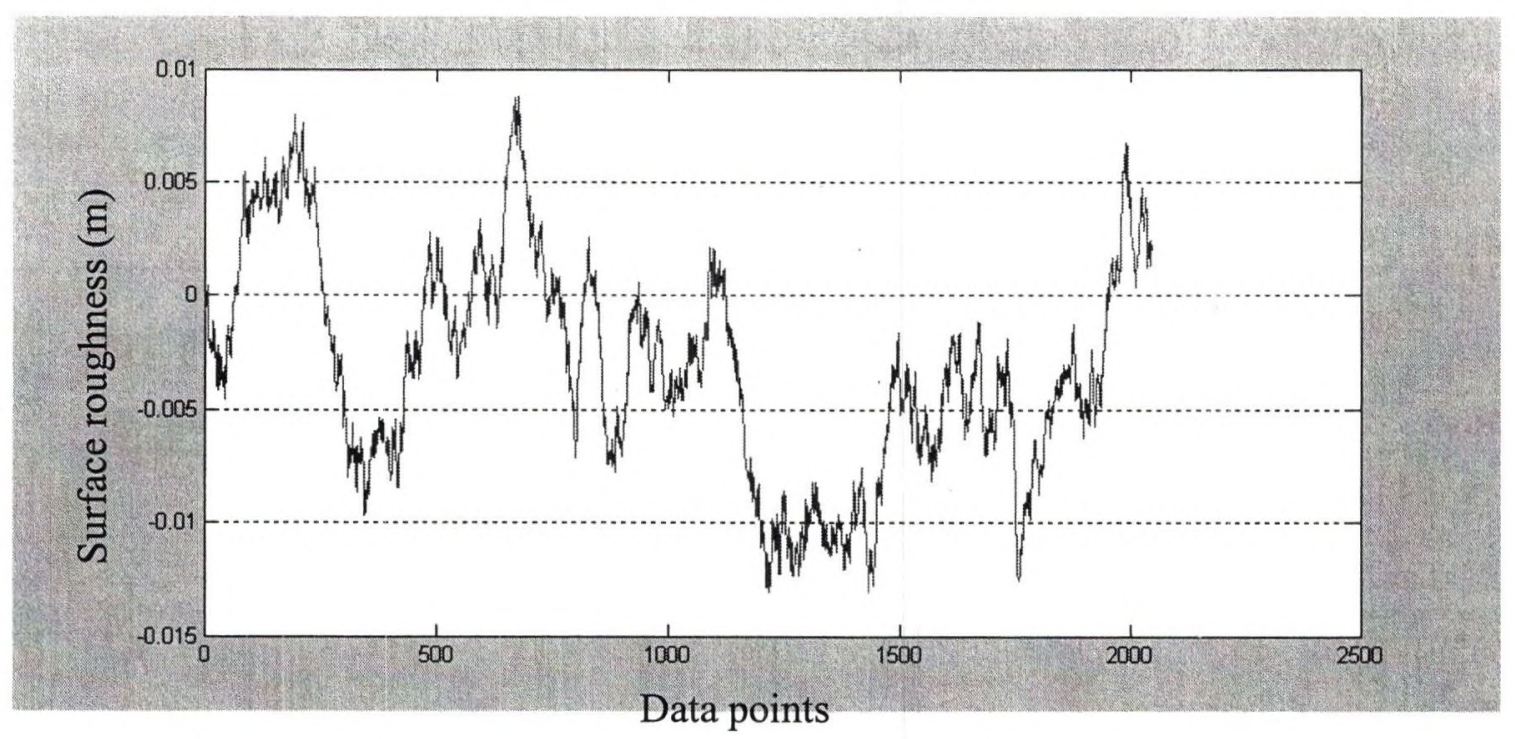

Figure 3: Output from the first order digital filter.

Figure 3 shows an example of a road surface profile generated by passing a random signal through a first order digital filter. Since we have used roughness coefficient of a very good road surface, this output signal represents a general road surface profile of a very good road surface with numbers of data points showing on $\mathrm{X}$ axis and a vertical distance between a tip of the road surface at that point from an imagined zero surface level in meters. We can see from the plot, the variation of the road surface is from $+8.5 \mathrm{~mm}$ to $-13 \mathrm{~mm}$.

The power spectral density of the output spectrum is calculated in MATLAB and compared with that given by C. J. Dodds and J. D. Robson [3]. 


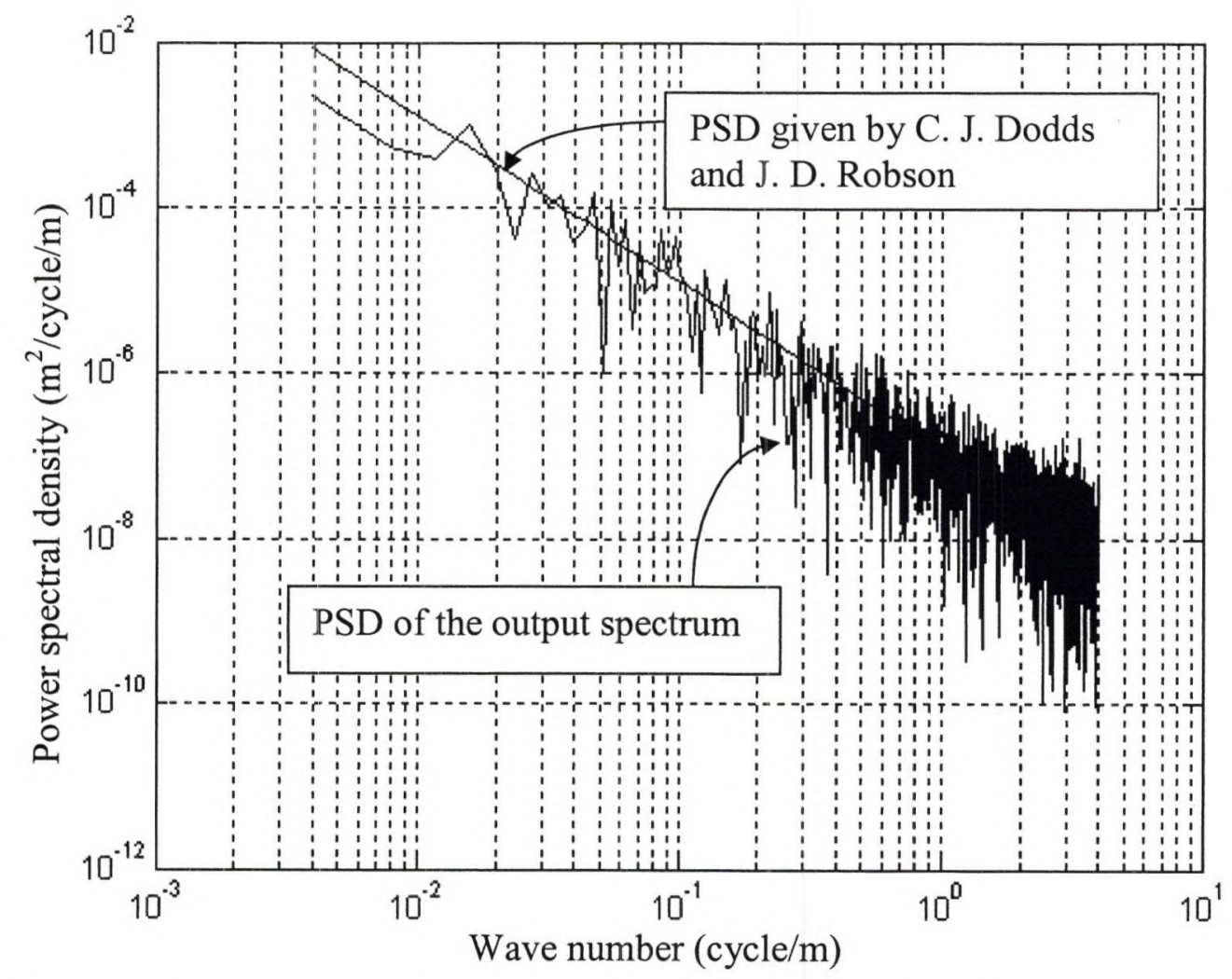

Figure 4: Comparison of PSD of the output spectrum with the PSD from C. J. Dodds et. al.

From Figure 4 we can see that, PSD calculated from the output spectrum is comparable with the PSD function proposed by C. J. Dodds and J. D. Robson [3]. Figures 5 and 6 show typical road surface profile generated by using MATLAB for a very good road surface and a good road surface. Figures 7 and 8 show the comparison of the Power Spectral Density of a very good road surface and a good road surface with the approximate Power Spectral Density respectively. 


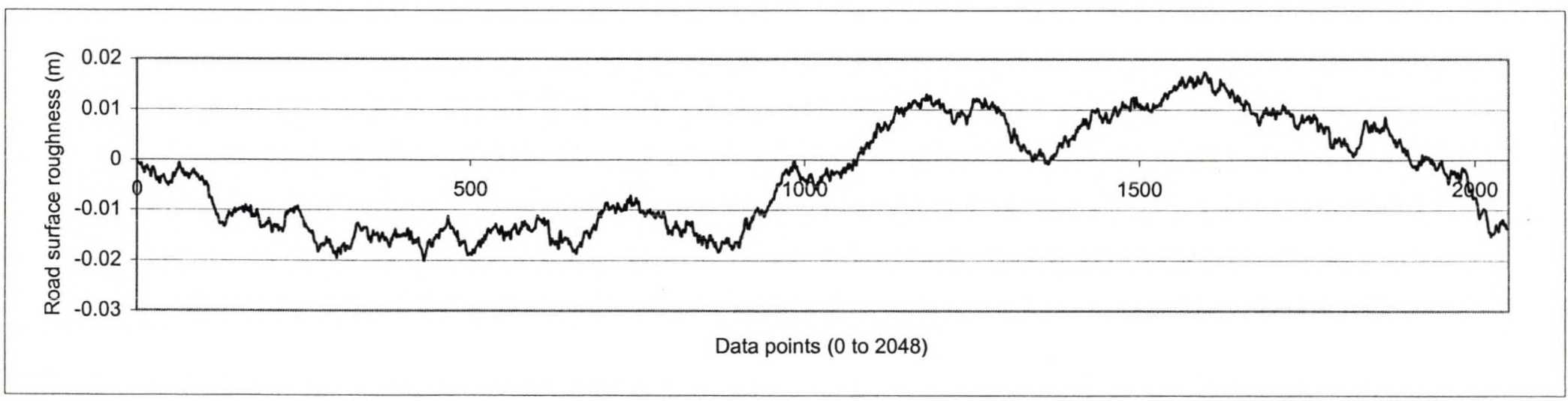

Figure 3.5: Typical road surface profile of a very good road

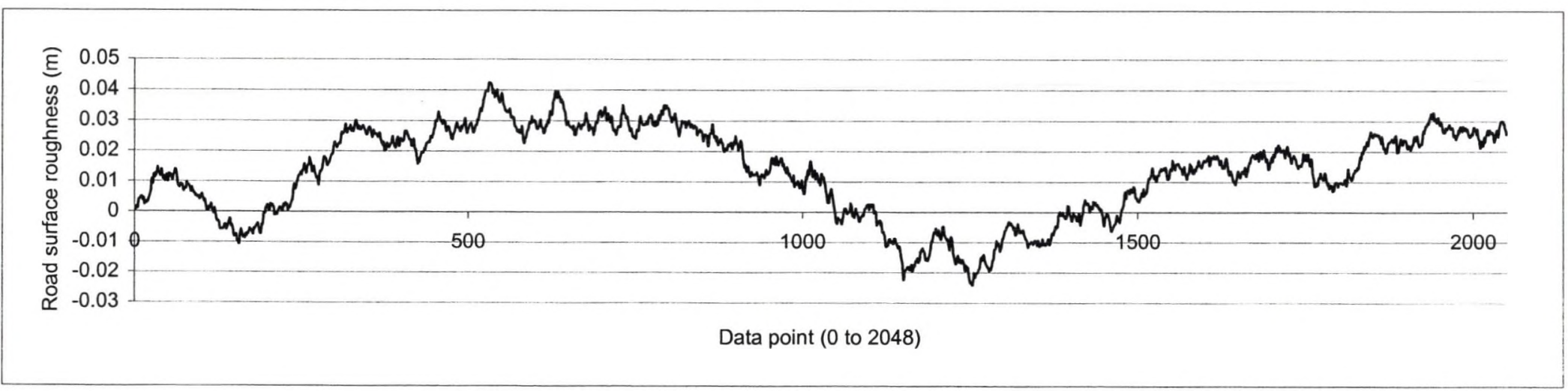

Figure 3.6: Typical road surface profile of a good road 


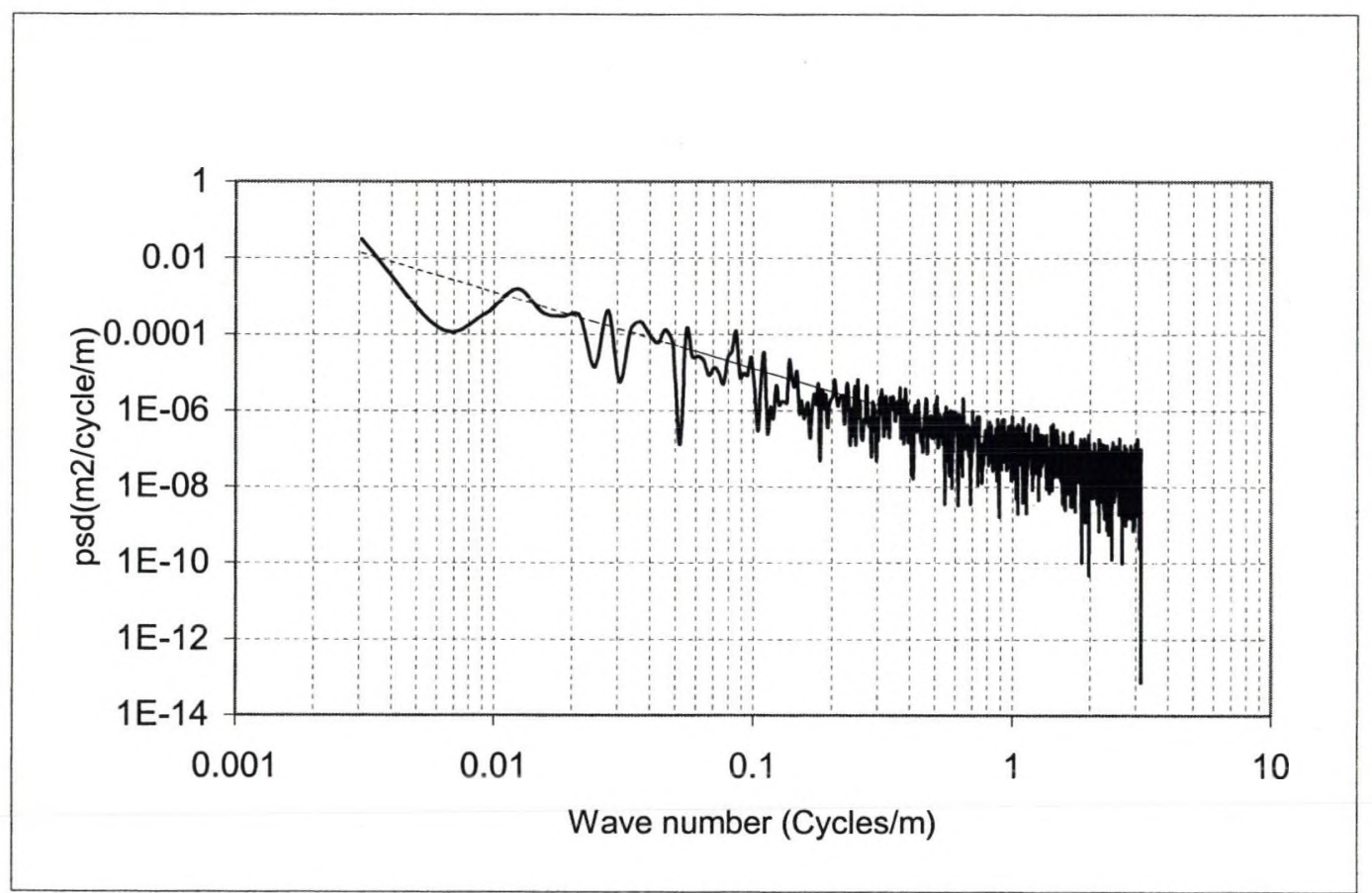

Figure 3.7: Comparison of Power Spectral Density of a very good road surface with the approximate Power Spectral Density given by C. J. Dodds and J. D. Robson [3] 


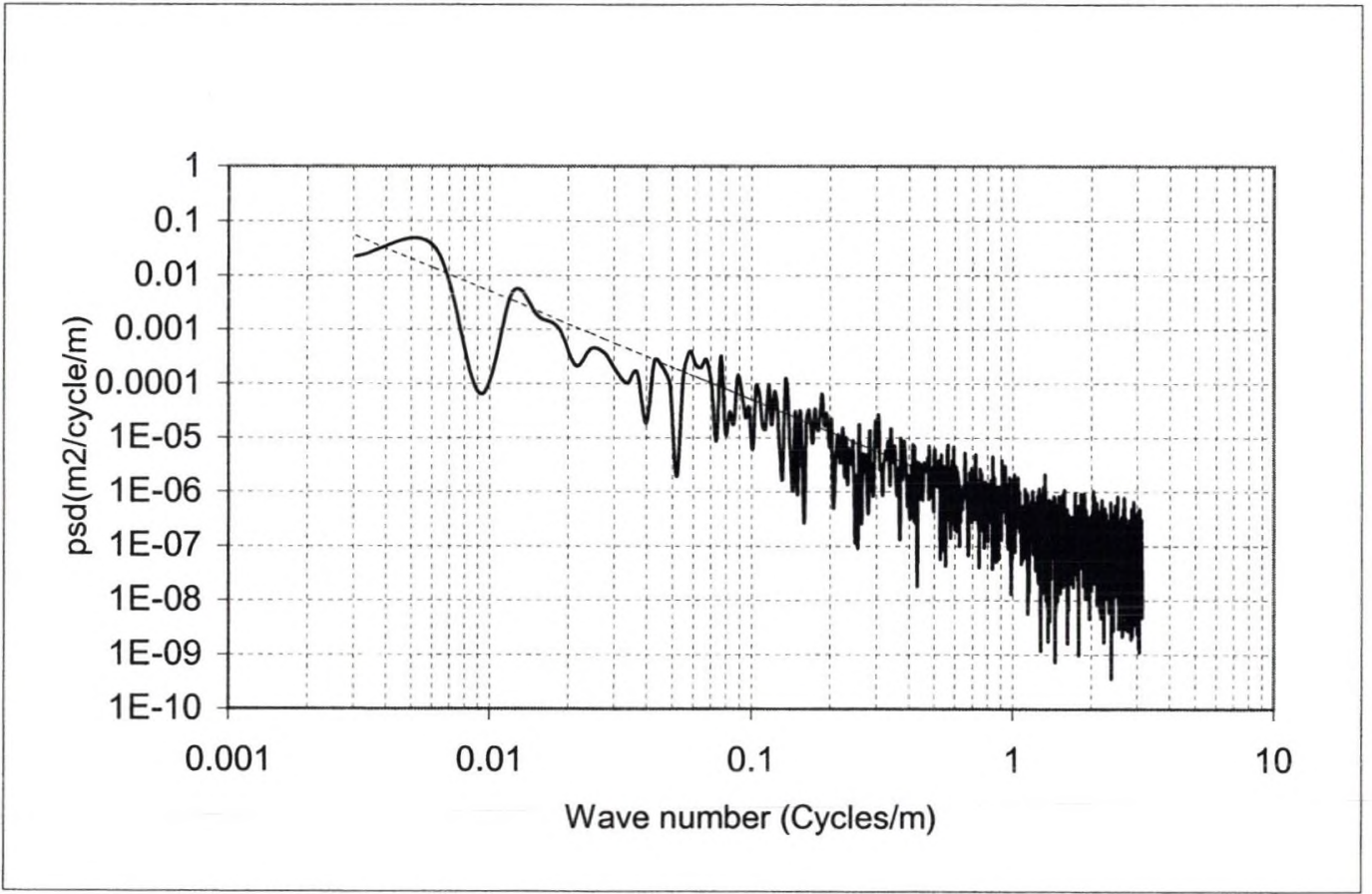

Figure 3.8: Comparison of Power Spectral Density of a good road surface with the approximate Power Spectral Density given by C. J. Dodds and J. D. Robson [3] 


\section{CHAPTER IV \\ VEHICLE DYNAMICS}

IV-a: 12 DOF Model of an AASHTO HS20-44 Truck

A nonlinear vehicle model with twelve degrees of freedom is developed according to the AASHTO HS20-44 truck [1].

Figures 9 and 10 illustrate the side and front views of the AASHTO HS20-44 [1] vehicle model. This model consists of five rigid masses as tractor, semi-trailer, steer wheel/axle set, tractor wheel/axle set, and trailer wheel/axle set. Tractor and semi-trailer are assigned three degrees of freedom $(y, \theta$, and $\phi)$ individually. Two degrees of freedom $(y$ and $\phi)$ are assigned for each wheel/axle set. The tractor and semi-trailer are interconnected at the pivot point. Truck data used for this investigation is given in Appendix- A. 


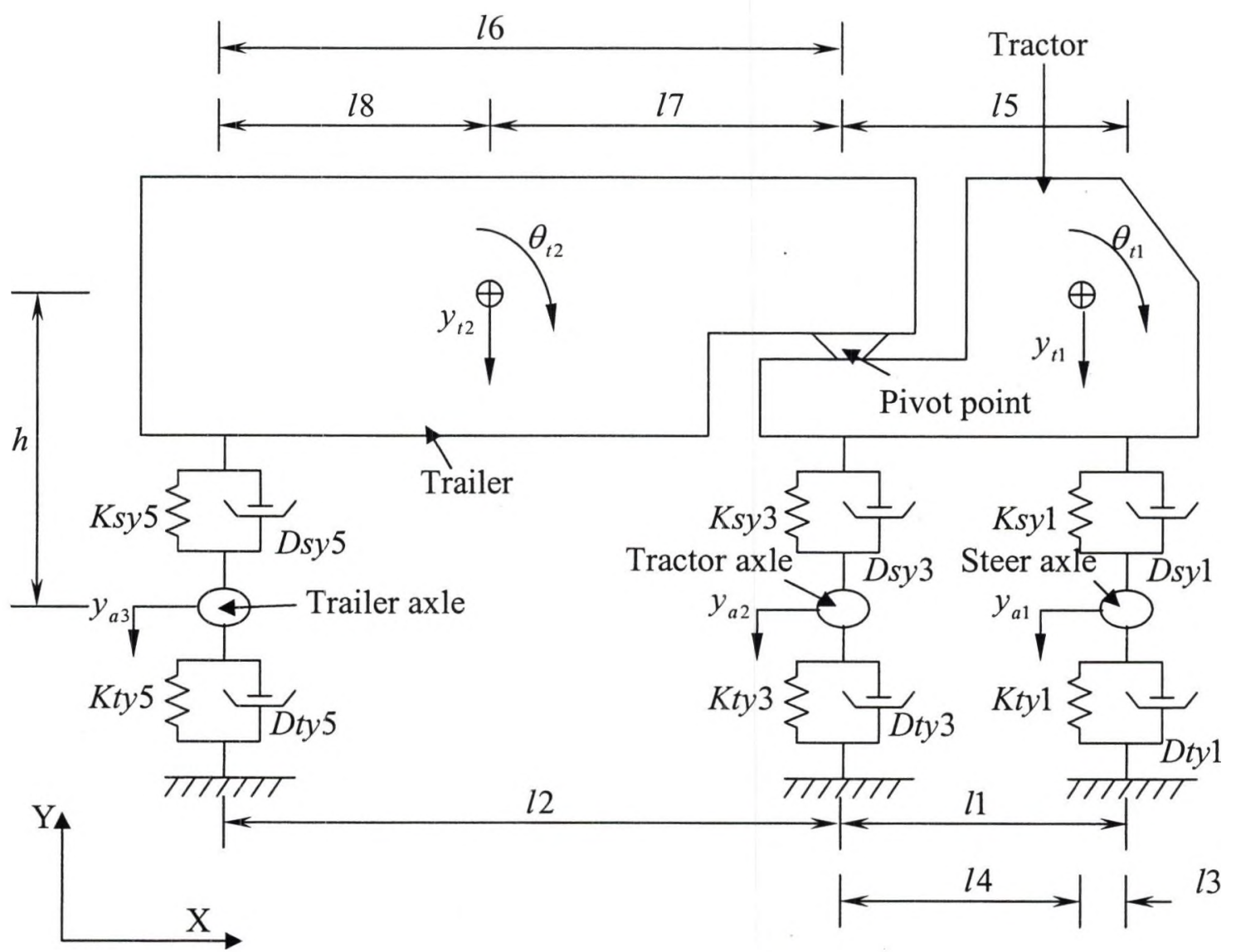

Figure 9: Side view of HS20-44 vehicle model. 


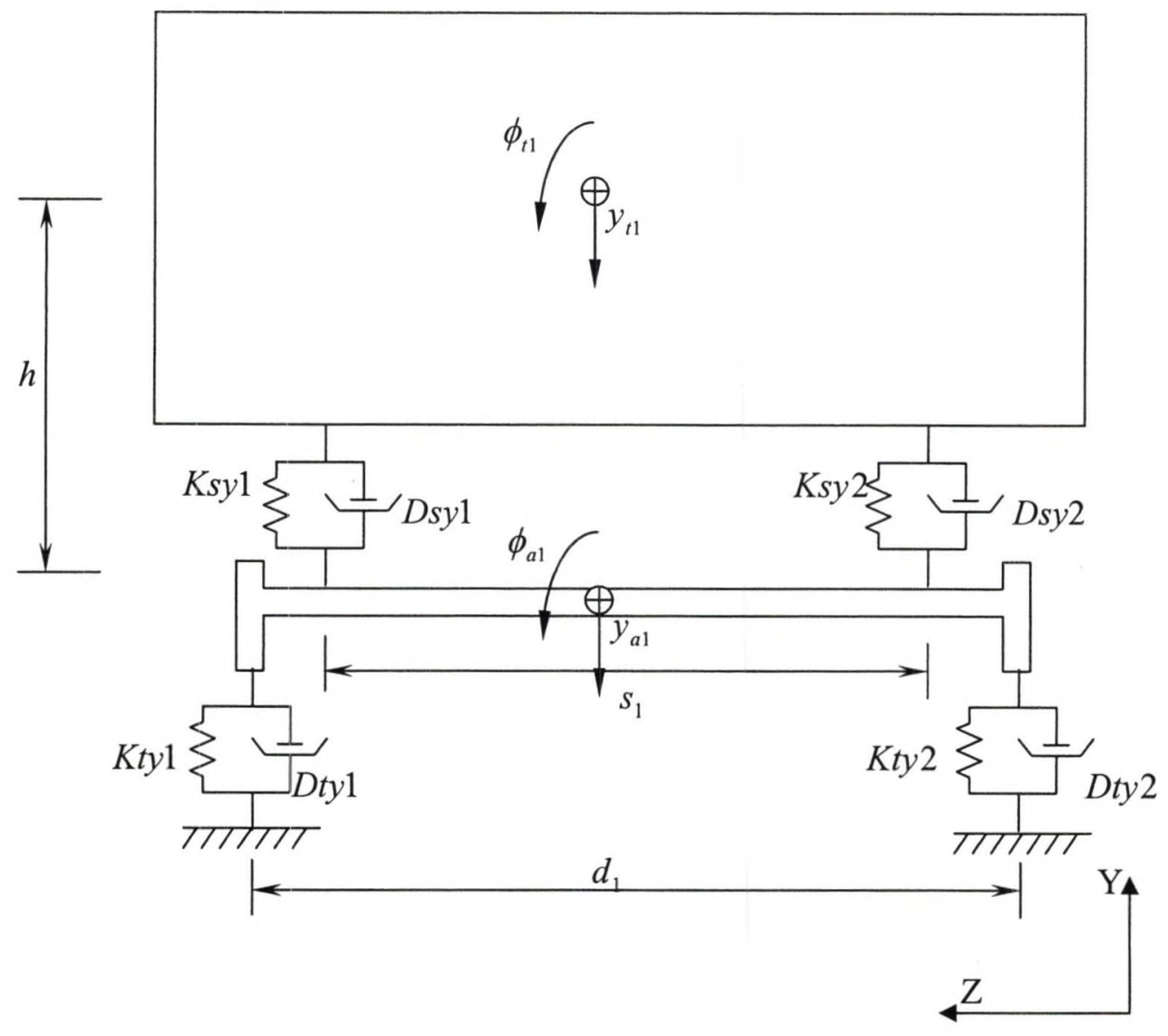

Figure 10 : Front view of HS20-44 vehicle model.

The total potential energy, $V=\sum V_{i}$ of the system is computed from the spring stiffness and relative displacements, whereas the dissipation energy, $D=\sum D_{i}$, of the system is obtained from the damping forces. Total kinetic energy, $T=\sum T_{i}$, of the system is calculated using the mass, mass moment of inertia, and translational as well as rotational velocities, of the system components.

The equations of motion of the system are derived, using Lagrange's formulation, as follows: 


$$
\frac{d}{d t}\left(\frac{\partial T}{\partial \dot{q}_{i}}\right)+\frac{\partial D}{\partial \dot{q}_{i}}-\frac{\partial T}{\partial q_{i}}+\frac{\partial V}{\partial q_{i}}=Q n c_{i}
$$

Where $q_{i}$ and $\dot{q}_{i}$ are the generalized displacements and velocities, and $Q n c_{i}$ is a generalized force.

Following are the degrees of freedom and masses of each rigid body:

$y_{t 1}, m_{t 1}=$ Tractor vertical displacement and mass

$\phi_{t 1}, I_{x t 1}=$ Tractor roll displacement and mass moment of inertia about $\mathrm{X}$ axis

$\theta_{t 1}, I_{z t 1}=$ Tractor pitch displacement and mass moment of inertia about $\mathrm{Z}$ axis

$y_{t 2}, m_{t 2}=$ Trailer vertical displacement and mass

$\phi_{t 2}, I_{x t 2}=$ Trailer roll displacement and mass moment of inertia about $\mathrm{X}$ axis

$\theta_{t 2}, I_{z t 2}=$ Trailer pitch displacement and mass moment of inertia about $\mathrm{Z}$ axis

$y_{a 1}, m_{a 1}=$ Steer axle vertical displacement and mass

$\phi_{a 1}, I_{x a 1}=$ Steer axle roll displacement and mass moment of inertia about $\mathrm{X}$ axis

$y_{a 2}, m_{a 2}=$ Tractor axle vertical displacement and mass

$\phi_{a 2}, I_{x a 2}=$ Tractor axle roll displacement and mass moment of inertia about X axis

$y_{a 3}, m_{a 3}=$ Trailer axle vertical displacement and mass

$\phi_{a 3}, I_{x a 3}=$ Trailer axle roll displacement and mass moment of inertia about $\mathrm{X}$ axis

Relative displacements at spring locations:

Suspension springs:

$$
U_{s y 1}=\left(y_{t 1}-y_{a 1}-\frac{l_{3}}{l_{5}} y_{t 2}\right)+\left(s_{1} / 2\right)\left(\phi_{t 1}-\phi_{a 1}\right)+l_{3} \theta_{t 1}-l_{7} \frac{l_{3}}{l_{5}} \theta_{t 2}
$$




$$
\begin{aligned}
& U_{s y 2}=\left(y_{t 1}-y_{a 1}-\frac{l_{3}}{l_{5}} y_{t 2}\right)-\left(s_{1} / 2\right)\left(\phi_{t 1}-\phi_{a 1}\right)+l_{3} \theta_{t 1}-l_{7} \frac{l_{3}}{l_{5}} \theta_{t 2} \\
& U_{s y 3}=\left(y_{t 1}-y_{a 2}+\frac{l_{4}}{l_{5}} y_{t 2}\right)+\left(s_{2} / 2\right)\left(\phi_{t 1}-\phi_{a 1}\right)-l_{4} \theta_{t 1}+l_{7} \frac{l_{4}}{l_{5}} \theta_{t 2} \\
& U_{s y 4}=\left(y_{t 1}-y_{a 2}+\frac{l_{4}}{l_{5}} y_{t 2}\right)-\left(s_{2} / 2\right)\left(\phi_{t 1}-\phi_{a 1}\right)-l_{4} \theta_{t 1}+l_{7} \frac{l_{4}}{l_{5}} \theta_{t 2} \\
& U_{s y 5}=\left(y_{t 2}-y_{a 3}-\frac{l_{8}}{l_{7}} y_{t 1}\right)+\left(s_{3} / 2\right)\left(\phi_{t 2}-\phi_{a 3}\right)-l_{8} \theta_{t 2}-l_{5} \frac{l_{8}}{l_{7}} \theta_{t 1} \\
& U_{s y 6}=\left(y_{t 2}-y_{a 3}-\frac{l_{8}}{l_{7}} y_{t 1}\right)-\left(s_{3} / 2\right)\left(\phi_{t 2}-\phi_{a 3}\right)-l_{8} \theta_{t 2}-l_{5} \frac{l_{8}}{l_{7}} \theta_{t 1}
\end{aligned}
$$

Tire springs:

$$
\begin{aligned}
& U_{t y 1}=y_{a 1}+\left(d_{1} / 2\right) \phi_{a 1}+\left(u_{s r 1}\right) \\
& U_{t y 2}=y_{a 1}-\left(d_{1} / 2\right) \phi_{a 1}+\left(u_{s r 2}\right) \\
& U_{t y 3}=y_{a 2}+\left(d_{2} / 2\right) \phi_{a 2}+\left(u_{s r 3}\right) \\
& U_{t y 4}=y_{a 2}-\left(d_{2} / 2\right) \phi_{a 2}+\left(u_{s r 4}\right) \\
& U_{t y 5}=y_{a 3}+\left(d_{3} / 2\right) \phi_{a 3}+\left(u_{s r 5}\right) \\
& U_{t y 6}=y_{a 3}-\left(d_{3} / 2\right) \phi_{a 3}+\left(u_{s r 6}\right)
\end{aligned}
$$

$u_{s r 1---6}=$ Vertical displacement of road surface, considered $+v e$ if upwards. 
The total kinetic energy, $T=\sum T_{i}$ of the system is

$$
\begin{gathered}
T=\frac{1}{2} m_{t 1} \dot{y}_{t 1}^{2}+\frac{1}{2} I x_{t 1} \dot{\phi}_{t 1}^{2}+\frac{1}{2} I z_{t 1} \dot{\theta}_{t 1}^{2}+\frac{1}{2} m_{t 2} \dot{y}_{t 2}^{2}+\frac{1}{2} I x_{t 2} \dot{\phi}_{t 2}^{2}+\frac{1}{2} I z_{t 2} \dot{\theta}_{t 2}^{2}+\frac{1}{2} m_{a 1} \dot{y}_{a 1}^{2}+\frac{1}{2} I x_{a 1} \dot{\phi}_{a 1}^{2} \\
+\frac{1}{2} m_{a 2} \dot{y}_{a 2}^{2}+\frac{1}{2} I x_{a 2} \dot{\phi}_{a 2}^{2}+\frac{1}{2} m_{a 3} \dot{y}_{a 3}^{2}+\frac{1}{2} I x_{a 3} \dot{\phi}_{a 3}^{2}
\end{gathered}
$$

The total potential energy, $V=\sum V_{i}$ of the system is

$$
\begin{aligned}
V=\frac{1}{2} K_{s y 1} U_{s y 1}^{2}+\frac{1}{2} K_{s y 2} U_{s y 2}^{2}+\frac{1}{2} K_{s y 3} U_{s y 3}^{2}+\frac{1}{2} K_{s y 4} U_{s y 4}^{2}+\frac{1}{2} K_{s y 5} U_{s y 5}^{2}+\frac{1}{2} K_{s y 6} U_{s y 6}^{2} \\
+\frac{1}{2} K_{t y 1} U_{t y 1}^{2}+\frac{1}{2} K_{t y 2} U_{t y 2}^{2}+\frac{1}{2} K_{t y 3} U_{t y 3}^{2}+\frac{1}{2} K_{t y 4} U_{t y 4}^{2}+\frac{1}{2} K_{t y 5} U_{t y 5}^{2}+\frac{1}{2} K_{t y 6} U_{t y 6}^{2}
\end{aligned}
$$

The dissipation energy, $D=\sum D_{i}$ of the system is

$$
\begin{aligned}
D=\frac{1}{2} D_{s y 1} \dot{U}_{s y 1}^{2} & +\frac{1}{2} D_{s y 2} \dot{U}_{s y 2}^{2}+\frac{1}{2} D_{s y 3} \dot{U}_{s y 3}^{2}+\frac{1}{2} D_{s y 4} \dot{U}_{s y 4}^{2}+\frac{1}{2} D_{s y 5} \dot{U}_{s y 5}^{2}+\frac{1}{2} D_{s y 6} \dot{U}_{s y 6}^{2} \\
& +\frac{1}{2} D_{t y 1} \dot{U}_{t y 1}^{2}+\frac{1}{2} D_{t y 2} \dot{U}_{t y 2}^{2}+\frac{1}{2} D_{t y 3} \dot{U}_{t y 3}^{2}+\frac{1}{2} D_{t y 4} \dot{U}_{t y 4}^{2}+\frac{1}{2} D_{t y 5} \dot{U}_{t y 5}^{2}+\frac{1}{2} D_{t y 6} \dot{U}_{t y 6}^{2}
\end{aligned}
$$

By substituting the relative displacements in the terms of $12 \mathrm{DOF}$ from Equations (4.2) and (4.3), the equation of motion for vertical displacement of the tractor $y_{t 1}$ can be derived as

$$
\begin{aligned}
& \frac{\partial T}{\partial \dot{y}_{t 1}}=m_{t 1} \dot{y}_{t 1} \\
& \frac{d}{d t}\left(\frac{\partial T}{\partial \dot{y}_{t 1}}\right)=m_{t 1} \ddot{y}_{t 1} \\
& \frac{\partial T}{\partial y_{t 1}}=0 \\
& \frac{\partial V}{\partial y_{t 1}}=K_{s y 1} U_{s y 1}+K_{s y 2} U_{s y 2}+K_{s y 3} U_{s y 3}+K_{s y 4} U_{s y 4}-\frac{l_{8}}{l_{7}} K_{s y 5} U_{s y 5}-\frac{l_{8}}{l_{7}} K_{s y 6} U_{s y 6}
\end{aligned}
$$


$\frac{\partial D}{\partial \dot{y}_{t 1}}=D_{s y 1} \dot{U}_{s y 1}+D_{s y 2} \dot{U}_{s y 2}+D_{s y 3} \dot{U}_{s y 3}+D_{s y 4} \dot{U}_{s y 4}-\frac{l_{8}}{l_{7}} D_{s y 5} \dot{U}_{s y 5}-\frac{l_{8}}{l_{7}} D_{s y 6} \dot{U}_{s y 6}$

And the generalized force in the vertical direction of the tractor is

$Q n c_{i}=m_{t 1} g$

By substituting Equations (4.7-4.11) into Equation (4.1), we get the equation of motion for $y_{t 1}$ as

$$
\begin{aligned}
m_{t 1} \ddot{y}_{t 1}+ & K_{s y 1} U_{s y 1}+K_{s y 2} U_{s y 2}+K_{s y 3} U_{s y 3}+K_{s y 4} U_{s y 4}-\frac{l_{8}}{l_{7}} K_{s y 5} U_{s y 5}-\frac{l_{8}}{l_{7}} K_{s y 6} U_{s y 6} \\
& +D_{s y 1} \dot{U}_{s y 1}+D_{s y 2} \dot{U}_{s y 2}+D_{s y 3} \dot{U}_{s y 3}+D_{s y 4} \dot{U}_{s y 4}-\frac{l_{8}}{l_{7}} D_{s y 5} \dot{U}_{s y 5}-\frac{l_{8}}{l_{7}} D_{s y 6} \dot{U}_{s y 6}=m_{t 1} g
\end{aligned}
$$

On the similar lines as in Equations (4.7-4.12), equations of motions are derived for the rest of the 11 DOFs and are summarized below

Equations of motion:

For Vertical Displacement of the Tractor, $y_{t 1}$ :

$$
\begin{gathered}
m_{t 1} \ddot{y}_{t 1}+\left(F_{s y 1}+F_{s y 2}+F_{s y 3}+F_{s y 4}\right)-\left(l_{8} / l_{7}\right)\left(F_{s y 5}+F_{s y 6}\right)+\left(F_{d s y 1}+F_{d s y 2}+F_{d s y 3}+F_{d s y 4}\right) \\
-\left(l_{8} / l_{7}\right)\left(F_{d s y 5}+F_{d s y 6}\right)=m_{t 1} g
\end{gathered}
$$

For Pitch Displacement of the Tractor, $\theta_{t 1}$ :

$$
\begin{aligned}
I_{z t 1} \ddot{\theta}_{t 1}+l_{3}\left(F_{s y 1}\right. & \left.+F_{s y 2}\right)-l_{4}\left(F_{s y 3}+F_{s y 4}\right)+l_{8}\left(l_{5} / l_{7}\right)\left(F_{s y 5}+F_{s y 6}\right) \\
& +l_{3}\left(F_{d s y 1}+F_{d s y 2}\right)-l_{4}\left(F_{d s y 3}+F_{d s y 4}\right)+l_{8}\left(l_{5} / l_{7}\right)\left(F_{d s y 5}+F_{d s y 6}\right)=0
\end{aligned}
$$


For Roll Displacement of the Tractor, $\phi_{t 1}$ :

$$
I_{x x 1} \ddot{\phi}_{t 1}+\left(s_{1} / 2\right)\left(F_{s y 1}-F_{s y 2}\right)+\left(s_{2} / 2\right)\left(F_{s y 3}-F_{s y 4}\right)+\left(s_{1} / 2\right)\left(F_{d s y 1}-F_{d s y 2}\right)+\left(s_{2} / 2\right)\left(F_{d s y 3}-F_{d s y 4}\right)=0
$$

For Vertical Displacement of the Trailer, $y_{t 2}$ :

$$
\begin{aligned}
m_{t 2} \ddot{y}_{t 2} & -\left(l_{3} / l_{5}\right)\left(F_{s y 1}+F_{s y 2}\right)+\left(l_{4} / l_{5}\right)\left(F_{s y 3}+F_{s y 4}\right)+\left(F_{s y 5}+F_{s y 6}\right) \\
& -\left(l_{3} / l_{5}\right)\left(F_{d s y 1}+F_{d s y 2}\right)+\left(l_{4} / l_{5}\right)\left(F_{d s y 3}+F_{d s y 4}\right)+\left(F_{d s y 5}+F_{d s y 6}\right)=m_{t 2} g
\end{aligned}
$$

For Pitch Displacement of the Trailer, $\theta_{t 2}$ :

$$
\begin{aligned}
I_{z t 2} \ddot{\theta}_{t 2} & -l_{3}\left(l_{7} / l_{5}\right)\left(F_{s y 1}+F_{s y 2}\right)+l_{4}\left(l_{7} / l_{5}\right)\left(F_{s y 3}+F_{s y 4}\right)-l_{8}\left(F_{s y 5}+F_{s y 6}\right) \\
& -l_{3}\left(l_{7} / l_{5}\right)\left(F_{d s y 1}+F_{d s y 2}\right)+l_{4}\left(l_{7} / l_{5}\right)\left(F_{d s y 3}+F_{d s y 4}\right)-l_{8}\left(F_{d s y 5}+F_{d s y 6}\right)=0
\end{aligned}
$$

For Roll Displacement of the Trailer, $\phi_{t 2}$ :

$$
I_{x t 2} \ddot{\phi}_{t 2}+\left(s_{3} / 2\right)\left(F_{s y 5}-F_{s y 6}\right)+\left(s_{3} / 2\right)\left(F_{d s y 5}-F_{d s y 6}\right)=0
$$

For Vertical Displacement of the Steer axle, $y_{a 1}$ :

$$
m_{a 1} \ddot{y}_{a 1}-\left(F_{s y 1}+F_{s y 2}\right)+\left(F_{t y 1}+F_{t y 2}\right)-\left(F_{d s y 1}+F_{d s y 2}\right)+\left(F_{d t y 1}+F_{d t y 2}\right)=m_{a 1} g
$$

For Roll Displacement of the Steer axle, $\phi_{a 1}$ :

$$
I_{x a 1} \ddot{\phi}_{a 1}-\left(s_{1} / 2\right)\left(F_{s y 1}-F_{s y 2}\right)+\left(d_{1} / 2\right)\left(F_{t y 1}-F_{t y 2}\right)-\left(s_{1} / 2\right)\left(F_{d s y 1}-F_{d s y 2}\right)+\left(d_{1} / 2\right)\left(F_{d t y 1}-F_{d t y 2}\right)=0
$$

For Vertical Displacement of the Steer axle, $y_{a 2}$ :

$$
m_{a 2} \ddot{y}_{a 2}-\left(F_{s y 3}+F_{s y 4}\right)+\left(F_{t y 3}+F_{t y 4}\right)-\left(F_{d s y 3}+F_{d s y 4}\right)+\left(F_{d t y 3}+F_{d t y 4}\right)=m_{a 2} g
$$


For Roll Displacement of the Steer axle, $\phi_{a 2}$ :

$$
I_{x a 2} \ddot{\phi}_{a 2}-\left(s_{2} / 2\right)\left(F_{s y 3}-F_{s y 4}\right)+\left(d_{2} / 2\right)\left(F_{t y 3}-F_{t y 4}\right)-\left(s_{2} / 2\right)\left(F_{d s y 3}-F_{d s y 4}\right)+\left(d_{2} / 2\right)\left(F_{d t y 3}-F_{d t y 4}\right)=0
$$

For Vertical Displacement of the Steer axle, $y_{a 3}$ :

$$
m_{a 3} \ddot{y}_{a 3}-\left(F_{s y 5}+F_{s y 6}\right)+\left(F_{t y 5}+F_{t y 6}\right)-\left(F_{d s y 5}+F_{d s y 6}\right)+\left(F_{d t y 5}+F_{d t y 6}\right)=m_{a 3} g
$$

For Roll Displacement of the Steer axle, $\phi_{a 3}$ :

$$
I_{x a 3} \ddot{\phi}_{a 3}-\left(s_{3} / 2\right)\left(F_{s y 5}-F_{s y 6}\right)+\left(d_{3} / 2\right)\left(F_{t y 5}-F_{t y 6}\right)-\left(s_{3} / 2\right)\left(F_{d s y 5}-F_{d s y 6}\right)+\left(d_{3} / 2\right)\left(F_{d t y 5}-F_{d t y 6}\right)=0
$$

Where,

$$
\begin{array}{ll}
F_{s y i}=K_{s y i} U_{s y i} \pm F_{y i} & \text { - Suspension spring force. } \\
F_{d s y i}=D_{s y i} \dot{U}_{s y i} & \text { - Damping force in the suspension. } \\
F_{t y i}=K_{t y i} U_{t y i} & \text { - Tire spring force. } \\
F_{d t y i}=D_{t y i} U_{t y i} & \text { - Damping force in the tire. }
\end{array}
$$

$i=1$ to 6 , and

$F_{y i}=$ The friction force at the $\mathrm{i}^{\text {th }}$ suspension.

From the equations of motion for all the 12 degrees of freedoms from Equations (4.13-4.24), we get the second order differential equation as

$$
[M]\{\ddot{q}\}+[C]\{\dot{q}\}+[K]\{q\}=\{F\}
$$

Where,

$$
[M]=\text { Global mass matrix. }
$$




$$
\begin{aligned}
& {[C]=\text { Global damping matrix. }} \\
& {[K]=\text { Global stiffness matrix. }} \\
& \{F\} \quad=\text { Global force matrix. }
\end{aligned}
$$

and,

$$
q=\left[\begin{array}{c}
y_{t 1} \\
\theta_{t 1} \\
\phi_{t 1} \\
y_{t 2} \\
\theta_{t 2} \\
\phi_{t 2} \\
y_{a 1} \\
\phi_{a 1} \\
y_{a 2} \\
\phi_{a 2} \\
y_{a 3} \\
\phi_{a 3}
\end{array}\right] \quad \text { and } \dot{q}=\left[\begin{array}{c}
\dot{y}_{t 1} \\
\dot{\theta}_{t 1} \\
\dot{\phi}_{t 1} \\
\dot{y}_{t 2} \\
\dot{\theta}_{t 2} \\
\dot{\phi}_{t 2} \\
\dot{y}_{a 1} \\
\dot{\phi}_{a 1} \\
\dot{y}_{a 2} \\
\dot{\phi}_{a 2} \\
\dot{y}_{a 3} \\
\dot{\phi}_{a 3}
\end{array}\right]
$$

Second order differential equation (4.25) can be converted in to a first order differential equation to simplify the solution.

The general form of second order differential equation is

$$
m \ddot{x}+c \dot{x}+k x=f
$$

Equation (4.26) can be written as

$$
\ddot{x}=\frac{f}{m}-\frac{c}{m} \dot{x}-\frac{k}{m} x
$$

Where, $m$ (mass), $c$ (damping coefficient), and $k$ (stiffness coefficient) are constants.

Let's assume that,

$$
x 1=x
$$

and, 


$$
x 2=\dot{x}
$$

i.e. $\quad \dot{x} 1=\dot{x}=x 2$

and

$$
\dot{x} 2=\ddot{x}=\frac{f}{m}-\frac{c}{m} \dot{x}-\frac{k}{m} x=F-\hat{c} x 2-\hat{k} \times 1
$$

We can write equations (4.27) and (4.28) in matrix form as,

$$
\left[\begin{array}{c}
\dot{x} 1 \\
\dot{x} 2
\end{array}\right]=\left[\begin{array}{cc}
0 & 1 \\
-\hat{k} & -\hat{c}
\end{array}\right]\left[\begin{array}{l}
x 1 \\
x 2
\end{array}\right]+\left[\begin{array}{l}
0 \\
1
\end{array}\right] F
$$

Which we can write as,

$$
\dot{X}=A X+B F
$$

Where,

$$
\dot{X}=\left[\begin{array}{c}
\dot{x} 1 \\
\dot{x} 2
\end{array}\right], X=\left[\begin{array}{l}
x 1 \\
x 2
\end{array}\right], A=\left[\begin{array}{cc}
0 & 1 \\
-\hat{k} & -\hat{c}
\end{array}\right] \text {, and } B=\left[\begin{array}{l}
0 \\
1
\end{array}\right]
$$

IV-b: Suspension and Tire Forces of an AASHTO HS20-44 Truck

Equation (4.30) is a first order differential equation, which is easy to solve in MATLAB. On the similar lines equation of motion (4.25) is modified to a first order differential equation and used in MATLAB to get the solutions of $q$ and $\dot{q}$.

$q$ gives us the displacements of the suspension and tire springs because of the road surface roughness. From these displacement solutions, the suspension force and tire forces are calculated as,

$$
\begin{aligned}
& F_{s y i}=K_{s y i} U_{s y i} \pm F_{y i} \\
& F_{t y i}=K_{t y i} U_{t y i}
\end{aligned}
$$

Two road surface conditions are considered in this study, a very good road surface and a good road surface. Figures 5 and 6 show the typical road surface roughness profiles 
for these two road conditions on a motorway. Suspension force and tire force histories for steer, tractor and trailer axles are shown in Figures A.1 to A.32 in Appendix-A on very good and good road surface.

The maximum suspension and tire forces on two different road surface conditions for an HS20-44 truck load with different speeds are summarized in Tables 2 to 5. From the Tables 2 and 3, for a HS20-44 truck traveling on a very good road surface, we can see that the impact suspension force is in the range of $23.47 \%$ to $44.71 \%$ of the static load for the steer axle and is reaching as much as $73.31 \%$ for the tractor axle, where as the maximum impact for the trailer axle is $51.52 \%$.

The impact for tire force is lesser than that for the suspension force. For the steer axle the impact is in the range of $21.85 \%$ to $44.21 \%$, the maximum impact for the tractor axle is $51.20 \%$ where as the maximum impact for trailer is $32.21 \%$ of the static load.

From the Tables 4 and 5, we can see that the impact values when an HS20-44 truck is traveling on a good road surface have increased noticeably those from the very good surface. The difference is much more in the steer axle than in the tractor and trailer axle.

Table 2: Comparison of suspension forces at different speeds for HS20-44 truck on a very good road surface.

\begin{tabular}{cccccccccc}
\hline $\begin{array}{c}\text { Speed } \\
\text { (mph) }\end{array}$ & $\begin{array}{c}\text { Static } \\
\text { (Kips) }\end{array}$ & $\begin{array}{c}\text { Fsy1 } \\
\text { (Kips) }\end{array}$ & \%Impact & $\begin{array}{c}\text { Static } \\
\text { (Kips) }\end{array}$ & $\begin{array}{c}\text { Fsy3 } \\
\text { (Kips) }\end{array}$ & \%lmpact & $\begin{array}{c}\text { Static } \\
\text { (Kips) }\end{array}$ & $\begin{array}{c}\text { Fsy5 } \\
\text { (Kips) }\end{array}$ & \%lmpact \\
\hline 15 & 2.9119 & 3.6661 & 25.90 & 14.1780 & 23.0926 & 62.88 & 14.5579 & 20.7822 & 42.76 \\
20 & 2.9119 & 3.5953 & 23.47 & 14.1780 & 22.0802 & 55.74 & 14.5579 & 20.1924 & 38.70 \\
25 & 2.9119 & 3.7536 & 28.91 & 14.1780 & 21.7757 & 53.59 & 14.5579 & 20.7265 & 42.37 \\
30 & 2.9119 & 3.7752 & 29.65 & 14.1780 & 24.5719 & 73.31 & 14.5579 & 22.0580 & 51.52 \\
35 & 2.9119 & 3.8733 & 33.02 & 14.1780 & 23.0898 & 62.86 & 14.5579 & 20.4900 & 40.75 \\
40 & 2.9119 & 3.8631 & 32.67 & 14.1780 & 21.5002 & 51.64 & 14.5579 & 21.3827 & 46.88 \\
45 & 2.9119 & 4.2139 & 44.71 & 14.1780 & 22.8514 & 61.18 & 14.5579 & 20.9179 & 43.69 \\
50 & 2.9119 & 4.1695 & 43.19 & 14.1780 & 23.4254 & 65.22 & 14.5579 & 20.4730 & 40.63 \\
\hline
\end{tabular}

Note: Fsy2, Fsy4, and Fsy6 are same as Fsy1, Fsy3, and Fsy5 respectively. 
Table 3: Comparison of tire forces at different speeds for HS20-44 truck on a very good road surface.

\begin{tabular}{cccccccccc}
\hline $\begin{array}{c}\text { Speed } \\
\text { (mph) }\end{array}$ & $\begin{array}{c}\text { Static } \\
\text { (Kips) }\end{array}$ & $\begin{array}{c}\text { Fty1 } \\
\text { (Kips) }\end{array}$ & \%Impact & $\begin{array}{c}\text { Static } \\
\text { (Kips) }\end{array}$ & $\begin{array}{c}\text { Fty3 } \\
\text { (Kips) }\end{array}$ & \%lmpact & $\begin{array}{c}\text { Static } \\
\text { (Kips) }\end{array}$ & $\begin{array}{c}\text { Fty5 } \\
\text { (Kips) }\end{array}$ & \%lmpact \\
\hline 15 & 3.99 & 4.902 & 22.86 & 15.97 & 23.968 & 50.08 & 15.99 & 20.37 & 27.39 \\
20 & 3.99 & 4.862 & 21.85 & 15.97 & 23.886 & 49.57 & 15.99 & 20.892 & 30.66 \\
25 & 3.99 & 5.437 & 36.27 & 15.97 & 23.423 & 46.67 & 15.99 & 20.006 & 25.12 \\
30 & 3.99 & 5.011 & 25.59 & 15.97 & 23.906 & 49.69 & 15.99 & 20.042 & 25.34 \\
35 & 3.99 & 5.252 & 31.63 & 15.97 & 22.646 & 41.80 & 15.99 & 20.202 & 26.34 \\
40 & 3.99 & 5.378 & 34.79 & 15.97 & 22.773 & 42.60 & 15.99 & 20.546 & 28.49 \\
45 & 3.99 & 5.378 & 34.79 & 15.97 & 23.54 & 47.40 & 15.99 & 21.141 & 32.21 \\
50 & 3.99 & 5.754 & 44.21 & 15.97 & 24.146 & 51.20 & 15.99 & 20.944 & 30.98 \\
\hline
\end{tabular}

Note: Fty2, Fty4, and Fty6 are same as Fty1, Fty3, and Fty5 respectively.

Table 4: Comparison of suspension forces at different speeds for HS20-44 truck on a good road surface.

\begin{tabular}{cccccccccc}
\hline $\begin{array}{c}\text { Speed } \\
\text { (Mph) }\end{array}$ & $\begin{array}{c}\text { Static } \\
\text { (Kips) }\end{array}$ & $\begin{array}{c}\text { Fsy1 } \\
\text { (Kips) }\end{array}$ & \% Impact & $\begin{array}{c}\text { Static } \\
\text { (Kips) }\end{array}$ & $\begin{array}{c}\text { Fsy3 } \\
\text { (Kips) }\end{array}$ & \% Impact & $\begin{array}{c}\text { Static } \\
\text { (Kips) }\end{array}$ & $\begin{array}{c}\text { Fsy5 } \\
\text { (Kips) }\end{array}$ & $\%$ Impact \\
\hline 15 & 2.9119 & 4.0169 & 37.95 & 14.1780 & 23.8001 & 67.87 & 14.5579 & 21.0453 & 44.56 \\
20 & 2.9119 & 4.0372 & 38.64 & 14.1780 & 22.7842 & 60.70 & 14.5579 & 21.8522 & 50.11 \\
25 & 2.9119 & 4.5662 & 56.81 & 14.1780 & 23.2621 & 64.07 & 14.5579 & 20.8640 & 43.32 \\
30 & 2.9119 & 4.3887 & 50.72 & 14.1780 & 23.5911 & 66.39 & 14.5579 & 20.8222 & 43.03 \\
35 & 2.9119 & 5.1816 & 77.95 & 14.1780 & 23.6442 & 66.77 & 14.5579 & 21.5286 & 47.88 \\
40 & 2.9119 & 5.0380 & 73.01 & 14.1780 & 24.8217 & 75.07 & 14.5579 & 22.2057 & 52.53 \\
45 & 2.9119 & 4.7742 & 63.95 & 14.1780 & 23.3828 & 64.92 & 14.5579 & 22.4409 & 54.15 \\
50 & 2.9119 & 5.5843 & 91.78 & 14.1780 & 25.8017 & 81.98 & 14.5579 & 23.1105 & 58.75 \\
\hline
\end{tabular}

Note: Fsy2, Fsy4, and Fsy6 are same as Fsy1, Fsy3, and Fsy5 respectively.

Table 5: Comparison of tire forces at different speeds for HS20-44 truck on a good road surface.

\begin{tabular}{cccccccccc}
\hline $\begin{array}{c}\text { Speed } \\
\text { (Mph) }\end{array}$ & $\begin{array}{c}\text { Static } \\
\text { (Kips) }\end{array}$ & $\begin{array}{c}\text { Fty1 } \\
\text { (Kips) }\end{array}$ & $\%$ Impact & $\begin{array}{c}\text { Static } \\
\text { (Kips) }\end{array}$ & $\begin{array}{c}\text { Fty3 } \\
\text { (Kips) }\end{array}$ & \% Impact & $\begin{array}{c}\text { Static } \\
\text { (Kips) }\end{array}$ & $\begin{array}{c}\text { Fty5 } \\
\text { (Kips) }\end{array}$ & \% Impact \\
\hline 15 & 3.99 & 5.916 & 48.27 & 15.97 & 26.675 & 67.03 & 15.99 & 20.37 & 21.59 \\
20 & 3.99 & 5.549 & 39.07 & 15.97 & 24.855 & 55.64 & 15.99 & 20.892 & 21.54 \\
25 & 3.99 & 6.3 & 57.89 & 15.97 & 24.308 & 52.21 & 15.99 & 20.006 & 22.67 \\
30 & 3.99 & 5.8442 & 46.47 & 15.97 & 23.9643 & 50.06 & 15.99 & 20.042 & 21.50 \\
35 & 3.99 & 7.1357 & 78.84 & 15.97 & 26.7785 & 67.68 & 15.99 & 20.202 & 23.01 \\
40 & 3.99 & 6.3201 & 58.40 & 15.97 & 25.3836 & 58.95 & 15.99 & 20.546 & 23.49 \\
45 & 3.99 & 6.5478 & 64.11 & 15.97 & 25.0641 & 56.94 & 15.99 & 21.141 & 24.45 \\
50 & 3.99 & 7.2386 & 81.42 & 15.97 & 25.4537 & 59.38 & 15.99 & 20.944 & 23.91 \\
\hline
\end{tabular}

Note: Fty2, Fty4, and Fty6 are same as Fty1, Fty3, and Fty5 respectively. 


\section{CHAPTER V}

\section{ANALYSIS OF AN ORTHOTROPIC PLATE UNDER MOVING LOADS}

\section{V-a: Generation of Equation of Motion}

A bridge deck can be modeled as an orthotropic rectangular plate with two opposite edges simply supported and other two edges as free. An orthogonal orthotropic plate is defined as a plate which has different elastic properties in two mutually perpendicular directions $\mathrm{x}$ and $\mathrm{y}$. Since the plate thickness is constant and the plate material is continuous, the different elastic properties in the two principal directions are due to different moduli of elasticity, $E_{x} \neq E_{y}$ and different Poisson's ratios $v_{x y} \neq v_{y x}$ of the material.

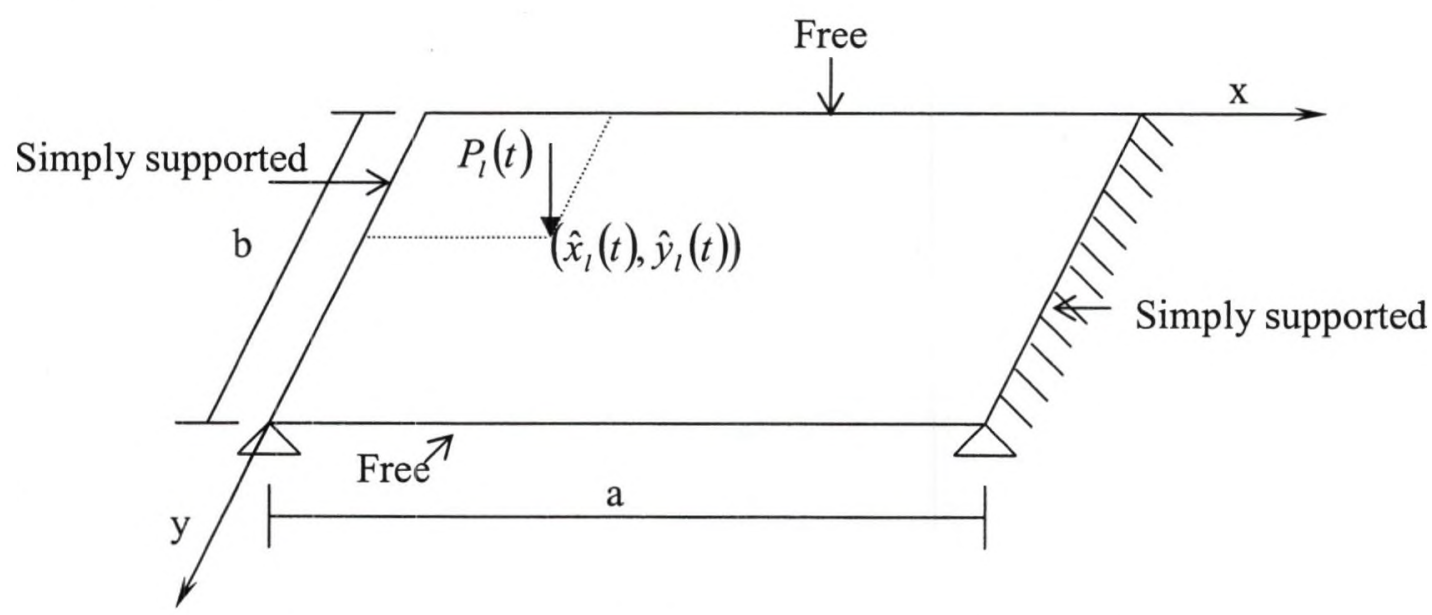

Figure 11: An orthotropic plate with a moving load 
The strain energy of an orthotropic plate is well known and is given by Timoshenko [12]

as

$$
U=\frac{1}{2} \int_{0}^{a} \int_{0}^{b}\left[D_{x}\left(\frac{\partial^{2} w}{\partial x^{2}}\right)^{2}+\left(D_{x} v_{y x}+D_{y} v_{x y}\right) \frac{\partial^{2} w}{\partial x^{2}} \frac{\partial^{2} w}{\partial y^{2}}+D_{y}\left(\frac{\partial^{2} w}{\partial y^{2}}\right)^{2}+4 D_{x y}\left(\frac{\partial^{2} w}{\partial x \partial y}\right)^{2}\right] d x d y
$$

Where,

$D_{x}=\frac{E_{x} h^{3}}{12\left(1-v_{x y} v_{y x}\right)}, D_{y}=\frac{E_{y} h^{3}}{12\left(1-v_{x y} v_{y x}\right)}$ and $D_{x y}=\frac{G_{x y} h^{3}}{12}$

$E_{x}=$ Modulus of elasticity in X direction.

$E_{y}=$ Modulus of elasticity in $\mathrm{Y}$ direction.

$v_{x y}=$ Poisson's ratio associated with a strain in the $\mathrm{Y}$ direction for a load in the $\mathrm{X}$ direction.

$v_{y x}=$ Poisson's ratio associated with a strain in the $\mathrm{X}$ direction for a load in the $\mathrm{Y}$ direction.

$G_{x y}=$ Shear modulus of the plate.

The kinetic energy of the plate is given as

$T=\frac{1}{2} \int_{0}^{a} \int_{0}^{b} \dot{w}(x, y, t)^{2} \rho h d x d y$

Where,

$\rho=$ Mass density of material of the plate.

$h=$ Thickness of the plate. 
The work done due to damping in the plate is given as

$$
W_{c}=-\int_{0}^{a} \int_{0}^{b} w(x, y, t) c_{b} \dot{w}(x, y, t) d x d y
$$

Where,

$$
c_{b}=\text { Damping coefficient of the plate. }
$$

The external work done by the force $P_{l}(t)$ can be written as

$$
W=\int_{0}^{a} \int_{0}^{b} \sum_{l=1}^{N_{p}} P_{l}(t) \delta\left(x-\hat{x}_{l}(t)\right) \delta\left(y-\hat{y}_{l}(t)\right) w(x, y, t) d x d y
$$

Where,

$$
\begin{aligned}
& P_{l}(t),\left(l=1,2, \ldots, N_{p}\right)=\text { Moving loads. } \\
& \hat{x}_{l}(t), \hat{y}_{l}(t)=\text { Coordinates of the position of moving load } P_{l}(t) . \\
& \delta\left(x-\hat{x}_{l}(t)\right), \delta\left(y-\hat{y}_{l}(t)\right)=\text { Dirac Delta function. }
\end{aligned}
$$

The displacement function of the plate can be written as a summation of the normal mode function in the $\mathrm{X}$ and $\mathrm{Y}$ coordinates as,

$$
w(x, y, t)=\sum_{m, n} w_{m n}(x, y) q_{m n}(t)
$$

Where,

$$
\begin{aligned}
& w_{m n}(x, y)=Y_{m n}(y) \sin \left(\frac{m \pi x}{a}\right)=\text { Normal modes of the plate. } \\
& q_{m n}(t)=\text { Corresponding generalized coordinates. }
\end{aligned}
$$

By using the Lagrange's equation,

$$
\frac{d}{d t}\left(\frac{\partial T}{\partial \dot{q}}\right)-\frac{\partial T}{\partial q}+\frac{\partial U}{\partial q}-\frac{\partial W_{c}}{\partial q}=\frac{\partial W}{\partial q}
$$


By substituting Equation (5.5) into Equations (5.1), (5.2), (5.3), (5.4) and by differentiating with respect to $q$ and $\dot{q}$ we get

$$
\frac{\partial U}{\partial q}=\int_{0}^{a} \int_{0}^{b}\left[D_{x}\left(\frac{\partial^{2} w_{m n}}{\partial x^{2}}\right)^{2}+\left(D_{x} v_{y x}+D_{y} v_{x y}\right) \frac{\partial^{2} w_{m n}}{\partial x^{2}} \frac{\partial^{2} w_{m n}}{\partial y^{2}}+D_{y}\left(\frac{\partial^{2} w_{m n}}{\partial y^{2}}\right)^{2}+4 D_{x y}\left(\frac{\partial^{2} w_{m n}}{\partial x \partial y}\right)^{2}\right] q_{m n}(t) d x d y
$$

Equation (5.2) becomes

$$
\begin{aligned}
& T=\frac{1}{2} \int_{0}^{a} \int_{0}^{b} w_{m n}^{2}(x, y) \dot{q}^{2}{ }_{m n}(t) \rho h d x d y \\
& \frac{\partial T}{\partial q}=0 \\
& \frac{\partial T}{\partial \dot{q}}=\int_{0}^{a} \int_{0}^{b} w_{m n}^{2}(x, y) \dot{q}_{m n}(t) \rho h d x d y \\
& \frac{d}{d t}\left(\frac{\partial T}{\partial \dot{q}}\right)=\int_{0}^{a} \int_{0}^{b} w_{m n}^{2}(x, y) \ddot{q}_{m n}(t) \rho h d x d y
\end{aligned}
$$

Equation (5.3) becomes

$$
\begin{aligned}
& W_{c}=-\int_{0}^{a} \int_{0}^{b} w_{m n}(x, y) q_{m n}(t) c_{b} w_{m n}(x, y) \dot{q}_{m n}(t) d x d y \\
& \frac{\partial W_{c}}{\partial q}=-\int_{0}^{a} \int_{0}^{b} w_{m n}(x, y) c_{b} w_{m n}(x, y) \dot{q}_{m n}(t) d x d y
\end{aligned}
$$

Equation (5.4) becomes

$$
\begin{aligned}
& W=\int_{0}^{a} \int_{0}^{b} \sum_{l=1}^{N_{p}} P_{l}(t) \delta\left(x-\hat{x}_{l}(t)\right) \delta\left(y-\hat{y}_{l}(t)\right) w_{m n}(x, y) q_{m n}(t) d x d y \\
& \frac{\partial W}{\partial q}=\int_{0}^{a} \int_{0}^{b} \sum_{l=1}^{N_{p}} P_{l}(t) \delta\left(x-\hat{x}_{l}(t)\right) \delta\left(y-\hat{y}_{l}(t)\right) w_{m n}(x, y) d x d y
\end{aligned}
$$


By substituting Equations (5.7), (5.8), (5.9), (5.10), (5.11) into Equation (5.6), we get

$$
\begin{aligned}
& \int_{0}^{a} \int_{0}^{b} w^{2}{ }_{m n}(x, y) \ddot{q}_{m n}(t) \rho h d x d y \\
& + \\
& \int_{0}^{a} \int_{0}^{b}\left[D_{x}\left(\frac{\partial^{2} w_{m n}}{\partial x^{2}}\right)^{2}+\left(D_{x} v_{y x}+D_{y} v_{x y}\right) \frac{\partial^{2} w_{m n}}{\partial x^{2}} \frac{\partial^{2} w_{m n}}{\partial y^{2}}+D_{y}\left(\frac{\partial^{2} w_{m n}}{\partial y^{2}}\right)^{2}+4 D_{x y}\left(\frac{\partial^{2} w_{m n}}{\partial x \partial y}\right)^{2}\right] q_{m n}(t) d x d y \\
& + \\
& \int_{0}^{a} \int_{0}^{b} w_{m n}(x, y) c_{b} w_{m n}(x, y) \dot{q}_{m n}(t) d x d y=\int_{0}^{a} \int_{0}^{b} \sum_{l=1}^{N_{p}} P_{l}(t) \delta\left(x-\hat{x}_{l}(t)\right) \delta\left(y-\hat{y}_{l}(t)\right) w_{m n}(x, y) d x d y
\end{aligned}
$$

This can be written as

$$
M \ddot{q}+C \dot{q}+K q=P
$$

This is the equation of motion, where

$$
\begin{aligned}
& M=\int_{0}^{a} \int_{0}^{b} w^{2}{ }_{m n}(x, y) \rho h d x d y \\
& C=\int_{0}^{a} \int_{0}^{b} w_{m n}^{2}(x, y) c_{b} d x d y \\
& K=\int_{0}^{a} \int_{0}^{b}\left[D_{x}\left(\frac{\partial^{2} w_{m n}}{\partial x^{2}}\right)^{2}+\left(D_{x} v_{y x}+D_{y} v_{x y}\right) \frac{\partial^{2} w_{m n}}{\partial x^{2}} \frac{\partial^{2} w_{m n}}{\partial y^{2}}+D_{y}\left(\frac{\partial^{2} w_{m n}}{\partial y^{2}}\right)^{2}+4 D_{x y}\left(\frac{\partial^{2} w_{m n}}{\partial x \partial y}\right)^{2}\right] d x d y \\
& P=\int_{0}^{a} \int_{0}^{b} \sum_{l=1}^{N_{p}} P_{l}(t) \delta\left(x-\hat{x}_{l}(t)\right) \delta\left(y-\hat{y}_{l}(t)\right) w_{m n}(x, y) d x d y
\end{aligned}
$$

This equation of motion is then solved for the generalized coordinate $q_{m n}$ by using a fourth order Runge-Kutta method. 
Normal modes of the plate $w_{m n}(x, y)=Y_{m n}(y) \sin \left(\frac{m \pi x}{a}\right)$ required in the Equation (5.5) are obtained from the Eigen-value analysis of the orthotropic plate.

The governing differential equation of an orthotropic plate for free vibrations can be obtained from Equation (5.6). Since there is no moving load acting on the plate, there is no external work done and for the simplicity of the problem it has been considered that there is no work done due to damping in the plate. So we just have two terms left in the Equation (5.6), which are the strain energy and the kinetic energy.

Which gives us the differential equation as

$$
D_{x} \frac{\partial^{4} w}{\partial x^{4}}+\left(D_{x} v_{y x}+D_{y} v_{x y}\right) \frac{\partial^{4} w}{\partial x^{2} \partial y^{2}}+D_{y} \frac{\partial^{4} w}{\partial y^{4}}+4 D_{x y} \frac{\partial^{4} w}{\partial x^{2} \partial y^{2}}+\rho h \frac{\partial^{2} w}{\partial t^{2}}=0
$$

By using the Betti's law of reciprocity, as per the design manual for orthotropic steel plate deck bridges [13], we get

$$
\frac{D_{x}}{D_{y}}=\frac{v_{x y}}{v_{y x}} \text { we get, } D_{x} v_{y x}=D_{y} v_{x y}
$$

By substituting,

$$
H=D_{x} v_{y x}+2 D_{x y}
$$

Equation (5.14) becomes,

$$
D_{x} \frac{\partial^{4} w}{\partial x^{4}}+2 H \frac{\partial^{4} w}{\partial x^{2} \partial y^{2}}+D_{y} \frac{\partial^{4} w}{\partial y^{4}}+\rho h \frac{\partial^{2} w}{\partial t^{2}}=0
$$

\section{V-b: Eigen-value Analysis of an Orthotropic Plate}

The governing differential equation of an orthotropic plate is

$$
D_{x} \frac{\partial^{4} w(x, y, t)}{\partial x^{4}}+2 H \frac{\partial^{4} w(x, y, t)}{\partial x^{2} \partial y^{2}}+D_{y} \frac{\partial^{4} w(x, y, t)}{\partial y^{4}}+\rho h \frac{\partial^{2} w(x, y, t)}{\partial t^{2}}=0
$$


The displacement function $w(x, y, t)$ can be expressed as the product of two functions, one involving only the space coordinates $x$ and $y$ and the other involving the variable time.

$$
w(x, y, t)=w_{m n}(x, y) * q_{m n}(t)
$$

Where, $q_{m n}(t)$ is assumed to have the solution in terms of $e^{i \omega t}$

By substituting (5.17) into (5.16), we get

$$
D_{x} \frac{\partial^{4} w(x, y)}{\partial x^{4}}+2 H \frac{\partial^{4} w(x, y)}{\partial x^{2} \partial y^{2}}+D_{y} \frac{\partial^{4} w(x, y)}{\partial y^{4}}-\rho h \omega^{2} w(x, y)=0
$$

This is a homogeneous partial differential equation involving the mode shape expression $w(x, y)$, the plate properties, and the circular frequency of oscillations $\omega$.

To make this equation dimensionless, let us introduce the dimensionless space variables $\xi=x / a, \eta=y / b$, where $a$ and $b$ are the plate dimensions and $\phi=b / a$. Equation (5.18) becomes

$$
\phi^{4} D_{x} \frac{\partial^{4} w(\xi, \eta)}{\partial \xi^{4}}+2 \phi^{2} H \frac{\partial^{4} w(\xi, \eta)}{\partial \xi^{2} \partial \eta^{2}}+D_{y} \frac{\partial^{4} w(\xi, \eta)}{\partial \eta^{4}}-\phi^{4} \rho h \omega^{2} a^{4} w(\xi, \eta)=0
$$

i.e.

$$
\frac{\partial^{4} w(\xi, \eta)}{\partial \eta^{4}}+2 \phi^{2} \frac{H}{D_{y}} \frac{\partial^{4} w(\xi, \eta)}{\partial \xi^{2} \partial \eta^{2}}+\phi^{4} \frac{D_{x}}{D_{y}} \frac{\partial^{4} w(\xi, \eta)}{\partial \xi^{4}}-\phi^{4} \frac{\rho h \omega^{2} a^{4}}{D_{y}} w(\xi, \eta)=0
$$

By substituting

$$
\begin{aligned}
& \lambda^{2}=\omega a^{2} \sqrt{\frac{\rho h}{D_{y}}} \quad \text { Which is a plate eigenvalue. } \\
& \frac{\partial^{4} w(\xi, \eta)}{\partial \eta^{4}}+2 \phi^{2} \frac{H}{D_{y}} \frac{\partial^{4} w(\xi, \eta)}{\partial \xi^{2} \partial \eta^{2}}+\phi^{4} \frac{D_{x}}{D_{y}} \frac{\partial^{4} w(\xi, \eta)}{\partial \xi^{4}}-\phi^{4} \lambda^{4} w(\xi, \eta)=0
\end{aligned}
$$


Levy-type solution for the free vibration analysis of rectangular plates:

Let us consider a plate with simple support along edges $\xi=0$, and $\xi=1$

We can express the solution to Equation (5.19) in the form

$$
w(\xi, \eta)=\sum_{m=1}^{k} Y_{m}(\eta) \sin m \pi \xi
$$

By substituting (5.20) into Equation (5.19), we get

$$
\sum_{m=1}^{\infty}\left[\frac{d^{4} Y_{m}(\eta)}{d \eta^{4}}-2 \phi^{2} \frac{H}{D_{y}}(m \pi)^{2} \frac{d^{2} Y_{m}(\eta)}{d \eta^{2}}+\phi^{4} \frac{D_{x}}{D_{y}}(m \pi)^{4} Y_{m}(\eta)-\phi^{4} \lambda^{4} Y_{m}(\eta)\right] \sin m \pi \xi=0
$$

Gives us,

$\frac{d^{4} Y_{m}(\eta)}{d \eta^{4}}-2 \phi^{2} C_{1} \frac{d^{2} Y_{m}(\eta)}{d \eta^{2}}+\phi^{4}\left[C_{2}-\lambda^{4}\right] Y_{m}(\eta)=0$

Where,

$$
C_{1}=\frac{H}{D_{y}}(m \pi)^{2} \text { and } C_{2}=\frac{D_{x}}{D_{y}}(m \pi)^{4}
$$

Equation (5.22) is an ordinary fourth-order homogeneous differential equation with constant coefficients. Assuming the solution of this equation in the form,

$Y_{m}=C e^{\beta \eta}$

$\beta= \pm\left(\phi^{2}\left[C_{1} \pm \sqrt{C_{1}^{2}-C_{2}+\lambda^{4}}\right)^{1 / 2}\right.$

i.e.

$$
\beta= \pm \phi\left(C_{1} \pm \sqrt{C_{1}^{2}-C_{2}+\lambda^{4}}\right)^{1 / 2}
$$


Roots of the solution gives us two different categories depending if $C_{1}^{2}-C_{2}+\lambda^{4}$ is positive or negative.

\section{Category I:}

This category represents the solution when the quantity in the inner square root sign has real roots.

If $C_{2}-C_{1}^{2}<\lambda^{4}$

This category leads us to two other subcategories

\section{Category I(a):}

In this category the quantity in the outer square root sign has real roots.

$$
\text { If } C_{1}>\sqrt{C_{1}^{2}-C_{2}+\lambda^{4}}
$$

The corresponding solution is given by Gorman D. J. [14] as

$$
Y_{m}(\eta)=A_{m} \cosh \beta_{m} \eta+B_{m} \sinh \beta_{m} \eta+C_{m} \sinh \gamma_{m} \eta+D_{m} \cosh \gamma_{m} \eta
$$

Category I(b):

In this category the quantity in the outer square root has imaginary roots.

$$
\text { If } C_{1}<\sqrt{C_{1}^{2}-C_{2}+\lambda^{4}}
$$

The corresponding solution is given by Gorman D. J. [14] as

$$
Y_{m}(\eta)=A_{m} \cosh \beta_{m} \eta+B_{m} \sinh \beta_{m} \eta+C_{m} \sin \gamma_{m} \eta+D_{m} \cos \gamma_{m} \eta
$$

where,

$$
\begin{aligned}
& \beta_{m}=\phi \sqrt{C_{1}+\sqrt{C_{1}^{2}-C_{2}+\lambda^{4}}} \\
& \gamma_{m}=\phi \sqrt{C_{1}-\sqrt{C_{1}^{2}-C_{2}+\lambda^{4}}} \text { or } \gamma_{m}=\phi \sqrt{\sqrt{C_{1}^{2}-C_{2}+\lambda^{4}}-C_{1}}
\end{aligned}
$$


whichever is real

The quantities $A_{m}, B_{m}, C_{m}$ and $D_{m}$ are constants to be determined by means of boundary conditions.

If we locate the $\xi$ axis along the center of the plate, we can divide the possible mode shapes into two types, symmetric or anti-symmetric modes.

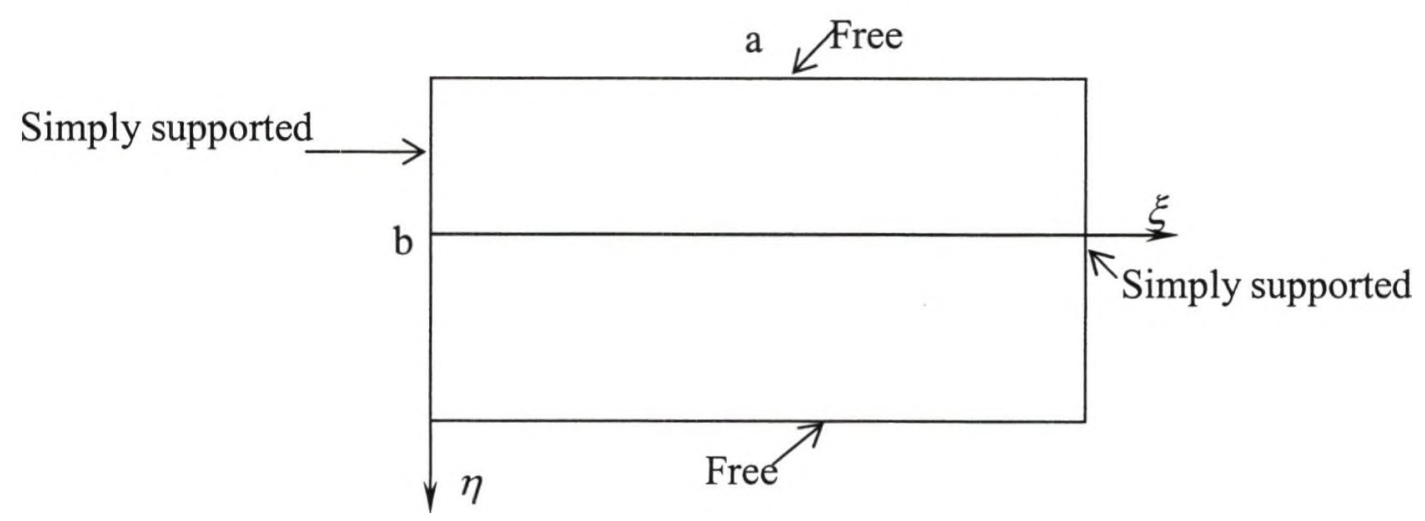

Figure 12: Plate with new axis system.

Symmetric modes:

Restricting to only symmetric modes, we can delete the anti-symmetric terms from Equations (5.24) and (5.25).

$$
\begin{aligned}
& \text { for } C_{1}>\sqrt{C_{1}^{2}-C_{2}+\lambda^{4}} \\
& Y_{m}(\eta)=A_{m} \cosh \beta_{m} \eta+D_{m} \cosh \gamma_{m} \eta \\
& \text { for } C_{1}<\sqrt{C_{1}^{2}-C_{2}+\lambda^{4}} \\
& Y_{m}(\eta)=A_{m} \cosh \beta_{m} \eta+D_{m} \cos \gamma_{m} \eta
\end{aligned}
$$

By using the boundary conditions given by Jayaraman G. et. al. [6] at the free edges at $\eta= \pm \frac{1}{2}$, where bending moment and shear force is zero. 


$$
\begin{aligned}
& \frac{\partial^{2} w(\xi, \eta)}{\partial \eta^{2}}+v_{x y} \phi^{2} \frac{\partial^{2} w(\xi, \eta)}{\partial \xi^{2}}=\left.0\right|_{\eta=1 / 2} \\
& \frac{\partial^{3} w(\xi, \eta)}{\partial \eta^{3}}+v^{*} \phi^{2} \frac{\partial^{3} w(\xi, \eta)}{\partial \eta \partial \xi^{2}}=\left.0\right|_{\eta=1 / 2}
\end{aligned}
$$

Where,

$$
v^{*}=2\left(\frac{H}{D_{y}}\right)-v_{x y}
$$

For $C_{1}>\sqrt{C_{1}^{2}-C_{2}+\lambda^{4}}$

By substituting (5.27) into (5.20), we get the solution as

$$
\begin{aligned}
& w(\xi, \eta)=\left(A_{m} \cosh \beta_{m} \eta+D_{m} \cosh \gamma_{m} \eta\right) \sin m \pi \xi \\
& \frac{\partial w(\xi, \eta)}{\partial \eta}=\left(A_{m} \beta_{m} \sinh \beta_{m} \eta+D_{m} \gamma_{m} \sinh \gamma_{m} \eta\right) \sin m \pi \xi \\
& \frac{\partial^{2} w(\xi, \eta)}{\partial \eta^{2}}=\left(A_{m} \beta_{m}^{2} \cosh \beta_{m} \eta+D_{m} \gamma_{m}^{2} \cosh \gamma_{m} \eta\right) \sin m \pi \xi \\
& \frac{\partial^{3} w(\xi, \eta)}{\partial \eta^{3}}=\left(A_{m} \beta_{m}^{3} \sinh \beta_{m} \eta+D_{m} \gamma_{m}^{3} \sinh \gamma_{m} \eta\right) \sin m \pi \xi \\
& \frac{\partial w(\xi, \eta)}{\partial \xi}=(m \pi)\left(A_{m} \cosh \beta_{m} \eta+D_{m} \cosh \gamma_{m} \eta\right) \cos m \pi \xi \\
& \frac{\partial^{2} w(\xi, \eta)}{\partial \xi^{2}}=(m \pi)^{2}\left(A_{m} \cosh \beta_{m} \eta+D_{m} \cosh \gamma_{m} \eta\right)(-\sin m \pi \xi) \\
& \frac{\partial^{3} w(\xi, \eta)}{\partial \eta \partial \xi^{2}}=(m \pi)^{2}\left(A_{m} \beta_{m} \sinh \beta_{m} \eta+D_{m} \gamma_{m} \sinh \gamma_{m} \eta\right)(-\sin m \pi \xi)
\end{aligned}
$$

Equation (5.29) becomes 


$$
\begin{aligned}
& \left(A_{m} \beta_{m}^{2} \cosh \frac{1}{2} \beta_{m}+D_{m} \gamma_{m}^{2} \cosh \frac{1}{2} \gamma_{m}\right) \sin m \pi \xi \\
& \quad+v_{x y} \phi^{2}(m \pi)^{2}\left(A_{m} \cosh \frac{1}{2} \beta_{m}+D_{m} \cosh \frac{1}{2} \gamma_{m}\right)(-\sin m \pi \xi)=0
\end{aligned}
$$

i.e

$$
A_{m}\left[\beta_{m}^{2}-v_{x y} \phi^{2}(m \pi)^{2}\right] \cosh \frac{1}{2} \beta_{m}+D_{m}\left[\gamma_{m}^{2}-v_{x y} \phi^{2}(m \pi)^{2}\right] \cosh \frac{1}{2} \gamma_{m}=0
$$

and

$$
\begin{aligned}
\left(A_{m} \beta_{m}^{3} \sinh \frac{1}{2} \beta_{m}\right. & \left.+D_{m} \gamma_{m}^{3} \sinh \frac{1}{2} \gamma_{m}\right) \sin m \pi \xi \\
& +v^{*} \phi^{2}(m \pi)^{2}\left(A_{m} \beta_{m} \sinh \frac{1}{2} \beta_{m}+D_{m} \gamma_{m} \sinh \frac{1}{2} \gamma_{m}\right)(-\sin m \pi \xi)=0
\end{aligned}
$$

i.e.

$$
A_{m} \beta_{m}\left[\beta_{m}^{2}-v^{*} \phi^{2}(m \pi)^{2}\right] \sinh \frac{1}{2} \beta_{m}+D_{m} \gamma_{m}\left[\gamma_{m}^{2}-v^{*} \phi^{2}(m \pi)^{2}\right] \sinh \frac{1}{2} \gamma_{m}=0
$$

Equations (5.30) and (5.31) can be written as,

$$
\left[\begin{array}{cc}
{\left[\beta_{m}^{2}-v_{x y} \phi^{2}(m \pi)^{2}\right] \cosh \frac{1}{2} \beta_{m}} & {\left[\gamma_{m}^{2}-v_{x y} \phi^{2}(m \pi)^{2}\right] \cosh \frac{1}{2} \gamma_{m}} \\
\beta_{m}\left[\beta_{m}^{2}-v^{*} \phi^{2}(m \pi)^{2}\right] \sinh \frac{1}{2} \beta_{m} & \gamma_{m}\left[\gamma_{m}^{2}-v^{*} \phi^{2}(m \pi)^{2}\right] \sinh \frac{1}{2} \gamma_{m}
\end{array}\right]\left[\begin{array}{l}
A_{m} \\
D_{m}
\end{array}\right]=\left[\begin{array}{l}
0 \\
0
\end{array}\right]
$$

To satisfy Equation (5.32), determinant of the first term has to be equal to zero.

$$
\left|\begin{array}{cc}
{\left[\beta_{m}^{2}-v_{x y} \phi^{2}(m \pi)^{2}\right] \cosh \frac{1}{2} \beta_{m}} & {\left[\gamma_{m}^{2}-v_{x y} \phi^{2}(m \pi)^{2}\right] \cosh \frac{1}{2} \gamma_{m}} \\
\beta_{m}\left[\beta_{m}^{2}-v^{*} \phi^{2}(m \pi)^{2}\right] \sinh \frac{1}{2} \beta_{m} & \gamma_{m}\left[\gamma_{m}^{2}-v^{*} \phi^{2}(m \pi)^{2}\right] \sinh \frac{1}{2} \gamma_{m}
\end{array}\right|=0
$$

Which gives us the Eigen-value equation as 


$$
\begin{aligned}
\gamma_{m}\left[\gamma_{m}^{2}-v^{*} \phi^{2}(m \pi)^{2}\right] \cdot\left[\beta_{m}^{2}-v_{x y} \phi^{2}(m \pi)^{2}\right] \sinh \frac{1}{2} \gamma_{m} \cosh \frac{1}{2} \beta_{m} \\
-\beta_{m}\left[\beta_{m}^{2}-v^{*} \phi^{2}(m \pi)^{2}\right] \cdot\left[\gamma_{m}^{2}-v_{x y} \phi^{2}(m \pi)^{2}\right] \sinh \frac{1}{2} \beta_{m} \cosh \frac{1}{2} \gamma_{m}=0
\end{aligned}
$$

By substituting $\beta_{m}$ and $\gamma_{m}$ in terms of $\lambda$ in Equation (5.33) and solving for different values of $\mathrm{m}$, gives us different eigenvalues $\lambda$.

By setting $D_{m}=1$ in Equation (5.30), we get $A_{m}$ as

$$
A_{m}=-\frac{\left[\gamma_{m}^{2}-v_{x y} \phi^{2}(m \pi)^{2}\right] \cosh \frac{1}{2} \gamma_{m}}{\left[\beta_{m}^{2}-v_{x y} \phi^{2}(m \pi)^{2}\right] \cosh \frac{1}{2} \beta_{m}}
$$

By substituting $D_{m}$ and $A_{m}$ in Equation (5.27) and then into Equation (5.20), we get mode shape of the plate as

$$
w(\xi, \eta)=\left[\cosh \gamma_{m} \eta-\frac{\left[\gamma_{m}^{2}-v_{x y} \phi^{2}(m \pi)^{2}\right] \cosh \frac{1}{2} \gamma_{m}}{\left[\beta_{m}^{2}-v_{x y} \phi^{2}(m \pi)^{2}\right] \cosh \frac{1}{2} \beta_{m}} \cosh \beta_{m} \eta\right] \sin m \pi \xi
$$

For $C_{1}<\sqrt{C_{1}^{2}-C_{2}+\lambda^{4}}$

By substituting (5.28) into (5.20), we get the solution as

$$
\begin{aligned}
& w(\xi, \eta)=\left(A_{m} \cosh \beta_{m} \eta+D_{m} \cos \gamma_{m} \eta\right) \sin m \pi \xi \\
& \frac{\partial w(\xi, \eta)}{\partial \eta}=\left(A_{m} \beta_{m} \sinh \beta_{m} \eta-D_{m} \gamma_{m} \sin \gamma_{m} \eta\right) \sin m \pi \xi \\
& \frac{\partial^{2} w(\xi, \eta)}{\partial \eta^{2}}=\left(A_{m} \beta_{m}^{2} \cosh \beta_{m} \eta-D_{m} \gamma_{m}^{2} \cos \gamma_{m} \eta\right) \sin m \pi \xi \\
& \frac{\partial^{3} w(\xi, \eta)}{\partial \eta^{3}}=\left(A_{m} \beta_{m}^{3} \sinh \beta_{m} \eta+D_{m} \gamma_{m}^{3} \sin \gamma_{m} \eta\right) \sin m \pi \xi
\end{aligned}
$$




$$
\begin{aligned}
& \frac{\partial w(\xi, \eta)}{\partial \xi}=(m \pi)\left(A_{m} \cosh \beta_{m} \eta+D_{m} \cos \gamma_{m} \eta\right) \cos m \pi \xi \\
& \frac{\partial^{2} w(\xi, \eta)}{\partial \xi^{2}}=(m \pi)^{2}\left(A_{m} \cosh \beta_{m} \eta+D_{m} \cos \gamma_{m} \eta\right)(-\sin m \pi \xi) \\
& \frac{\partial^{3} w(\xi, \eta)}{\partial \eta \partial \xi^{2}}=(m \pi)^{2}\left(A_{m} \beta_{m} \sinh \beta_{m} \eta-D_{m} \gamma_{m} \sin \gamma_{m} \eta\right)(-\sin m \pi \xi)
\end{aligned}
$$

Equation (5.29) becomes

$$
\begin{aligned}
& \left(A_{m} \beta_{m}^{2} \cosh \frac{1}{2} \beta_{m}-D_{m} \gamma_{m}^{2} \cos \frac{1}{2} \gamma_{m}\right) \sin m \pi \xi \\
& \quad+v_{x y} \phi^{2}(m \pi)^{2}\left(A_{m} \cosh \frac{1}{2} \beta_{m}+D_{m} \cos \frac{1}{2} \gamma_{m}\right)(-\sin m \pi \xi)=0
\end{aligned}
$$

i.e

$$
A_{m}\left[\beta_{m}^{2}-v_{x y} \phi^{2}(m \pi)^{2}\right] \cosh \frac{1}{2} \beta_{m}-D_{m}\left[\gamma_{m}^{2}+v_{x y} \phi^{2}(m \pi)^{2}\right] \cos \frac{1}{2} \gamma_{m}=0
$$

and

$$
\begin{aligned}
\left(A_{m} \beta_{m}^{3} \sinh \frac{1}{2} \beta_{m}\right. & \left.+D_{m} \gamma_{m}^{3} \sin \frac{1}{2} \gamma_{m}\right) \sin m \pi \xi \\
& +v^{*} \phi^{2}(m \pi)^{2}\left(A_{m} \beta_{m} \sinh \frac{1}{2} \beta_{m}-D_{m} \gamma_{m} \sin \frac{1}{2} \gamma_{m}\right)(-\sin m \pi \xi)=0
\end{aligned}
$$

i.e.

$$
A_{m} \beta_{m}\left[\beta_{m}^{2}-v^{*} \phi^{2}(m \pi)^{2}\right] \sinh \frac{1}{2} \beta_{m}+D_{m} \gamma_{m}\left[\gamma_{m}^{2}+v^{*} \phi^{2}(m \pi)^{2}\right] \sin \frac{1}{2} \gamma_{m}=0
$$

Equations (5.35) and (5.36) can be written as

$$
\left[\begin{array}{cc}
{\left[\beta_{m}^{2}-v_{x y} \phi^{2}(m \pi)^{2}\right] \cosh \frac{1}{2} \beta_{m}} & -\left[\gamma_{m}^{2}+v_{x y} \phi^{2}(m \pi)^{2}\right] \cos \frac{1}{2} \gamma_{m} \\
\beta_{m}\left[\beta_{m}^{2}-v^{*} \phi^{2}(m \pi)^{2}\right] \sinh \frac{1}{2} \beta_{m} & \gamma_{m}\left[\gamma_{m}^{2}+v^{*} \phi^{2}(m \pi)^{2}\right] \sin \frac{1}{2} \gamma_{m}
\end{array}\right]\left[\begin{array}{l}
A_{m} \\
D_{m}
\end{array}\right]=\left[\begin{array}{l}
0 \\
0
\end{array}\right]
$$

48 
To satisfy Equation (5.37), determinant of the first term has to be equal to zero.

$$
\left|\begin{array}{ll}
{\left[\beta_{m}^{2}-v_{x y} \phi^{2}(m \pi)^{2}\right] \cosh \frac{1}{2} \beta_{m}} & -\left[\gamma_{m}^{2}+v_{x y} \phi^{2}(m \pi)^{2}\right] \cos \frac{1}{2} \gamma_{m} \\
\beta_{m}\left[\beta_{m}^{2}-v^{*} \phi^{2}(m \pi)^{2}\right] \sinh \frac{1}{2} \beta_{m} & \gamma_{m}\left[\gamma_{m}^{2}+v^{*} \phi^{2}(m \pi)^{2}\right] \sin \frac{1}{2} \gamma_{m}
\end{array}\right|=0
$$

Which gives us the Eigen-value equation as

$$
\begin{aligned}
\gamma_{m}\left[\gamma_{m}^{2}+v^{*} \phi^{2}(m \pi)^{2}\right] \cdot\left[\beta_{m}^{2}-v_{x y} \phi^{2}(m \pi)^{2}\right] \sin \frac{1}{2} \gamma_{m} \cosh \frac{1}{2} \beta_{m} \\
\quad+\beta_{m}\left[\beta_{m}^{2}-v^{*} \phi^{2}(m \pi)^{2}\right] \cdot\left[\gamma_{m}^{2}+v_{x y} \phi^{2}(m \pi)^{2}\right] \sinh \frac{1}{2} \beta_{m} \cos \frac{1}{2} \gamma_{m}=0
\end{aligned}
$$

By substituting $\beta_{m}$ and $\gamma_{m}$ in terms of $\lambda$ in Equation (5.38) and solving for different values of $\mathrm{m}$, gives us different eigenvalues $\lambda$.

By setting $D_{m}=1$ in Equation (5.35), we get $A_{m}$ as

$$
A_{m}=\frac{\left[\gamma_{m}^{2}+v_{x y} \phi^{2}(m \pi)^{2}\right] \cos \frac{1}{2} \gamma_{m}}{\left[\beta_{m}^{2}-v_{x y} \phi^{2}(m \pi)^{2}\right] \cosh \frac{1}{2} \beta_{m}}
$$

By substituting $D_{m}$ and $A_{m}$ in Equation (5.28) and then into Equation (5.20), we get mode shape of the plate as

$$
w(\xi, \eta)=\left[\cos \gamma_{m} \eta+\frac{\left[\gamma_{m}^{2}+v_{x y} \phi^{2}(m \pi)^{2}\right] \cos \frac{1}{2} \gamma_{m}}{\left[\beta_{m}^{2}-v_{x y} \phi^{2}(m \pi)^{2}\right] \cosh \frac{1}{2} \beta_{m}} \cosh \beta_{m} \eta\right] \sin m \pi \xi
$$

Anti-symmetric modes:

Restricting to only anti-symmetric modes, we can delete the symmetric terms from Equations (5.24) and (5.25).

$$
\text { For } C_{1}>\sqrt{C_{1}^{2}-C_{2}+\lambda^{4}}
$$




$$
Y_{m}(\eta)=B_{m} \sinh \beta_{m} \eta+C_{m} \sinh \gamma_{m} \eta
$$

For $C_{1}<\sqrt{C_{1}^{2}-C_{2}+\lambda^{4}}$

$$
Y_{m}(\eta)=B_{m} \sinh \beta_{m} \eta+C_{m} \sin \gamma_{m} \eta
$$

For $C_{1}>\sqrt{C_{1}^{2}-C_{2}+\lambda^{4}}$

By substituting (5.40) into (5.20), we get the solution as

$$
\begin{aligned}
& w(\xi, \eta)=\left(B_{m} \sinh \beta_{m} \eta+C_{m} \sinh \gamma_{m} \eta\right) \sin m \pi \xi \\
& \frac{\partial w(\xi, \eta)}{\partial \eta}=\left(B_{m} \beta_{m} \cosh \beta_{m} \eta+C_{m} \gamma_{m} \cosh \gamma_{m} \eta\right) \sin m \pi \xi \\
& \frac{\partial^{2} w(\xi, \eta)}{\partial \eta^{2}}=\left(B_{m} \beta_{m}^{2} \sinh \beta_{m} \eta+C_{m} \gamma_{m}^{2} \sinh \gamma_{m} \eta\right) \sin m \pi \xi \\
& \frac{\partial^{3} w(\xi, \eta)}{\partial \eta^{3}}=\left(B_{m} \beta_{m}^{3} \cosh \beta_{m} \eta+C_{m} \gamma_{m}^{3} \cosh \gamma_{m} \eta\right) \sin m \pi \xi \\
& \frac{\partial w(\xi, \eta)}{\partial \xi}=(m \pi)\left(B_{m} \sinh \beta_{m} \eta+C_{m} \sinh \gamma_{m} \eta\right) \cos m \pi \xi \\
& \frac{\partial^{2} w(\xi, \eta)}{\partial \xi^{2}}=(m \pi)^{2}\left(B_{m} \sinh \beta_{m} \eta+C_{m} \sinh \gamma_{m} \eta\right)(-\sin m \pi \xi) \\
& \frac{\partial^{3} w(\xi, \eta)}{\partial \eta \partial \xi^{2}}=(m \pi)^{2}\left(B_{m} \beta_{m} \cosh \beta_{m} \eta+C_{m} \gamma_{m} \cosh \gamma_{m} \eta\right)(-\sin m \pi \xi)
\end{aligned}
$$

Equation (5.29) becomes

$$
\begin{aligned}
\left(B_{m} \beta_{m}^{2} \sinh \frac{1}{2} \beta_{m}+C_{m} \gamma_{m}^{2} \sinh \frac{1}{2} \gamma_{m}\right) \sin m \pi \xi \\
\quad+v_{x y} \phi^{2}(m \pi)^{2}\left(B_{m} \sinh \frac{1}{2} \beta_{m}+C_{m} \sinh \frac{1}{2} \gamma_{m}\right)(-\sin m \pi \xi)=0
\end{aligned}
$$


$B_{m}\left[\beta_{m}^{2}-v_{x y} \phi^{2}(m \pi)^{2}\right] \sinh \frac{1}{2} \beta_{m}+C_{m}\left[\gamma_{m}^{2}-v_{x y} \phi^{2}(m \pi)^{2}\right] \sinh \frac{1}{2} \gamma_{m}=0$

and

$$
\begin{aligned}
\left(B_{m} \beta_{m}^{3} \cosh \frac{1}{2} \beta_{m}\right. & \left.+C_{m} \gamma_{m}^{3} \cosh \frac{1}{2} \gamma_{m}\right) \sin m \pi \xi \\
& +v^{*} \phi^{2}(m \pi)^{2}\left(B_{m} \beta_{m} \cosh \frac{1}{2} \beta_{m}+C_{m} \gamma_{m} \cosh \frac{1}{2} \gamma_{m}\right)(-\sin m \pi \xi)=0
\end{aligned}
$$

i.e.

$$
B_{m} \beta_{m}\left[\beta_{m}^{2}-v^{*} \phi^{2}(m \pi)^{2}\right] \cosh \frac{1}{2} \beta_{m}+C_{m} \gamma_{m}\left[\gamma_{m}^{2}-v^{*} \phi^{2}(m \pi)^{2}\right] \cosh \frac{1}{2} \gamma_{m}=0
$$

Equations (5.42) and (5.43) can be written as

$$
\left[\begin{array}{cc}
{\left[\beta_{m}^{2}-v_{x y} \phi^{2}(m \pi)^{2}\right] \sinh \frac{1}{2} \beta_{m}} & {\left[\gamma_{m}^{2}-v_{x y} \phi^{2}(m \pi)^{2}\right] \sinh \frac{1}{2} \gamma_{m}} \\
\beta_{m}\left[\beta_{m}^{2}-v^{*} \phi^{2}(m \pi)^{2}\right] \cosh \frac{1}{2} \beta_{m} & \gamma_{m}\left[\gamma_{m}^{2}-v^{*} \phi^{2}(m \pi)^{2}\right] \cosh \frac{1}{2} \gamma_{m}
\end{array}\right]\left[\begin{array}{l}
B_{m} \\
C_{m}
\end{array}\right]=\left[\begin{array}{l}
0 \\
0
\end{array}\right]
$$

To satisfy Equation (5.44), determinant of the first term has to be equal to zero.

$$
\left|\begin{array}{cc}
{\left[\beta_{m}^{2}-v_{x y} \phi^{2}(m \pi)^{2}\right] \sinh \frac{1}{2} \beta_{m}} & {\left[\gamma_{m}^{2}-v_{x y} \phi^{2}(m \pi)^{2}\right] \sinh \frac{1}{2} \gamma_{m}} \\
\beta_{m}\left[\beta_{m}^{2}-v^{*} \phi^{2}(m \pi)^{2}\right] \cosh \frac{1}{2} \beta_{m} & \gamma_{m}\left[\gamma_{m}^{2}-v^{*} \phi^{2}(m \pi)^{2}\right] \cosh \frac{1}{2} \gamma_{m}
\end{array}\right|=0
$$

Which gives us the Eigen-value equation as

$$
\begin{aligned}
\gamma_{m}\left[\gamma_{m}^{2}-v^{*} \phi^{2}(m \pi)^{2}\right] \cdot\left[\beta_{m}^{2}-v_{x y} \phi^{2}(m \pi)^{2}\right] \cosh \frac{1}{2} \gamma_{m} \sinh \frac{1}{2} \beta_{m} \\
-\beta_{m}\left[\beta_{m}^{2}-v^{*} \phi^{2}(m \pi)^{2}\right] \cdot\left[\gamma_{m}^{2}-v_{x y} \phi^{2}(m \pi)^{2}\right] \cosh \frac{1}{2} \beta_{m} \sinh \frac{1}{2} \gamma_{m}=0
\end{aligned}
$$

By substituting $\beta_{m}$ and $\gamma_{m}$ in terms of $\lambda$ in Equation (5.45) and solving for different values of $\mathrm{m}$, gives us different eigenvalues $\lambda$. 
By setting $C_{m}=1$ in Equation (5.42), we get $B_{m}$ as

$$
B_{m}=-\frac{\left[\gamma_{m}^{2}-v_{x y} \phi^{2}(m \pi)^{2}\right] \sinh \frac{1}{2} \gamma_{m}}{\left[\beta_{m}^{2}-v_{x y} \phi^{2}(m \pi)^{2}\right] \sinh \frac{1}{2} \beta_{m}}
$$

By substituting $C_{m}$ and $B_{m}$ in Equation (5.40) and then into Equation (5.20), we get mode shape of the plate as

$w(\xi, \eta)=\left[\sinh \gamma_{m} \eta-\frac{\left[\gamma_{m}^{2}-v_{x y} \phi^{2}(m \pi)^{2}\right] \sinh \frac{1}{2} \gamma_{m}}{\left[\beta_{m}^{2}-v_{x y} \phi^{2}(m \pi)^{2}\right] \sinh \frac{1}{2} \beta_{m}} \sinh \beta_{m} \eta\right] \sin m \pi \xi$

For $C_{1}<\sqrt{C_{1}^{2}-C_{2}+\lambda^{4}}$

By substituting (5.41) into (5.20), we get the solution as

$$
\begin{aligned}
& w(\xi, \eta)=\left(B_{m} \sinh \beta_{m} \eta+C_{m} \sin \gamma_{m} \eta\right) \sin m \pi \xi \\
& \frac{\partial w(\xi, \eta)}{\partial \eta}=\left(B_{m} \beta_{m} \cosh \beta_{m} \eta+C_{m} \gamma_{m} \cos \gamma_{m} \eta\right) \sin m \pi \xi \\
& \frac{\partial^{2} w(\xi, \eta)}{\partial \eta^{2}}=\left(B_{m} \beta_{m}^{2} \sinh \beta_{m} \eta-C_{m} \gamma_{m}^{2} \sin \gamma_{m} \eta\right) \sin m \pi \xi \\
& \frac{\partial^{3} w(\xi, \eta)}{\partial \eta^{3}}=\left(B_{m} \beta_{m}^{3} \cosh \beta_{m} \eta-C_{m} \gamma_{m}^{3} \cos \gamma_{m} \eta\right) \sin m \pi \xi \\
& \frac{\partial w(\xi, \eta)}{\partial \xi}=(m \pi)\left(B_{m} \sinh \beta_{m} \eta+C_{m} \sin \gamma_{m} \eta\right) \cos m \pi \xi \\
& \frac{\partial^{2} w(\xi, \eta)}{\partial \xi^{2}}=(m \pi)^{2}\left(B_{m} \sinh \beta_{m} \eta+C_{m} \sin \gamma_{m} \eta\right)(-\sin m \pi \xi) \\
& \frac{\partial^{3} w(\xi, \eta)}{\partial \eta \partial \xi^{2}}=(m \pi)^{2}\left(B_{m} \beta_{m} \cosh \beta_{m} \eta+C_{m} \gamma_{m} \cos \gamma_{m} \eta\right)(-\sin m \pi \xi)
\end{aligned}
$$

Equation (5.29) becomes 


$$
\begin{aligned}
\left(B_{m} \beta_{m}^{2} \sinh \frac{1}{2} \beta_{m}-C_{m} \gamma_{m}^{2} \sin \frac{1}{2} \gamma_{m}\right) \sin m \pi \xi \\
\quad+v_{x y} \phi^{2}(m \pi)^{2}\left(B_{m} \sinh \frac{1}{2} \beta_{m}+C_{m} \sin \frac{1}{2} \gamma_{m}\right)(-\sin m \pi \xi)=0
\end{aligned}
$$

i.e

$B_{m}\left[\beta_{m}^{2}-v_{x y} \phi^{2}(m \pi)^{2}\right] \sinh \frac{1}{2} \beta_{m}-C_{m}\left[\gamma_{m}^{2}+v_{x y} \phi^{2}(m \pi)^{2}\right] \sin \frac{1}{2} \gamma_{m}=0$

and

$$
\begin{aligned}
\left(B_{m} \beta_{m}^{3} \cosh \frac{1}{2} \beta_{m}-C_{m} \gamma_{m}^{3} \cos \frac{1}{2} \gamma_{m}\right) \sin m \pi \xi \\
\quad+v^{*} \phi^{2}(m \pi)^{2}\left(B_{m} \beta_{m} \cosh \frac{1}{2} \beta_{m}+C_{m} \gamma_{m} \cos \frac{1}{2} \gamma_{m}\right)(-\sin m \pi \xi)=0
\end{aligned}
$$

i.e.

$$
B_{m} \beta_{m}\left[\beta_{m}^{2}-v^{*} \phi^{2}(m \pi)^{2}\right] \cosh \frac{1}{2} \beta_{m}-C_{m} \gamma_{m}\left[\gamma_{m}^{2}+v^{*} \phi^{2}(m \pi)^{2}\right] \cos \frac{1}{2} \gamma_{m}=0
$$

Equations (5.47) and (5.48) can be written as

$$
\left[\begin{array}{cc}
{\left[\beta_{m}^{2}-v_{x y} \phi^{2}(m \pi)^{2}\right] \sinh \frac{1}{2} \beta_{m}} & -\left[\gamma_{m}^{2}+v_{x y} \phi^{2}(m \pi)^{2}\right] \sin \frac{1}{2} \gamma_{m} \\
\beta_{m}\left[\beta_{m}^{2}-v^{*} \phi^{2}(m \pi)^{2}\right] \cosh \frac{1}{2} \beta_{m} & -\gamma_{m}\left[\gamma_{m}^{2}+v^{*} \phi^{2}(m \pi)^{2}\right] \cos \frac{1}{2} \gamma_{m}
\end{array}\right]\left[\begin{array}{l}
B_{m} \\
C_{m}
\end{array}\right]=\left[\begin{array}{l}
0 \\
0
\end{array}\right]
$$

To satisfy Equation (5.49), determinant of the first term has to be equal to zero.

$$
\left|\begin{array}{cc}
{\left[\beta_{m}^{2}-v_{x y} \phi^{2}(m \pi)^{2}\right] \sinh \frac{1}{2} \beta_{m}} & -\left[\gamma_{m}^{2}+v_{x y} \phi^{2}(m \pi)^{2}\right] \sin \frac{1}{2} \gamma_{m} \\
\beta_{m}\left[\beta_{m}^{2}-v^{*} \phi^{2}(m \pi)^{2}\right] \cosh \frac{1}{2} \beta_{m} & -\gamma_{m}\left[\gamma_{m}^{2}+v^{*} \phi^{2}(m \pi)^{2}\right] \cos \frac{1}{2} \gamma_{m}
\end{array}\right|=0
$$

Which gives us the Eigen-value equation as 


$$
\begin{aligned}
\gamma_{m}\left[\gamma_{m}^{2}+v^{*} \phi^{2}(m \pi)^{2}\right] \cdot\left[\beta_{m}^{2}-v_{x y} \phi^{2}(m \pi)^{2}\right] \cos \frac{1}{2} \gamma_{m} \sinh \frac{1}{2} \beta_{m} \\
-\beta_{m}\left[\beta_{m}^{2}-v^{*} \phi^{2}(m \pi)^{2}\right] \cdot\left[\gamma_{m}^{2}+v_{x y} \phi^{2}(m \pi)^{2}\right] \cosh \frac{1}{2} \beta_{m} \sin \frac{1}{2} \gamma_{m}=0
\end{aligned}
$$

By substituting $\beta_{m}$ and $\gamma_{m}$ in terms of $\lambda$ in Equation (5.50) and solving for different values of $\mathrm{m}$, gives us different eigenvalues $\lambda$.

By setting $C_{m}=1$ in Equation (5.47), we get $B_{m}$ as

$$
B_{m}=+\frac{\left[\gamma_{m}^{2}+v_{x y} \phi^{2}(m \pi)^{2}\right] \sin \frac{1}{2} \gamma_{m}}{\left[\beta_{m}^{2}-v_{x y} \phi^{2}(m \pi)^{2}\right] \sinh \frac{1}{2} \beta_{m}}
$$

By substituting $C_{m}$ and $B_{m}$ in Equation (5.41) and then into Equation (5.20), we get mode shape of the plate as

$$
w(\xi, \eta)=\left[\sin \gamma_{m} \eta+\frac{\left[\gamma_{m}^{2}+v_{x y} \phi^{2}(m \pi)^{2}\right] \sin \frac{1}{2} \gamma_{m}}{\left[\beta_{m}^{2}-v_{x y} \phi^{2}(m \pi)^{2}\right] \sinh \frac{1}{2} \beta_{m}} \sinh \beta_{m} \eta\right] \sin m \pi \xi
$$

Category II:

This category represents the set of solutions when the quantity in the inner square root sign is imaginary.

$$
\text { If } C_{2}-C_{1}^{2}>\lambda^{4}
$$

The corresponding solution for Equation (5.22) is given by Jayaraman G. et. al. [6] as $Y_{m}(\eta)=A_{m} \cosh \beta_{m} \eta \cdot \cos \gamma_{m} \eta+B_{m} \cosh \beta_{m} \eta \cdot \sin \gamma_{m} \eta+C_{m} \sinh \beta_{m} \eta \cdot \cos \gamma_{m} \eta+D_{m} \sinh \beta_{m} \eta \cdot \sin \gamma_{m} \eta$

We can separate the symmetrical modes and anti-symmetrical modes from the above equation. 
For the symmetrical modes, the solution can be written as

$Y_{m}(\eta)=A_{m} \cosh \beta_{m} \eta \cdot \cos \gamma_{m} \eta+D_{m} \sinh \beta_{m} \eta \cdot \sin \gamma_{m} \eta$

And for Anti-symmetrical modes, the solution can be written as,

$Y_{m}(\eta)=B_{m} \cosh \beta_{m} \eta \cdot \sin \gamma_{m} \eta+C_{m} \sinh \beta_{m} \eta \cdot \cos \gamma_{m} \eta$

Symmetrical modes:

The solution (as given in Equation (5.20)) to the differential Equation (5.19) is in the form of $w(\xi, \eta)=\sum_{m=1}^{k} Y_{m}(\eta) \sin m \pi \xi$

For the symmetrical modes, this equation becomes

$w(\xi, \eta)=\left(A_{m} \cosh \beta_{m} \eta \cos \gamma_{m} \eta+D_{m} \sinh \beta_{m} \eta \sin \gamma_{m} \eta\right) \sin m \pi \xi$

$\frac{\partial w(\xi, \eta)}{\partial \eta}=\left(A_{m} \beta_{m} \sinh \beta_{m} \eta \cos \gamma_{m} \eta-A_{m} \gamma_{m} \cosh \beta_{m} \eta \sin \gamma_{m} \eta\right.$

$\left.+D_{m} \beta_{m} \cosh \beta_{m} \eta \sin \gamma_{m} \eta+D_{m} \gamma_{m} \sinh \beta_{m} \eta \cos \gamma_{m} \eta\right) \sin m \pi \xi$

$\frac{\partial^{2} w(\xi, \eta)}{\partial \eta^{2}}=\left(A_{m} \beta_{m}^{2} \cosh \beta_{m} \eta \cos \gamma_{m} \eta-A_{m} \beta_{m} \gamma_{m} \sinh \beta_{m} \eta \sin \gamma_{m} \eta\right.$

$-A_{m} \gamma_{m} \beta_{m} \sinh \beta_{m} \eta \sin \gamma_{m} \eta-A_{m} \gamma_{m}^{2} \cosh \beta_{m} \eta \cos \gamma_{m} \eta$

$+D_{m} \beta_{m}^{2} \sinh \beta_{m} \eta \sin \gamma_{m} \eta+D_{m} \gamma_{m} \beta_{m} \cosh \beta_{m} \eta \cos \gamma_{m} \eta$

$\left.+D_{m} \gamma_{m} \beta_{m} \cosh \beta_{m} \eta \cos \gamma_{m} \eta-D_{m} \gamma_{m}^{2} \sinh \beta_{m} \eta \sin \gamma_{m} \eta\right) \sin m \pi \xi$

$\frac{\partial^{3} w(\xi, \eta)}{\partial \eta^{3}}=\left(A_{m} \beta_{m}^{3} \sinh \beta_{m} \eta \cos \gamma_{m} \eta-A_{m} \beta_{m}^{2} \gamma_{m} \cosh \beta_{m} \eta \sin \gamma_{m} \eta\right.$

$-A_{m} \beta_{m}^{2} \gamma_{m} \cosh \beta_{m} \eta \sin \gamma_{m} \eta-A_{m} \beta_{m} \gamma_{m}^{2} \sinh \beta_{m} \eta \cos \gamma_{m} \eta$

$-A_{m} \gamma_{m} \beta_{m}^{2} \cosh \beta_{m} \eta \sin \gamma_{m} \eta-A_{m} \gamma_{m}^{2} \beta_{m} \sinh \beta_{m} \eta \cos \gamma_{m} \eta$ 
$-A_{m} \gamma_{m}^{2} \beta_{m} \sinh \beta_{m} \eta \cos \gamma_{m} \eta+A_{m} \gamma_{m}^{3} \cosh \beta_{m} \eta \sin \gamma_{m} \eta$

$+D_{m} \beta_{m}^{3} \cosh \beta_{m} \eta \sin \gamma_{m} \eta+D_{m} \gamma_{m} \beta_{m}^{2} \sinh \beta_{m} \eta \cos \gamma_{m} \eta$

$+D_{m} \beta_{m}^{2} \gamma_{m} \sinh \beta_{m} \eta \cos \gamma_{m} \eta-D_{m} \gamma_{m}^{2} \beta_{m} \cosh \beta_{m} \eta \sin \gamma_{m} \eta$

$+D_{m} \gamma_{m} \beta_{m}^{2} \sinh \beta_{m} \eta \cos \gamma_{m} \eta-D_{m} \gamma_{m}^{2} \beta_{m} \cosh \beta_{m} \eta \sin \gamma_{m} \eta$

$\left.-D_{m} \gamma_{m}^{2} \beta_{m} \cosh \beta_{m} \eta \sin \gamma_{m} \eta-D_{m} \gamma_{m}^{3} \sinh \beta_{m} \eta \cos \gamma_{m} \eta\right) \sin m \pi \xi$

$\frac{\partial w(\xi, \eta)}{\partial \xi}=m \pi\left(A_{m} \cosh \beta_{m} \eta \cos \gamma_{m} \eta+D_{m} \sinh \beta_{m} \eta \sin \gamma_{m} \eta\right) \cos m \pi \xi$

$\frac{\partial^{2} w(\xi, \eta)}{\partial \xi^{2}}=-(m \pi)^{2}\left(A_{m} \cosh \beta_{m} \eta \cos \gamma_{m} \eta+D_{m} \sinh \beta_{m} \eta \sin \gamma_{m} \eta\right) \sin m \pi \xi$

$\frac{\partial^{3} w(\xi, \eta)}{\partial \eta \partial \xi^{2}}=-(m \pi)^{2}\left(A_{m} \beta_{m} \sinh \beta_{m} \eta \cos \gamma_{m} \eta-A_{m} \gamma_{m} \cosh \beta_{m} \eta \sin \gamma_{m} \eta\right.$

$\left.+D_{m} \beta_{m} \cosh \beta_{m} \eta \sin \gamma_{m} \eta+D_{m} \gamma_{m} \sinh \beta_{m} \eta \cos \gamma_{m} \eta\right) \sin m \pi \xi$

The boundary conditions of Equation (5.29) become

$$
\begin{aligned}
& {\left[A_{m} \beta_{m}^{2} \cosh \frac{1}{2} \beta_{m} \cos \frac{1}{2} \gamma_{m}-A_{m} \beta_{m} \gamma_{m} \sinh \frac{1}{2} \beta_{m} \sin \frac{1}{2} \gamma_{m}\right.} \\
& -A_{m} \gamma_{m} \beta_{m} \sinh \frac{1}{2} \beta_{m} \sin \frac{1}{2} \gamma_{m}-A_{m} \gamma_{m}^{2} \cosh \frac{1}{2} \beta_{m} \cos \frac{1}{2} \gamma_{m} \\
& +D_{m} \beta_{m}^{2} \sinh \frac{1}{2} \beta_{m} \sin \frac{1}{2} \gamma_{m}+D_{m} \gamma_{m} \beta_{m} \cosh \frac{1}{2} \beta_{m} \cos \frac{1}{2} \gamma_{m} \\
& \left.+D_{m} \gamma_{m} \beta_{m} \cosh \frac{1}{2} \beta_{m} \cos \frac{1}{2} \gamma_{m}-D_{m} \gamma_{m}^{2} \sinh \frac{1}{2} \beta_{m} \sin \frac{1}{2} \gamma_{m}\right] \sin m \pi \xi \\
& -v_{x y} \phi^{2}(m \pi)^{2}\left(A_{m} \cosh \frac{1}{2} \beta_{m} \cos \frac{1}{2} \gamma_{m}+D_{m} \sinh \frac{1}{2} \beta_{m} \sin \frac{1}{2} \gamma_{m}\right) \sin m \pi \xi=0
\end{aligned}
$$

and, 


$$
\begin{aligned}
{\left[A_{m} \beta_{m}^{3} \sinh \frac{1}{2} \beta_{m} \cos \frac{1}{2} \gamma_{m}-A_{m} \beta_{m}^{2} \gamma_{m} \cosh \frac{1}{2} \beta_{m} \sin \frac{1}{2} \gamma_{m}\right.} \\
\quad-A_{m} \beta_{m}^{2} \gamma_{m} \cosh \frac{1}{2} \beta_{m} \sin \frac{1}{2} \gamma_{m}-A_{m} \beta_{m} \gamma_{m}^{2} \sinh \frac{1}{2} \beta_{m} \cos \frac{1}{2} \gamma_{m} \\
-A_{m} \gamma_{m} \beta_{m}^{2} \cosh \frac{1}{2} \beta_{m} \sin \frac{1}{2} \gamma_{m}-A_{m} \gamma_{m}^{2} \beta_{m} \sinh \frac{1}{2} \beta_{m} \cos \frac{1}{2} \gamma_{m} \\
-A_{m} \gamma_{m}^{2} \beta_{m} \sinh \frac{1}{2} \beta_{m} \cos \frac{1}{2} \gamma_{m}+A_{m} \gamma_{m}^{3} \cosh \frac{1}{2} \beta_{m} \sin \frac{1}{2} \gamma_{m} \\
+D_{m} \beta_{m}^{3} \cosh \frac{1}{2} \beta_{m} \sin \frac{1}{2} \gamma_{m}+D_{m} \gamma_{m} \beta_{m}^{2} \sinh \frac{1}{2} \beta_{m} \cos \frac{1}{2} \gamma_{m} \\
+D_{m} \beta_{m}^{2} \gamma_{m} \sinh \frac{1}{2} \beta_{m} \cos \frac{1}{2} \gamma_{m}-D_{m} \gamma_{m}^{2} \beta_{m} \cosh \frac{1}{2} \beta_{m} \sin \frac{1}{2} \gamma_{m} \\
+D_{m} \gamma_{m} \beta_{m}^{2} \sinh \frac{1}{2} \beta_{m} \cos \frac{1}{2} \gamma_{m}-D_{m} \gamma_{m}^{2} \beta_{m} \cosh \frac{1}{2} \beta_{m} \sin \frac{1}{2} \gamma_{m} \\
\left.+D_{m} \gamma_{m}^{2} \beta_{m} \cosh \frac{1}{2} \beta_{m} \sin \frac{1}{2} \gamma_{m}-D_{m} \gamma_{m}^{3} \sinh \frac{1}{2} \beta_{m} \cos \frac{1}{2} \gamma_{m}\right] \sin m \pi \xi \\
\left.+D_{m} \beta_{m} \cosh \frac{1}{2} \beta_{m} \sin \frac{1}{2} \gamma_{m}+D_{m} \gamma_{m} \sinh \frac{1}{2} \beta_{m} \cos \frac{1}{2} \gamma_{m}\right) \sin m \pi \xi=0 \\
-v^{*} \phi^{2}(m \pi)^{2}\left(A_{m} \beta_{m} \sinh \frac{1}{2} \beta_{m} \cos \frac{1}{2} \gamma_{m}-A_{m} \gamma_{m} \cosh \frac{1}{2} \beta_{m} \sin \frac{1}{2} \gamma_{m}\right.
\end{aligned}
$$

These equations can be written as

$$
\begin{aligned}
& \left(A 11 \cdot A_{m}+A 12 \cdot D_{m}\right) \cdot \sin m \pi \xi=0 \\
& \left(A 21 \cdot A_{m}+A 22 \cdot D_{m}\right) \cdot \sin m \pi \xi=0
\end{aligned}
$$

Where,

$$
A 11=\beta_{m}^{2} \cosh \frac{1}{2} \beta_{m} \cos \frac{1}{2} \gamma_{m}-\beta_{m} \gamma_{m} \sinh \frac{1}{2} \beta_{m} \sin \frac{1}{2} \gamma_{m}-\gamma_{m} \beta_{m} \sinh \frac{1}{2} \beta_{m} \sin \frac{1}{2} \gamma_{m}
$$




$$
-\gamma_{m}^{2} \cosh \frac{1}{2} \beta_{m} \cos \frac{1}{2} \gamma_{m}-v_{x y} \phi^{2}(m \pi)^{2} \cosh \frac{1}{2} \beta_{m} \cos \frac{1}{2} \gamma_{m}
$$

and

$$
\begin{aligned}
& A 12=\beta_{m}^{2} \sinh \frac{1}{2} \beta_{m} \sin \frac{1}{2} \gamma_{m}+\gamma_{m} \beta_{m} \cosh \frac{1}{2} \beta_{m} \cos \frac{1}{2} \gamma_{m}+\gamma_{m} \beta_{m} \cosh \frac{1}{2} \beta_{m} \cos \frac{1}{2} \gamma_{m} \\
& -\gamma_{m}^{2} \sinh \frac{1}{2} \beta_{m} \sin \frac{1}{2} \gamma_{m}-v_{x y} \phi^{2}(m \pi)^{2} \sinh \frac{1}{2} \beta_{m} \sin \frac{1}{2} \gamma_{m} \\
& A 21=\beta_{m}^{3} \sinh \frac{1}{2} \beta_{m} \cos \frac{1}{2} \gamma_{m}-\beta_{m}^{2} \gamma_{m} \cosh \frac{1}{2} \beta_{m} \sin \frac{1}{2} \gamma_{m} \\
& -\beta_{m}^{2} \gamma_{m} \cosh \frac{1}{2} \beta_{m} \sin \frac{1}{2} \gamma_{m}-\beta_{m} \gamma_{m}^{2} \sinh \frac{1}{2} \beta_{m} \cos \frac{1}{2} \gamma_{m} \\
& -\gamma_{m} \beta_{m}^{2} \cosh \frac{1}{2} \beta_{m} \sin \frac{1}{2} \gamma_{m}-\gamma_{m}^{2} \beta_{m} \sinh \frac{1}{2} \beta_{m} \cos \frac{1}{2} \gamma_{m} \\
& -\gamma_{m}^{2} \beta_{m} \sinh \frac{1}{2} \beta_{m} \cos \frac{1}{2} \gamma_{m}+\gamma_{m}^{3} \cosh \frac{1}{2} \beta_{m} \sin \frac{1}{2} \gamma_{m} \\
& -v^{*} \phi^{2}(m \pi)^{2}\left(\beta_{m} \sinh \frac{1}{2} \beta_{m} \cos \frac{1}{2} \gamma_{m}-\gamma_{m} \cosh \frac{1}{2} \beta_{m} \sin \frac{1}{2} \gamma_{m}\right) \\
& A 22=\beta_{m}^{3} \cosh \frac{1}{2} \beta_{m} \sin \frac{1}{2} \gamma_{m}+\gamma_{m} \beta_{m}^{2} \sinh \frac{1}{2} \beta_{m} \cos \frac{1}{2} \gamma_{m} \\
& +\beta_{m}^{2} \gamma_{m} \sinh \frac{1}{2} \beta_{m} \cos \frac{1}{2} \gamma_{m}-\gamma_{m}^{2} \beta_{m} \cosh \frac{1}{2} \beta_{m} \sin \frac{1}{2} \gamma_{m} \\
& +\gamma_{m} \beta_{m}^{2} \sinh \frac{1}{2} \beta_{m} \cos \frac{1}{2} \gamma_{m}-\gamma_{m}^{2} \beta_{m} \cosh \frac{1}{2} \beta_{m} \sin \frac{1}{2} \gamma_{m} \\
& -\gamma_{m}^{2} \beta_{m} \cosh \frac{1}{2} \beta_{m} \sin \frac{1}{2} \gamma_{m}-\gamma_{m}^{3} \sinh \frac{1}{2} \beta_{m} \cos \frac{1}{2} \gamma_{m} \\
& -v^{*} \phi^{2}(m \pi)^{2}\left(\beta_{m} \cosh \frac{1}{2} \beta_{m} \sin \frac{1}{2} \gamma_{m}+\gamma_{m} \sinh \frac{1}{2} \beta_{m} \cos \frac{1}{2} \gamma_{m}\right)
\end{aligned}
$$


These equations can be written in a matrix form as

$\left[\begin{array}{ll}A 11 & A 12 \\ A 21 & A 22\end{array}\right]\left[\begin{array}{l}A_{m} \\ D_{m}\end{array}\right]=0$

To satisfy the Equation (5.56), the determinant of the first matrix has to equal to zero.

$\left|\begin{array}{cc}A 11 & A 12 \\ A 21 & A 22\end{array}\right|=0$

This gives us the Eigen-value equation as

$A 11 . A 22-A 12 . A 21=0$

By setting $D_{m}=1$, from Equation (5.55a)

$A_{m}=-\frac{A 12}{A 11}$

By substituting $D_{m}$ and $A_{m}$ in Equation (5.53) and then into Equation (5.20), we get mode shape of the plate as,

$$
w(\xi, \eta)=\left(\sinh \beta_{m} \eta \sin \gamma_{m} \eta-\frac{A 12}{A 11} \cosh \beta_{m} \eta \cos \gamma_{m} \eta\right) \sin m \pi \xi
$$

Anti-symmetrical modes:

For the anti-symmetrical modes the solution of differential Equation (5.19) can be written as

$$
\begin{aligned}
& w(\xi, \eta)=\sum_{m=1}^{k} Y_{m}(\eta) \sin m \pi \xi \\
& w(\xi, \eta)=\left(B_{m} \cosh \beta_{m} \eta \sin \gamma_{m} \eta+C_{m} \sinh \beta_{m} \eta \cos \gamma_{m} \eta\right) \sin m \pi \xi \\
& \frac{\partial w(\xi, \eta)}{\partial \eta}=\left(B_{m} \beta_{m} \sinh \beta_{m} \eta \sin \gamma_{m} \eta+B_{m} \gamma_{m} \cosh \beta_{m} \eta \cos \gamma_{m} \eta\right. \\
& \left.\quad+C_{m} \beta_{m} \cosh \beta_{m} \eta \cos \gamma_{m} \eta-C_{m} \gamma_{m} \sinh \beta_{m} \eta \sin \gamma_{m} \eta\right) \sin m \pi \xi
\end{aligned}
$$




$$
\begin{aligned}
\frac{\partial^{2} w(\xi, \eta)}{\partial \eta^{2}}=( & B_{m} \beta_{m}^{2} \cosh \beta_{m} \eta \sin \gamma_{m} \eta+B_{m} \beta_{m} \gamma_{m} \sinh \beta_{m} \eta \cos \gamma_{m} \eta \\
& -B_{m} \gamma_{m} \beta_{m} \sinh \beta_{m} \eta \cos \gamma_{m} \eta-B_{m} \gamma_{m}^{2} \cosh \beta_{m} \eta \sin \gamma_{m} \eta \\
& +C_{m} \beta_{m}^{2} \sinh \beta_{m} \eta \cos \gamma_{m} \eta-C_{m} \gamma_{m} \beta_{m} \cosh \beta_{m} \eta \sin \gamma_{m} \eta \\
& \left.-C_{m} \gamma_{m} \beta_{m} \cosh \beta_{m} \eta \sin \gamma_{m} \eta-C_{m} \gamma_{m}^{2} \sinh \beta_{m} \eta \cos \gamma_{m} \eta\right) \sin m \pi \xi
\end{aligned}
$$

$$
\begin{aligned}
\frac{\partial^{3} w(\xi, \eta)}{\partial \eta^{3}}=( & B_{m} \beta_{m}^{3} \sinh \beta_{m} \eta \sin \gamma_{m} \eta+B_{m} \beta_{m}^{2} \gamma_{m} \cosh \beta_{m} \eta \cos \gamma_{m} \eta \\
& +B_{m} \beta_{m}^{2} \gamma_{m} \cosh \beta_{m} \eta \cos \gamma_{m} \eta-B_{m} \beta_{m} \gamma_{m}^{2} \sinh \beta_{m} \eta \sin \gamma_{m} \eta \\
& +B_{m} \gamma_{m} \beta_{m}^{2} \cosh \beta_{m} \eta \cos \gamma_{m} \eta-B_{m} \gamma_{m}^{2} \beta_{m} \sinh \beta_{m} \eta \sin \gamma_{m} \eta \\
& -B_{m} \gamma_{m}^{2} \beta_{m} \sinh \beta_{m} \eta \sin \gamma_{m} \eta-B_{m} \gamma_{m}^{3} \cosh \beta_{m} \eta \cos \gamma_{m} \eta \\
& +C_{m} \beta_{m}^{3} \cosh \beta_{m} \eta \cos \gamma_{m} \eta-C_{m} \gamma_{m} \beta_{m}^{2} \sinh \beta_{m} \eta \sin \gamma_{m} \eta \\
& -C_{m} \beta_{m}^{2} \gamma_{m} \sinh \beta_{m} \eta \sin \gamma_{m} \eta-C_{m} \gamma_{m}^{2} \beta_{m} \cosh \beta_{m} \eta \cos \gamma_{m} \eta \\
& -C_{m} \gamma_{m} \beta_{m}^{2} \sinh \beta_{m} \eta \sin \gamma_{m} \eta-C_{m} \gamma_{m}^{2} \beta_{m} \cosh \beta_{m} \eta \cos \gamma_{m} \eta \\
& \left.-C_{m} \gamma_{m}^{2} \beta_{m} \cosh \beta_{m} \eta \cos \gamma_{m} \eta+C_{m} \gamma_{m}^{3} \sinh \beta_{m} \eta \sin \gamma_{m} \eta\right) \sin m \pi \xi
\end{aligned}
$$

$\frac{\partial w(\xi, \eta)}{\partial \xi}=m \pi\left(B_{m} \cosh \beta_{m} \eta \cos \gamma_{m} \eta+C_{m} \sinh \beta_{m} \eta \sin \gamma_{m} \eta\right) \cos m \pi \xi$

$\frac{\partial^{2} w(\xi, \eta)}{\partial \xi^{2}}=-(m \pi)^{2}\left(B_{m} \cosh \beta_{m} \eta \cos \gamma_{m} \eta+C_{m} \sinh \beta_{m} \eta \sin \gamma_{m} \eta\right) \sin m \pi \xi$

$$
\begin{aligned}
\frac{\partial^{3} w(\xi, \eta)}{\partial \eta \partial \xi^{2}}=-( & m \pi)^{2}\left(B_{m} \beta_{m} \sinh \beta_{m} \eta \sin \gamma_{m} \eta+B_{m} \gamma_{m} \cosh \beta_{m} \eta \cos \gamma_{m} \eta\right. \\
& \left.+C_{m} \beta_{m} \cosh \beta_{m} \eta \cos \gamma_{m} \eta-C_{m} \gamma_{m} \sinh \beta_{m} \eta \sin \gamma_{m} \eta\right) \sin m \pi \xi
\end{aligned}
$$

The boundary conditions of Equation (5.29) become 


$$
\begin{aligned}
& {\left[B_{m} \beta_{m}^{2} \cosh \frac{1}{2} \beta_{m} \sin \frac{1}{2} \gamma_{m}+B_{m} \beta_{m} \gamma_{m} \sinh \frac{1}{2} \beta_{m} \cos \frac{1}{2} \gamma_{m}\right.} \\
& -B_{m} \gamma_{m} \beta_{m} \sinh \frac{1}{2} \beta_{m} \cos \frac{1}{2} \gamma_{m}-B_{m} \gamma_{m}^{2} \cosh \frac{1}{2} \beta_{m} \sin \frac{1}{2} \gamma_{m} \\
& +C_{m} \beta_{m}^{2} \sinh \frac{1}{2} \beta_{m} \cos \frac{1}{2} \gamma_{m}-C_{m} \gamma_{m} \beta_{m} \cosh \frac{1}{2} \beta_{m} \sin \frac{1}{2} \gamma_{m} \\
& \left.-C_{m} \gamma_{m} \beta_{m} \cosh \frac{1}{2} \beta_{m} \sin \frac{1}{2} \gamma_{m}-C_{m} \gamma_{m}^{2} \sinh \frac{1}{2} \beta_{m} \cos \frac{1}{2} \gamma_{m}\right] \sin m \pi \xi \\
& -v_{x y} \phi^{2}(m \pi)^{2}\left(B_{m} \cosh \frac{1}{2} \beta_{m} \cos \frac{1}{2} \gamma_{m}+C_{m} \sinh \frac{1}{2} \beta_{m} \sin \frac{1}{2} \gamma_{m}\right) \sin m \pi \xi=0
\end{aligned}
$$

and,

$$
\begin{aligned}
& {\left[B_{m} \beta_{m}^{3} \sinh \frac{1}{2} \beta_{m} \sin \frac{1}{2} \gamma_{m}+B_{m} \beta_{m}^{2} \gamma_{m} \cosh \frac{1}{2} \beta_{m} \cos \frac{1}{2} \gamma_{m}\right.} \\
& +B_{m} \beta_{m}^{2} \gamma_{m} \cosh \frac{1}{2} \beta_{m} \cos \frac{1}{2} \gamma_{m}-B_{m} \beta_{m} \gamma_{m}^{2} \sinh \frac{1}{2} \beta_{m} \sin \frac{1}{2} \gamma_{m} \\
& +B_{m} \gamma_{m} \beta_{m}^{2} \cosh \frac{1}{2} \beta_{m} \cos \frac{1}{2} \gamma_{m}-B_{m} \gamma_{m}^{2} \beta_{m} \sinh \frac{1}{2} \beta_{m} \sin \frac{1}{2} \gamma_{m} \\
& -B_{m} \gamma_{m}^{2} \beta_{m} \sinh \frac{1}{2} \beta_{m} \sin \frac{1}{2} \gamma_{m}-B_{m} \gamma_{m}^{3} \cosh \frac{1}{2} \beta_{m} \cos \frac{1}{2} \gamma_{m} \\
& +C_{m} \beta_{m}^{3} \cosh \frac{1}{2} \beta_{m} \cos \frac{1}{2} \gamma_{m}-C_{m} \gamma_{m} \beta_{m}^{2} \sinh \frac{1}{2} \beta_{m} \sin \frac{1}{2} \gamma_{m} \\
& -C_{m} \beta_{m}^{2} \gamma_{m} \sinh \frac{1}{2} \beta_{m} \sin \frac{1}{2} \gamma_{m}-C_{m} \gamma_{m}^{2} \beta_{m} \cosh \frac{1}{2} \beta_{m} \cos \frac{1}{2} \gamma_{m} \\
& -C_{m} \gamma_{m} \beta_{m}^{2} \sinh \frac{1}{2} \beta_{m} \sin \frac{1}{2} \gamma_{m}-C_{m} \gamma_{m}^{2} \beta_{m} \cosh \frac{1}{2} \beta_{m} \cos \frac{1}{2} \gamma_{m} \\
& \left.-C_{m} \gamma_{m}^{2} \beta_{m} \cosh \frac{1}{2} \beta_{m} \cos \frac{1}{2} \gamma_{m}+C_{m} \gamma_{m}^{3} \sinh \frac{1}{2} \beta_{m} \sin \frac{1}{2} \gamma_{m}\right] \sin m \pi \xi \\
& 2
\end{aligned}
$$

61 
$\left.+C_{m} \beta_{m} \cosh \frac{1}{2} \beta_{m} \cos \frac{1}{2} \gamma_{m}-C_{m} \gamma_{m} \sinh \frac{1}{2} \beta_{m} \sin \frac{1}{2} \gamma_{m}\right) \sin m \pi \xi=0$

These equations can be written as

$\left(A 11 \cdot B_{m}+A 12 \cdot C_{m}\right) \cdot \sin m \pi \xi=0$

$\left(A 21 \cdot B_{m}+A 22 \cdot C_{m}\right) \cdot \sin m \pi \xi=0$

Where,

$$
\begin{aligned}
& A 11=\beta_{m}^{2} \cosh \frac{1}{2} \beta_{m} \sin \frac{1}{2} \gamma_{m}+\beta_{m} \gamma_{m} \sinh \frac{1}{2} \beta_{m} \cos \frac{1}{2} \gamma_{m}-\gamma_{m} \beta_{m} \sinh \frac{1}{2} \beta_{m} \cos \frac{1}{2} \gamma_{m} \\
& -\gamma_{m}^{2} \cosh \frac{1}{2} \beta_{m} \sin \frac{1}{2} \gamma_{m}-v_{x y} \phi^{2}(m \pi)^{2} \cosh \frac{1}{2} \beta_{m} \cos \frac{1}{2} \gamma_{m} \\
& A 12=\beta_{m}^{2} \sinh \frac{1}{2} \beta_{m} \cos \frac{1}{2} \gamma_{m}-\gamma_{m} \beta_{m} \cosh \frac{1}{2} \beta_{m} \sin \frac{1}{2} \gamma_{m}-\gamma_{m} \beta_{m} \cosh \frac{1}{2} \beta_{m} \sin \frac{1}{2} \gamma_{m} \\
& -\gamma_{m}^{2} \sinh \frac{1}{2} \beta_{m} \cos \frac{1}{2} \gamma_{m}-v_{x y} \phi^{2}(m \pi)^{2} \sinh \frac{1}{2} \beta_{m} \sin \frac{1}{2} \gamma_{m} \\
& A 21=\beta_{m}^{3} \sinh \frac{1}{2} \beta_{m} \sin \frac{1}{2} \gamma_{m}+\beta_{m}^{2} \gamma_{m} \cosh \frac{1}{2} \beta_{m} \cos \frac{1}{2} \gamma_{m} \\
& +\beta_{m}^{2} \gamma_{m} \cosh \frac{1}{2} \beta_{m} \cos \frac{1}{2} \gamma_{m}-\beta_{m} \gamma_{m}^{2} \sinh \frac{1}{2} \beta_{m} \sin \frac{1}{2} \gamma_{m} \\
& +\gamma_{m} \beta_{m}^{2} \cosh \frac{1}{2} \beta_{m} \cos \frac{1}{2} \gamma_{m}-\gamma_{m}^{2} \beta_{m} \sinh \frac{1}{2} \beta_{m} \sin \frac{1}{2} \gamma_{m} \\
& -\gamma_{m}^{2} \beta_{m} \sinh \frac{1}{2} \beta_{m} \sin \frac{1}{2} \gamma_{m}-\gamma_{m}^{3} \cosh \frac{1}{2} \beta_{m} \cos \frac{1}{2} \gamma_{m} \\
& -v^{*} \phi^{2}(m \pi)^{2}\left(\beta_{m} \sinh \frac{1}{2} \beta_{m} \sin \frac{1}{2} \gamma_{m}+\gamma_{m} \cosh \frac{1}{2} \beta_{m} \cos \frac{1}{2} \gamma_{m}\right) \\
& A 22=\beta_{m}^{3} \cosh \frac{1}{2} \beta_{m} \cos \frac{1}{2} \gamma_{m}-\gamma_{m} \beta_{m}^{2} \sinh \frac{1}{2} \beta_{m} \sin \frac{1}{2} \gamma_{m} \\
& -\beta_{m}^{2} \gamma_{m} \sinh \frac{1}{2} \beta_{m} \sin \frac{1}{2} \gamma_{m}-\gamma_{m}^{2} \beta_{m} \cosh \frac{1}{2} \beta_{m} \cos \frac{1}{2} \gamma_{m}
\end{aligned}
$$




$$
\begin{aligned}
& -\gamma_{m} \beta_{m}^{2} \sinh \frac{1}{2} \beta_{m} \sin \frac{1}{2} \gamma_{m}-\gamma_{m}^{2} \beta_{m} \cosh \frac{1}{2} \beta_{m} \cos \frac{1}{2} \gamma_{m} \\
& -\gamma_{m}^{2} \beta_{m} \cosh \frac{1}{2} \beta_{m} \cos \frac{1}{2} \gamma_{m}+\gamma_{m}^{3} \sinh \frac{1}{2} \beta_{m} \sin \frac{1}{2} \gamma_{m} \\
& -v^{*} \phi^{2}(m \pi)^{2}\left(\beta_{m} \cosh \frac{1}{2} \beta_{m} \cos \frac{1}{2} \gamma_{m}-\gamma_{m} \sinh \frac{1}{2} \beta_{m} \sin \frac{1}{2} \gamma_{m}\right)
\end{aligned}
$$

These equations can be written in a matrix form as,

$\left[\begin{array}{ll}A 11 & A 12 \\ A 21 & A 22\end{array}\right]\left[\begin{array}{l}B_{m} \\ C_{m}\end{array}\right]=0$

To satisfy the Equation (5.61), the determinant of the first matrix has to equal to zero.

$\left|\begin{array}{ll}A 11 & A 12 \\ A 21 & A 22\end{array}\right|=0$

This gives us the Eigen-value equation as

$A 11 . A 22-A 12 \cdot A 21=0$

By setting $C_{m}=1$, from Equation (5.60a)

$B_{m}=-\frac{A 12}{A 11}$

By substituting $C_{m}$ and $B_{m}$ in Equation (5.54) and then into Equation (5.20), we get mode shape of the plate as,

$$
w(\xi, \eta)=\left(\sinh \beta_{m} \eta \cos \gamma_{m} \eta-\frac{A 12}{A 11} \cosh \beta_{m} \eta \sin \gamma_{m} \eta\right) \sin m \pi \xi
$$

As seen earlier, the modulus of rigidities such as flexural rigidity and torsional rigidity $\left(D_{x}, D_{y}, H\right)$ tell us the behavior of the plate. Depending upon the values of $C_{1}=\frac{H}{D_{y}}(m \pi)^{2}$ and $C_{2}=\frac{D_{x}}{D_{y}}(m \pi)^{4}$, we have two different categories. 
Category I:

If $C_{2}-C_{1}^{2}<\lambda^{4}$

This category represents the plates which are stronger in torsion than in flexure and is subdivided into two subcategories as,

Category I(a):

$$
\text { If } C_{1}>\sqrt{C_{1}^{2}-C_{2}+\lambda^{4}} \text { and, }
$$

Category I(b):

$$
\text { If } C_{1}<\sqrt{C_{1}^{2}-C_{2}+\lambda^{4}}
$$

Category II:

$$
\text { If } C_{2}-C_{1}^{2}>\lambda^{4}
$$

This category represents the plates which are stronger in flexure than in torsion. Depending upon the natural frequency of the plate $\lambda^{4}$, the plate in consideration either falls into category I(a), I(b) or category II for each mode. Only the first four natural frequencies $(m=1,2: n=1,2)$ are considered in this study. The Eigen-value Equations (5.33), (5.38), (5.45), (5.50), (5.58), and (5.62) are used to calculate the Eigen-values of the orthotropic plates.

A MATLAB program is written to solve these equations by trial and error method. To verify the results from this program, a sample isotropic plate with two opposite edges simply supported and the other two free is considered and the eigenvalues are compared with the eigen-values presented by Gorman D. J. [14] as shown in Table 6. An isotropic plate with the aspect ratio of $\frac{b}{a}=1$ and poisons ratio of 0.333 is considered for the comparison. 
Table 6: Comparison of the first four eigen-values with the eigen-values derived by Gorman D. J. [14].

\begin{tabular}{ccc}
\hline Modes & Gorman D. J. & Current study \\
\hline$(1,1)$ & 9.568 & 9.565 \\
$(1,2)$ & 15.88 & 15.885 \\
$(2,1)$ & 38.79 & 38.795 \\
$(2,2)$ & 46.33 & 46.335 \\
\hline
\end{tabular}

From Table 6, we can see that the eigen-values calculated by a program in MATLAB are in very good agreement with the eigen-values derived by Gorman D. J. [14].

To verify the accuracy of the program for orthotropic plates, an orthotropic plate (Two edges simply supported and the other two free) used by Jayaraman G. et. al. [6] with the aspect ratio of 1 (i.e. $\frac{b}{a}=1$ ) and poisons ratio of 0.3 is solved for different rigidity ratios, and the first four Eigen-values are compared with the frequency parameters given by Jayaraman G. et. al. [6]. We can see from Table 7 that the Eigenvalues calculated from a MATLAB program are well matched with the frequency parameter given by Jayaraman G. et. al. [6].

Table 8 shows us the mode shape categorization of orthotropic plates for different values of $C_{1}=\frac{H}{D_{y}}(m \pi)^{2}$ and $C_{2}=\frac{D_{x}}{D_{y}}(m \pi)^{4}$ for the modes with $m=1$, that means for the modes $(1,1)$ and $(1,2)$. 
Table 7: Comparison of the first four eigen-values with the frequency parameters derived by Jayaraman et. al. [6].

\begin{tabular}{cccccccc}
\hline & & \multicolumn{7}{c}{ H/Dy } \\
\cline { 3 - 8 } Dx/Dy Modes & \multicolumn{2}{c}{0.5} & \multicolumn{2}{c}{1} & \\
\cline { 3 - 8 } & & $\begin{array}{c}\text { Jayaraman } \\
\text { et. al. }\end{array}$ & $\begin{array}{c}\text { Current } \\
\text { study }\end{array}$ & $\begin{array}{c}\text { Jayaraman } \\
\text { et. al. }\end{array}$ & $\begin{array}{c}\text { Current } \\
\text { study }\end{array}$ & $\begin{array}{c}\text { Jayaraman } \\
\text { et. al. }\end{array}$ & $\begin{array}{c}\text { Current } \\
\text { study }\end{array}$ \\
\hline \multirow{4}{*}{0.5} & $(1,1)$ & 6.4705 & 6.45 & 6.6377 & 6.65 & 6.7529 & 6.75 \\
& $(1,2)$ & 9.5537 & 9.55 & 14.5474 & 14.55 & 20.8297 & 20.85 \\
& $(2,1)$ & 26.4882 & 26.45 & 27.1557 & 27.15 & 27.4505 & 27.45 \\
& $(2,2)$ & 29.9614 & 29.95 & 37.4857 & 37.45 & 47.6178 & 47.65 \\
\hline \multirow{4}{*}{1} & $(1,1)$ & 9.5169 & 9.55 & 9.6314 & 9.65 & 9.7111 & 9.75 \\
& $(1,2)$ & 11.8312 & 11.85 & 16.1348 & 16.15 & 21.9677 & 21.95 \\
& $(2,1)$ & 38.4824 & 38.45 & 38.9449 & 38.95 & 39.151 & 39.15 \\
& $(2,2)$ & 40.9506 & 40.95 & 46.7381 & 46.75 & 55.1972 & 55.15 \\
\hline \multirow{4}{*}{2} & $(1,1)$ & 13.7106 & 13.75 & 13.7903 & 13.75 & 13.8461 & 13.85 \\
& $(1,2)$ & 15.4074 & 15.45 & 18.9140 & 18.95 & 24.0830 & 24.05 \\
& $(2,1)$ & 55.1311 & 55.15 & 55.4550 & 55.45 & 55.5998 & 55.55 \\
& $(2,2)$ & 56.8814 & 56.85 & 61.1800 & 61.15 & 67.8621 & 67.85 \\
\hline
\end{tabular}


Table 8: Categorization of orthotropic plates in accordance with the rigidity ratios.

\begin{tabular}{llllll}
\hline$\frac{D x}{D y}$ & & $H / D y=0.25$ & $H / D y=0.5$ & $H / D y=1$ & $H / D y=2$ \\
\hline $1 / 2$ & II & $0<\lambda^{2} \leq 6.528$ & $0<\lambda^{2} \leq 4.934$ & - & - \\
& I(a) & $6.528<\lambda^{2} \leq 6.978$ & $4.934<\lambda^{2} \leq 6.978$ & $0<\lambda^{2} \leq 6.978$ & $0<\lambda^{2} \leq 6.978$ \\
& I(b) & $6.978<\lambda^{2}$ & $6.978<\lambda^{2}$ & $6.978<\lambda^{2}$ & $6.978<\lambda^{2}$ \\
\hline 1 & II & $0<\lambda^{2} \leq 9.556$ & $0<\lambda^{2} \leq 8.547$ & - & - \\
& I(a) & $9.556<\lambda^{2} \leq 9.869$ & $8.547<\lambda^{2} \leq 9.869$ & $0<\lambda^{2} \leq 9.869$ & $0<\lambda^{2} \leq 9.869$ \\
& I(b) & $9.869<\lambda^{2}$ & $9.869<\lambda^{2}$ & $9.869<\lambda^{2}$ & $9.869<\lambda^{2}$ \\
\hline 2 & II & $0<\lambda^{2} \leq 13.737$ & $0<\lambda^{2} \leq 13.056$ & $0<\lambda^{2} \leq 9.8696$ & - \\
& I(a) & $13.737<\lambda^{2} \leq 13.957$ & $13.056<\lambda^{2} \leq 13.957$ & $9.8696<\lambda^{2} \leq 13.957$ & $0<\lambda^{2} \leq 13.957$ \\
& I(b) & $13.957<\lambda^{2}$ & $13.957<\lambda^{2}$ & $13.957<\lambda^{2}$ & $13.957<\lambda^{2}$ \\
\hline 3 & II & $0<\lambda^{2} \leq 16.915$ & $0<\lambda^{2} \leq 16.366$ & $0<\lambda^{2} \leq 13.957$ & - \\
& I(a) & $16.915<\lambda^{2} \leq 17.094$ & $16.366<\lambda^{2} \leq 17.094$ & $13.957<\lambda^{2} \leq 17.094$ & $0<\lambda^{2} \leq 17.094$ \\
& I(b) & $17.094<\lambda^{2}$ & $17.094<\lambda^{2}$ & $17.094<\lambda^{2}$ & $17.094<\lambda^{2}$ \\
\hline 4 & II & $0<\lambda^{2} \leq 19.584$ & $0<\lambda^{2} \leq 19.112$ & $0<\lambda^{2} \leq 19.094$ & - \\
& I(a) & $19.584<\lambda^{2} \leq 19.739$ & $19.112<\lambda^{2} \leq 19.739$ & $19.094<\lambda^{2} \leq 19.739$ & $0<\lambda^{2} \leq 19.739$ \\
& I(b) & $19.739<\lambda^{2}$ & $19.739<\lambda^{2}$ & $19.739<\lambda^{2}$ & $19.739<\lambda^{2}$ \\
\hline
\end{tabular}


Table 8 contd: Categorization of orthotropic plates in accordance with the rigidity ratios.

\begin{tabular}{llllll}
\hline$\frac{D x}{D y}$ & & $H / D y=0.25$ & $H / D y=0.5$ & $H / D y=1$ & $H / D y=2$ \\
\hline 5 & II & $0<\lambda^{2} \leq 21.930$ & $0<\lambda^{2} \leq 21.510$ & $0<\lambda^{2} \leq 19.739$ & $0<\lambda^{2} \leq 9.869$ \\
& I(a) & $21.930<\lambda^{2} \leq 22.069$ & $21.510<\lambda^{2} \leq 22.069$ & $19.739<\lambda^{2} \leq 22.069$ & $9.869<\lambda^{2} \leq 22.069$ \\
& I(b) & $22.069<\lambda^{2}$ & $22.069<\lambda^{2}$ & $22.069<\lambda^{2}$ & $22.069<\lambda^{2}$ \\
\hline 6 & II & $0<\lambda^{2} \leq 24.049$ & $0<\lambda^{2} \leq 23.666$ & $0<\lambda^{2} \leq 22.069$ & $0<\lambda^{2} \leq 13.957$ \\
& I(a) & $24.049<\lambda^{2} \leq 24.175$ & $23.666<\lambda^{2} \leq 24.175$ & $22.069<\lambda^{2} \leq 24.175$ & $13.957<\lambda^{2} \leq 24.175$ \\
& I(b) & $24.175<\lambda^{2}$ & $24.175<\lambda^{2}$ & $24.175<\lambda^{2}$ & $24.175<\lambda^{2}$ \\
\hline 7 & II & $0<\lambda^{2} \leq 25.995$ & $0<\lambda^{2} \leq 25.641$ & $0<\lambda^{2} \leq 24.175$ & $0<\lambda^{2} \leq 17.094$ \\
& I(a) & $25.995<\lambda^{2} \leq 26.112$ & $25.641<\lambda^{2} \leq 26.112$ & $24.175<\lambda^{2} \leq 26.112$ & $17.094<\lambda^{2} \leq 26.112$ \\
& I(b) & $26.112<\lambda^{2}$ & $26.112<\lambda^{2}$ & $26.112<\lambda^{2}$ & $26.112<\lambda^{2}$ \\
\hline 8 & II & $0<\lambda^{2} \leq 27.806$ & $0<\lambda^{2} \leq 27.475$ & $0<\lambda^{2} \leq 26.112$ & $0<\lambda^{2} \leq 19.739$ \\
& I(a) & $27.806<\lambda^{2} \leq 27.915$ & $27.475<\lambda^{2} \leq 27.915$ & $26.112<\lambda^{2} \leq 27.915$ & $19.739<\lambda^{2} \leq 27.915$ \\
& I(b) & $27.915<\lambda^{2}$ & $27.915<\lambda^{2}$ & $27.915<\lambda^{2}$ & $27.915<\lambda^{2}$ \\
\hline 9 & II & $0<\lambda^{2} \leq 29.505$ & $0<\lambda^{2} \leq 29.194$ & $0<\lambda^{2} \leq 27.915$ & $0<\lambda^{2} \leq 22.069$ \\
& I(a) & $29.505<\lambda^{2} \leq 29.608$ & $29.194<\lambda^{2} \leq 29.608$ & $27.915<\lambda^{2} \leq 29.608$ & $22.069<\lambda^{2} \leq 29.608$ \\
& I(b) & $29.608<\lambda^{2}$ & $29.608<\lambda^{2}$ & $29.608<\lambda^{2}$ & $29.608<\lambda^{2}$ \\
\hline
\end{tabular}


To study the mode shape categorization of orthotropic plates according to the rigidity ratios, a sample orthotropic plate is considered as shown is Figure 13.

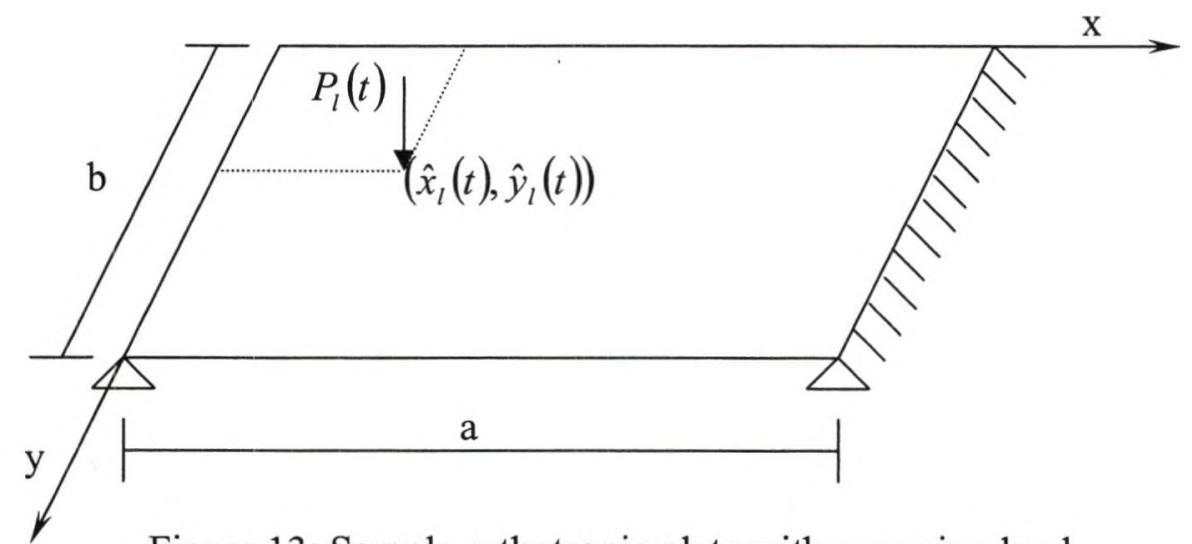

Figure 13: Sample orthotropic plate with a moving load

In this investigation, the sample data used for the analysis is

$$
\begin{array}{ll}
a=36 f t & h=0.5 f t \\
b=18 f t & v_{x y}=0.333 \\
\phi=b / a=0.5 &
\end{array}
$$

Table 9 shows the first four Eigen-values of orthotropic plates for different rigidity ratios.

These Eigen-values are compared with the categorization limits given in Table 8. In the Table 10, the categories are defined for an orthotropic plate with different rigidity ratios. For all the plates with any ratio of flexural rigidity in $\mathrm{X}$ and $\mathrm{Y}$ direction considered in this investigation, the plates with the torsional rigidity ratio, $\frac{H}{D_{y}}$ of 0.25 only falls in the category II for modes $(1,1)$ and $(2,1)$. All the other plates with higher torsional rigidity ratios fall under category I-a for modes $(1,1)$ and $(2,1)$. All the plates studied here fall in the category I-b for modes $(1,2)$ and $(2,2)$. 
Table 9: First four eigen-values of an orthotropic plate for different rigidity ratios.

\begin{tabular}{|c|c|c|c|c|c|}
\hline \multirow{2}{*}{ Dx/Dy } & \multirow{2}{*}{ Mode } & \multicolumn{4}{|c|}{ H/Dy } \\
\hline & & 0.25 & 0.5 & 1 & 2 \\
\hline \multirow{4}{*}{0.5} & $(1,1)$ & 6.15 & 6.25 & 6.35 & 6.45 \\
\hline & $(1,2)$ & 247.85 & 14.15 & 26.05 & 40.05 \\
\hline & $(2,1)$ & 24.25 & 25.25 & 26.15 & 26.75 \\
\hline & $(2,2)$ & 252.35 & 36.05 & 57.05 & 82.75 \\
\hline \multirow{4}{*}{1} & $(1,1)$ & 9.25 & 9.35 & 9.45 & 9.55 \\
\hline & $(1,2)$ & 247.95 & 15.75 & 26.95 & 40.65 \\
\hline & $(2,1)$ & 36.95 & 37.65 & 38.25 & 38.65 \\
\hline & $(2,2)$ & 253.85 & 45.65 & 63.55 & 87.35 \\
\hline \multirow{4}{*}{2} & $(1,1)$ & 13.55 & 13.55 & 13.65 & 13.75 \\
\hline & $(1,2)$ & 248.15 & 18.65 & 28.65 & 41.85 \\
\hline & $(2,1)$ & 54.05 & 54.55 & 54.95 & 55.25 \\
\hline & $(2,2)$ & 256.95 & 60.35 & 74.75 & 95.85 \\
\hline \multirow{4}{*}{3} & $(1,1)$ & 16.75 & 16.85 & 16.85 & 16.85 \\
\hline & $(1,2)$ & 248.35 & 21.05 & 30.35 & 42.95 \\
\hline & $(2,1)$ & 66.95 & 67.35 & 67.65 & 67.95 \\
\hline & $(2,2)$ & 259.95 & 72.15 & 84.55 & 103.65 \\
\hline \multirow{4}{*}{4} & $(1,1)$ & 19.45 & 19.45 & 19.55 & 19.55 \\
\hline & $(1,2)$ & 248.55 & 23.25 & 31.95 & 44.15 \\
\hline & $(2,1)$ & 77.75 & 78.05 & 78.35 & 78.55 \\
\hline & $(2,2)$ & 262.95 & 82.25 & 93.35 & 110.95 \\
\hline \multirow{4}{*}{5} & $(1,1)$ & 21.85 & 21.85 & 21.85 & 21.95 \\
\hline & $(1,2)$ & 248.75 & 25.25 & 33.45 & 45.15 \\
\hline & $(2,1)$ & 87.15 & 87.45 & 87.75 & 87.95 \\
\hline & $(2,2)$ & 265.85 & 91.15 & 101.35 & 117.75 \\
\hline \multirow{4}{*}{6} & $(1,1)$ & 23.95 & 23.95 & 24.05 & 24.05 \\
\hline & $(1,2)$ & 248.95 & 27.15 & 34.85 & 46.25 \\
\hline & $(2,1)$ & 95.65 & 95.95 & 96.25 & 96.35 \\
\hline & $(2,2)$ & 268.75 & 99.35 & 108.75 & 124.15 \\
\hline \multirow{4}{*}{7} & $(1,1)$ & 25.85 & 25.95 & 25.95 & 25.95 \\
\hline & $(1,2)$ & 249.05 & 28.85 & 36.25 & 47.25 \\
\hline & $(2,1)$ & 103.55 & 103.75 & 104.05 & 104.15 \\
\hline & $(2,2)$ & 271.65 & 106.95 & 115.75 & 130.25 \\
\hline \multirow{4}{*}{8} & $(1,1)$ & 27.75 & 27.75 & 27.75 & 27.75 \\
\hline & $(1,2)$ & 249.25 & 30.55 & 37.55 & 48.35 \\
\hline & $(2,1)$ & 110.75 & 111.05 & 111.25 & 111.35 \\
\hline & $(2,2)$ & 274.55 & 113.95 & 122.25 & 136.15 \\
\hline \multirow{4}{*}{9} & $(1,1)$ & 29.45 & 29.45 & 29.45 & 29.45 \\
\hline & $(1,2)$ & 249.45 & 32.05 & 38.75 & 49.35 \\
\hline & $(2,1)$ & 118.05 & 117.85 & 118.05 & 118.15 \\
\hline & $(2,2)$ & 277.35 & 120.65 & 128.45 & 141.75 \\
\hline
\end{tabular}


Table 10: Categories of orthotropic plates with different rigidity ratios for the first four mode shapes.

\begin{tabular}{|c|c|c|c|c|c|}
\hline \multirow{2}{*}{ Dx/Dy } & \multirow{2}{*}{ Mode } & \multicolumn{4}{|c|}{ H/Dy } \\
\hline & & 0.25 & 0.5 & 1 & 2 \\
\hline \multirow{4}{*}{0.5} & $(1,1)$ & II & $\mathrm{I}-\mathrm{a}$ & $\mathrm{I}-\mathrm{a}$ & I-a \\
\hline & $(1,2)$ & I-b & I-b & $1-b$ & $1-b$ \\
\hline & $(2,1)$ & $\|$ & $\mathrm{I}-\mathrm{a}$ & $\mathrm{I}-\mathrm{a}$ & $\mathrm{I}-\mathrm{a}$ \\
\hline & $(2,2)$ & $\mathrm{I}-\mathrm{b}$ & I-b & $1-b$ & $1-b$ \\
\hline \multirow{4}{*}{1} & $(1,1)$ & $\|$ & $\mathrm{I}-\mathrm{a}$ & $\mathrm{I}-\mathrm{a}$ & I-a \\
\hline & $(1,2)$ & I-b & I-b & $I-b$ & $\mathrm{I}-\mathrm{b}$ \\
\hline & $(2,1)$ & II & $\mathrm{I}-\mathrm{a}$ & $\mathrm{I}-\mathrm{a}$ & I-a \\
\hline & $(2,2)$ & $1-b$ & $\mathrm{I}-\mathrm{b}$ & $\mathrm{I}-\mathrm{b}$ & $1-b$ \\
\hline \multirow{4}{*}{2} & $(1,1)$ & II & $\mathrm{I}-\mathrm{a}$ & $\mathrm{I}-\mathrm{a}$ & I-a \\
\hline & $(1,2)$ & I-b & $I-b$ & $I-b$ & $1-b$ \\
\hline & $(2,1)$ & $\|$ & $\mathrm{I}-\mathrm{a}$ & $\mathrm{I}-\mathrm{a}$ & $\mathrm{I}-\mathrm{a}$ \\
\hline & $(2,2)$ & $\mathrm{I}-\mathrm{b}$ & $1-b$ & $1-b$ & $1-b$ \\
\hline \multirow{4}{*}{3} & $(1,1)$ & II & $\mathrm{I}-\mathrm{a}$ & $\mathrm{I}-\mathrm{a}$ & I-a \\
\hline & $(1,2)$ & I-b & I-b & $I-b$ & $1-b$ \\
\hline & $(2,1)$ & II & I-a & $\mathrm{I}-\mathrm{a}$ & $1-a$ \\
\hline & $(2,2)$ & $1-b$ & $1-b$ & $\mathrm{I}-\mathrm{b}$ & $1-b$ \\
\hline \multirow{4}{*}{4} & $(1,1)$ & II & I-a & $\mathrm{I}-\mathrm{a}$ & $1-a$ \\
\hline & $(1,2)$ & $I-b$ & $1-b$ & $I-b$ & I-b \\
\hline & $(2,1)$ & II & I-a & $\mathrm{I}-\mathrm{a}$ & $\mathrm{I}-\mathrm{a}$ \\
\hline & $(2,2)$ & $1-b$ & $1-b$ & $1-b$ & I-b \\
\hline \multirow{4}{*}{5} & $(1,1)$ & II & $\mathrm{I}-\mathrm{a}$ & $\mathrm{I}-\mathrm{a}$ & $\mathrm{I}-\mathrm{a}$ \\
\hline & $(1,2)$ & $I-b$ & $1-b$ & $I-b$ & I-b \\
\hline & $(2,1)$ & II & $1-a$ & $\mathrm{I}-\mathrm{a}$ & I-a \\
\hline & $(2,2)$ & $\mathrm{I}-\mathrm{b}$ & I-b & $1-b$ & I-b \\
\hline \multirow{4}{*}{6} & $(1,1)$ & II & I-a & $\mathrm{I}-\mathrm{a}$ & $\mathrm{I}-\mathrm{a}$ \\
\hline & $(1,2)$ & $I-b$ & $1-b$ & $1-b$ & I-b \\
\hline & $(2,1)$ & II & $\mathrm{I}-\mathrm{a}$ & I-a & $\mathrm{I}-\mathrm{a}$ \\
\hline & $(2,2)$ & $\mathrm{I}-\mathrm{b}$ & $1-b$ & $1-b$ & I-b \\
\hline \multirow{4}{*}{7} & $(1,1)$ & II & I-a & $\mathrm{I}-\mathrm{a}$ & $\mathrm{I}-\mathrm{a}$ \\
\hline & $(1,2)$ & I-b & $1-b$ & I-b & $1-b$ \\
\hline & $(2,1)$ & II & $1-a$ & I-a & I-a \\
\hline & $(2,2)$ & $1-b$ & $1-b$ & $1-b$ & I-b \\
\hline \multirow{4}{*}{8} & $(1,1)$ & II & I-a & I-a & I-a \\
\hline & $(1,2)$ & $I-b$ & I-b & $1-b$ & $I-b$ \\
\hline & $(2,1)$ & II & $\mathrm{I}-\mathrm{a}$ & I-a & I-a \\
\hline & $(2,2)$ & $1-b$ & $\mathrm{I}-\mathrm{b}$ & I-b & $1-b$ \\
\hline \multirow{4}{*}{9} & $(1,1)$ & II & $\mathrm{I}-\mathrm{a}$ & $1-a$ & I-a \\
\hline & $(1,2)$ & $1-b$ & I-b & $1-b$ & I-b \\
\hline & $(2,1)$ & $\|$ & $\mathrm{I}-\mathrm{a}$ & $\mathrm{I}-\mathrm{a}$ & I-a \\
\hline & $(2,2)$ & $1-b$ & I-b & $1-b$ & $1-b$ \\
\hline
\end{tabular}




\section{V-c: Analysis of Orthotropic Plates under Moving Loads}

Natural mode shapes $w_{m n}(x, y)$ calculated for each category as explained in the previous section are substituted into the equation of motion given in Equation (5.13) to get the mass matrix, damping matrix, stiffness matrix, and the force matrix. Two MATLAB programs are written to calculate the response in terms of vertical displacements of an orthotropic plate by considering two different possibilities as per the Table 10. First program gives the response of the plates which fall into category-Ia for modes $(1,1)$ and $(2,1)$, and fall into category-Ib for modes $(1,2)$ and $(2,2)$. Second program gives the response in terms of vertical displacements of the plates which fall into category-II for modes $(1,1)$ and $(2,1)$, and fall into category-Ib for modes $(1,2)$ and $(2,2)$. Depending on in which category the given orthotropic plate falls in, the respective natural mode shape equations from the section V-b are substituted into the equation of motion. MATLAB codes given in Appendix-D are used to get the mass matrix, damping matrix, stiffness matrix, and force matrix required in the equation of motion. The forcing function in the equation of motion is $P=\int_{0}^{a} \int_{0}^{b} \sum_{l=1}^{N_{p}} P_{l}(t) \delta\left(x-\hat{x}_{l}(t)\right) \delta\left(y-\hat{y}_{l}(t)\right) w_{m n}(x, y) d x d y$, where $P_{l}(t)$ is the $l^{\text {th }}$ load moving on the bridge at time $t$. The movement of this $l^{\text {th }}$ load in the $\mathrm{X}$ and $\mathrm{Y}$ direction of the plate is represented by two Dirac-delta functions $\delta\left(x-\hat{x}_{l}(t)\right)$ and $\delta\left(y-\hat{y}_{l}(t)\right)$ respectively. Equation of motion given in Equation (5.13) is an ordinary differential equation and is solved by the Runge-Kutta method by using a MATLAB code shown in Appendix-D.

To check the accuracy of these two programs in MATLAB, a sample isotropic plate is considered and the results are compared with the responses in terms of vertical 
displacements investigated by Jong-Shyong Wu et. at. [15]. The dimensions and physical properties of the plate are $a=36 f t, b=12 f t, h=0.5 f t, v=0.3, E=4.32 \times 10^{9} p s f$, and $\rho=15.19$ slugs $/ \mathrm{ft}^{3}$. A single moving load with a magnitude of $20,000 \mathrm{lb}$ is considered to move with a speed of $36 \mathrm{ft} / \mathrm{sec}(24.69 \mathrm{mph})$ and $72 \mathrm{ft} / \mathrm{sec}(49.39 \mathrm{mph})$ on a center line of the plate. Figures 14 and 15 show response history of vertical displacements at the center of the plate with a load of magnitude $20,000 \mathrm{lb}$ moving at the speed of $36 \mathrm{ft} / \mathrm{sec}$ and $72 \mathrm{ft} / \mathrm{sec}$ respectively. Maximum vertical displacements from Figures 14 and 15 are compared with the displacements investigated by Jong-Shyong Wu et. at. [15] in Table 11.

Table 11: Comparison of maximum vertical displacements with the displacements investigated by Jong-Shyong Wu et. at. [15].

\begin{tabular}{cccc}
\hline $\begin{array}{c}\text { Speed } \\
\text { (ft/sec) }\end{array}$ & \multicolumn{3}{c}{ Vertical displacement (in.) } \\
\hline & MATLAB & Wu et. al. & \% difference \\
\hline 36 & 0.492 & 0.466 & 5.57 \\
72 & 0.624 & 0.610 & 2.29 \\
\hline
\end{tabular}

From Table 11 we can see that the results from the MATLAB program are in a good agreement with the displacements investigated by Jong-Shyong Wu et. at. [15]. Accuracy of the two programs for the orthotropic plates is checked by considering two different types of orthotropic plates. The time history of vertical displacements is compared with the results from the finite element analysis of the same plates using the finite element analysis package NISA [2]. 


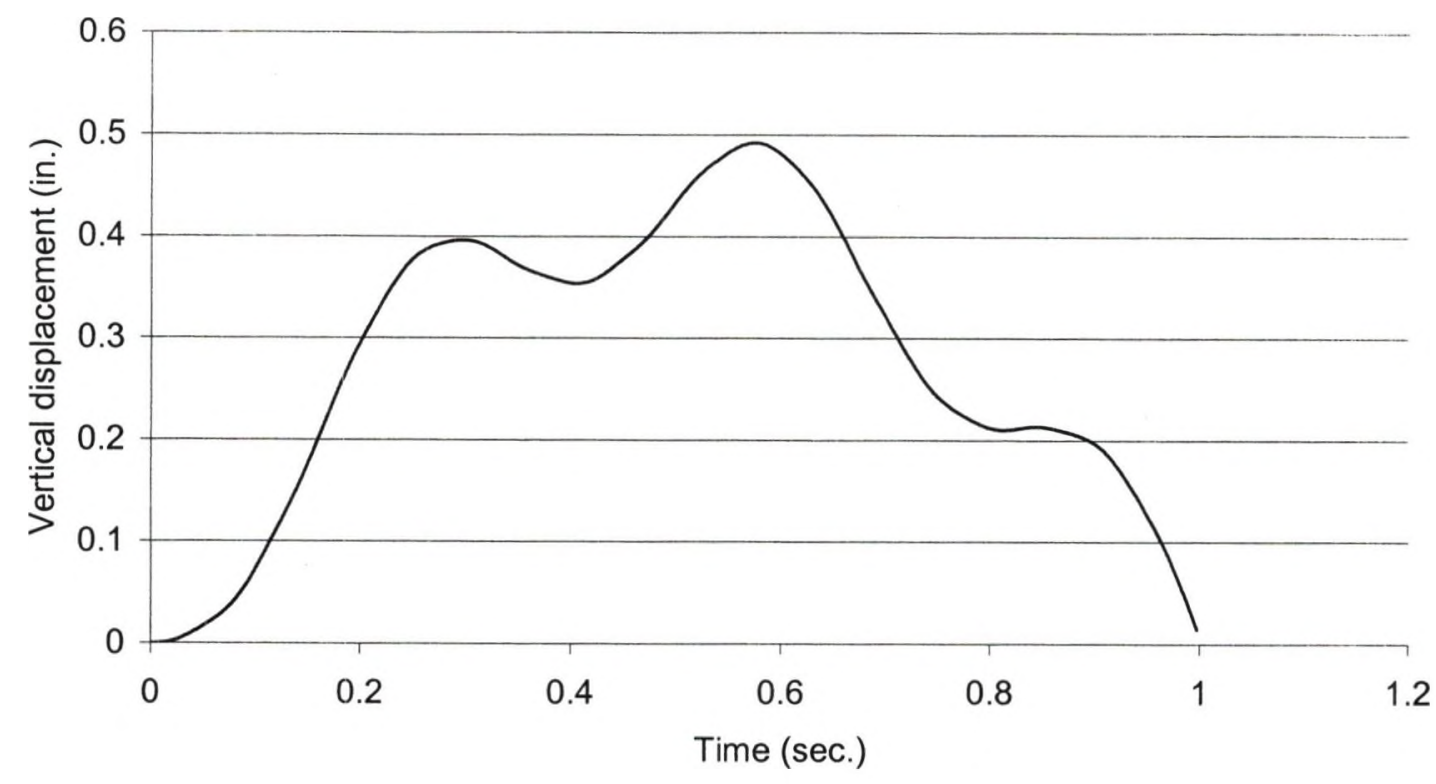

Figure 14: Vertical displacement history at the center of the plate with a load of magnitude 20,000 lb moving at the speed of $36 \mathrm{ft} / \mathrm{sec}(24.69 \mathrm{mph})$.

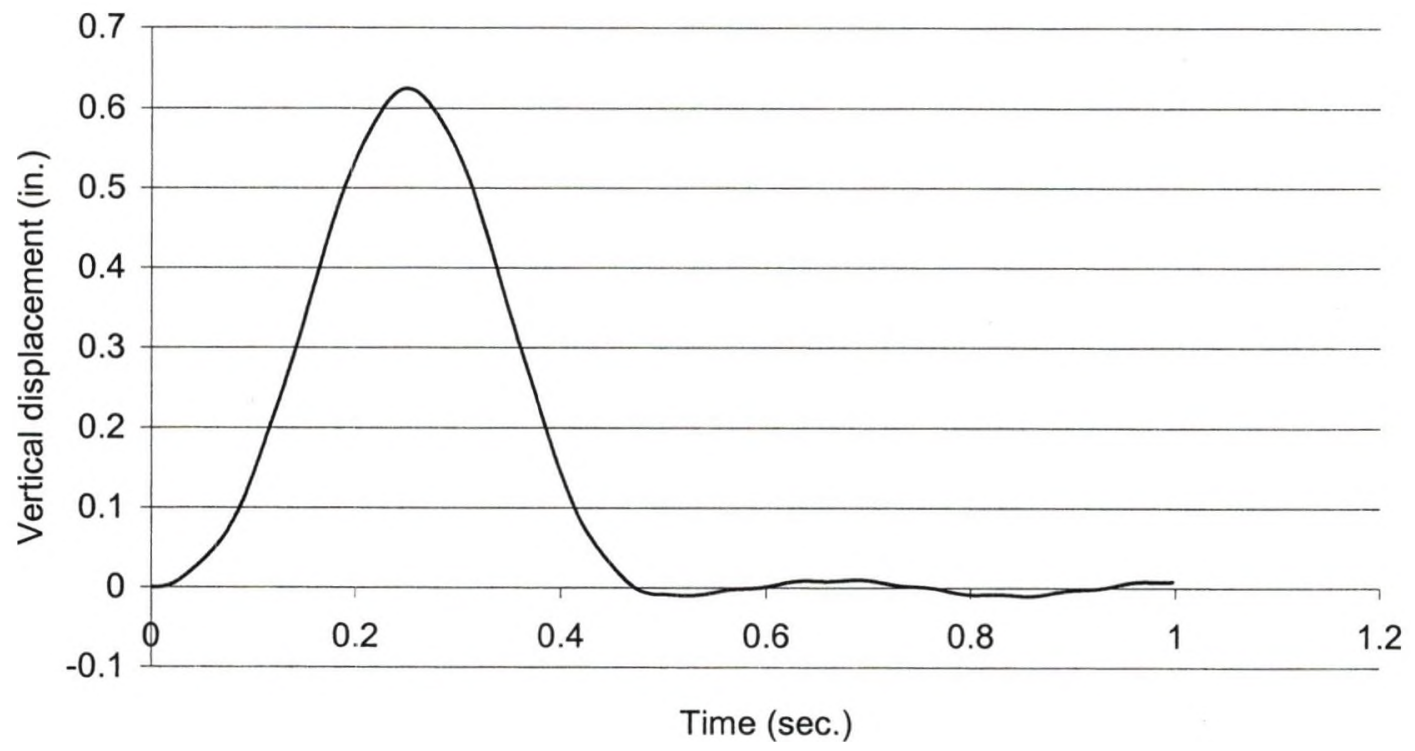

Figure 15: Vertical displacement history at the center of the plate with a load of magnitude 20,000 lb moving at the speed of $72 \mathrm{ft} / \mathrm{sec}(49.39 \mathrm{mph})$. 
The dimensions and physical properties of the first plate considered are $a=36 \mathrm{ft}$, $b=18 f t, h=0.5 f t, v_{x y}=0.3, D_{x}=5.06 \times 10^{7} l b . f t, D_{y}=10.12 \times 10^{7} l b . f t$, $H=20.24 \times 10^{7} l b . f t$, and $\rho=15.19$ slugs $/ f t^{3}$. A single moving load of magnitude 20,000 $\mathrm{lb}$ is considered moving with a speed of $36 \mathrm{ft} / \mathrm{sec}$. The Eigen-values are calculated by solving Equations (5.33), (5.38), (5.45), and (5.50). Table 12 gives the first four Eigenvalues of the plate.

Table 12: First four Eigen-values of the plate with $D_{x} / D_{y}=0.5 \& . H / D_{y}=2$.

\begin{tabular}{cc}
\hline Mode & $\lambda^{2}$ \\
\hline$(1,1)$ & 6.85 \\
$(1,2)$ & 41.75 \\
$(2,1)$ & 27.65 \\
$(2,2)$ & 85.45 \\
\hline
\end{tabular}

Figure 16 shows the comparison of the vertical displacement at the center of the plate with the displacement calculated by the finite element analysis in NISA [2].

The maximum vertical displacement from the MATLAB program is $0.328 \mathrm{in}$. where as it is 0.339 in. in NISA. As we can see the difference in the results is $3.8 \%$, that means the results from the program are acceptable. 


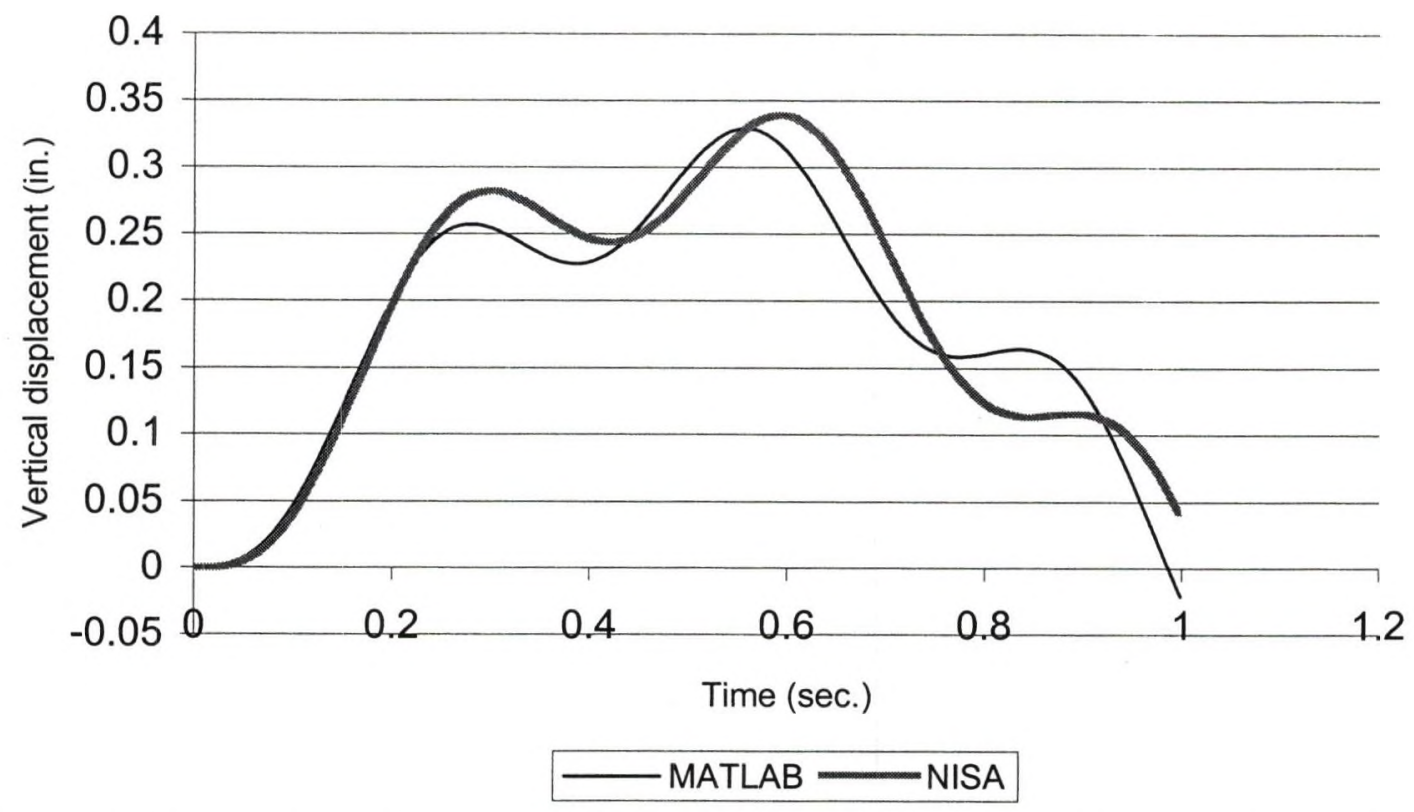

Figure 16: Comparison of the vertical displacement at the center of the first plate at the speed of $36 \mathrm{ft} / \mathrm{sec}(24.69 \mathrm{mph})$.

The dimensions and physical properties of the second plate considered are $a=36 f t, b=18 f t, h=0.5 f t, v_{x y}=0.3, D_{x}=10.12 \times 10^{7} l b . f t, D_{y}=5.06 \times 10^{7} l b . f t$, $H=1.5 \times 10^{7} l b . f t$, and $\rho=15.19$ slugs $/ f t^{3}$. A single moving load of magnitude 20,000 lb is considered moving with a speed of $36 \mathrm{ft} / \mathrm{sec}$. The Eigen-values are calculated by solving Equations (5.45), (5.50), (5.58), and (5.62). Table 13 gives the first four Eigenvalues of the plate.

Table 13: First four Eigen-values of the plate with $D_{x} / D_{y}=2.0 \& . H / D_{y}=0.3$.

\begin{tabular}{cc}
\hline Mode & $\lambda^{2}$ \\
\hline$(1,1)$ & 13.639 \\
$(1,2)$ & 249.25 \\
$(2,1)$ & 54.55 \\
$(2,2)$ & 261.15 \\
\hline
\end{tabular}


Figure 17 shows the comparison of the vertical displacement at the center of the plate with the displacement calculated by the finite element analysis in NISA [2]. The maximum vertical displacement from the MATLAB program is 0.086 in. where as it is 0.0825 in. in NISA. As we can see the difference in the results is $5.0 \%$, that means the results from the program are acceptable.

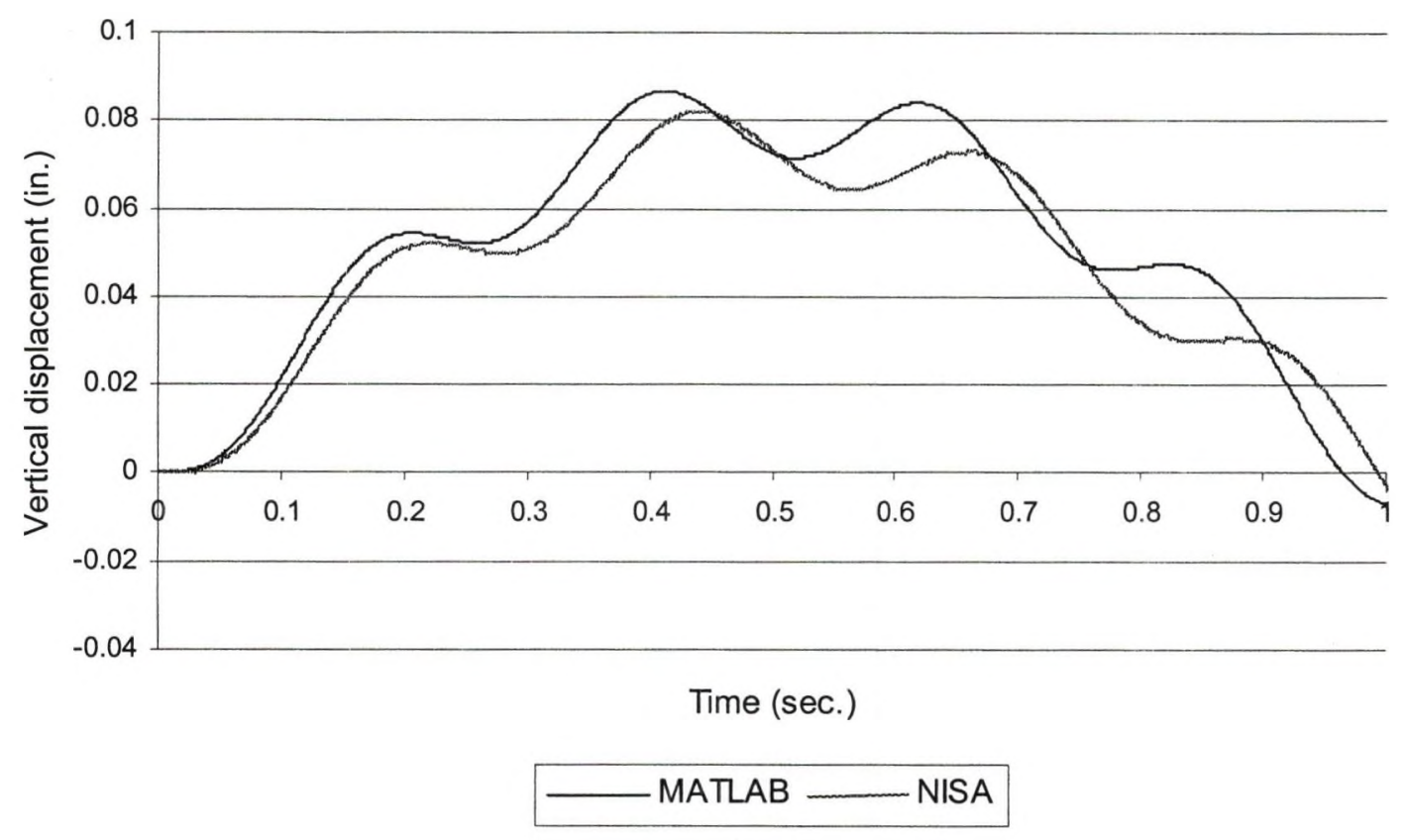

Figure 17: Comparison of the vertical displacement at the center of the second plate at the speed of $36 \mathrm{ft} / \mathrm{sec}(24.69 \mathrm{mph})$.

\section{Analysis of a T-beam Bridge under Moving Loads}

The equations derived earlier in the chapter for the deflection of an orthotropic plate are dependent upon the flexural rigidities $D x$ and $D y$ and the torsional rigidity $2 \mathrm{H}$ of the deck. These rigidities are the functions of the elastic properties of the material and the intersection of the individual beams and slab forming the orthotropic deck. Only 
the T-beam bridge decks are considered in this study. Figure 18 shows the general elements of a T-beam bridge deck. A generalized derivation of a T-beam bridge deck given by A.R. Cusens and R. P. Pama [16] is used to calculate the rigidities.

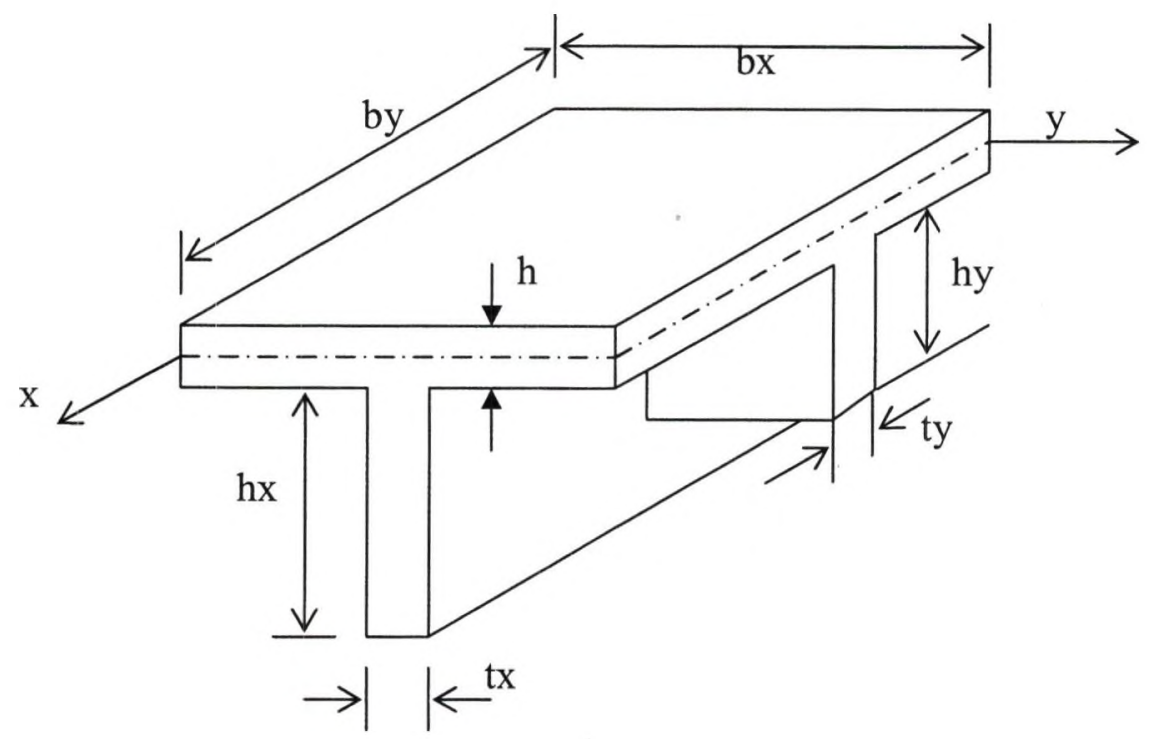

Figure 18: Element of T-beam bridge deck.

The flexural rigidities $D x$ and $D y$ are calculated from the second moment of area per unit length and the torsional rigidity $2 H$ is calculated as

$$
2 H \cong B_{x y}+B_{y x}+\frac{E t^{3}}{6}
$$

Where,

$B_{x y}=\frac{G k_{1} h_{x} t_{x}^{3}}{b_{x}}$ and $B_{y x}=\frac{G k_{1} h_{y} t_{y}^{3}}{b_{y}}$

A simply supported T-beam bridge deck as shown in Figure 19 is considered in this investigation. 


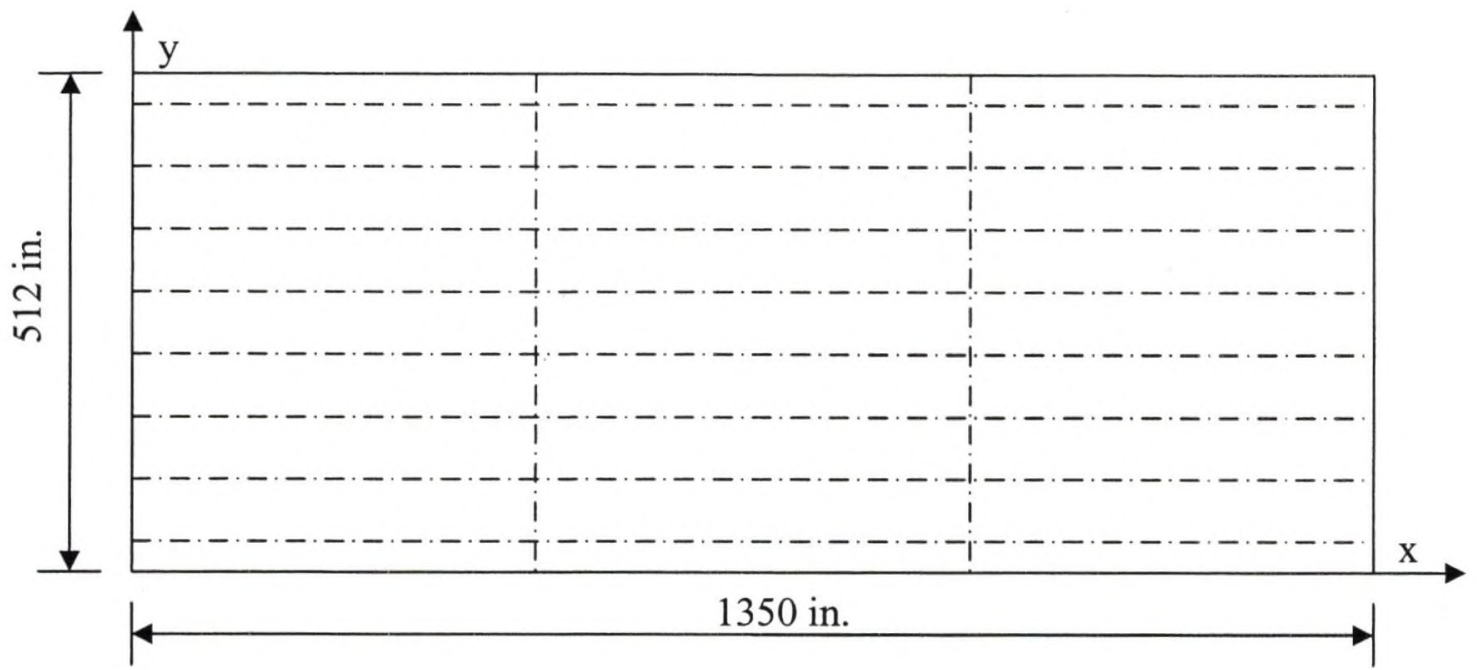

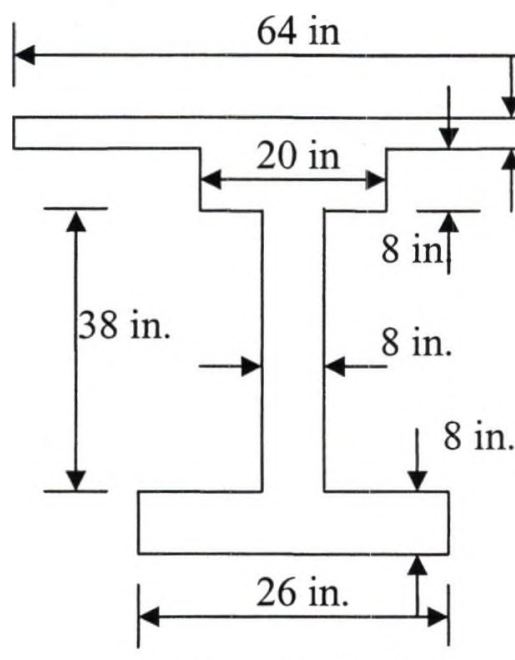

Longitudinal beam cross-section

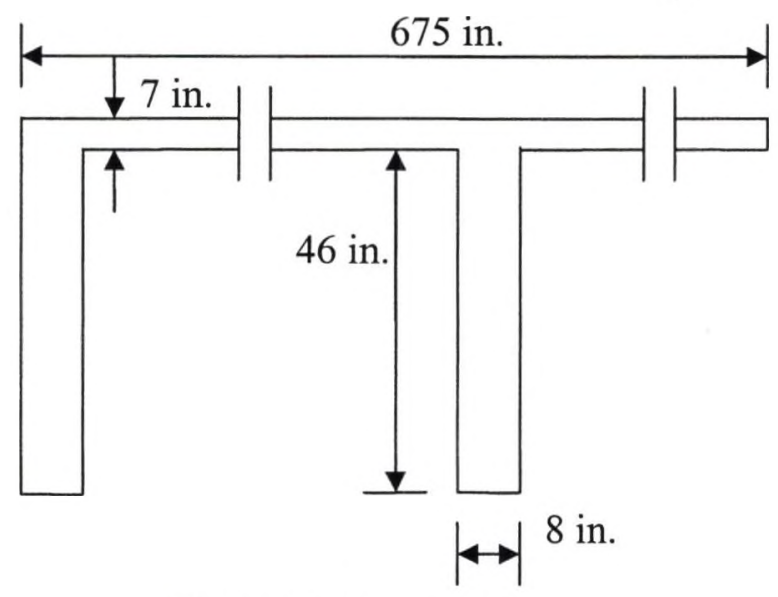

Transverse beam cross-section

Figure 19: Simply supported bridge considered in the investigation.

The flexural rigidities are calculated as,

$D_{x}=\frac{E I_{x}}{b_{x}}=7983.53 E$ and

$D_{y}=\frac{E I_{y}}{b_{y}}=883.36 E$

The torsional rigidity $H$ is calculated as

$2 H \cong B_{x y}+B_{y x}+\frac{E t^{3}}{6}$ 
$k_{1}$ in the terms $B_{x y}$ and $B_{y x}$ depends upon the ratio of $h$ and $t$ of the section, and is plotted by A.R. Cusens and R. P. Pama [16]. The value of $k_{1}$ is considered as 0.30 for the calculation of $B_{x y}$, and is considered as 0.29 for the calculation of $B_{y x}$.

The torsional rigidity becomes

$2 H \cong B_{x y}+B_{y x}+\frac{E t^{3}}{6}=117.72 E$

Modulus of elasticity for the bridge deck is considered as $E=3.5 \times 10^{6} \mathrm{psi}$ and the poisons ratio is considered as $v_{x y}=0.15$.

Which gives us

$D_{x}=2.3285 \times 10^{9} \mathrm{lb} . f t$

$D_{y}=0.2575 \times 10^{9} \mathrm{lb} . f t$

$2 H=0.0342 \times 10^{9} \mathrm{lb} . f t$

Eigen-values for this orthotropic plate are calculated by using the MATLAB program as For mode $(1,1)=29.64$.

mode $(1,2)=427.25$.

mode $(2,1)=118.55$.

mode $(2,2)=439.55$.

The rigidity ratios are $\frac{D_{x}}{D_{y}}=9.042, \frac{H}{D_{y}}=0.066$. According to these rigidity ratios, the mode shape categorization of the orthotropic plate for $m=1$ is If $0<\lambda^{2} \leq 29.670$, the orthotropic plate fall into category-II.

If $29.670<\lambda^{2} \leq 29.677$, the orthotropic plate fall into category-Ia. 
If $29.677<\lambda^{2}$, the orthotropic plate fall into category-Ib.

The mode shape categorization of the orthotropic plate for $m=2$ is

If $0<\lambda^{2} \leq 118.682$, the orthotropic plate fall into category-II.

If $118.682<\lambda^{2} \leq 118.711$, the orthotropic plate fall into category-Ia.

If $118.711<\lambda^{2}$, the orthotropic plate fall into category-Ib.

From the Eigen-values calculated from MATLAB program, we can see that the orthotropic plate in consideration falls into category-II for modes $(1,1)$ and $(2,1)$, and fall into category- $\mathrm{lb}$ for modes $(1,2)$ and $(2,2)$. A single load of magnitude $10,000 \mathrm{lb}$ is considered moving with different speeds on the center line of the bridge deck. Response in terms of vertical displacement is calculated by using the MATLAB program.

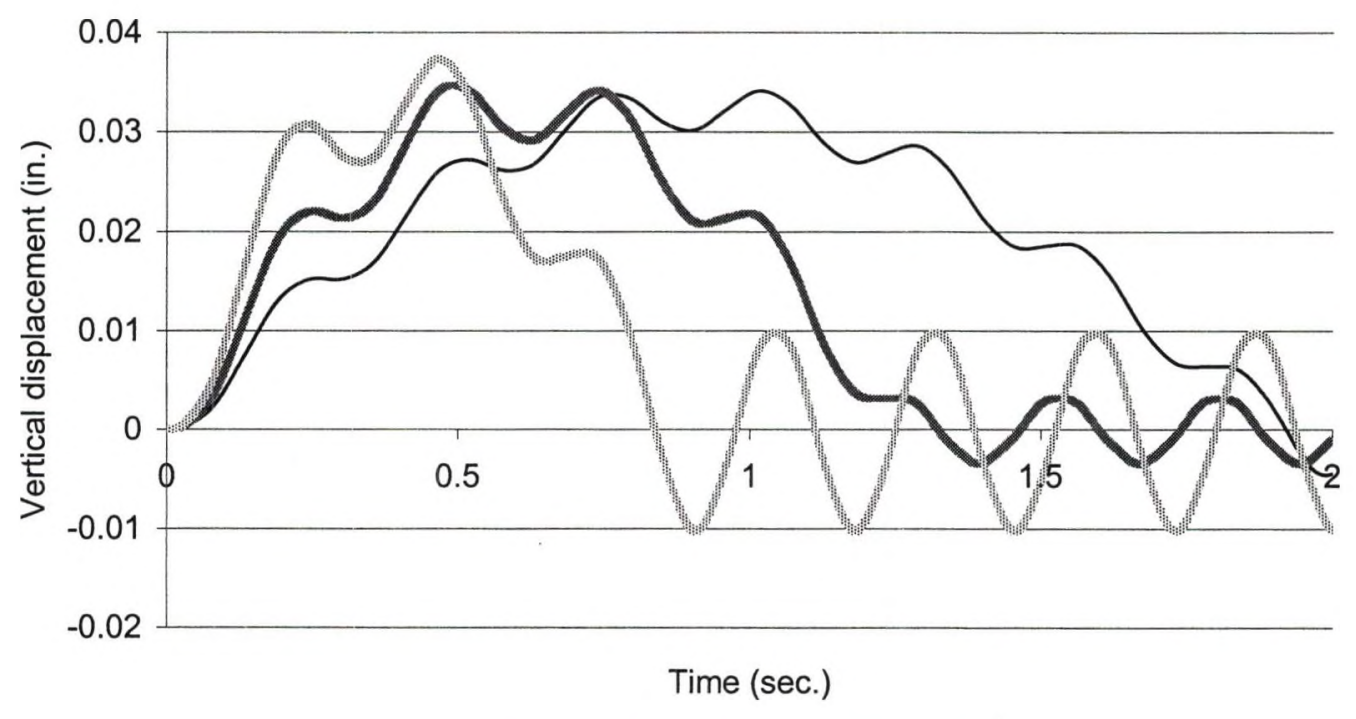

$-40 \mathrm{mph}=60 \mathrm{mph}$.

Figure 20: Time history of vertical displacement at the center of the T-beam bridge deck under a single moving load. 
Figure 20 shows the time history of vertical displacements at the center of the plate $(a / 2, b / 2)$ at the speed of 40,60 , and $90 \mathrm{mph}$. Table 14 summarizes the maximum vertical displacement at different speeds of a single moving load of magnitude 10,000 lb.

Table 14: Summary of maximum vertical displacement at the center of the T-beam bridge deck under a single moving load.

\begin{tabular}{cccc}
\hline Speed & $\begin{array}{c}\text { Vertical } \\
\text { displacement }\end{array}$ & $\begin{array}{c}\text { Static vertical } \\
\text { displacement }\end{array}$ & Impact \\
\hline $\mathrm{mph}$ & (in.) & (in.) & $\%$ \\
\hline 40 & 0.0340 & 0.0321 & 5.91 \\
45 & 0.0349 & 0.0321 & 8.72 \\
50 & 0.0354 & 0.0321 & 10.28 \\
55 & 0.0351 & 0.0321 & 9.34 \\
60 & 0.0346 & 0.0321 & 7.78 \\
65 & 0.0358 & 0.0321 & 11.52 \\
70 & 0.0367 & 0.0321 & 14.33 \\
75 & 0.0372 & 0.0321 & 15.88 \\
80 & 0.0375 & 0.0321 & 16.82 \\
85 & 0.0375 & 0.0321 & 16.82 \\
90 & 0.0373 & 0.0321 & 16.19 \\
\hline
\end{tabular}

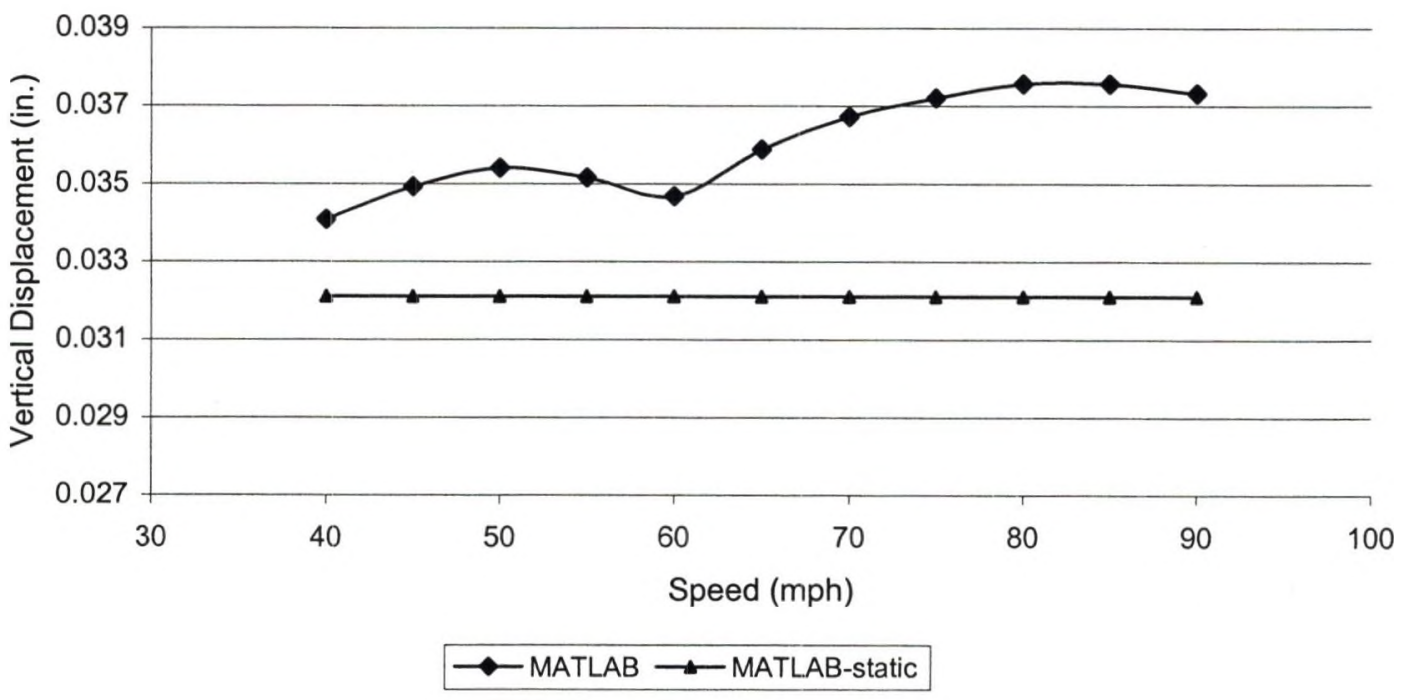

Figure 21: Comparison of maximum vertical displacement at the center of T-beam bridge deck at different speeds under a single moving load. 
Figure 21 shows the comparison of the maximum vertical displacement at the center of the T-beam bridge deck at different speeds. Static displacement in Table 14 is calculated by considering the speed of single moving load as $5 \mathrm{mph}$ in the MATLAB program. As we can see from the Table 14 and Figure 21, the maximum vertical displacement at the center of the T-beam bridge deck increases with the increase in the speed. The maximum impact is of $16.82 \%$ when the single moving of magnitude 10,000 $\mathrm{lb}$ is moving at a speed of $80 \mathrm{mph}$. To study the effect of multiple moving loads on the bridge deck, two loads of magnitude 10,000 lb each are considered moving at $10 \mathrm{ft}$ apart from each other on the center line of the T-beam bridge deck at different speeds. Figure 22 shows the times history of vertical displacement at the center of the bridge deck at the speed of 40,60 , and $90 \mathrm{mph}$.

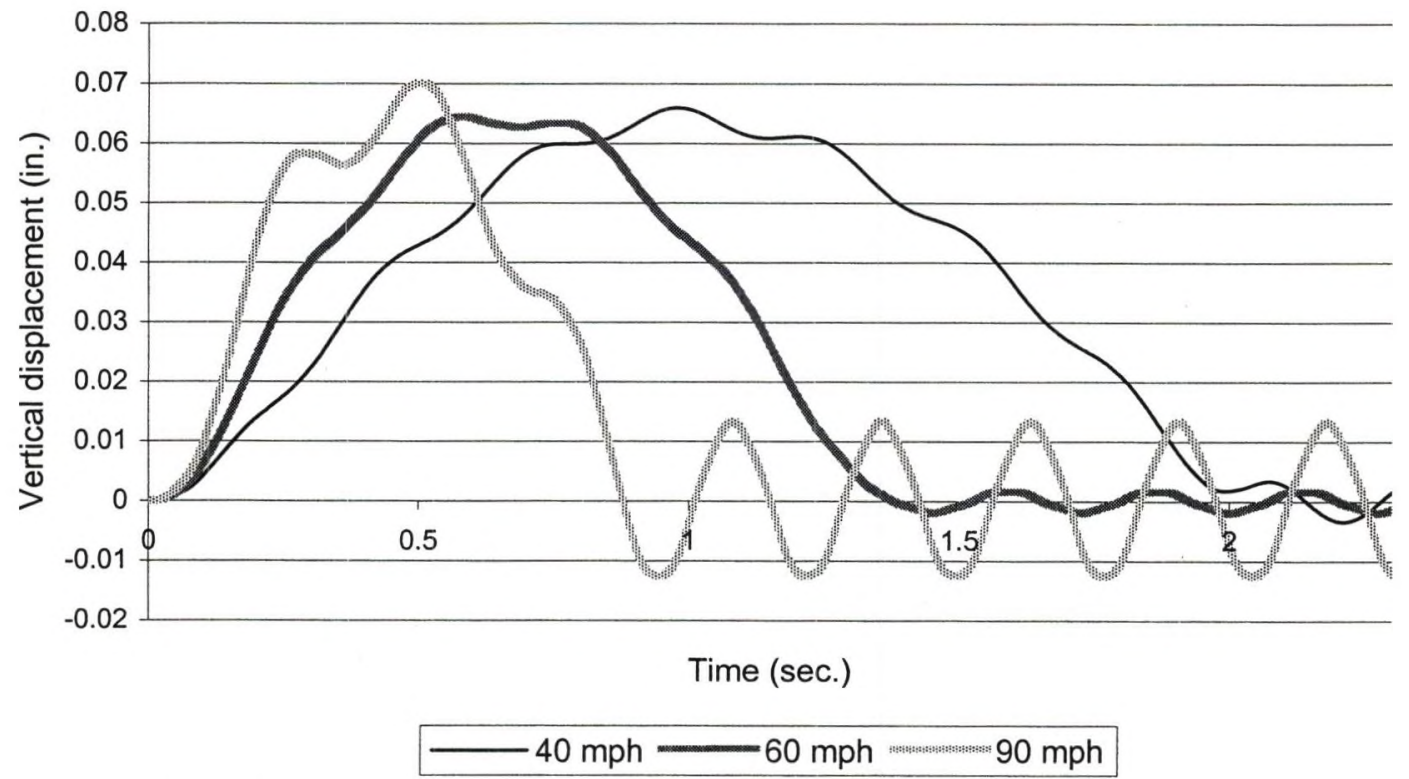

Figure 22: Time history of vertical displacement at the center of the T-beam bridge deck under two moving loads. 
Table 15 summarizes the maximum vertical displacement at the center of the bridge deck when two moving loads of magnitude $10,000 \mathrm{lb}$ each with $10 \mathrm{ft}$ apart are moving at different speeds.

Table 15: Summary of maximum vertical displacement at the center of the T-beam bridge deck under two moving loads.

\begin{tabular}{cccc}
\hline Speed & $\begin{array}{c}\text { Vertical } \\
\text { displacement }\end{array}$ & $\begin{array}{c}\text { Static vertical } \\
\text { displacement }\end{array}$ & Impact \\
\hline $\mathrm{mph}$ & (in.) & (in.) & $\%$ \\
\hline 40 & 0.0658 & 0.0642 & 2.49 \\
45 & 0.0646 & 0.0642 & 0.62 \\
50 & 0.0645 & 0.0642 & 0.46 \\
55 & 0.0645 & 0.0642 & 0.46 \\
60 & 0.0644 & 0.0642 & 0.31 \\
65 & 0.0664 & 0.0642 & 3.42 \\
70 & 0.0682 & 0.0642 & 6.23 \\
75 & 0.0694 & 0.0642 & 8.09 \\
80 & 0.0702 & 0.0642 & 9.34 \\
85 & 0.0704 & 0.0642 & 9.65 \\
90 & 0.0700 & 0.0642 & 9.03 \\
\hline
\end{tabular}

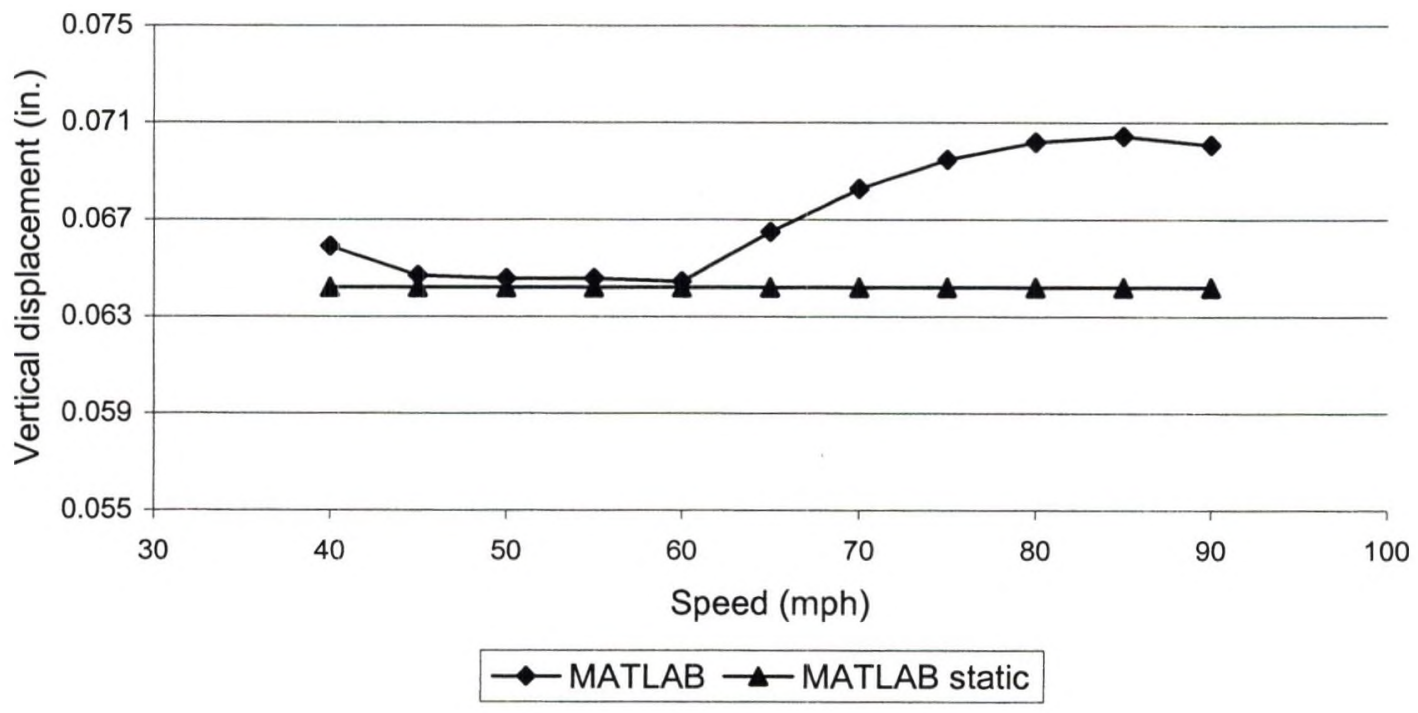

Figure 23: Comparison of maximum vertical displacement at the center of T-beam bridge deck at different speeds under two moving loads. 
Figure 23 shows the comparison of the maximum vertical displacement at the center of the T-beam bridge deck under two moving loads. Static displacement in Table 15 is calculated by considering the speed of two moving loads as $5 \mathrm{mph}$ following each other at $10 \mathrm{ft}$ apart in the MATLAB program. As we can see from the Table 15 and Figure 23, that the maximum impact is of $9.65 \%$ at the speed of $85 \mathrm{mph}$. From these two analyses of the T-beam bridge deck in consideration, we can see that the effect of the speed of the moving load on the vertical displacement at the center of the bridge deck is more for a single load of magnitude $10,000 \mathrm{lb}$ than for the two loads of magnitude $10,000 \mathrm{lb}$ each following each other at $10 \mathrm{ft}$ apart. 


\section{CHAPTER VI}

\section{FINITE ELEMENT ANALYSIS OF BRIDGES UNDER MOVING LAODS}

Finite element analysis is a strong method to solve the generalized problems in the field of engineering. The purpose of this part of the study is to investigate the behavior of bridges under moving loads by the use of finite element analysis and compare the results with the analytical method. NISA (Numerically Integrated System for Analysis) is one of the comprehensive suites of general purpose finite element analysis programs.

There are two ways to analysis a bridge by this method. First is to model the bridge by considering it as an orthotropic plate with material orthotrophy and the second is to model it in great details so that the geometrical orthotrophy is taken care of.

Figure 24 shows an orthotropic plate with a single moving load on it.

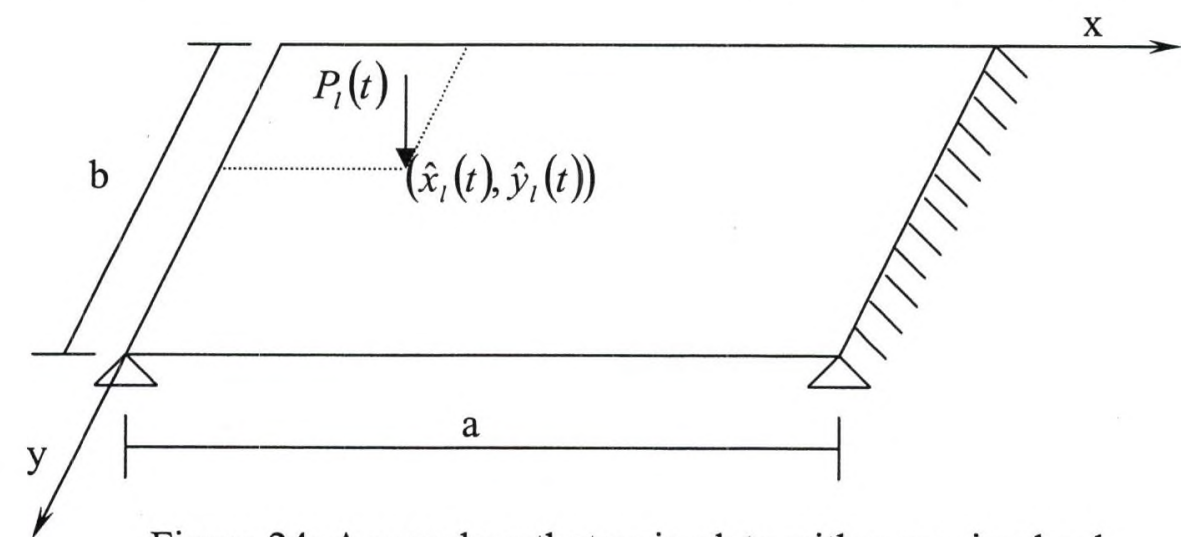

Figure 24: A sample orthotropic plate with a moving load 
A sample isotropic plate is considered for the analysis in NISA and the results are compared with the responses in terms of vertical displacements investigated by JongShyong Wu et. al. [15]. The dimensions and physical properties of the plate are $a=36 \mathrm{ft}$, $b=12 f t, h=0.5 f t, v=0.3, E=4.32 \times 10^{9} p s f$, and $\rho=15.19$ slugs $/ f^{3}$.

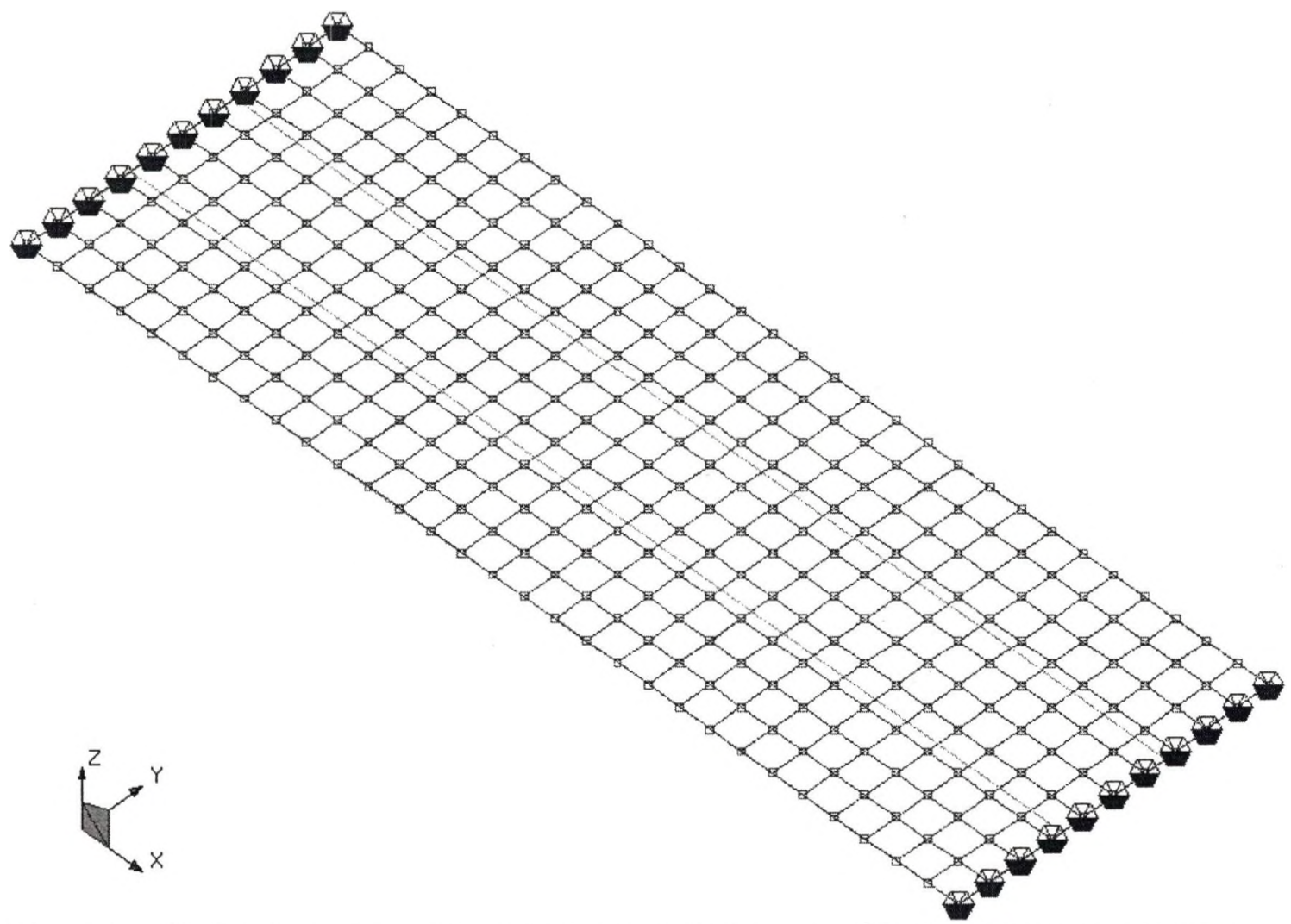

Figure 25: Finite element model of isotropic plate by using 3D shell elements in NISA.

Figure 25 shows the finite element model of the isotropic plate in consideration in NISA. The 3D shell elements are used to model the plate in NISA with a uniform thickness of $0.5 f t$ at all the edges. Modulus of elasticity, poison's ratio, and mass density of the material is provided. First of all, Eigen-value analysis is done to calculate the natural frequencies and modal shapes of the plate. A single moving load with a magnitude of $20,000 \mathrm{lb}$ is considered to move with a speed of $36 \mathrm{ft} / \mathrm{sec}$ and $72 \mathrm{ft} / \mathrm{sec}$ on a centerline of the plate. Moving load is simulated in NISA by using arrival time data 
and time function data for different speeds and the plate is then solved by modal superposition method to calculate the responses.

First four mode shapes of the isotropic plate in consideration are shown in Figures B.1 to B.4 in Appendix-B and the first four natural frequencies calculated by the Eigenvalue analysis are shown in Table 16.

Table 16: Comparison of the first four natural frequencies of the isotropic plate from Eigen-value analysis in NISA with the Eigen-values calculated in MATLAB.

\begin{tabular}{cccc}
\hline \multirow{2}{*}{ Modes } & $\begin{array}{c}\text { Natural frequency } \\
\text { from NISA }(\mathrm{Hz})\end{array}$ & \multicolumn{2}{c}{ Eigen-values } \\
\cline { 3 - 4 } & 2.9683 & NISA & MATLAB \\
\hline$(1,1)$ & 12.3302 & 9.472 & 9.465 \\
$(1,2)$ & 12.0205 & 39.347 & 39.865 \\
$(2,1)$ & 26.8394 & 38.360 & 38.235 \\
$(2,2)$ & & 85.650 & 86.545 \\
\hline
\end{tabular}

We get the natural frequency $(f)$ of the plate in cycles/sec $(\mathrm{Hz})$ in NISA, which is converted in to radians/sec $(\omega=2 \pi f)$. Eigen-values of the plate are calculated as $\lambda^{2}=\omega a^{2} \sqrt{\frac{\rho h}{D}}$. As we can see from the Table 16, Eigen-values calculated from NISA are in well agreement with that are calculated by using the programs developed in MATLAB.

Figures 26 and 27 show the response history of vertical displacements at the center of the plate (node \# 171) with a load of magnitude 20,000 lb moving at the speed of $36 \mathrm{ft} / \mathrm{sec}$ and $72 \mathrm{ft} / \mathrm{sec}$ respectively. 


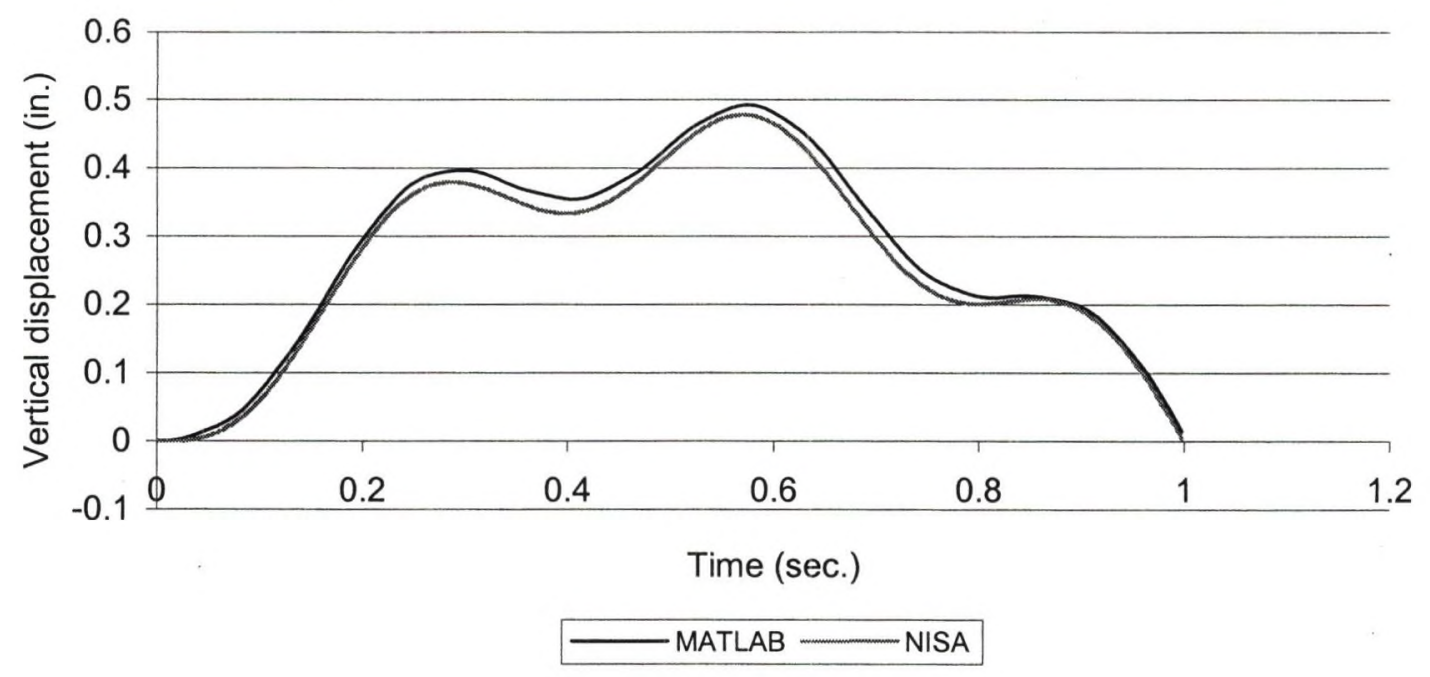

Figure 26: Time history of the vertical displacements at the center of the plate with a load of magnitude $20,000 \mathrm{lb}$ moving at the speed of $36 \mathrm{ft} / \mathrm{sec}$ in NISA.

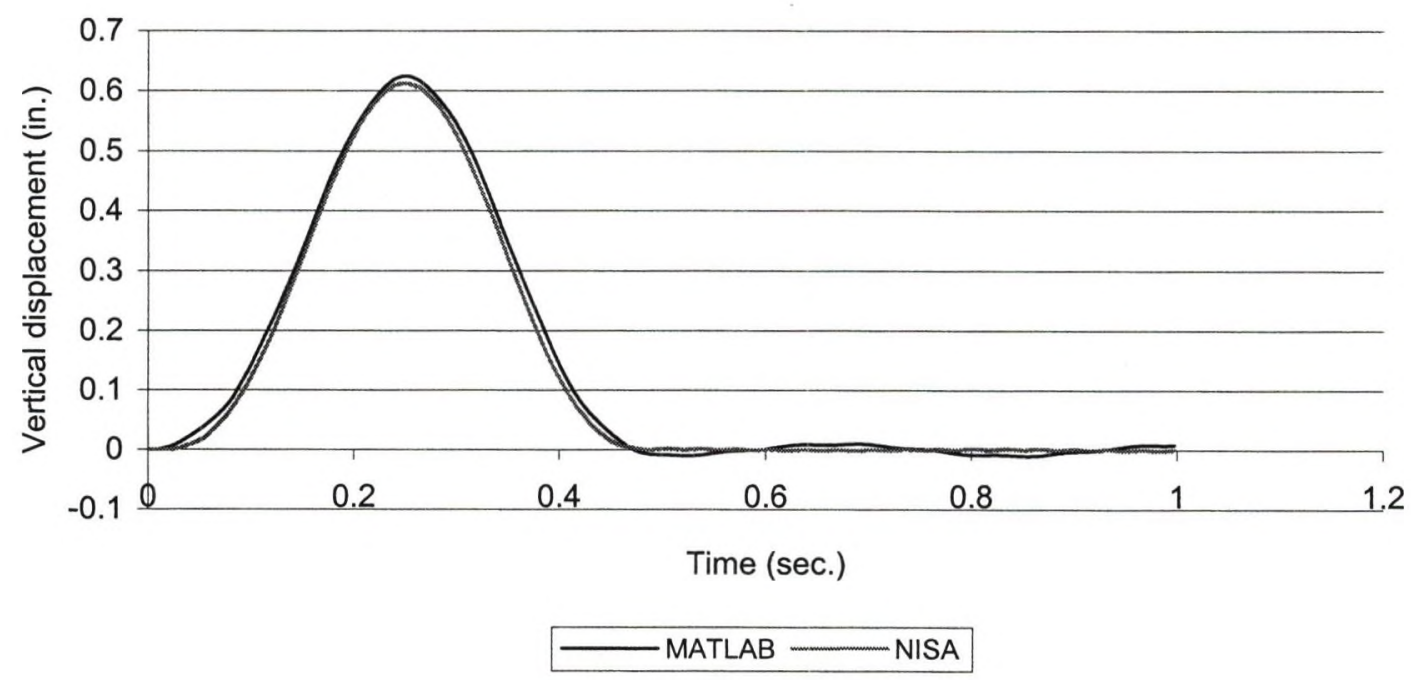

Figure 27: Time history of the vertical displacements at the center of the plate with a load of magnitude 20,000 lb moving at the speed of $72 \mathrm{ft} / \mathrm{sec}$ in NISA.

Maximum vertical displacements from Figures 26 and 27 are compared with the displacements calculated by using MATLAB program, and with the displacements investigated by Jong-Shyong Wu et. al. [15] in Table 17. 
Table 17: Comparison of maximum vertical displacements with the displacements investigated by Jong-Shyong Wu et. al. [15].

\begin{tabular}{cccc}
\hline $\begin{array}{c}\text { Speed } \\
\text { (ft/sec) }\end{array}$ & \multicolumn{3}{c}{ Vertical displacement (in.) } \\
\hline & MATLAB & Wu et. al. & NISA \\
\hline 36 & 0.492 & 0.466 & 0.477 \\
72 & 0.624 & 0.610 & 0.612 \\
\hline
\end{tabular}

From Table 17 we can see that the results from the finite element analysis program NISA are in a good agreement with the displacements investigated by Jong-Shyong Wu et. al. [15]. The T-beam bridge deck as shown in Figure 19 is modeled as an orthotropic plate. The flexural rigidities and torsional rigidity of the bridge deck are $D_{x}=2.3285 \times 10^{9} \mathrm{lb} . f t, D_{y}=0.2575 \times 10^{9} \mathrm{lb} . f t$, and $2 \mathrm{H}=0.0342 \times 10^{6} \mathrm{lb} . f t$. Modulus of elasticities of the plate are calculated as

$$
\begin{aligned}
& E_{x}=\frac{12 D_{x}\left(1-v_{x y} v_{y x}\right)}{h^{3}}=1.404 \times 10^{11} l b / f t^{2} \text {, and } \\
& E_{y}=\frac{D_{y} 12\left(1-v_{x y} v_{y x}\right)}{h^{3}}=0.1552 \times 10^{11} l b / f t^{2}
\end{aligned}
$$

The dimensions and physical properties of the plate are $a=112.5 \mathrm{ft}, b=42.6 \mathrm{ft}$, $h=0.58 f t, v=0.2, \rho=4.658$ slugs $/ f t^{3}$.

A single load of magnitude $10,000 \mathrm{lb}$ is considered moving with different speeds on the centerline of the bridge deck. Response in terms of vertical displacement is calculated by using the finite element analysis program NISA. 


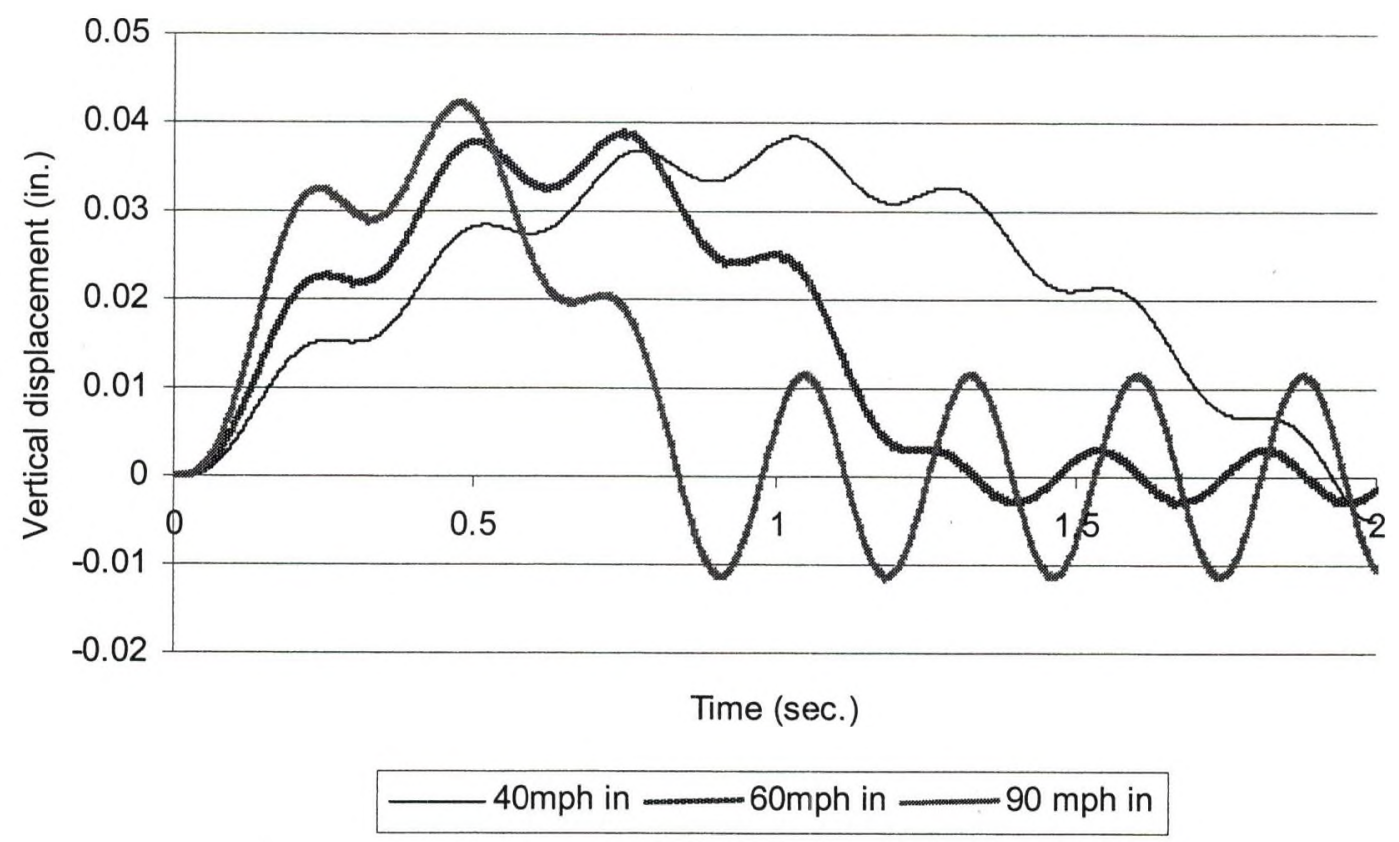

Figure 28: Time history of vertical displacements at the center of the T-beam bridge deck under a single moving load in NISA.

Figure 28 shows the time history of vertical displacements at the center of the plate (node \# 536) at the speed of 40,60 , and $90 \mathrm{mph}$. Table 18 summarizes the maximum vertical displacement at different speeds of a single moving load of magnitude 10,000 lb.

Table 18: Summary of maximum vertical displacement at the center of the T-beam bridge deck under a single moving load in NISA.

\begin{tabular}{cccc}
\hline Speed & $\begin{array}{c}\text { Vertical } \\
\text { displacement }\end{array}$ & $\begin{array}{c}\text { Static vertical } \\
\text { displacement }\end{array}$ & Impact \\
\hline $\mathrm{mph}$ & (in.) & (in.) & $\%$ \\
\hline 40 & 0.0384 & 0.0362 & 6.070 \\
45 & 0.0385 & 0.0362 & 6.353 \\
50 & 0.0394 & 0.0362 & 8.839 \\
55 & 0.0394 & 0.0362 & 8.839 \\
60 & 0.0386 & 0.0362 & 6.629 \\
65 & 0.0392 & 0.0362 & 8.287 \\
70 & 0.0404 & 0.0362 & 11.602 \\
75 & 0.0412 & 0.0362 & 13.812 \\
80 & 0.0418 & 0.0362 & 15.469 \\
85 & 0.0421 & 0.0362 & 16.298 \\
90 & 0.0420 & 0.0362 & 16.022 \\
\hline
\end{tabular}




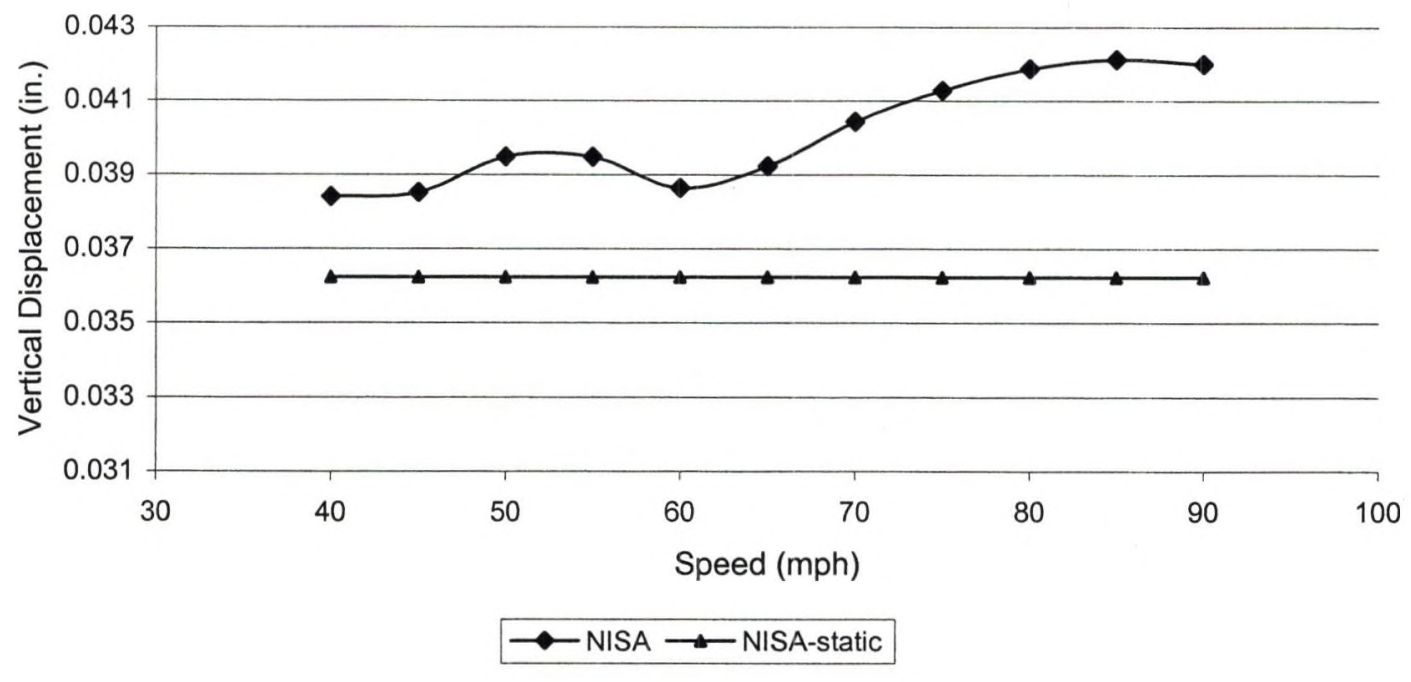

Figure 29: Comparison of maximum vertical displacement at the center of T-beam bridge deck at different speeds under a single moving load in NISA.

Figure 29 shows the comparison of the maximum vertical displacement at the center of the T-beam bridge deck at different speeds. Static displacement in Table 18 is calculated by considering the speed of single moving load as $5 \mathrm{mph}$. As we can see from the Table 18 and Figure 29, the maximum vertical displacement at the center of the Tbeam bridge deck increases with the increase in the speed. The maximum impact is of $16.29 \%$ when the single moving of magnitude $10,000 \mathrm{lb}$ is moving at a speed of $85 \mathrm{mph}$. To study the effect of multiple moving loads on the bridge deck, two loads of magnitude $10,000 \mathrm{lb}$ each are considered moving at $10 \mathrm{ft}$ apart from each other on the centerline of the T-beam bridge deck at different speeds. Figure 30 shows the times history of vertical displacement at the center of the bridge deck (node \# 536) at the speed of 40, 60, and 90 mph. 


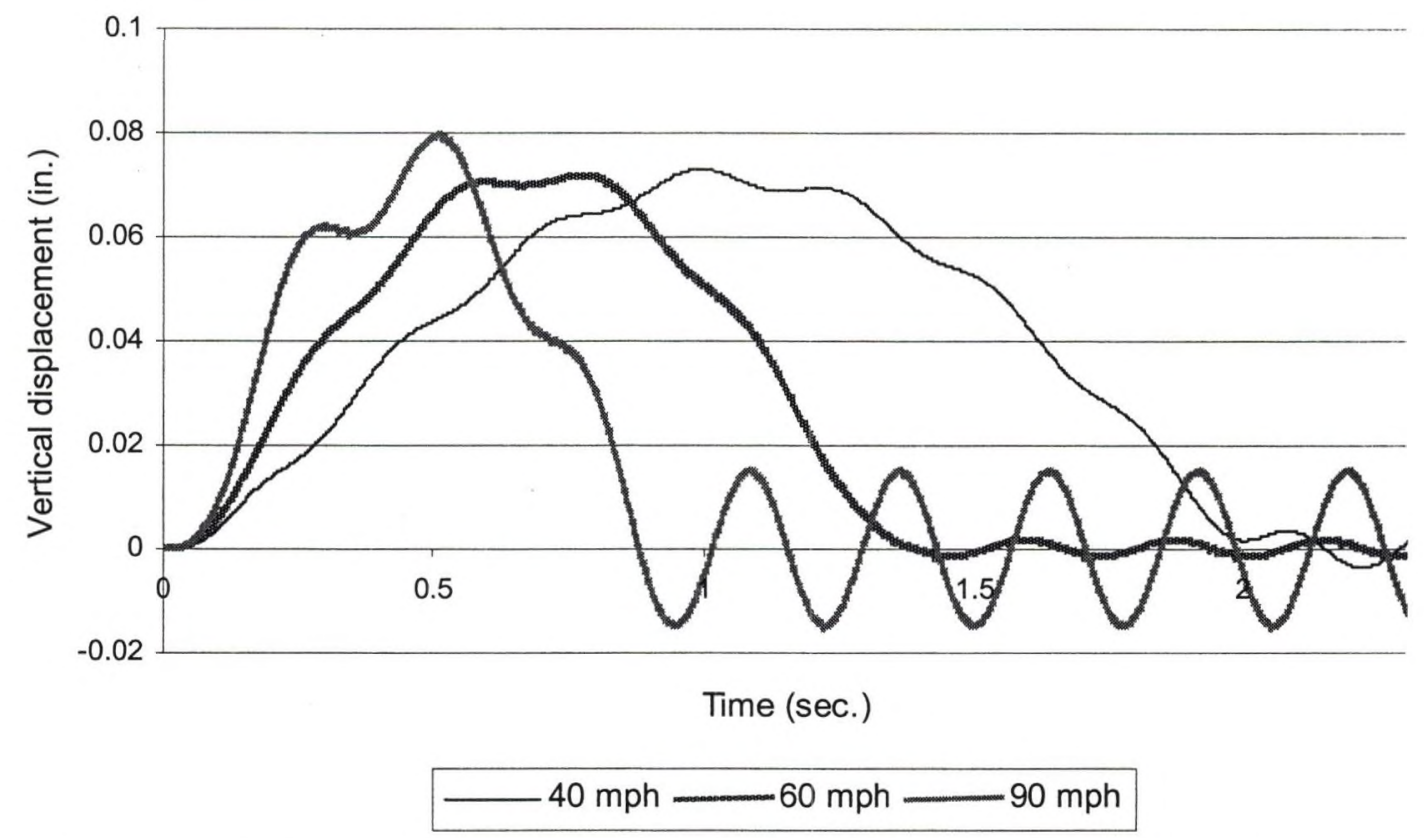

Figure 30: Time history of vertical displacements at the center of the T-beam bridge deck under two moving loads in NISA.

Table 19 summarizes the maximum vertical displacement at the center of the bridge deck when two moving loads of magnitude $10,000 \mathrm{lb}$ each with $10 \mathrm{ft}$ apart are moving at different speeds.

Table 19: Summary of maximum vertical displacement at the center of the T-beam bridge deck under two moving loads in NISA.

\begin{tabular}{cccc}
\hline Speed & $\begin{array}{c}\text { Vertical } \\
\text { displacement }\end{array}$ & $\begin{array}{c}\text { Static vertical } \\
\text { displacement }\end{array}$ & Impact \\
\hline $\mathrm{mph}$ & (in.) & (in.) & $\%$ \\
\hline 40 & 0.0729 & 0.0716 & 1.815 \\
45 & 0.0722 & 0.0716 & 0.837 \\
50 & 0.0718 & 0.0716 & 0.279 \\
55 & 0.0724 & 0.0716 & 1.117 \\
60 & 0.0716 & 0.0716 & 0.000 \\
65 & 0.0729 & 0.0716 & 1.815 \\
70 & 0.0752 & 0.0716 & 5.027 \\
75 & 0.0771 & 0.0716 & 7.681 \\
80 & 0.0784 & 0.0716 & 9.497 \\
85 & 0.0792 & 0.0716 & 10.614 \\
90 & 0.0793 & 0.0716 & 10.754 \\
\hline
\end{tabular}




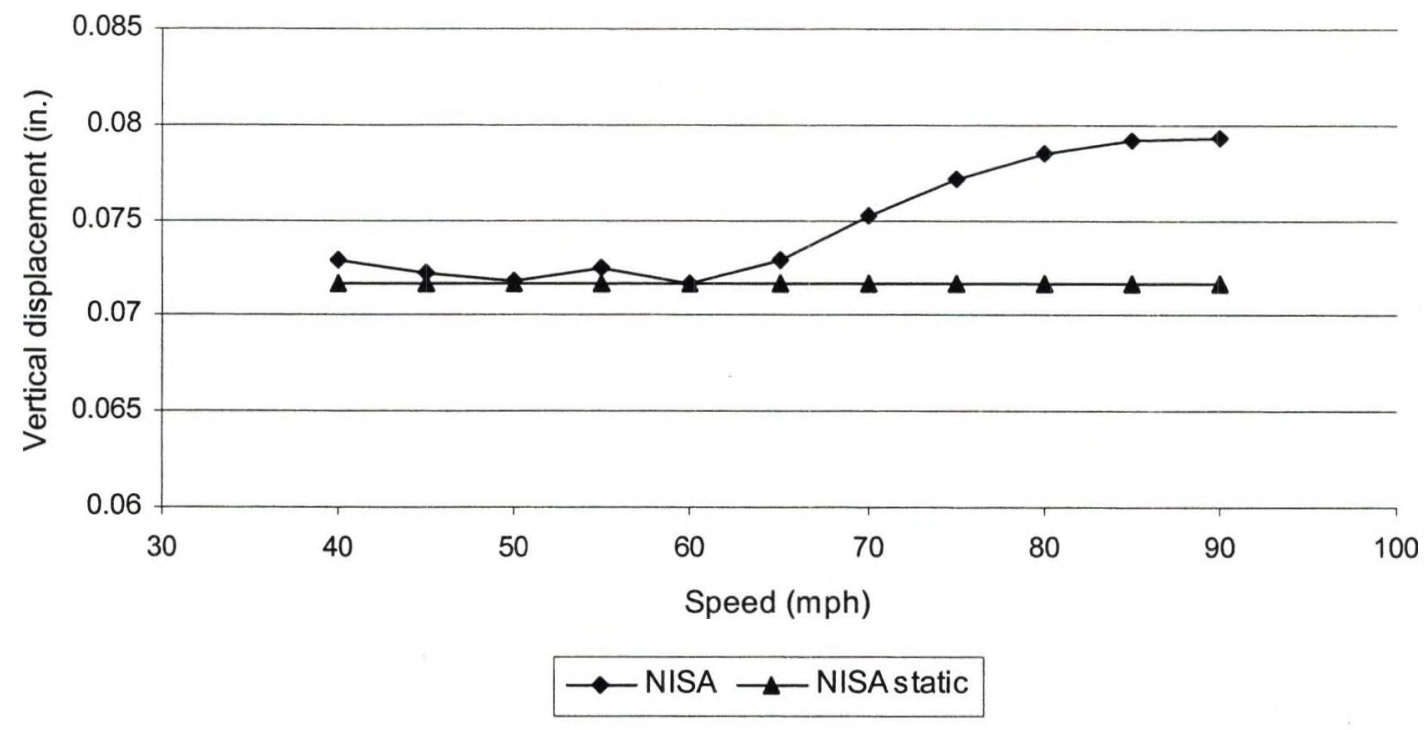

Figure 31: Comparison of maximum vertical displacement at the center of T-beam bridge deck at different speeds under two moving loads in NISA.

Figure 31 shows the comparison of the maximum vertical displacement at the center of the T-beam bridge deck under two moving loads. Static displacement in Table 19 is calculated by considering the speed of two moving loads as $5 \mathrm{mph}$ following each other at $10 \mathrm{ft}$ apart. As we can see from the Table 19 and Figure 31, that the maximum impact is of $10.754 \%$ at the speed of $90 \mathrm{mph}$. From these two analyses of the T-beam bridge deck in consideration, we can see that the effect of the speed of the moving load on the vertical displacement at the center of the bridge deck is more for a single load of magnitude $10,000 \mathrm{lb}$ than for the two loads of magnitude $10,000 \mathrm{lb}$ each following each other at $10 \mathrm{ft}$ apart. 


\section{CHAPTER VII}

\section{ANALYSIS OF A T-BEAM BRIDGE UNDER A MOVING AASSHTO HS20-44 TRUCK LOAD}

The objective of this research work is to study the cumulative effect of the different factors such as road surface roughness, vehicle dynamics, vehicle weight, and vehicle speed on the displacement of the bridge and compare the results with the impact factor given by AASHTO. In the previous chapters we have seen the effect of all these factors individually. To see the cumulative effect of all these factors on the bridge dynamics, a simply supported T-beam bridge deck as shown in Figure 32 is considered in this investigation. Span length of the bridge in consideration is $112.5 \mathrm{ft}$ (1350 in.) and width is $42.66 \mathrm{ft}$ (512 in.). The T-beam bridge under the investigation is assumed to be built on a motorway with a very good road condition. Only a single AASHTO HS20-44 truck load is considered moving along the centerline of the bridge deck at different speeds. 

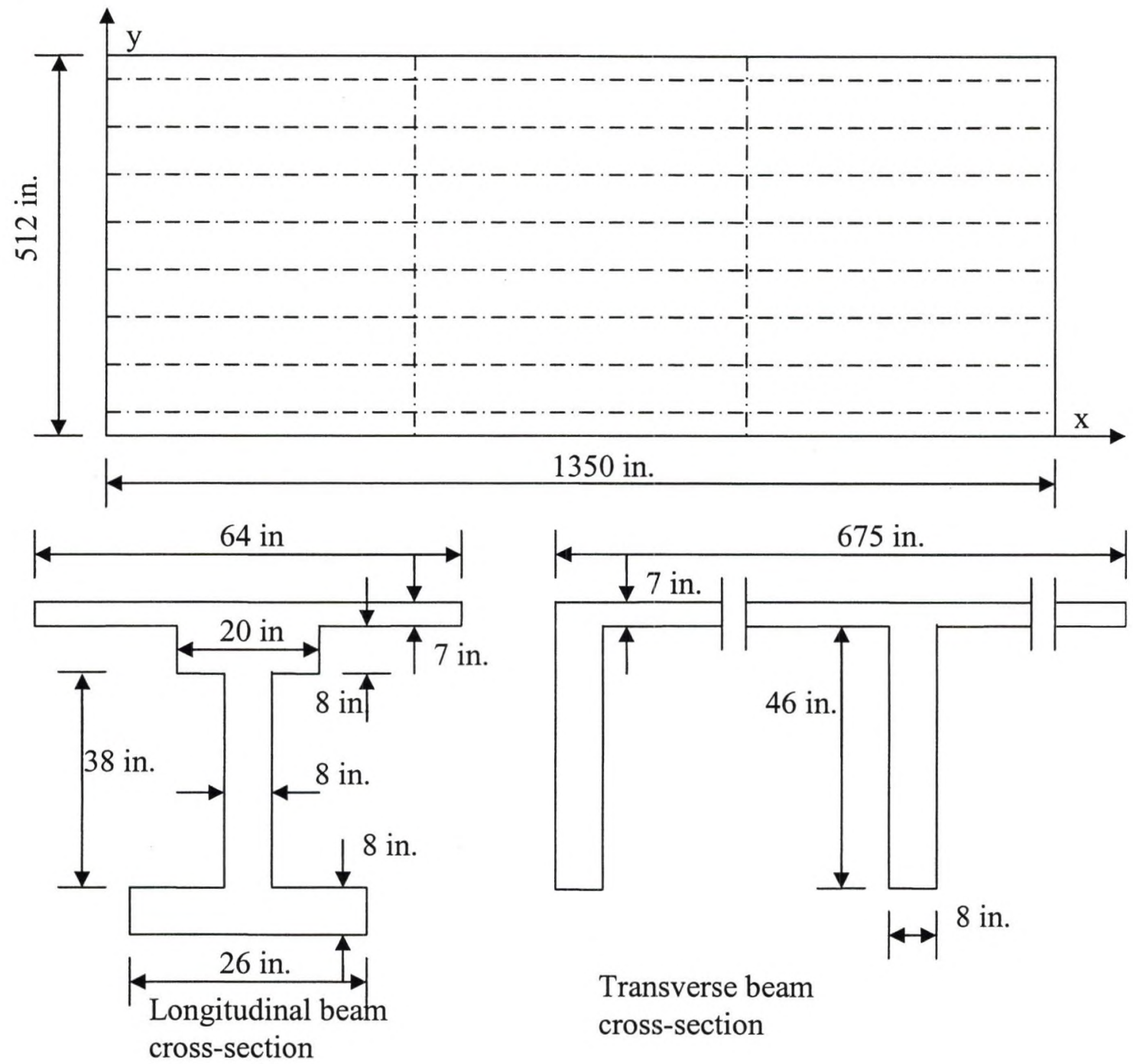

Transverse beam cross-section

Figure 32: Simply supported bridge considered in the investigation. 
VII-a: Generation of Road Surface Roughness for the T-beam Bridge Deck.

Road surface roughness is generated for this bridge deck by using the Power Spectral Density function as shown in Chapter III. The roughness coefficient $A$ according to the Table 1 is $5 \times 10^{-6} \mathrm{~m}^{3} /$ cycle. Span length of the bridge is $a=112.5 f t=34.298 \mathrm{~m}$.

Variance $\sigma^{2}$ required to generate a series of random numbers is calculated by using Equation 3.10.

$$
\sigma^{2}=\frac{A \lambda}{2}
$$

Where,

$$
\begin{aligned}
& A=5 \times 10^{-6} \\
& \lambda=34.298 / 2048=0.0167
\end{aligned}
$$

This gives us $\sigma^{2}=\frac{5 \times 10^{-6} \times 0.0167}{2}=0.041 \times 10^{-6}$

A series of random numbers which have approximate white noise properties with zero mean and variance $\sigma^{2}=0.041 \times 10^{-6}$ are generated in MATLAB and passed through a first order digital filter. The output function of this filter gives us the road surface roughness. Figure 33 shows the spectrum of the random numbers generated in MATLAB. Figure 34 shows the comparison of the PSD of the input spectrum with the approximate PSD of the white noise spectrum. 


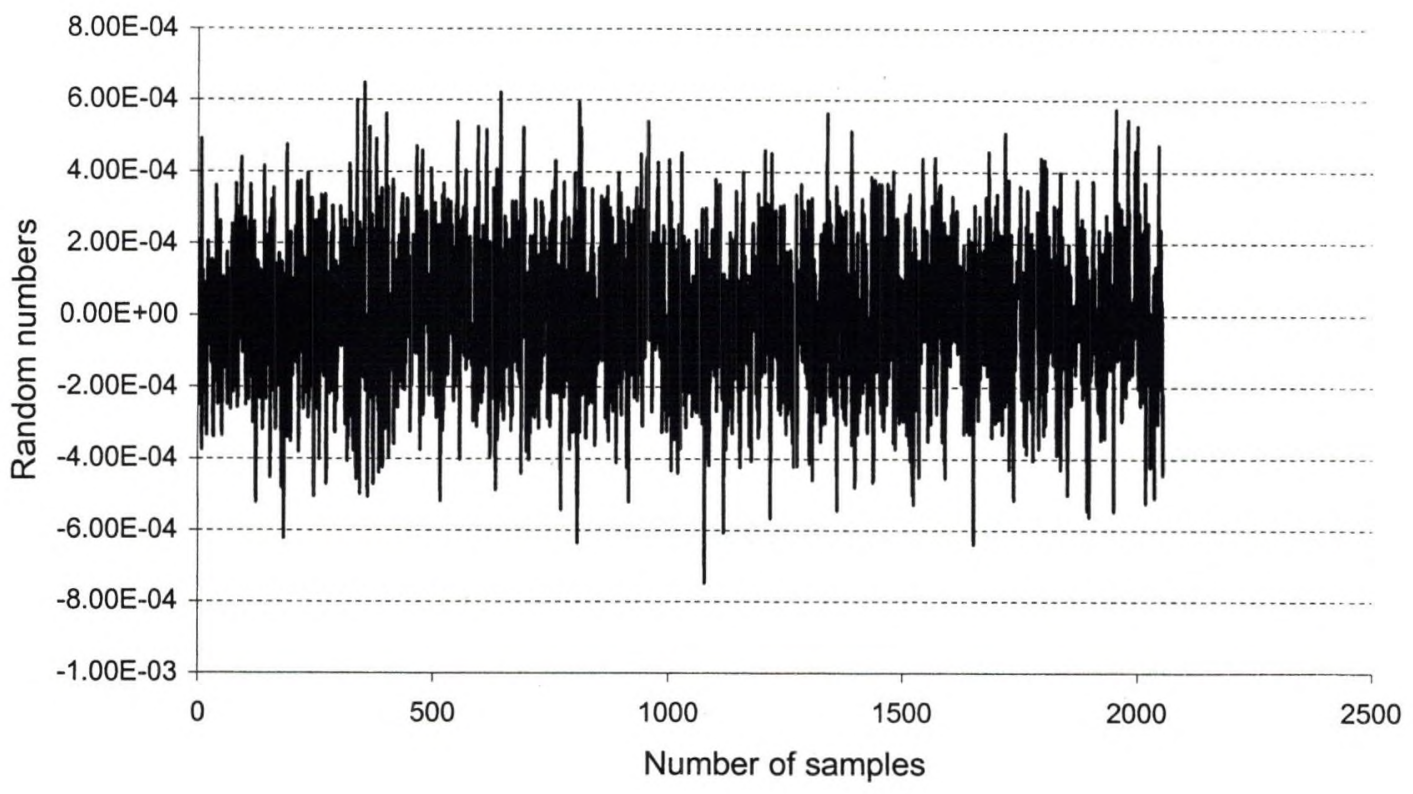

Figure 33: Spectrum of random numbers.

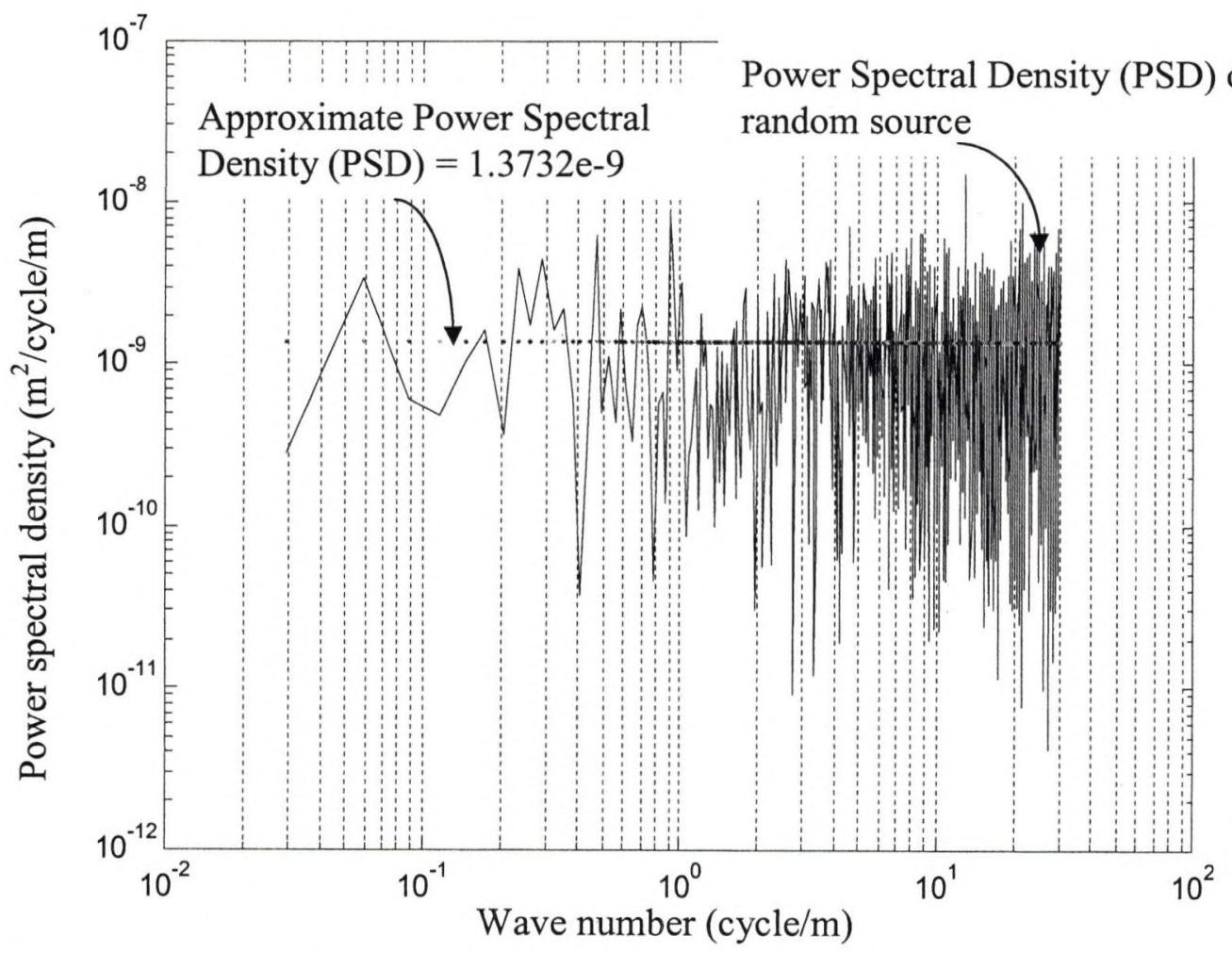

Figure 34: Comparison of Power Spectral Density of a random spectrum with the approximate Power Spectral Density. 
From Figure 34 we can see that the approximate power spectral density of the white noise spectrum is in agreement with the power spectral density of the input spectrum generated in MATLAB.

The output function of the first order digital filter gives us the road surface roughness and is shown in Figure 35.

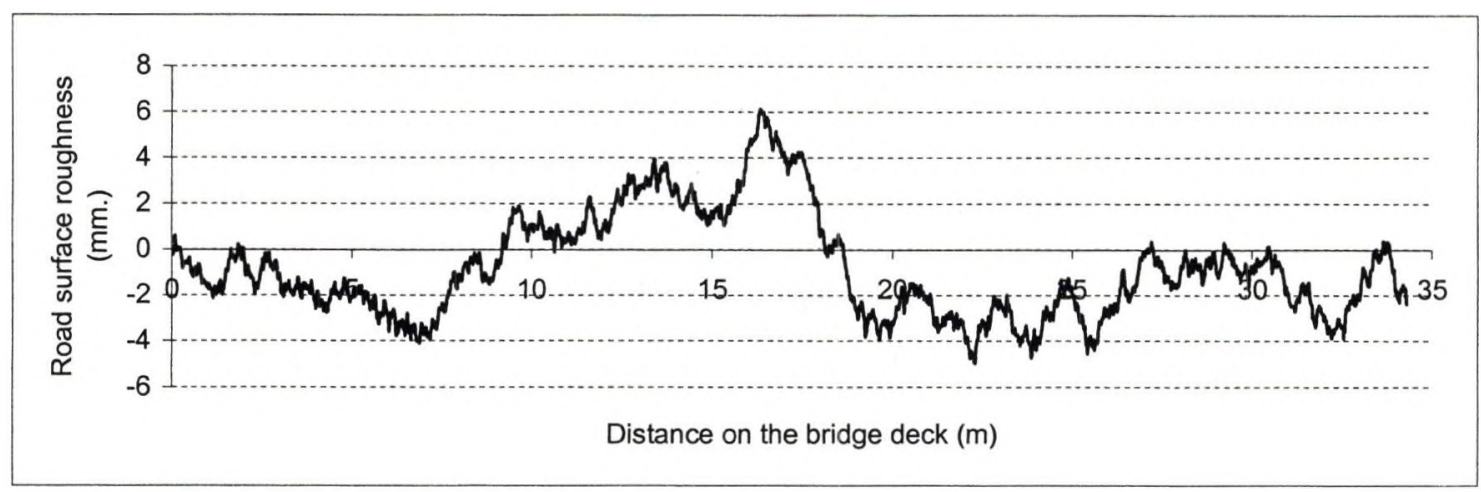

Figure 35: Road surface roughness of a very good road for the T-beam bridge deck.

Figure 36 shows the comparison of the PSD of the road surface roughness generated in MATLAB with the PSD given by C. J. Dodds and J. D. Robson [3], we can see from the comparison that the PSD of the road surface roughness is in well agreement with the PSD given by C. J. Dodds and J. D. Robson [3]. 


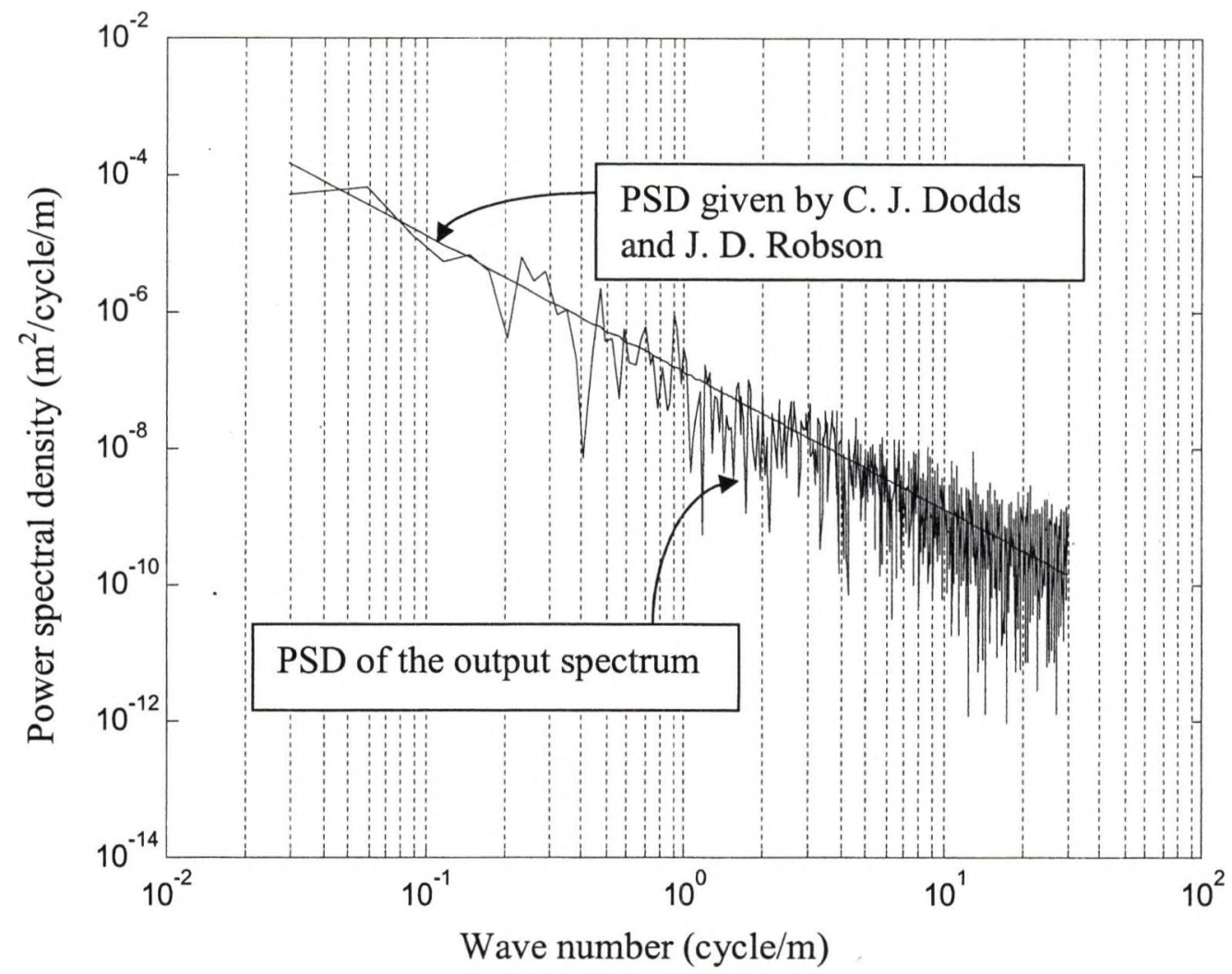

Figure 36: Comparison of the PSD of the road surface roughness generated in MATLAB with the PSD given by C. J. Dodds and J. D. Robson [3].

VII-b: Suspension and Tire Forces of an AASHTO HS20-44 Truck.

Road surface roughness shown in Figure 35 is used to calculate the increased load with an AASHTO HS20-44 truck [1] shown in Figures 9 and 10 traveling on the T-beam bridge deck at different speeds. Suspension force and tire force histories for steer, tractor and trailer axles are shown in Figures C.1 to C.22 in Appendix-C. The maximum suspension and tire forces for an AASHTO HS20-44 truck [1] at different speeds are summarized in Tables 20 and 21. From the Tables 20 and 21, for a HS20-44 truck traveling on a T-beam bridge deck with a very good road surface, we can see that the impact on suspension force is in the range of $19.14 \%$ to $38.58 \%$ of the static load for the 
steer axle and is reaching as much as $65.52 \%$ for the tractor axle, where as the maximum impact for the trailer axle is $50.82 \%$.

The impact for tire force is lesser than that for the suspension force. For the steer axle the impact is in the range of $14.76 \%$ to $29.57 \%$, the maximum impact for the tractor axle is $51.74 \%$ where as the maximum impact for trailer is $24.72 \%$ of the static load.

Table 20: Comparison of suspension forces at different speeds for HS20-44 truck on a Tbeam bridge deck.

\begin{tabular}{cccccccccc}
\hline $\begin{array}{c}\text { Speed } \\
\text { (mph) }\end{array}$ & $\begin{array}{c}\text { Static } \\
\text { (Kips) }\end{array}$ & $\begin{array}{c}\text { Fsy1 } \\
\text { (Kips) }\end{array}$ & \%Impact & $\begin{array}{c}\text { Static } \\
\text { (Kips) }\end{array}$ & $\begin{array}{c}\text { Fsy3 } \\
\text { (Kips) }\end{array}$ & \%lmpact & $\begin{array}{c}\text { Static } \\
\text { (Kips) }\end{array}$ & $\begin{array}{c}\text { Fsy5 } \\
\text { (Kips) }\end{array}$ & \%Impact \\
\hline 40 & 2.9119 & 3.4786 & 19.46 & 14.1780 & 22.4868 & 58.60 & 14.5579 & 20.6108 & 45.37 \\
45 & 2.9119 & 3.6736 & 26.16 & 14.1780 & 22.6332 & 59.64 & 14.5579 & 20.1593 & 42.19 \\
50 & 2.9119 & 3.5294 & 21.21 & 14.1780 & 22.2406 & 56.87 & 14.5579 & 19.9298 & 40.57 \\
55 & 2.9119 & 3.4692 & 19.14 & 14.1780 & 21.7862 & 53.66 & 14.5579 & 19.5557 & 37.93 \\
60 & 2.9119 & 3.5845 & 23.10 & 14.1780 & 22.0677 & 55.65 & 14.5579 & 21.3838 & 50.82 \\
65 & 2.9119 & 3.4699 & 19.16 & 14.1780 & 22.4452 & 58.31 & 14.5579 & 20.0683 & 41.55 \\
70 & 2.9119 & 3.5047 & 20.36 & 14.1780 & 22.8328 & 61.04 & 14.5579 & 20.1339 & 42.01 \\
75 & 2.9119 & 3.8023 & 30.58 & 14.1780 & 22.5749 & 59.22 & 14.5579 & 20.1479 & 42.11 \\
80 & 2.9119 & 3.7685 & 29.42 & 14.1780 & 22.0939 & 55.83 & 14.5579 & 20.7978 & 46.69 \\
85 & 2.9119 & 4.0352 & 38.58 & 14.1780 & 23.467 & 65.52 & 14.5579 & 20.3684 & 43.66 \\
90 & 2.9119 & 3.8211 & 31.22 & 14.1780 & 22.6275 & 59.60 & 14.5579 & 21.2594 & 49.95 \\
\hline
\end{tabular}

Note: Fsy2, Fsy4, and Fsy6 are same as Fsy1, Fsy3, and Fsy5 respectively.

Table 21: Comparison of tire forces at different speeds for HS20-44 truck on a T-beam bridge deck.

\begin{tabular}{cccccccccc}
\hline $\begin{array}{c}\text { Speed } \\
\text { (mph) }\end{array}$ & $\begin{array}{c}\text { Static } \\
\text { (Kips) }\end{array}$ & $\begin{array}{c}\text { Fty1 } \\
\text { (Kips) }\end{array}$ & \%lmpact & $\begin{array}{c}\text { Static } \\
\text { (Kips) }\end{array}$ & $\begin{array}{c}\text { Fty3 } \\
\text { (Kips) }\end{array}$ & \%lmpact & $\begin{array}{c}\text { Static } \\
\text { (Kips) }\end{array}$ & $\begin{array}{c}\text { Fty5 } \\
\text { (Kips) }\end{array}$ & \%Impact \\
\hline 40 & 3.99 & 4.6745 & 17.16 & 15.97 & 21.5269 & 34.80 & 15.99 & 19.9434 & 24.72 \\
45 & 3.99 & 4.7072 & 17.98 & 15.97 & 24.2326 & 51.74 & 15.99 & 19.8154 & 23.92 \\
50 & 3.99 & 4.7398 & 18.79 & 15.97 & 22.2853 & 39.54 & 15.99 & 19.2026 & 20.09 \\
55 & 3.99 & 4.5789 & 14.76 & 15.97 & 21.7315 & 36.08 & 15.99 & 18.8615 & 17.96 \\
60 & 3.99 & 4.8173 & 20.73 & 15.97 & 22.2798 & 39.51 & 15.99 & 19.2308 & 20.27 \\
65 & 3.99 & 4.7157 & 18.19 & 15.97 & 22.6370 & 41.75 & 15.99 & 19.6400 & 22.83 \\
70 & 3.99 & 4.7860 & 19.95 & 15.97 & 22.8174 & 42.88 & 15.99 & 19.8072 & 23.87 \\
75 & 3.99 & 4.8639 & 21.90 & 15.97 & 23.9411 & 49.91 & 15.99 & 19.3369 & 20.93 \\
80 & 3.99 & 4.8701 & 22.06 & 15.97 & 23.0975 & 44.63 & 15.99 & 19.5071 & 22.00 \\
85 & 3.99 & 5.0782 & 27.27 & 15.97 & 23.3027 & 45.92 & 15.99 & 19.8858 & 24.36 \\
90 & 3.99 & 5.1698 & 29.57 & 15.97 & 23.8396 & 49.28 & 15.99 & 19.4418 & 21.59 \\
\hline
\end{tabular}

Note: Fty2, Fty4, and Fty6 are same as Fty1, Fty3, and Fty5 respectively. 
The increased load from Table 21, which has a cumulative effect of road surface roughness and vehicle dynamics, is considered for the analysis first by using the orthotropic plate theory as explained in Chapter V and secondly by the finite element analysis as explained in Chapter VI.

VII-c: Analysis of the T-beam Bridge Deck by using the Orthotropic Plate Theory. Tire forces from Table 21 are multiplied by 2 to get the total axle load for the steer axle, trailer axle, and tractor axle of the truck. Table 22 shows the total axle loads at different speeds.

Table 22: Total axle load of an AASHTO HS20-44 truck on a T-beam bridge deck.

\begin{tabular}{|c|c|c|c|}
\hline \multirow{2}{*}{$\begin{array}{l}\text { Speed } \\
\text { (mph) }\end{array}$} & \multicolumn{3}{|c|}{$\begin{array}{c}\text { Total axle load } \\
\text { (lb.) }\end{array}$} \\
\hline & $\begin{array}{l}\text { Steer } \\
\text { axle }\end{array}$ & $\begin{array}{c}\text { Tractor } \\
\text { axle }\end{array}$ & $\begin{array}{c}\text { Trailer } \\
\text { axle }\end{array}$ \\
\hline 40 & 9349 & 43053.8 & 39886.8 \\
\hline 45 & 9414.4 & 48465.2 & 39630.8 \\
\hline 50 & 9479.6 & 44570.6 & 38405.2 \\
\hline 55 & 9157.8 & 43463 & 37723 \\
\hline 60 & 9634.6 & 44559.6 & 38461.6 \\
\hline 65 & 9431.4 & 45274 & 39280 \\
\hline 70 & 9572 & 45634.8 & 39614.4 \\
\hline 75 & 9727.8 & 47882.2 & 38673.8 \\
\hline 80 & 9740.2 & 46195 & 39014.2 \\
\hline 85 & 10156.4 & 46605.4 & 39771.6 \\
\hline 90 & 10339.6 & 47679.2 & 38883.6 \\
\hline
\end{tabular}

A T-beam bridge deck shown in Figure 32 is used for the analysis. Dimensions and the physical properties of the plate are $a=112.5 \mathrm{ft}, b=42.66 \mathrm{ft}, h=0.58 \mathrm{ft}$, $\rho=4.658$ slugs $/ f t^{3}, v_{x y}=0.2, D_{x}=2.3285 \times 10^{9} l b . f t, D_{y}=0.2575 \times 10^{9} l b . f t$, and $2 H=0.0342 \times 10^{9} \mathrm{lb} . f t$. 
Figures C.23 to C. 33 in Appendix-C show the time history plot of the vertical displacements at the center of the T-beam bridge deck under an AASHTO HS20-44 truck at different speeds.

Table 23 summarizes the maximum vertical displacement at the center of the Tbeam bridge deck (node \# 2901) at different speeds of the truck.

Table 23: Maximum vertical displacements at the center of the T-beam bridge deck under an AASHTO HS20-44 truck moving with different speeds by the orthotropic plate theory.

\begin{tabular}{cccc}
\hline $\begin{array}{c}\text { Speed } \\
\text { (mph) }\end{array}$ & $\begin{array}{c}\text { Vertical } \\
\text { displacementdisplacement } \\
\text { (in.) }\end{array}$ & $\begin{array}{c}\text { Static } \\
\text { (in.) }\end{array}$ & $\begin{array}{c}\text { Impact } \\
\text { (\%) }\end{array}$ \\
\hline 40 & 0.2988 & 0.2184 & 36.81 \\
45 & 0.3144 & 0.2184 & 43.96 \\
50 & 0.3048 & 0.2184 & 39.56 \\
55 & 0.3012 & 0.2184 & 37.91 \\
60 & 0.3060 & 0.2184 & 40.11 \\
65 & 0.3036 & 0.2184 & 39.01 \\
70 & 0.2988 & 0.2184 & 36.81 \\
75 & 0.3024 & 0.2184 & 38.46 \\
80 & 0.3012 & 0.2184 & 37.91 \\
85 & 0.3084 & 0.2184 & 41.21 \\
90 & 0.3120 & 0.2184 & 42.86 \\
\hline
\end{tabular}

Figure 37 shows the comparison of the vertical displacement under a moving truck load with the static displacement. Static displacement in Table 23 and Figure 37 is calculated by assuming the AASHTO HS20-44 truck moving at a speed of $5 \mathrm{mph}$. 


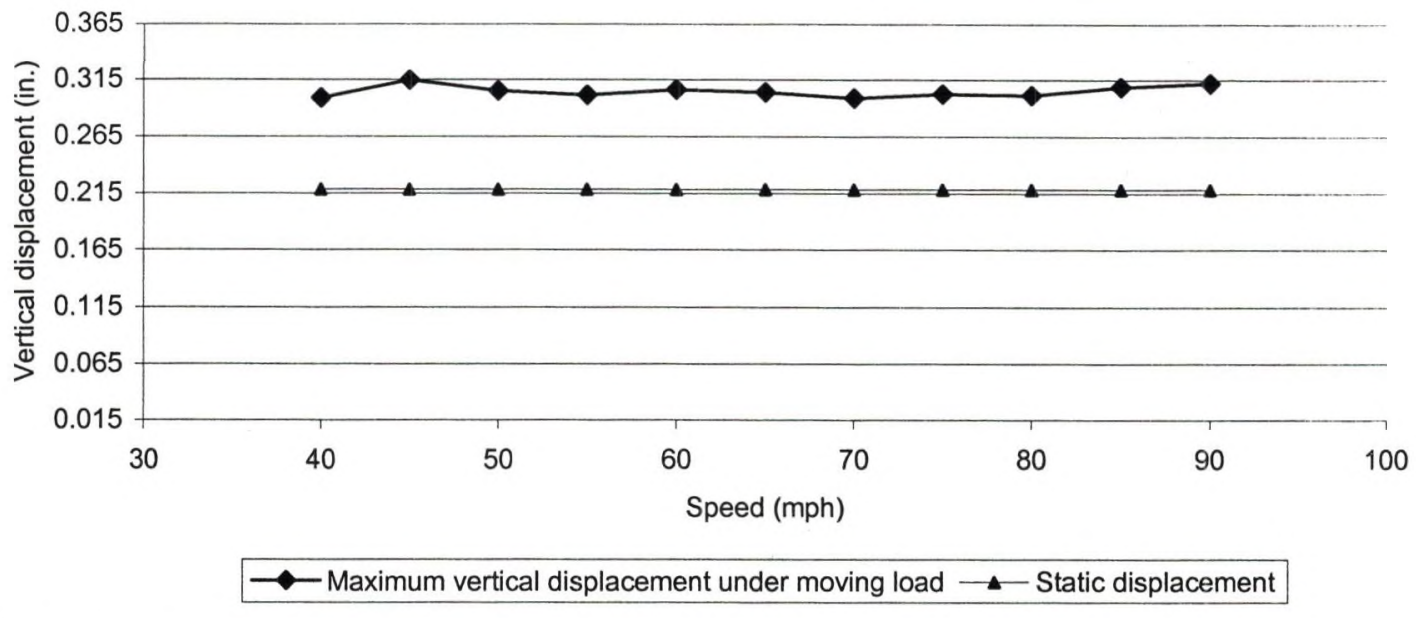

Figure 37: Comparison of the vertical displacement under a moving AASHTO HS20-44 truck load with the static displacement by the orthotropic plate theory.

From Table 23 and Figure 37 we can see that the impact on the maximum vertical displacement at the center of the plate by using the orthotropic plate theory is in the range of $36.81 \%$ to $43.96 \%$ whereas the impact given by AASHTO is $21.05 \%$. This tells us that the impact factor formula given by AASHTO underestimates the dynamic effect of the bridge under moving loads. This might be because of the inability of the impact factor formula given by AASHTO, which is a function of the span length of the bridge deck, to take into account the effect of road surface roughness, vehicle dynamics, vehicle weight, and vehicle speed.

VII-d: Analysis of the T-beam Bridge Deck by using the Finite Element Analysis Method.

The T-beam bridge deck shown in Figure 32 is then modeled in the finite element analysis package NISA. 3D beam elements are used to model the longitudinal and transverse girders of the bridge, and 3D shell elements are used to model the slab of the bridge deck. Increased load from the road surface roughness and vehicle dynamics given 
in Table 22 is used to simulate the AASHTO HS20-44 truck [1] load moving at different speeds on the centerline of the bridge deck. Figures C. 34 to C. 44 in Appendix-C show the time history of the vertical displacement at the center of the bridge deck at different speeds. Table 24 summarizes the maximum vertical displacement at the center of the Tbeam bridge deck (node \# 2901) at different speeds of the truck by the finite element analysis method.

Table 24: Maximum vertical displacements at the center of the T-beam bridge deck under an AASHTO HS20-44 truck moving with different speeds by FEM.

\begin{tabular}{cccc}
\hline $\begin{array}{c}\text { Speed } \\
\text { (mph) }\end{array}$ & $\begin{array}{c}\text { Vertical } \\
\text { displacementdisplacement } \\
\text { (in.) }\end{array}$ & $\begin{array}{c}\text { Static } \\
\text { (in.) }\end{array}$ & $\begin{array}{c}\text { Impact } \\
\text { (\%) }\end{array}$ \\
\hline 40 & 0.346 & 0.2557 & 35.31 \\
45 & 0.351 & 0.2557 & 37.27 \\
50 & 0.334 & 0.2557 & 30.62 \\
55 & 0.333 & 0.2557 & 30.23 \\
60 & 0.343 & 0.2557 & 34.14 \\
65 & 0.346 & 0.2557 & 35.31 \\
70 & 0.346 & 0.2557 & 35.31 \\
75 & 0.352 & 0.2557 & 37.66 \\
80 & 0.358 & 0.2557 & 40.01 \\
85 & 0.375 & 0.2557 & 46.66 \\
90 & 0.390 & 0.2557 & 52.52 \\
\hline
\end{tabular}

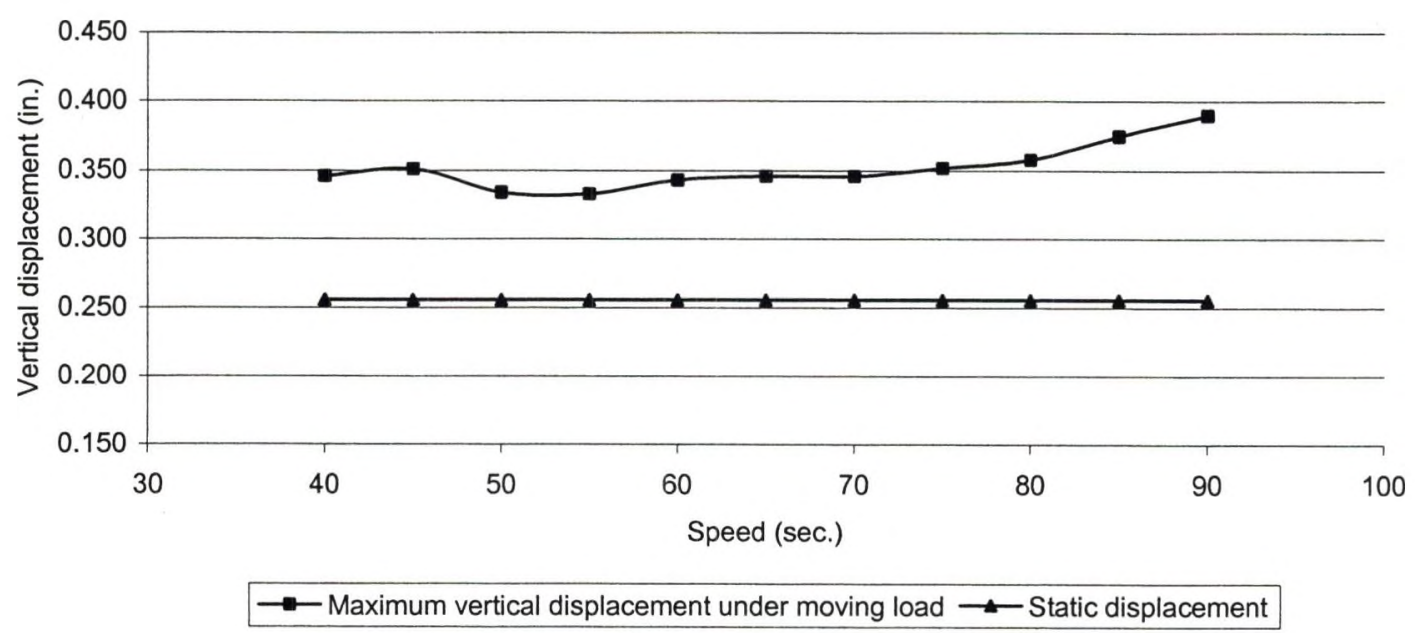

Figure 38: Comparison of the vertical displacement under a moving AASHTO HS20-44 truck load with the static displacement by FEM. 
Figure 38 shows the comparison of the vertical displacement under a moving truck load with the static displacement. Static displacement in Table 24 and Figure 38 is calculated by assuming the AASHTO HS20-44 truck moving at a speed of $5 \mathrm{mph}$.

From Table 24 and Figure 38 we can see that the impact on the maximum vertical displacement at the center of the plate by using the finite element analysis method is in the range of $30.23 \%$ to $52.52 \%$ whereas the impact given by AASHTO is $21.05 \%$. Impact calculated by the FEM confirms that the impact factor formula given by AASHTO underestimate the dynamic effect of bridges under moving loads.

Table 25 and Figure 39 show the comparison of the impact calculated on the maximum vertical displacement at the center of the plate by using the orthotropic plate theory and by using the finite element analysis method.

Table 25: Comparison of maximum vertical displacements at the center of the T-beam bridge deck under an AASHTO HS20-44 truck moving with different speeds by the orthotropic plate theory and by the finite element analysis method.

\begin{tabular}{ccc}
\hline \multirow{2}{*}{$\begin{array}{c}\text { Speed } \\
(\mathrm{mph})\end{array}$} & \multicolumn{2}{c}{$\begin{array}{c}\text { Impact } \\
(\%)\end{array}$} \\
\cline { 2 - 3 } & Orthotropic plate theory & Finite element method \\
\hline 40 & 36.81 & 35.31 \\
45 & 43.96 & 37.27 \\
50 & 39.56 & 30.62 \\
55 & 37.91 & 30.23 \\
60 & 40.11 & 34.14 \\
65 & 39.01 & 35.31 \\
70 & 36.81 & 35.31 \\
75 & 38.46 & 37.66 \\
80 & 37.91 & 40.01 \\
85 & 41.21 & 46.66 \\
90 & 42.86 & 52.52 \\
\hline
\end{tabular}




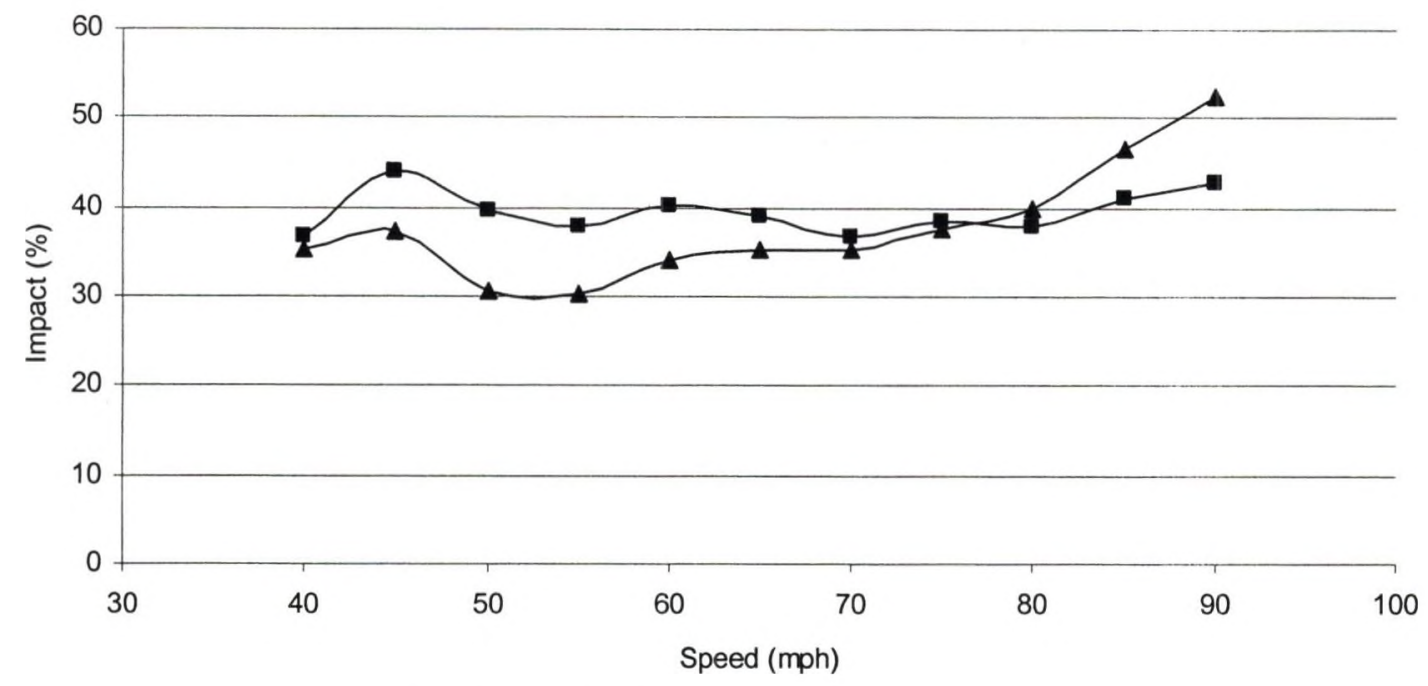

- Orthotropic plate theory $\multimap$ - Finite element analysis method

Figure 39: Comparison of maximum vertical displacements at the center of the T-beam bridge deck under an AASHTO HS20-44 truck moving with different speeds by the orthotropic plate theory and by the finite element analysis method.

From Table 25 and Figure 39 we can see that the impact calculated by the orthotropic plate theory is close to $40 \%$ for all the speeds and the impact calculated by the finite element analysis method is less as much as $30 \%$ for the slower speeds and increases to over $50 \%$ as the speed increases. 


\section{CHAPTER VIII}

\section{CONCLUSIONS AND RECOMMENDATIONS}

In this study the cumulative effect of the road surface roughness, vehicle dynamics, vehicle weight, and vehicle speed on the bridge dynamics is investigated in terms of the vertical displacement at the center of the bridge deck. Road surface roughness is generated by using the Power Spectral Density function given by C. J. Dodds and J. D. Robson [3]. A 12 DOF model of an AASHTO HS20-44 truck is developed. This truck model is assumed to be moving on a very good and good road surface at different speeds to see the effect of the road surface roughness and vehicle dynamics on the suspension and tire forces of the AASHTO HS20-44 truck.

Two methods are used in the study to analyze the bridges under moving loads, the orthotropic plate theory and the finite element analysis method. The increased load calculated above is simulated by using a Dirac-delta function for the analysis by the orthotropic plate theory, and by using the arrival time data and time function data for the analysis by the finite element analysis method.

Finally, a T-beam bridge is investigated to see the cumulative effect of the road surface roughness, vehicle dynamics, vehicle weight, and vehicle speed on the bridge dynamics in terms of the vertical displacement at the center of the T-beam bridge deck. The T-beam bridge deck in the consideration is assumed to be built on a motorway with a very good road condition. Road surface roughness generated is assumed 
to be the same along the width of the bridge deck. An AASHTO HS20-44 truck is considered to be moving along the centerline of the bridge deck with a constant speed. The truck is assumed to be moving only in X-direction and contact of the truck wheel with the deck is assumed to be at a single point.

Impact factors calculated for the suspension forces of an AASHTO HS20-44 truck on a very good surface indicate that the impact for the steer axle, tractor axle, and trailer axle is $44.71 \%, 73.31 \%$, and $51.52 \%$ respectively. Impact factors calculated for the tire forces of an AASHTO HS20-44 truck on a very good surface indicate that the impact for the steer axle, tractor axle, and trailer axle is $44.21 \%, 51.20 \%$, and $32.21 \%$ respectively. These impact factors increase noticeably for a good road condition.

The impact on the vertical displacement at the center of the plate under a single moving load calculated without considering the effect of road surface roughness and vehicle dynamics indicates that the impact is more when we consider a single load than the two loads moving along the centerline of the bridge. The impact is as much as $16.82 \%$ for a single load and as much as $9.65 \%$ for the two loads moving along the centerline of the bridge.

The forces calculated for an AASHTO HS20-44 truck on a very good road surface indicate that the impact for the tire forces is less than that for the suspension forces and is as much as $29.57 \%$ for the steer axle, $51.74 \%$ for the tractor axle, and $24.72 \%$ for the trailer axle. Finally, when we consider the cumulative effect of the road surface roughness, vehicle dynamics, vehicle weight, and vehicle speed, the impact calculated by the orthotropic plate theory is as much as $43.96 \%$ and that is calculated by the finite element analysis method is as much as $52.52 \%$. 
Impact calculated without considering the road surface roughness and vehicle dynamics under a single moving load is less than the impact calculated by using the impact formula given by AASHTO which is $21.05 \%$ and further decreases with the number of loads moving along the centerline of the bridge deck. Impact calculated by considering all the factors such as road surface roughness, vehicle dynamics, vehicle weight, and vehicle speed by both the methods is reasonably higher than that calculated by the impact formula given by AASHTO.

For the bridge deck under investigation the impact factor formula given by AASHTO underestimates the dynamic effect under the moving loads. This might be because of the inability of the impact factor formula given by AASHTO, which is a function of span length of the bridge deck, to take into account the effect of road surface roughness, vehicle dynamics, vehicle weight, and vehicle speeds. This suggest that, it is necessary to do the detailed dynamic analysis of the bridges by considering the road surface roughness, vehicle dynamics, vehicle weight and speed instead of just applying the dynamic load factor calculated by using the impact factor given by AASHTO to the static results. 

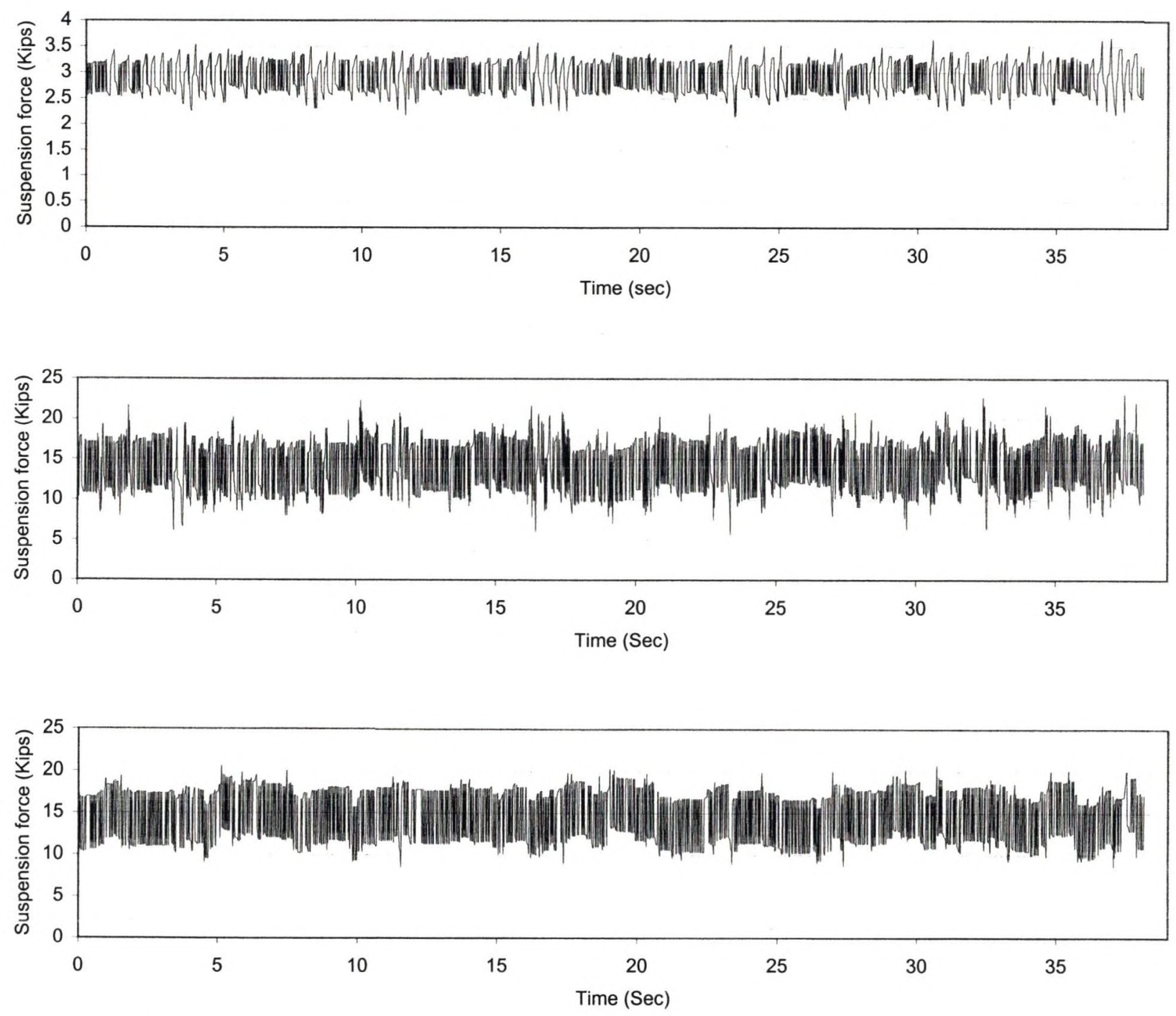

Figure A.1: Suspension force history of axles one, two, and three on a very good road surface at the speed of $15 \mathrm{mph}$ 

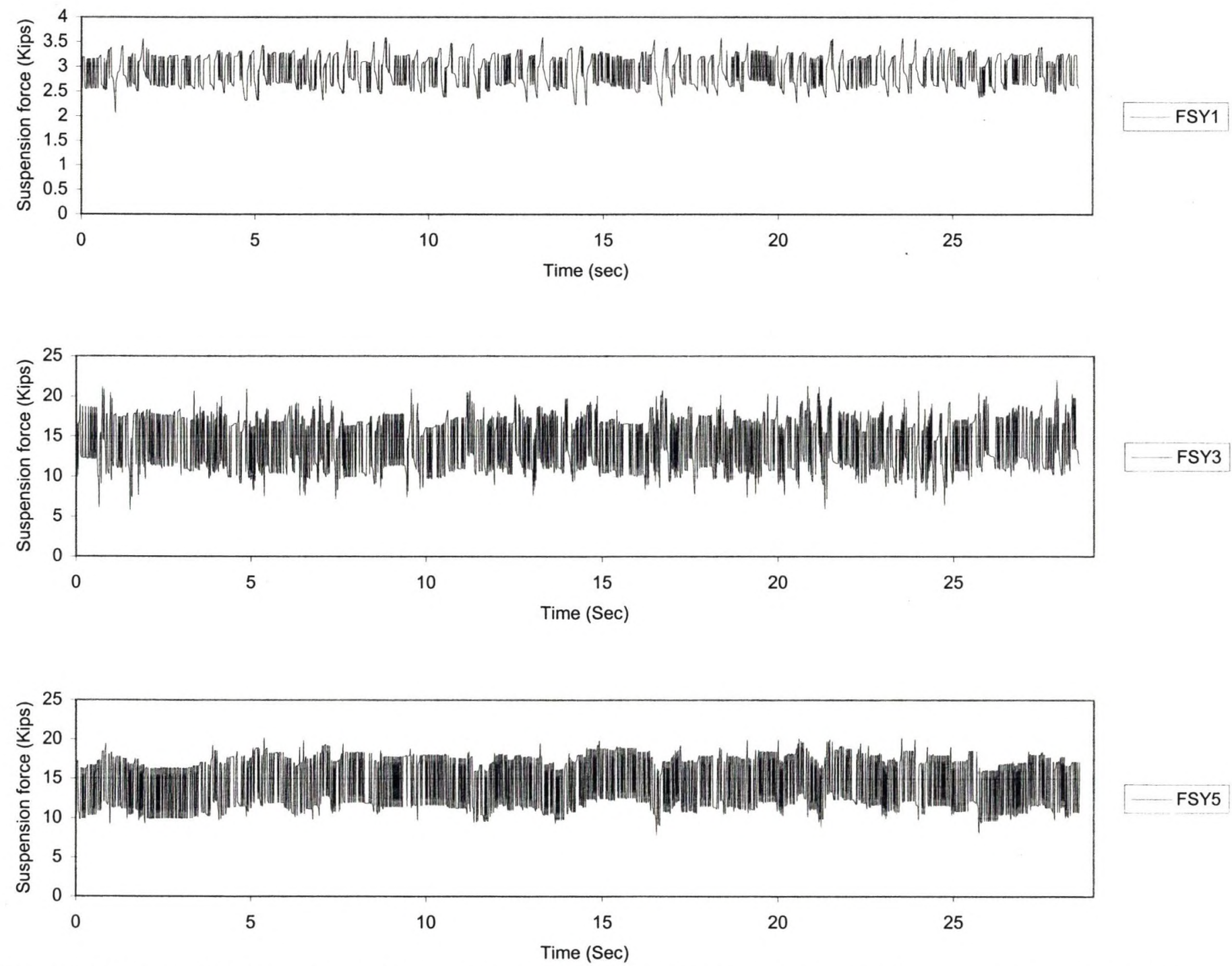

Figure A.2: Suspension force history of axles one, two, and three on a very good road surface at the speed of $20 \mathrm{mph}$ 

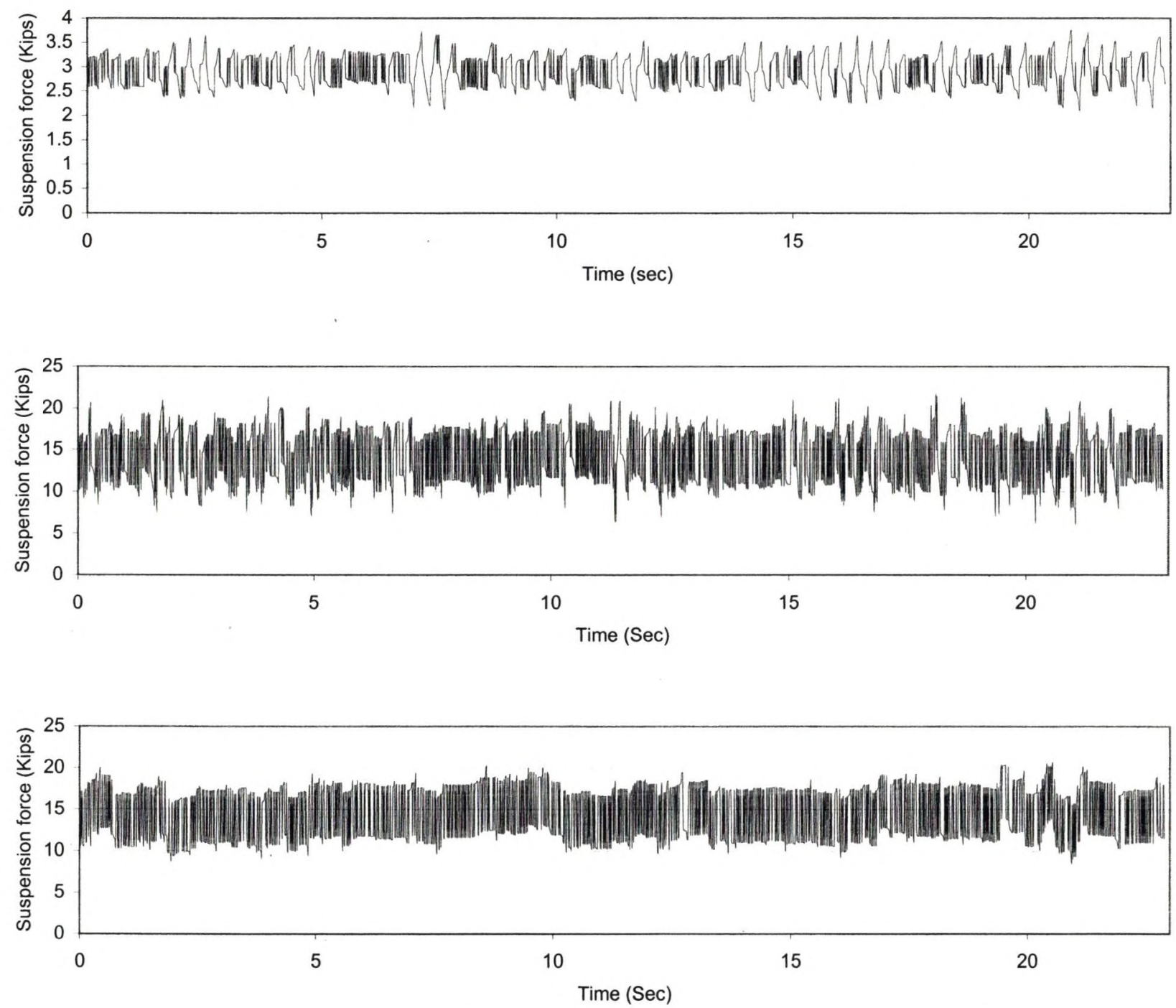

Figure A.3: Suspension force history of axles one, two, and three on a very good road surface at the speed of $25 \mathrm{mph}$ 

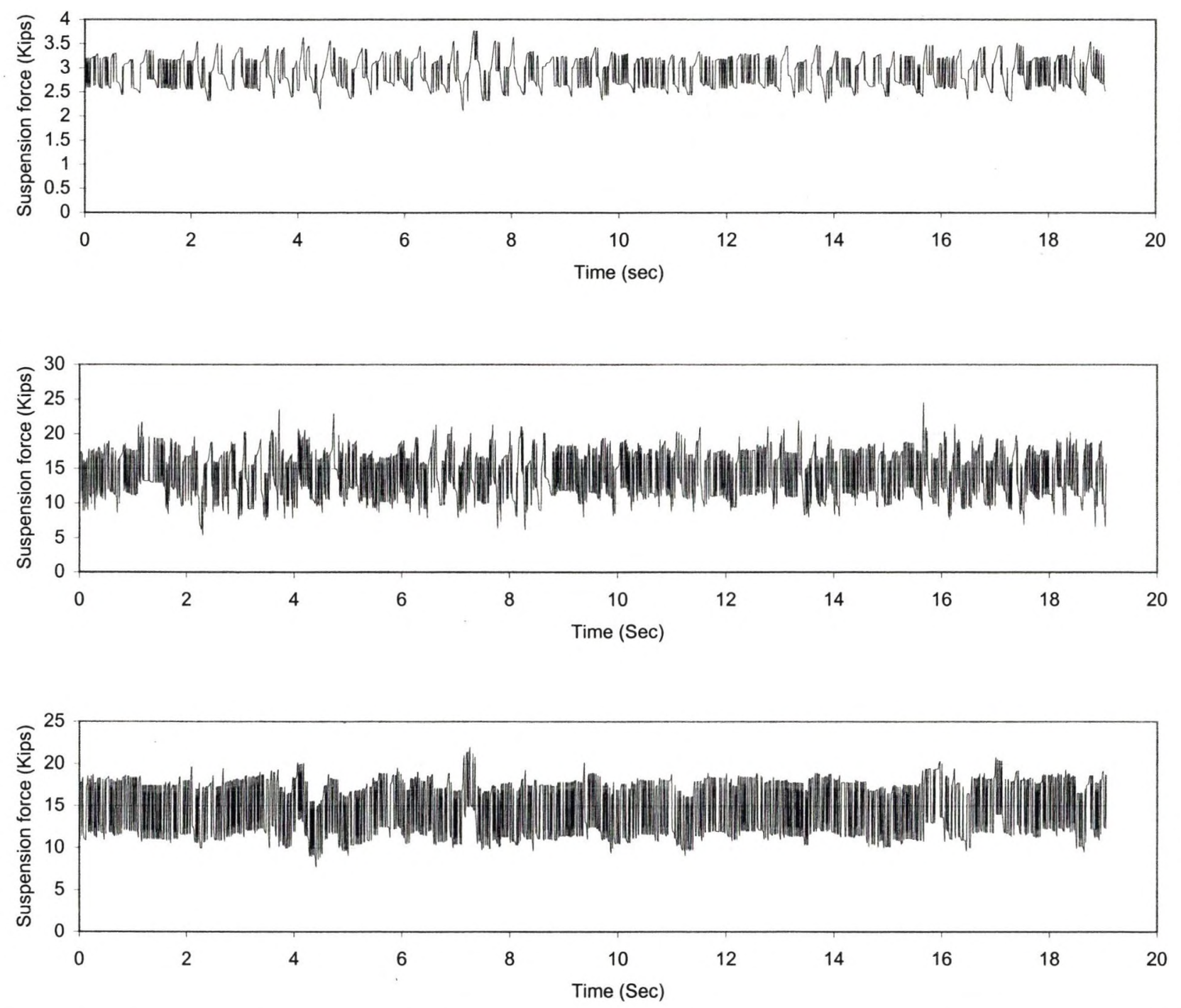

Figure A.4: Suspension force history of axles one, two, and three on a very good road surface at the speed of $30 \mathrm{mph}$ 

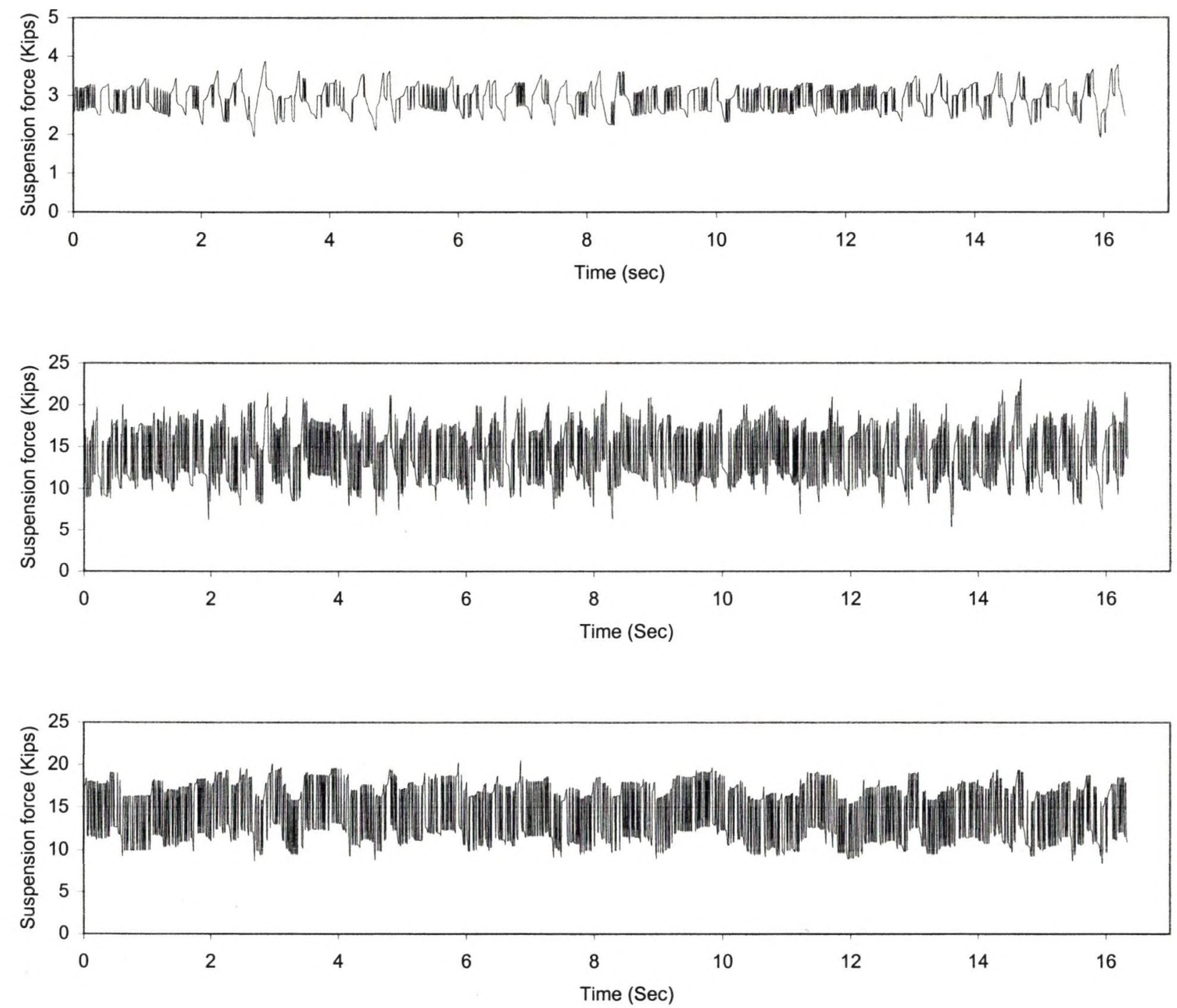

Figure A.5: Suspension force history of axles one, two, and three on a very good road surface at the speed of $35 \mathrm{mph}$ 

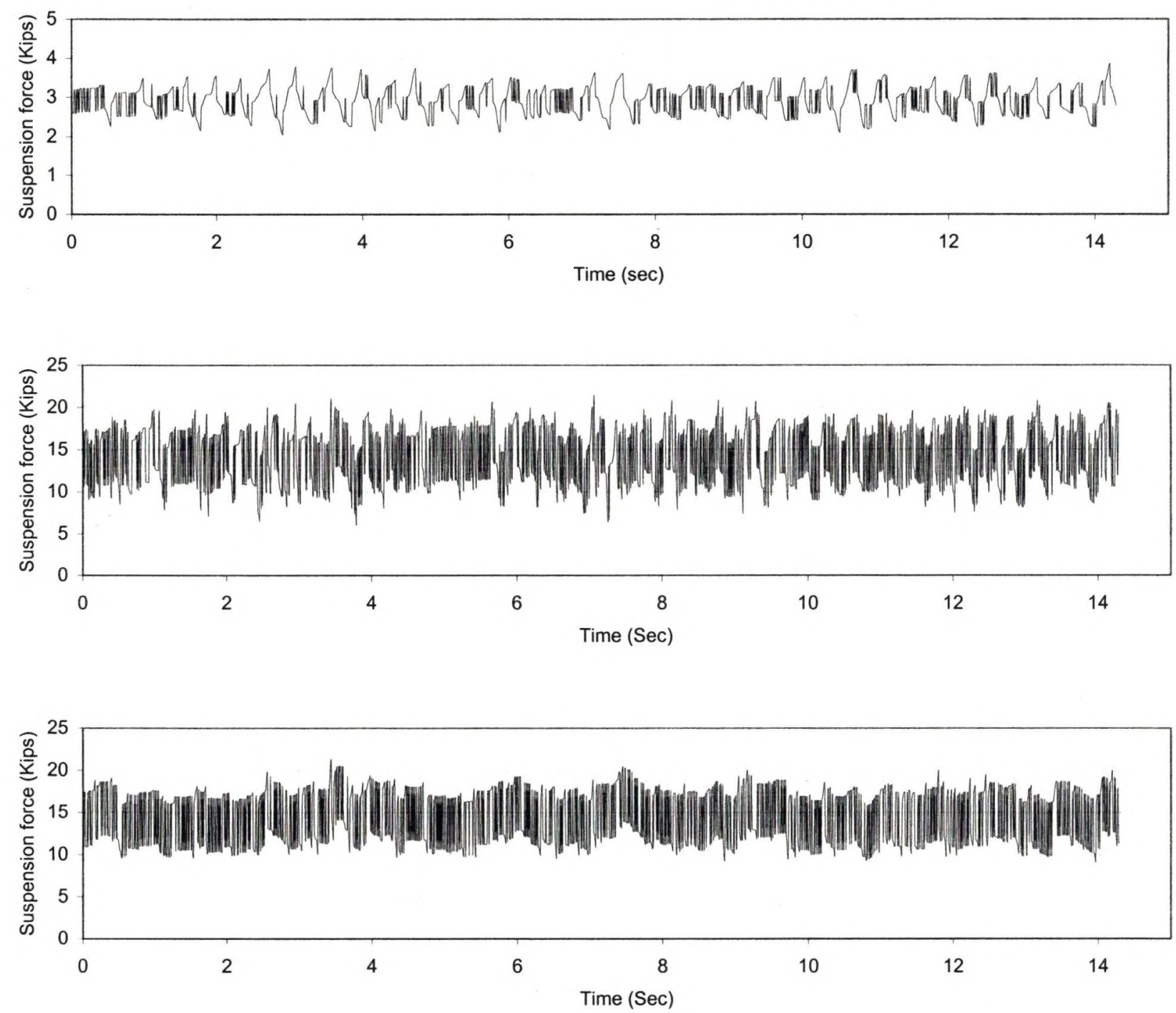

Figure A.6: Suspension force history of axles one, two, and three on a very good road surface at the speed of $40 \mathrm{mph}$ 

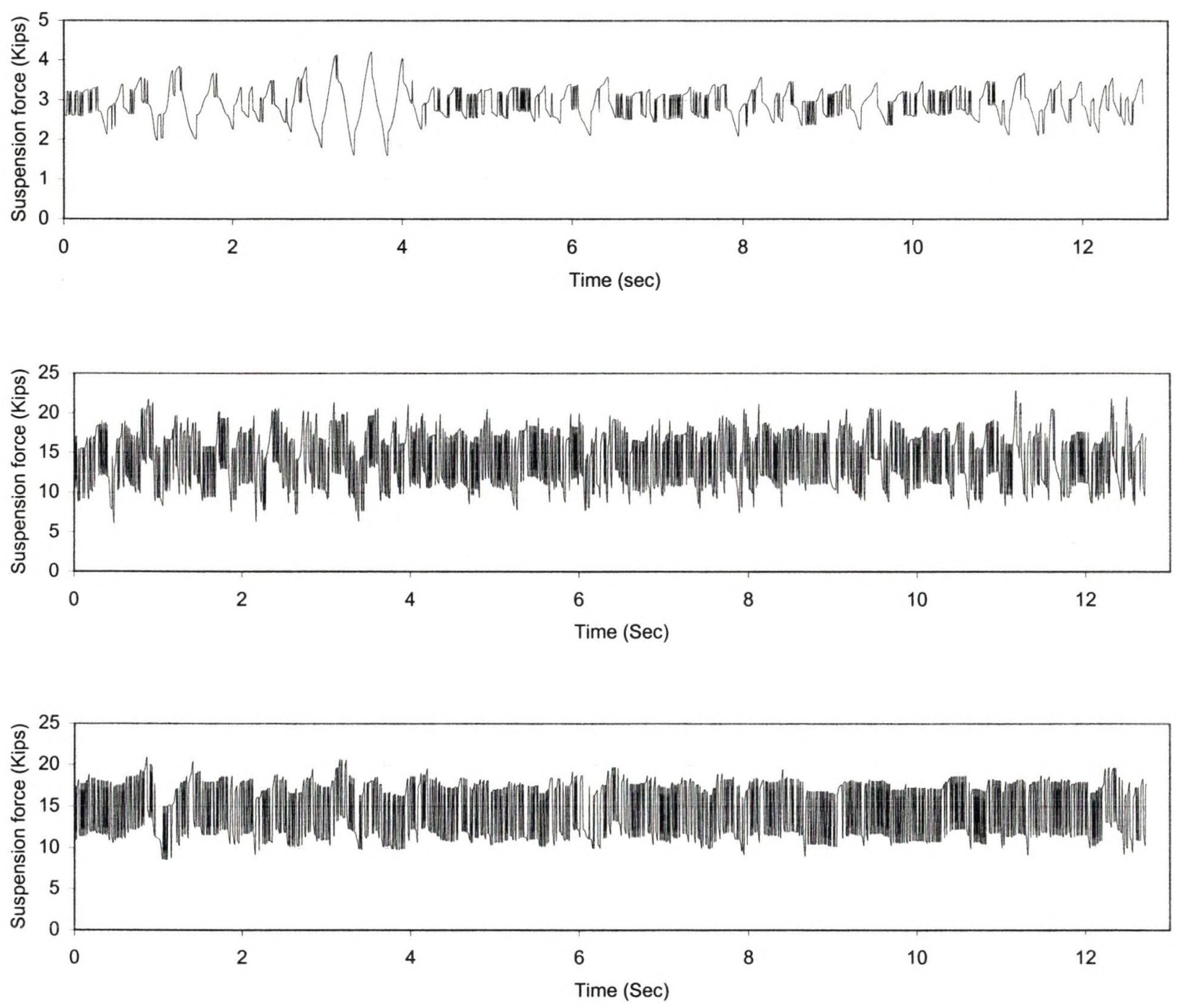

Figure A.7: Suspension force history of axles one, two, and three on a very good road surface at the speed of $45 \mathrm{mph}$ 

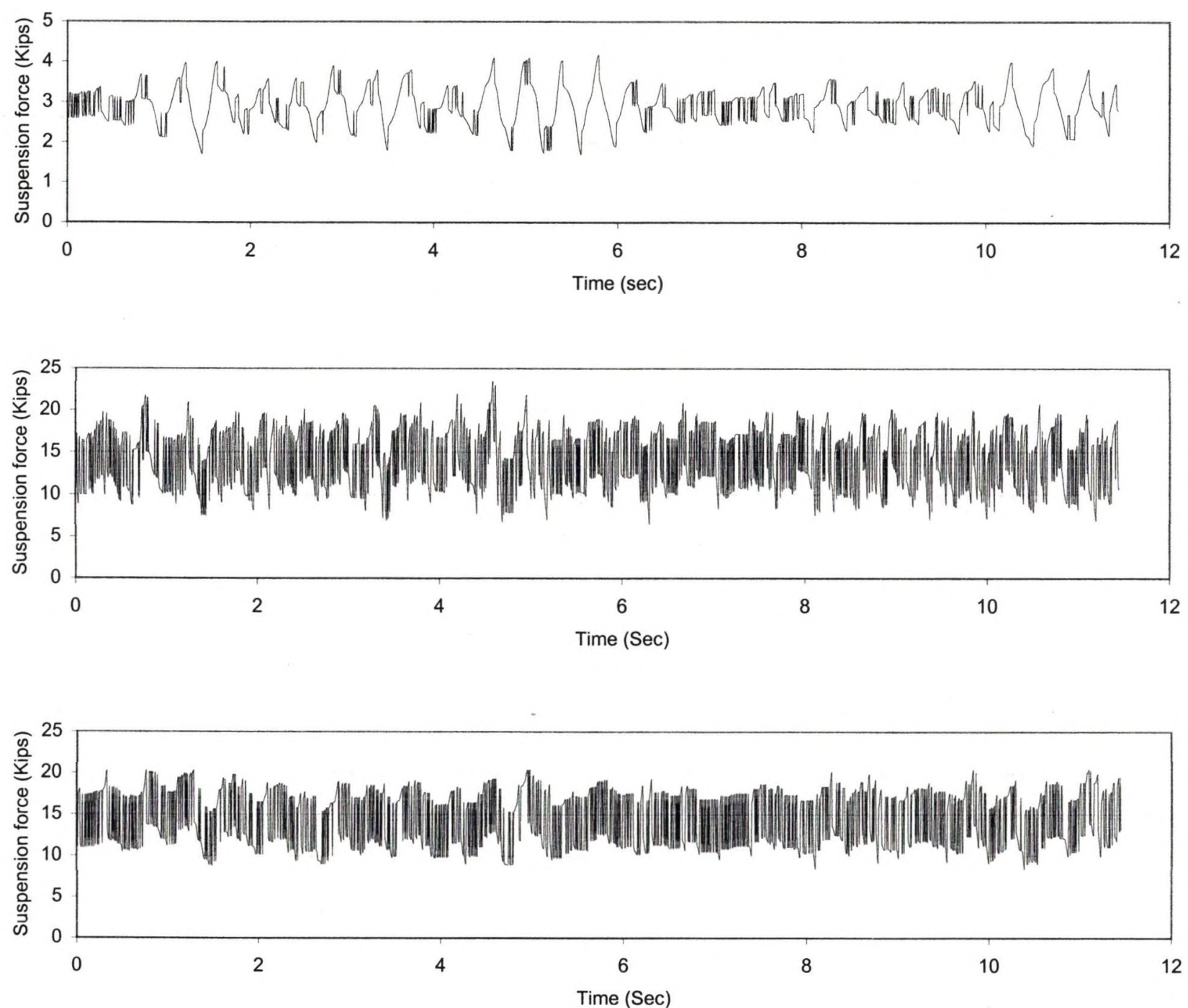

Figure A.8: Suspension force history of axles one, two, and three on a very good road surface at the speed of $50 \mathrm{mph}$ 

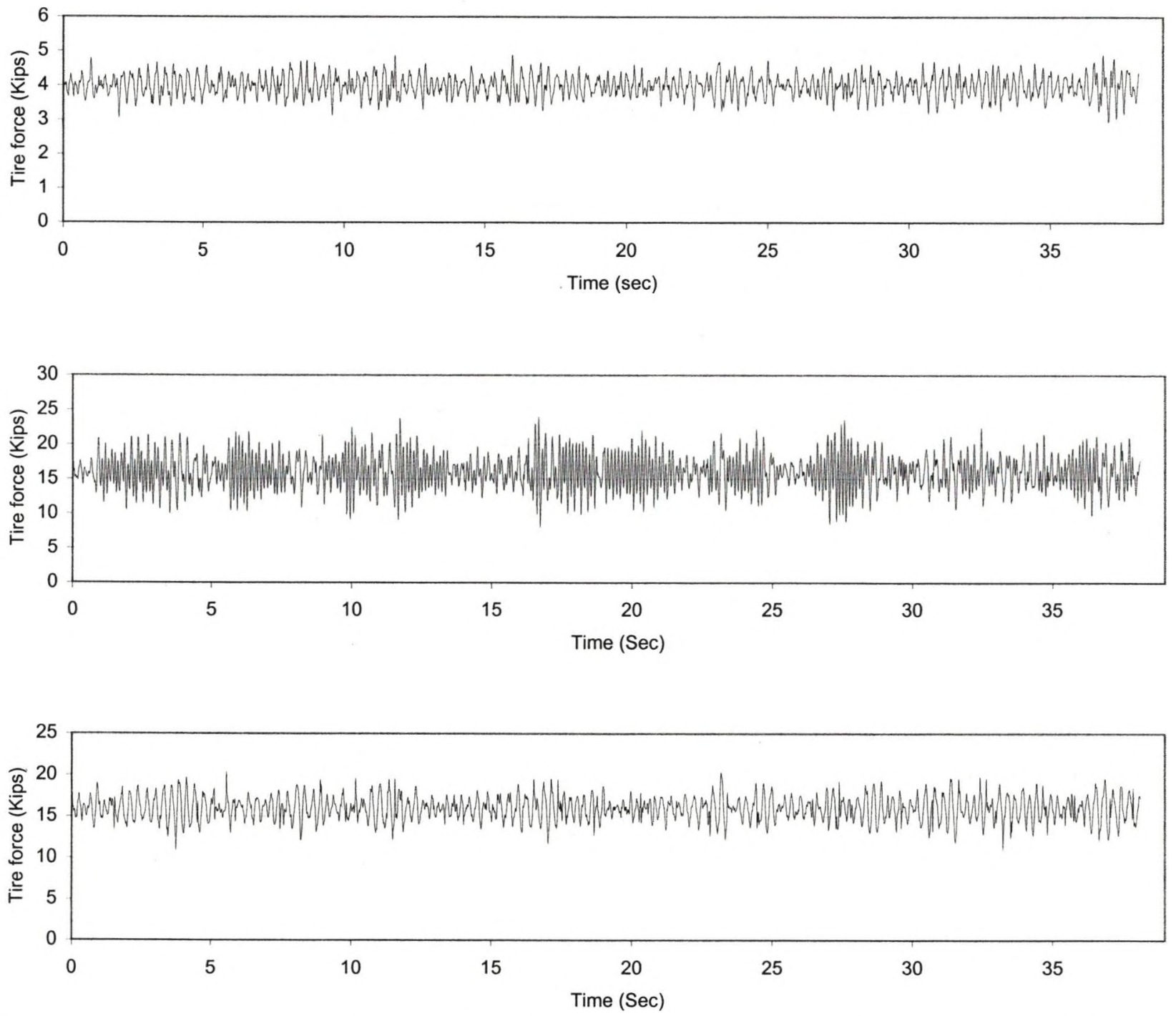

Figure A.9: Tire force history of axles one, two, and three on a very good road surface at the speed of $15 \mathrm{mph}$ 

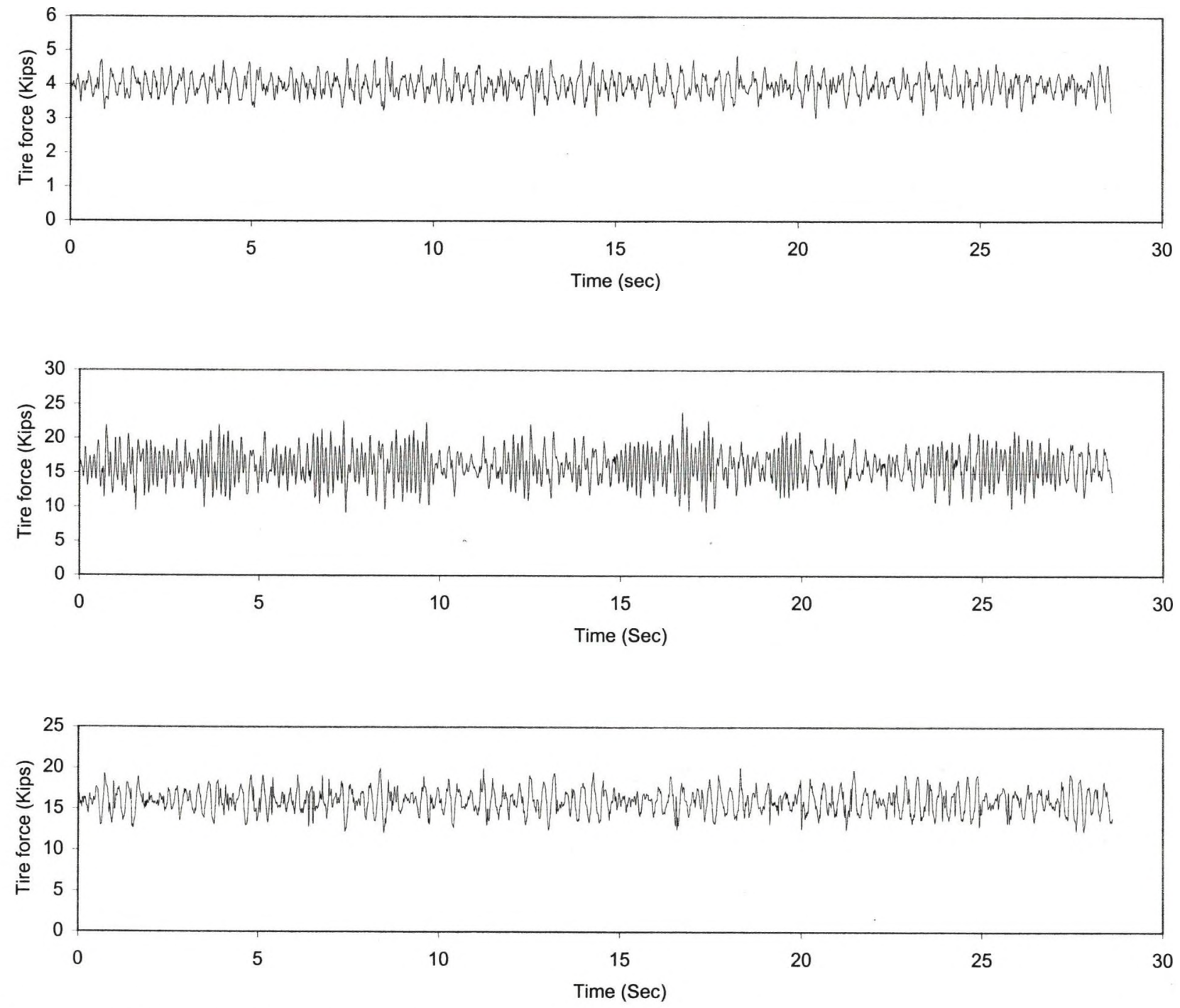

Figure A.10: Tire force history of axles one, two, and three on a very good road surface at the speed of $20 \mathrm{mph}$ 

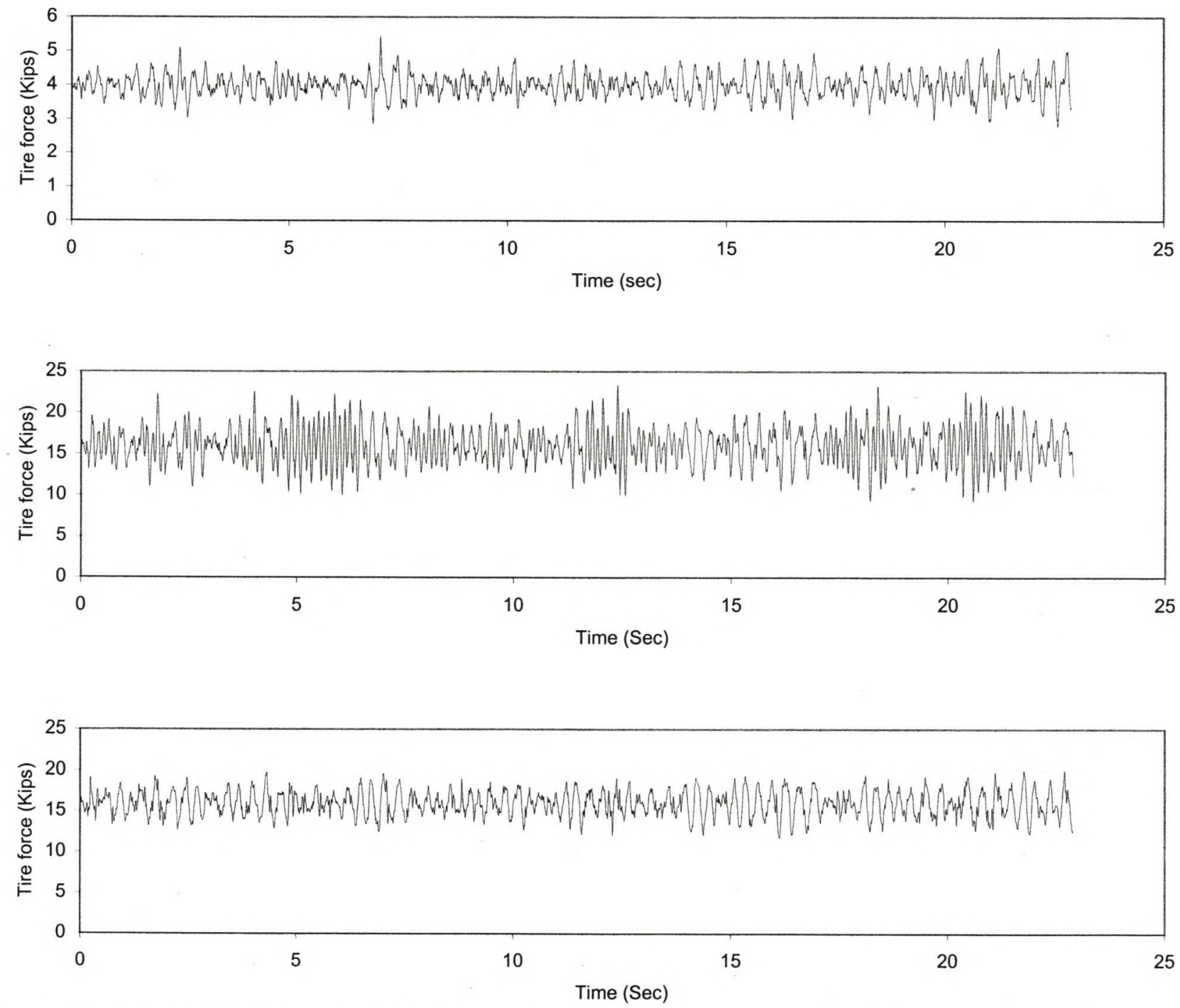

Figure A.11: Tire force history of axles one, two, and three on a very good road surface at the speed of $25 \mathrm{mph}$ 

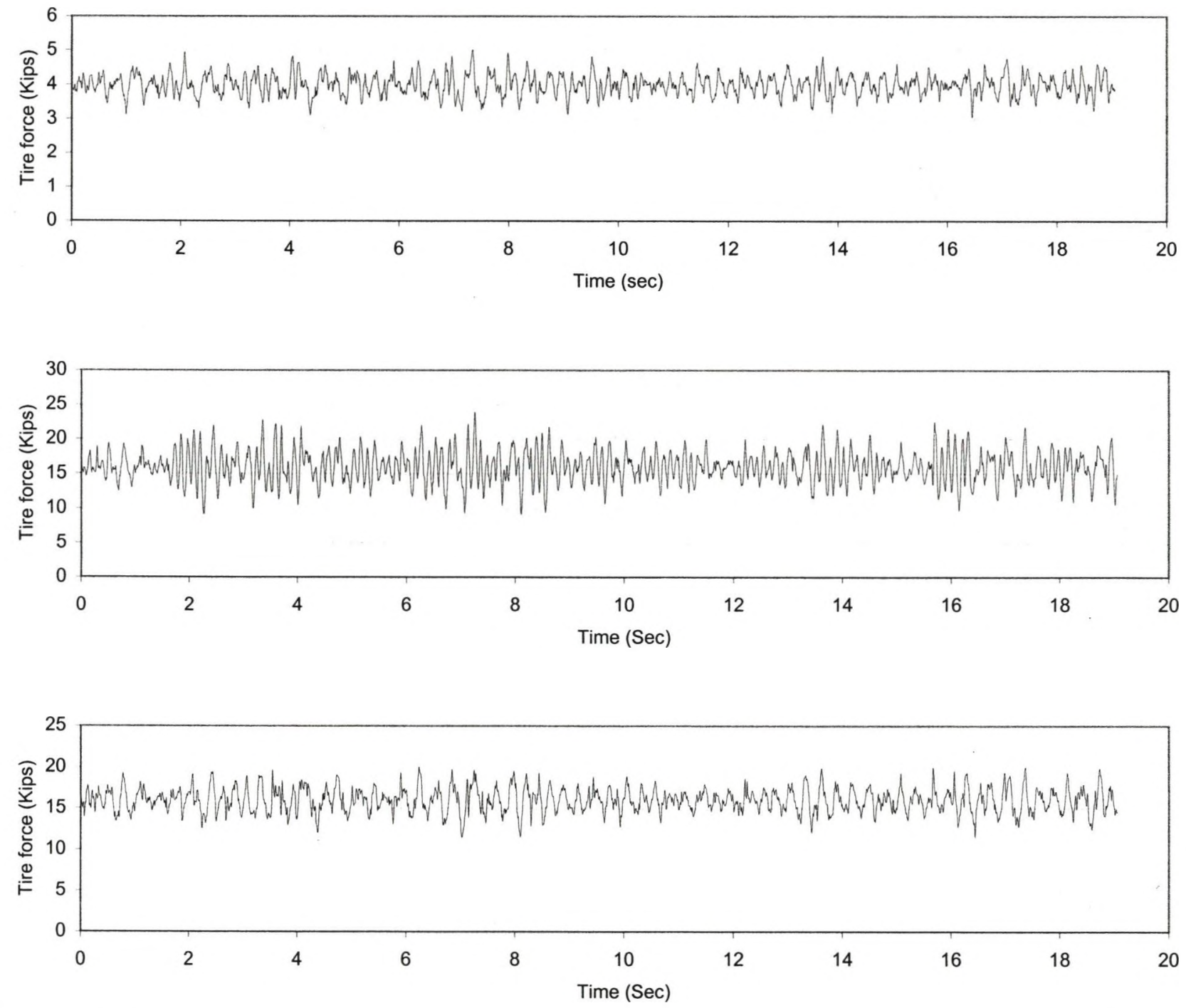

Figure A.12: Tire force history of axles one, two, and three on a very good road surface at the speed of $30 \mathrm{mph}$ 

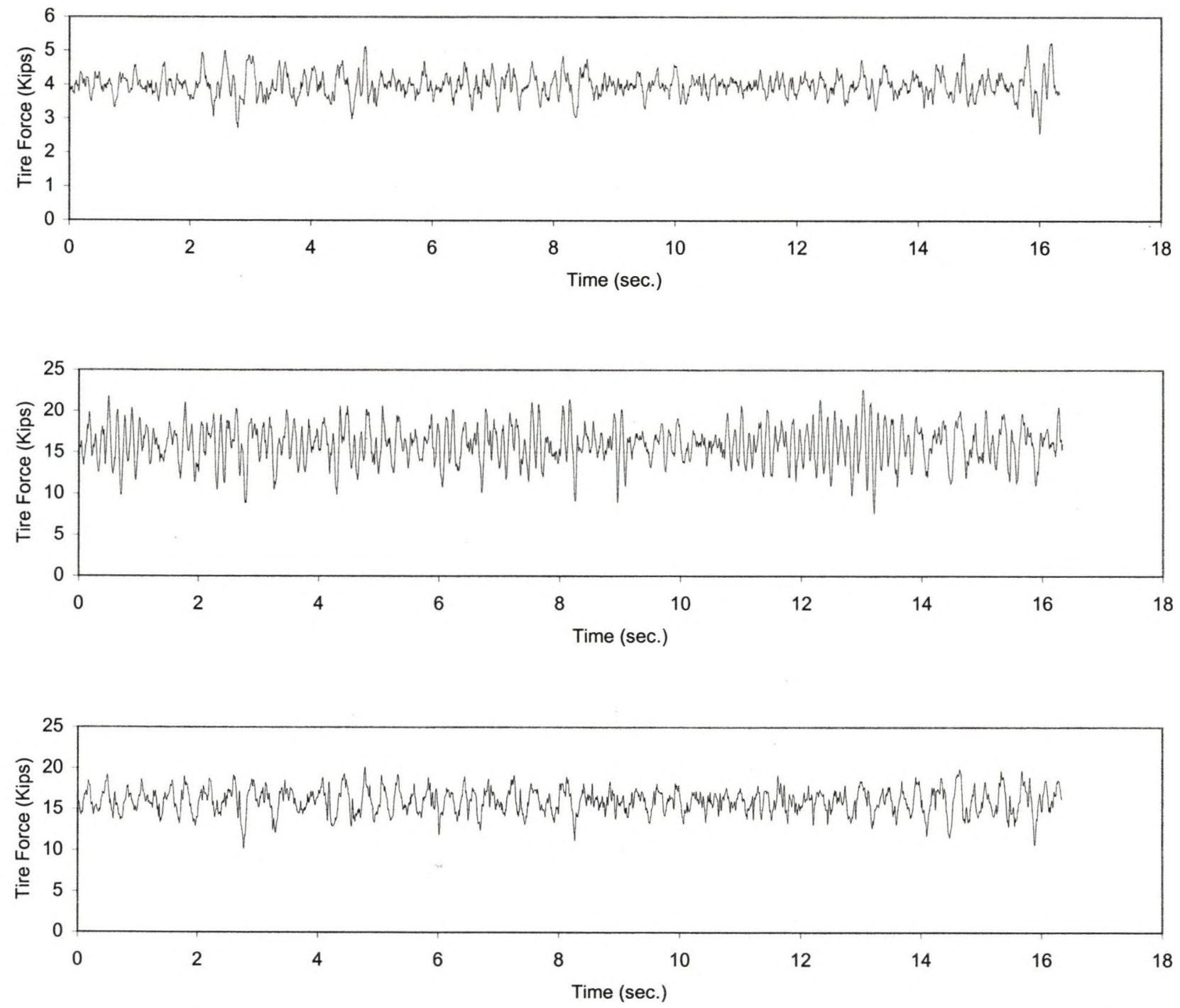

Figure A.13: Tire force history of axles one, two, and three on a very good road surface at the speed of $35 \mathrm{mph}$ 

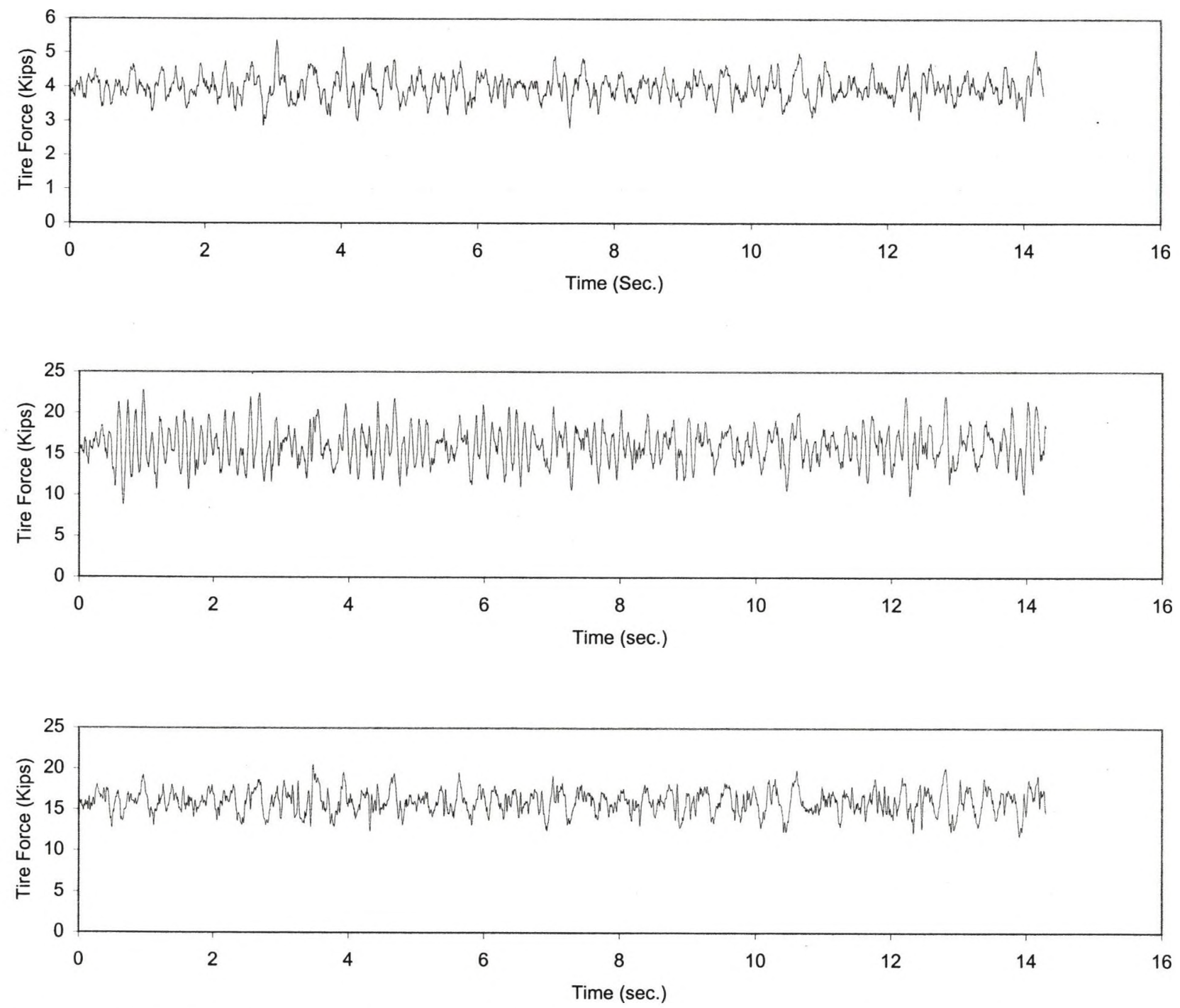

Figure A.14: Tire force history of axles one, two, and three on a very good road surface at the speed of $40 \mathrm{mph}$ 

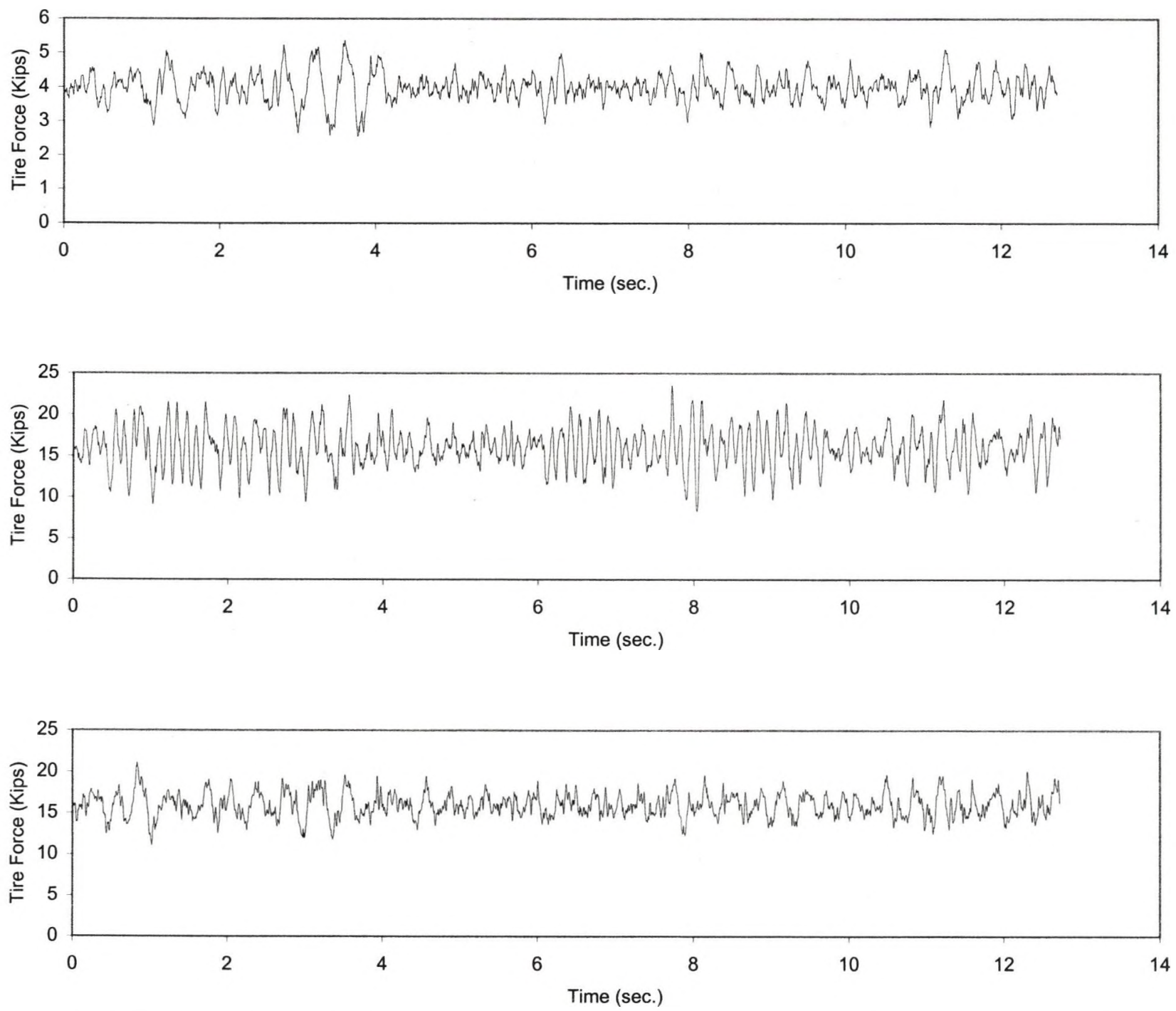

Figure A.15: Tire force history of axles one, two, and three on a very good road surface at the speed of $45 \mathrm{mph}$ 

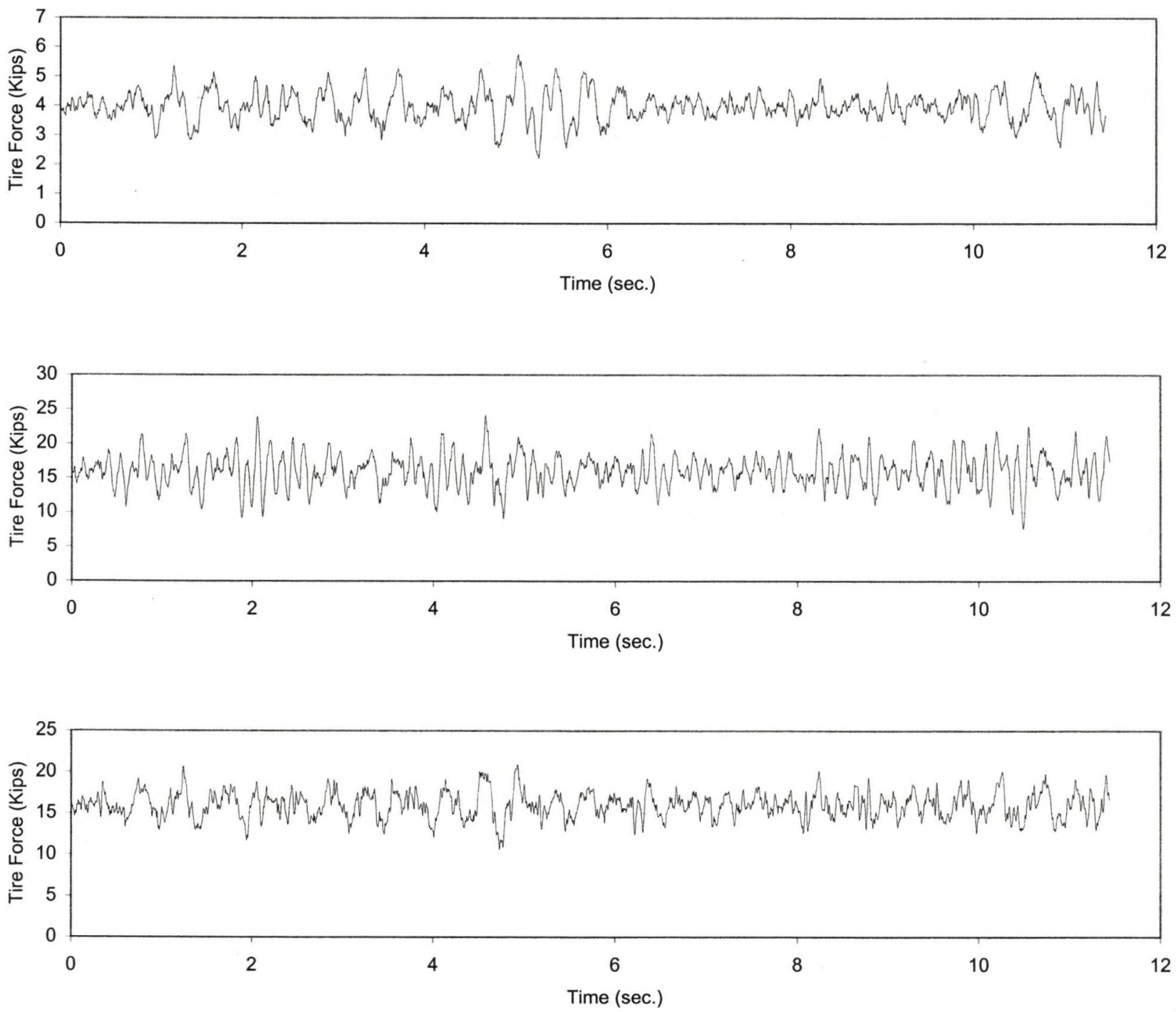

Figure A.16: Tire force history of axles one, two, and three on a very good road surface at the speed of $50 \mathrm{mph}$ 

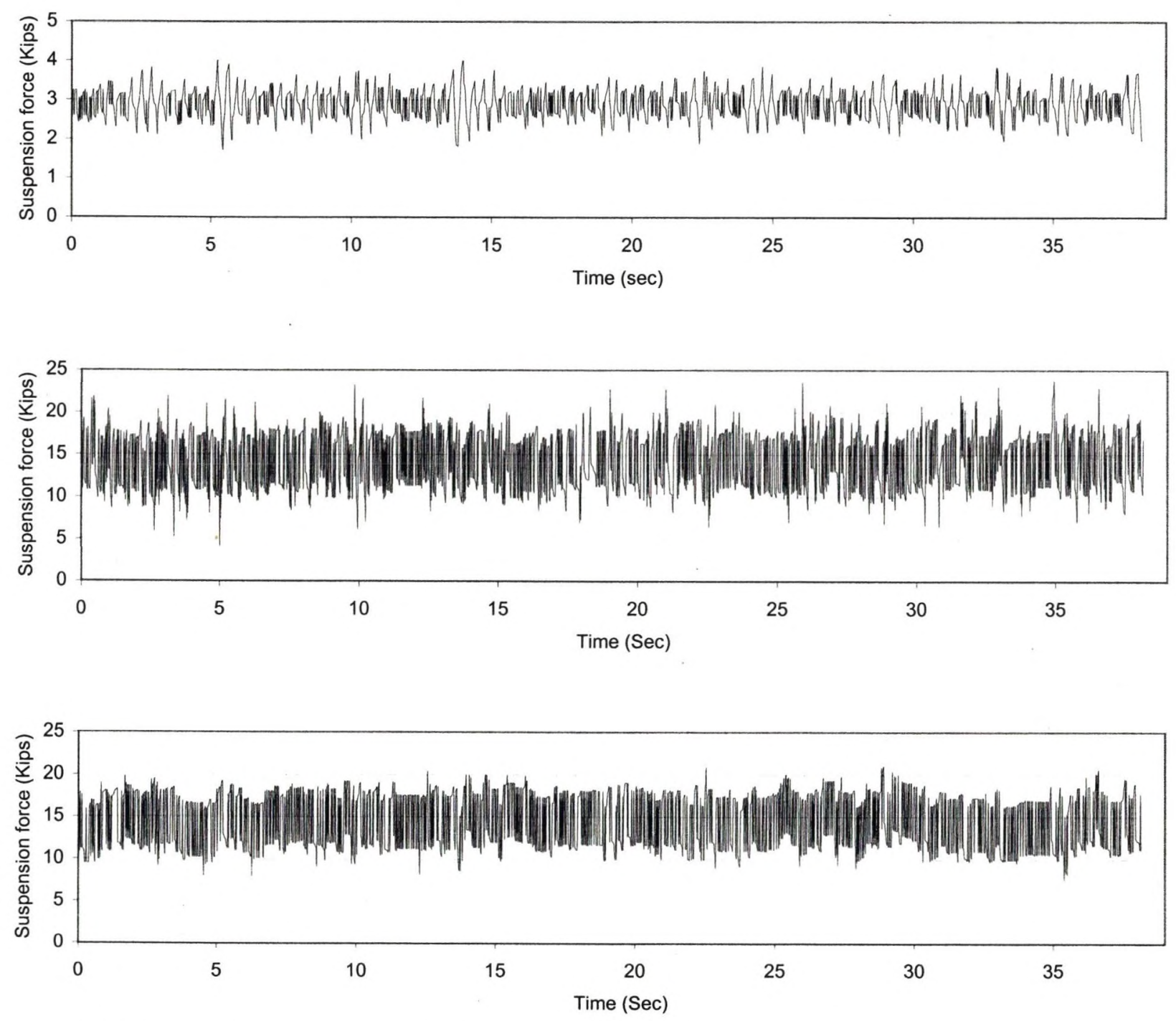

Figure A.17: Suspension force history of axles one, two, and three on a good road surface at the speed of $15 \mathrm{mph}$ 

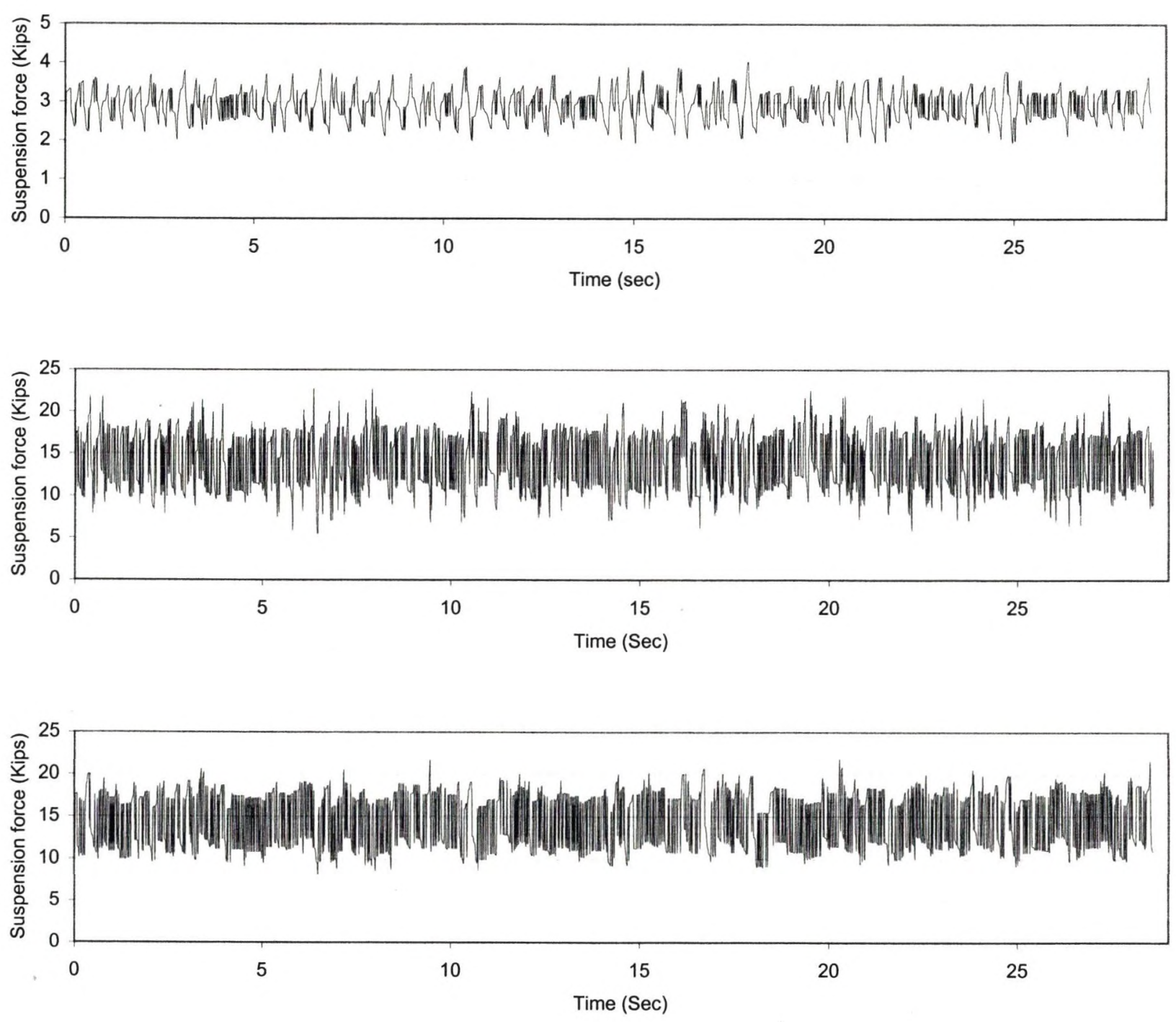

Figure A.18: Suspension force history of axles one, two, and three on a good road surface at the speed of $20 \mathrm{mph}$ 

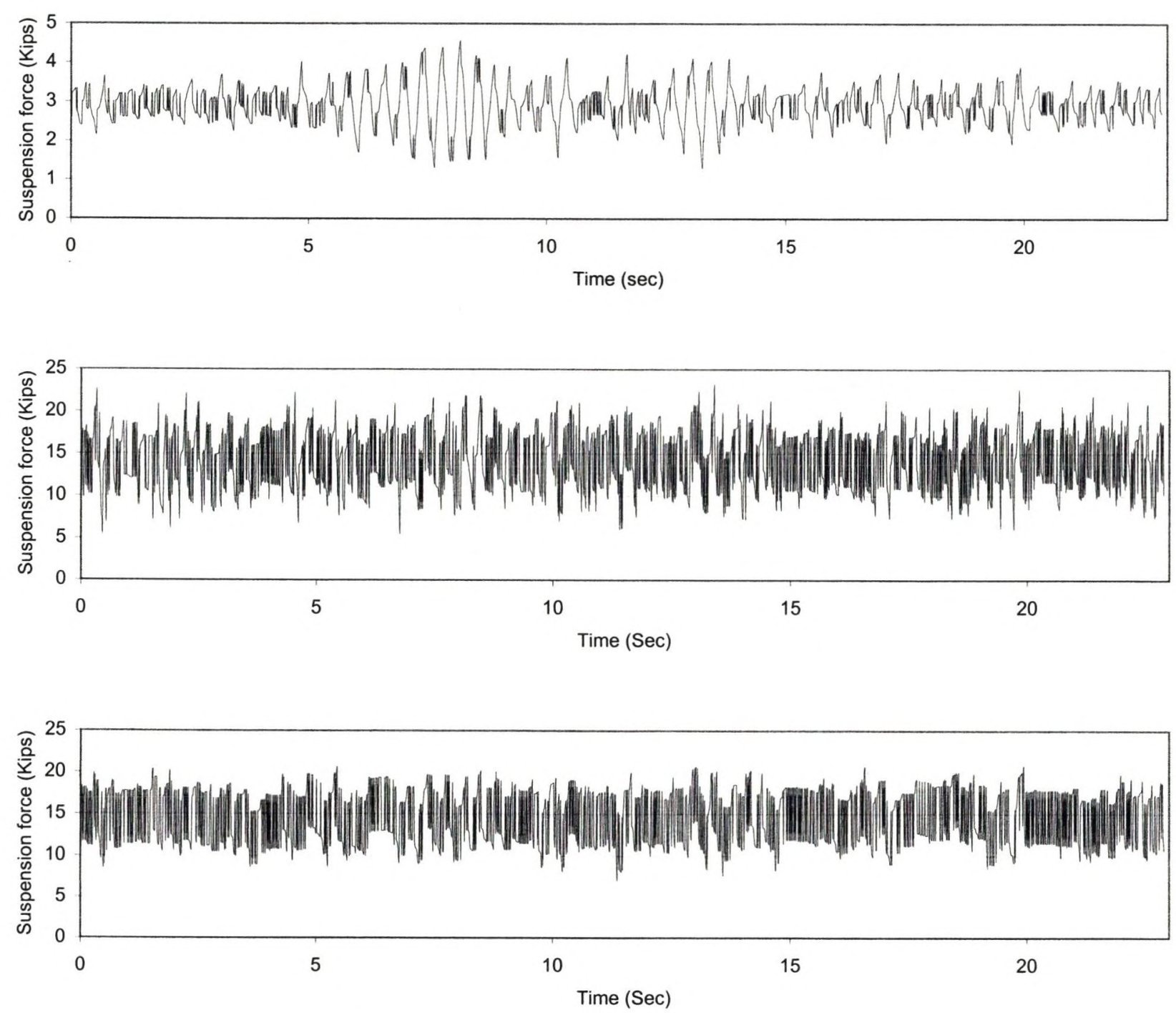

Figure A.19: Suspension force history of axles one, two, and three on a good road surface at the speed of $25 \mathrm{mph}$ 

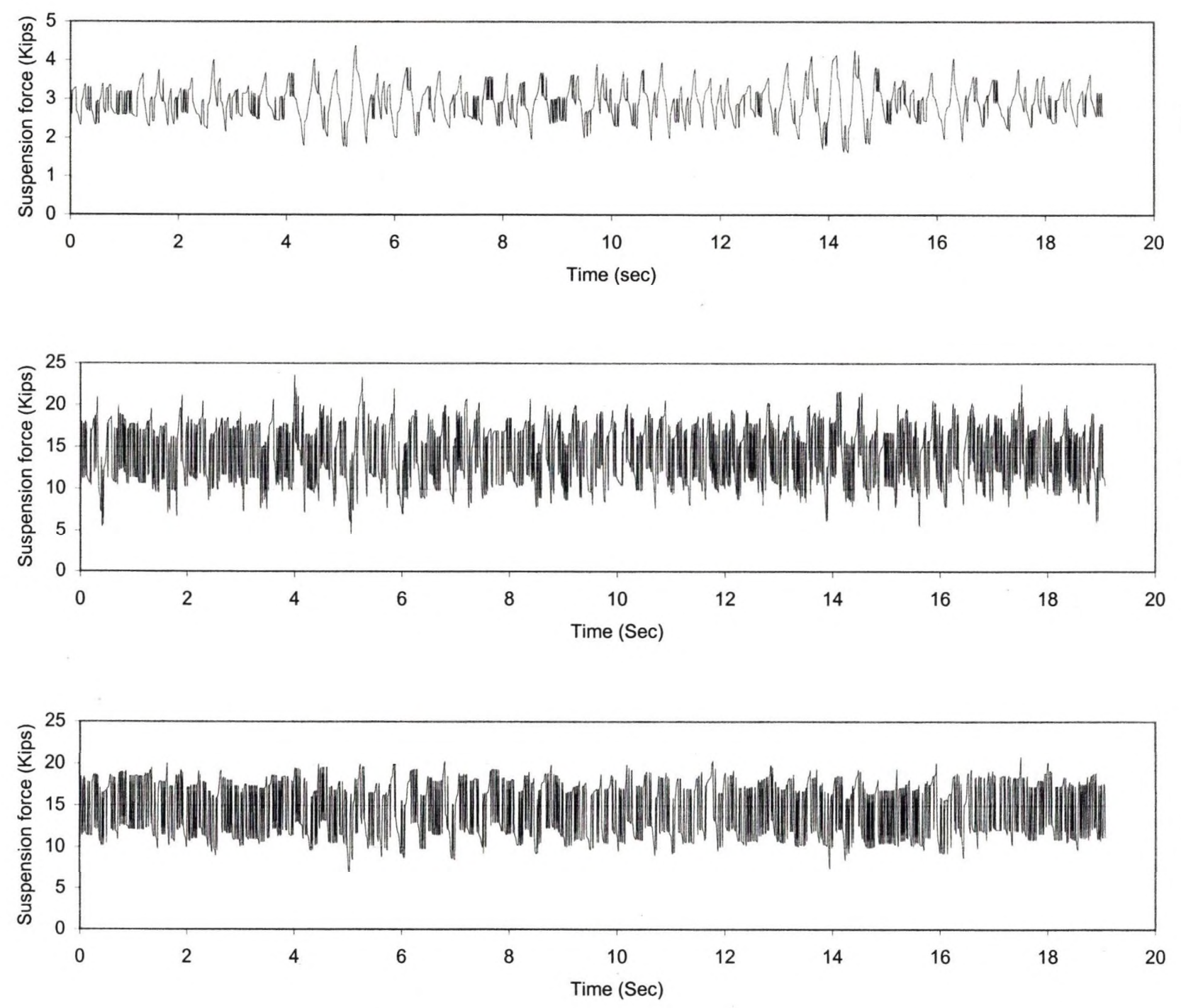

Figure A.20: Suspension force history of axles one, two, and three on a good road surface at the speed of $30 \mathrm{mph}$ 

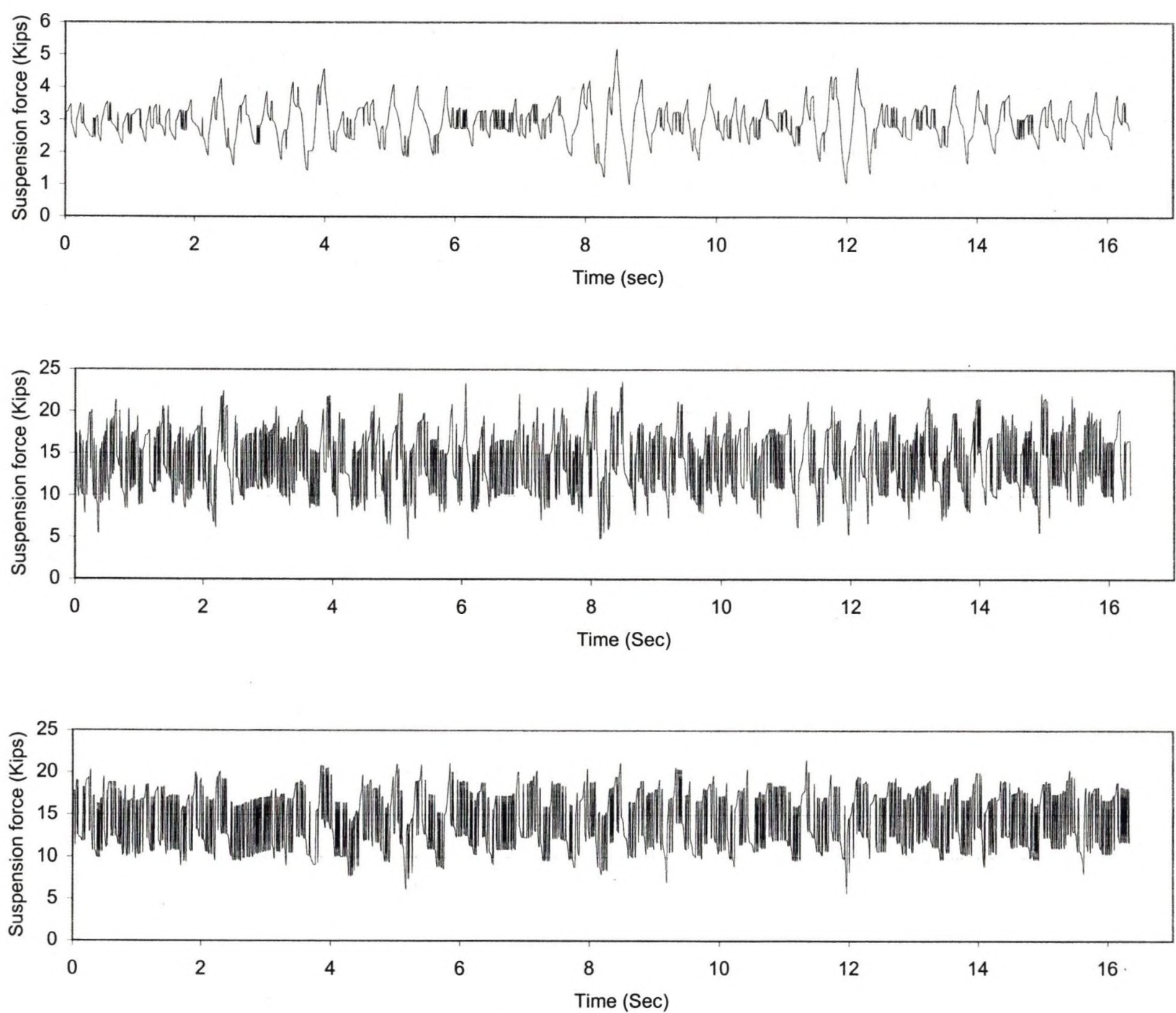

Figure A.21: Suspension force history of axles one, two, and three on a good road surface at the speed of $35 \mathrm{mph}$ 

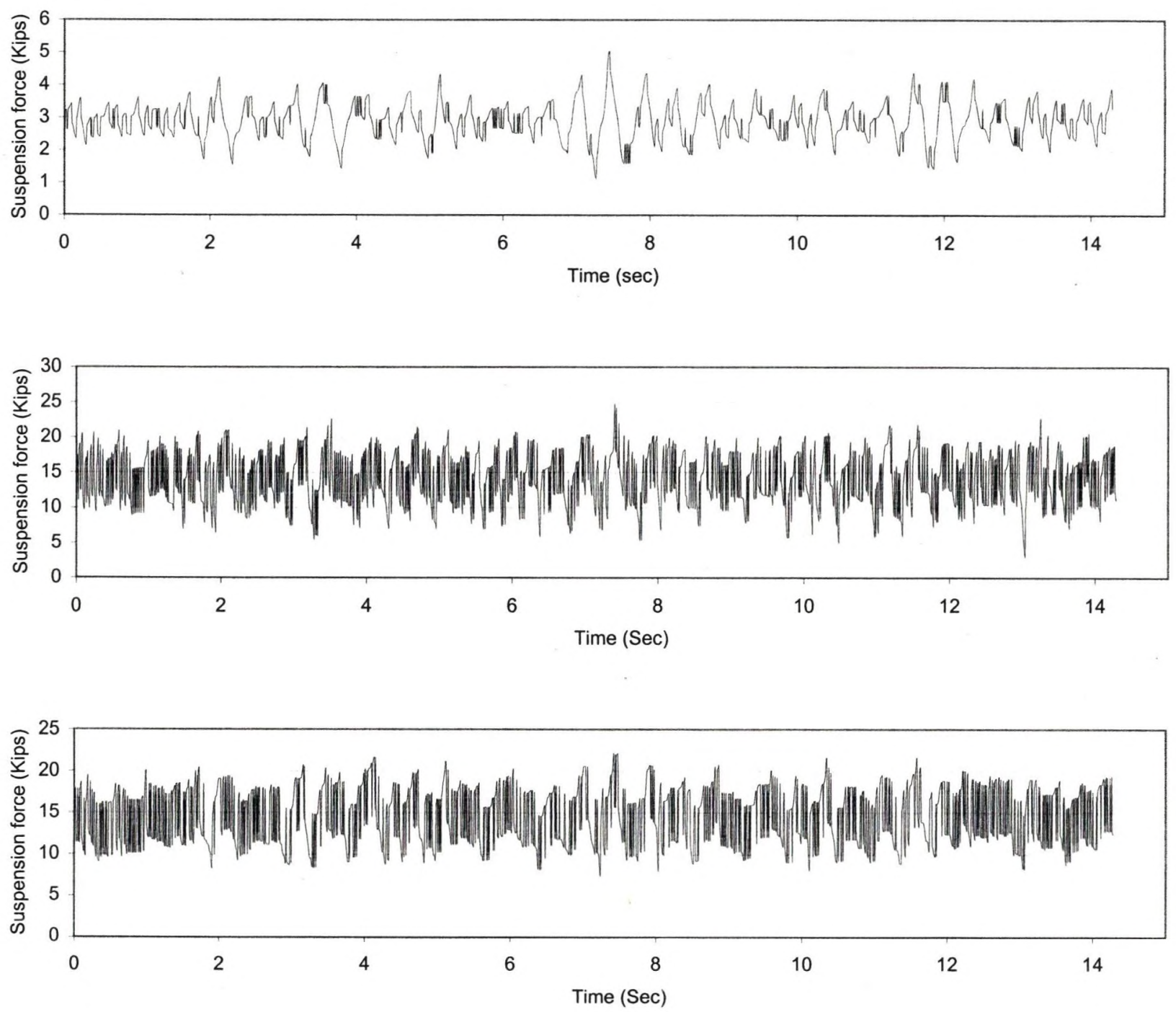

FSY5

Figure A.22: Suspension force history of axles one, two, and three on a good road surface at the speed of $40 \mathrm{mph}$ 

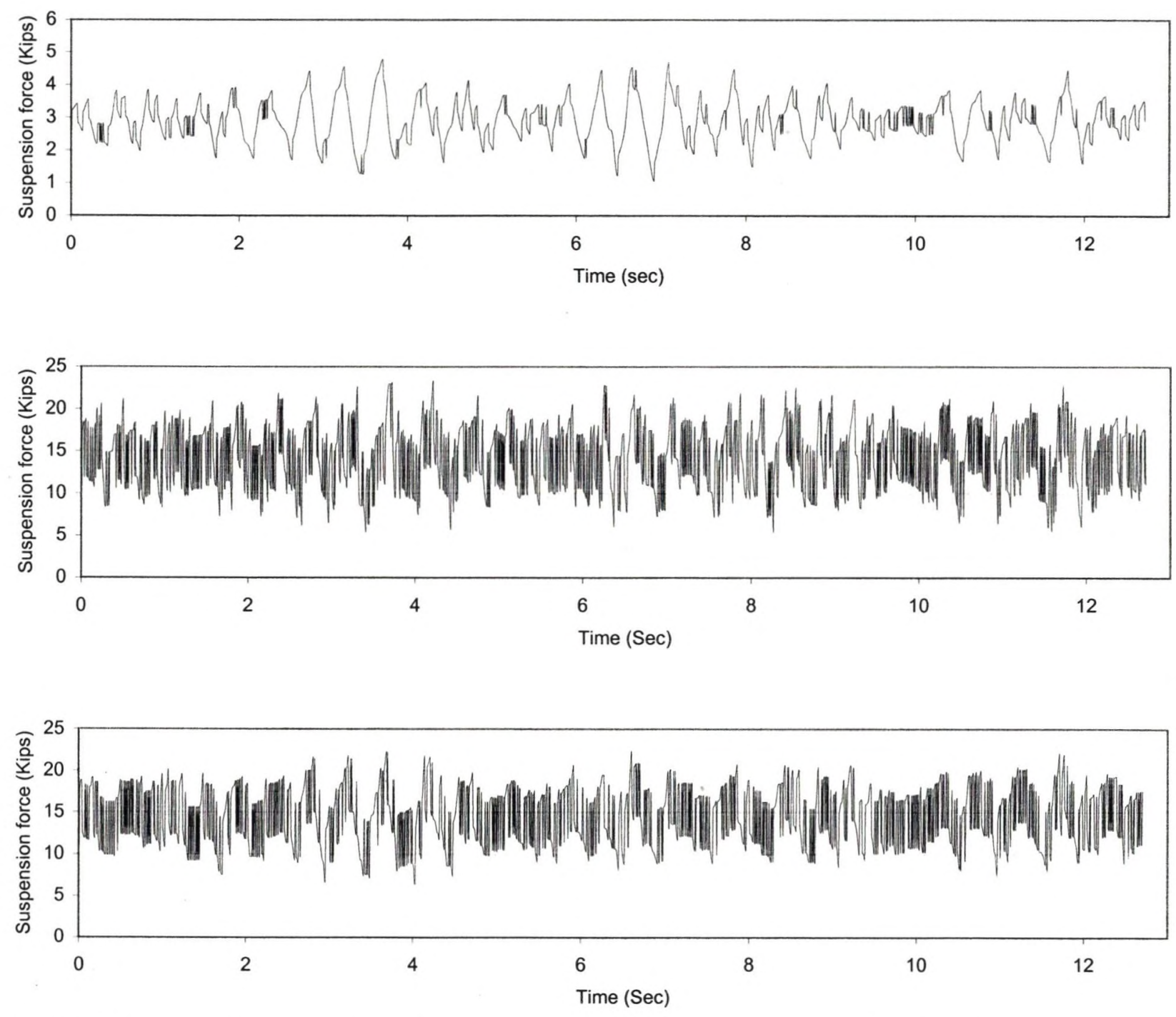

Figure A.23: Suspension force history of axles one, two, and three on a good road surface at the speed of $45 \mathrm{mph}$ 

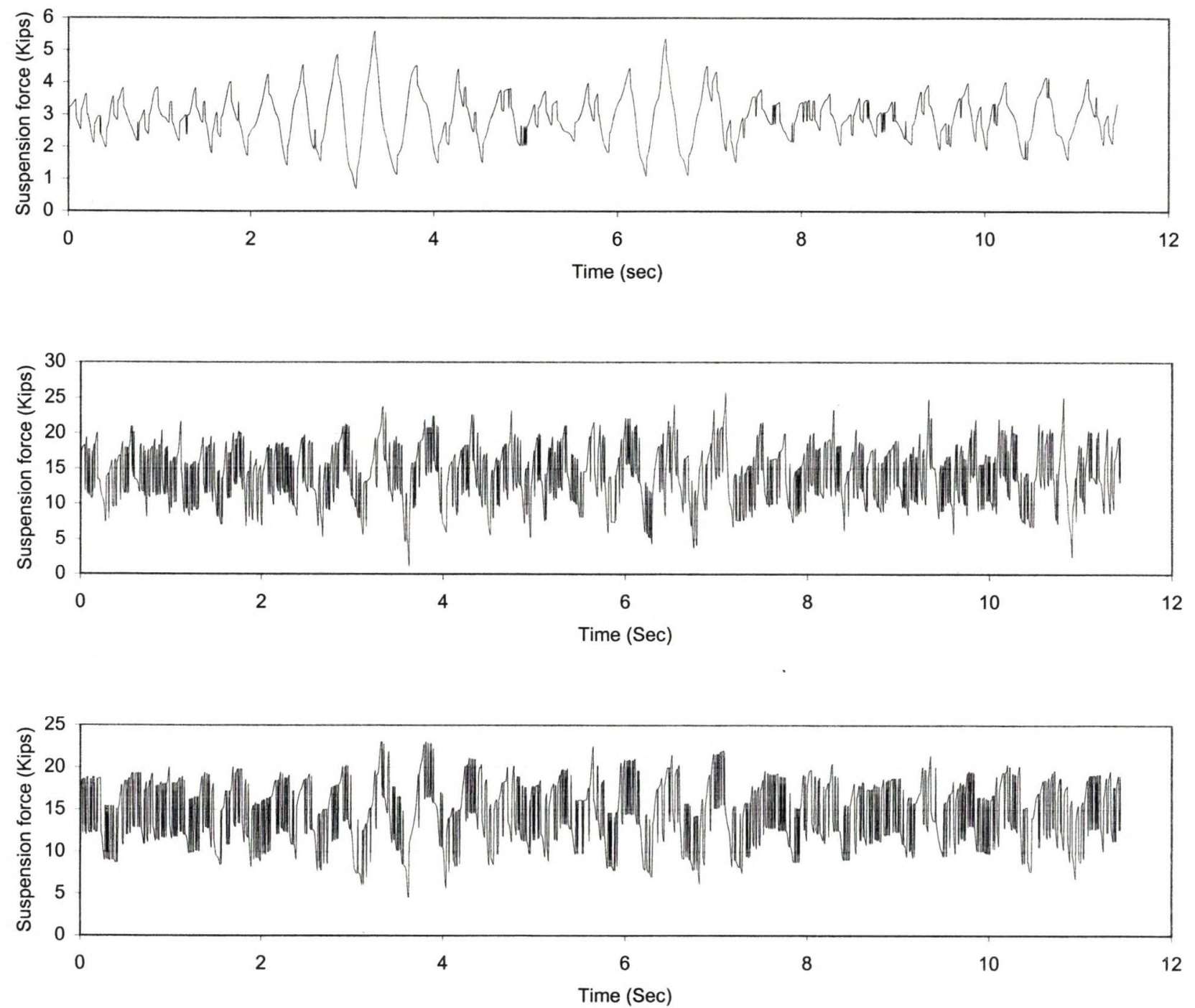

Figure A.24: Suspension force history of axles one, two, and three on a good road surface at the speed of $50 \mathrm{mph}$ 

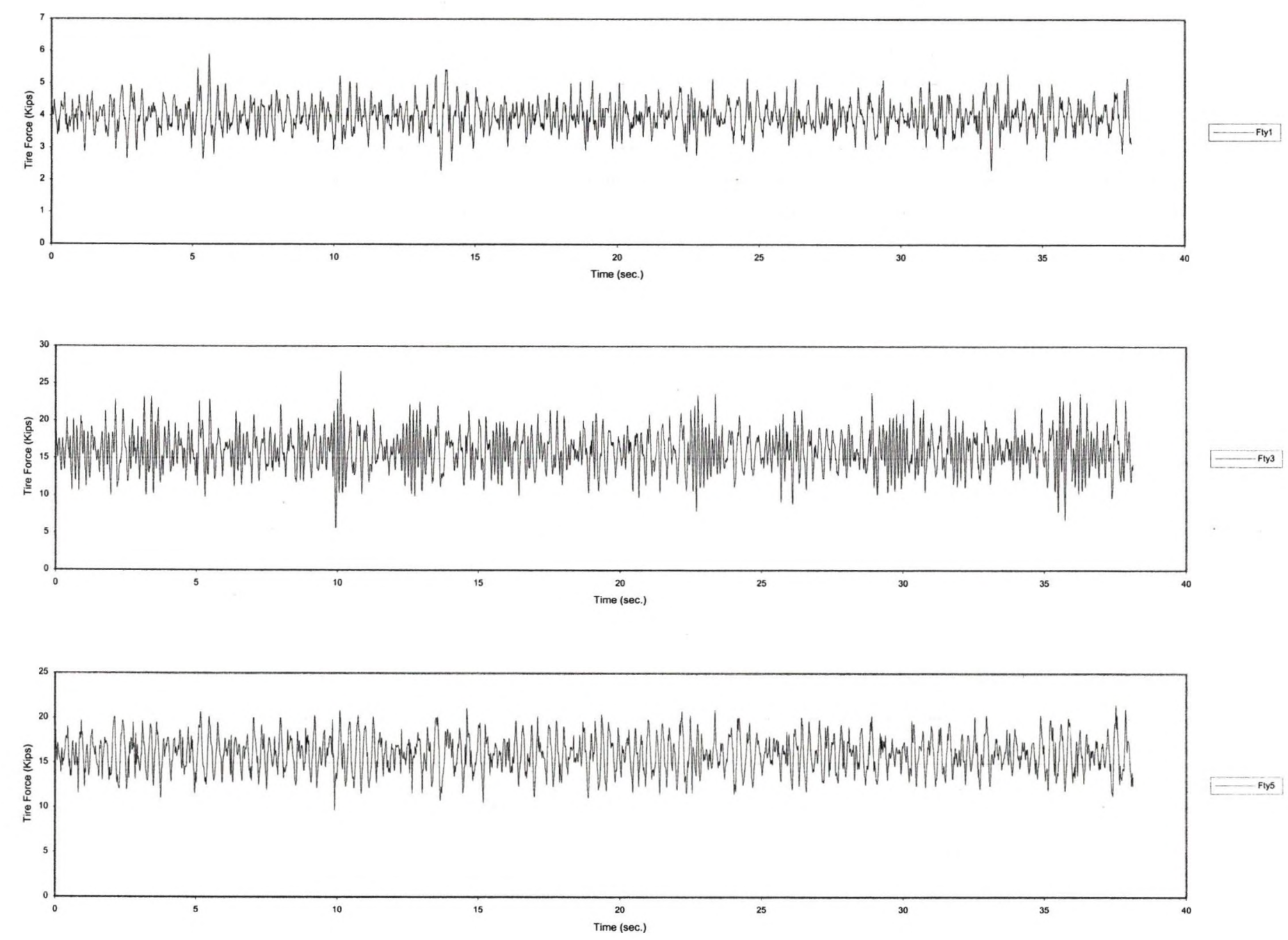

Figure A.25: Tire force history of axles one, two, and three on a good road surface at the speed of $15 \mathrm{mph}$ 

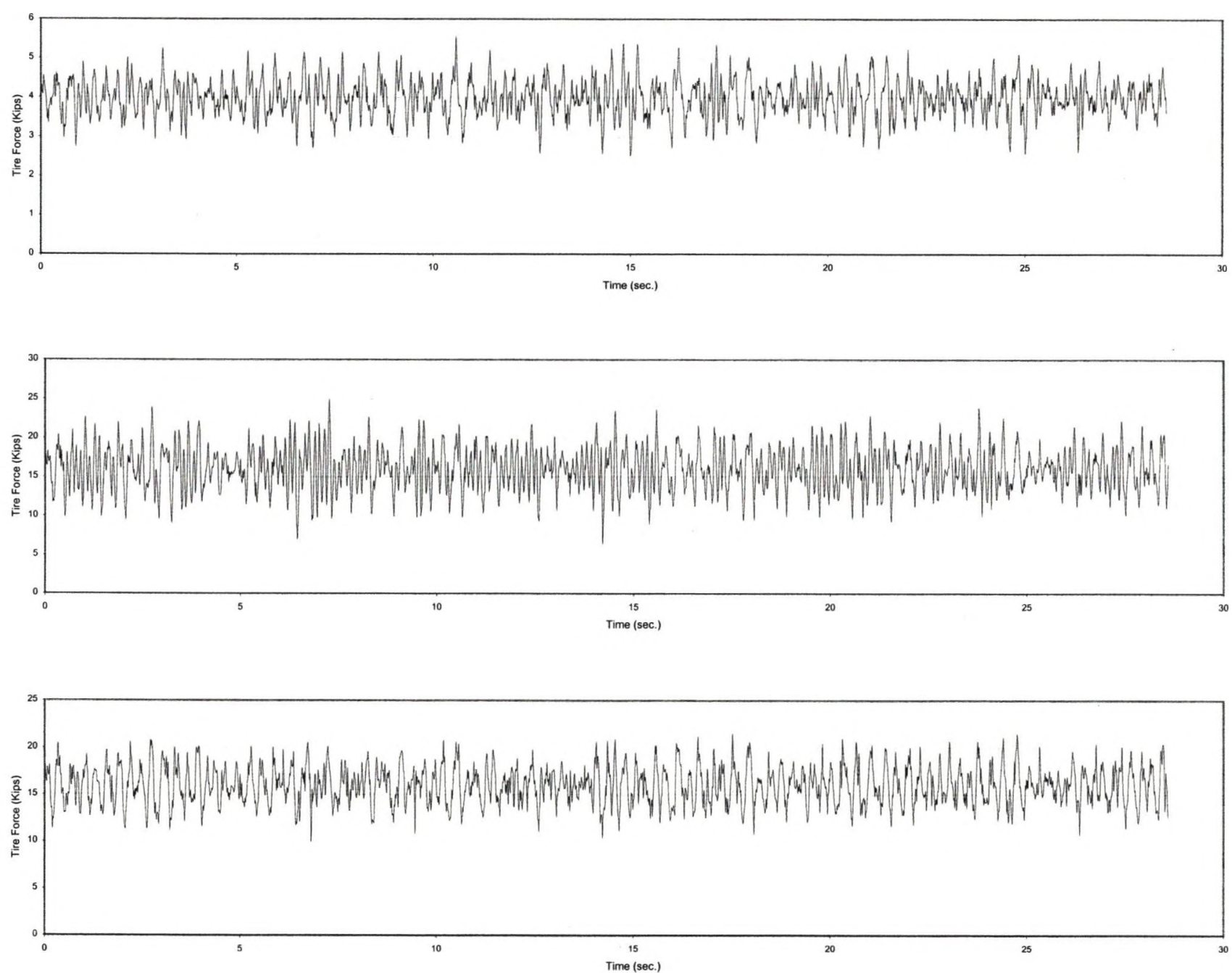

Figure A.26: Tire force history of axles one, two, and three on a good road surface at the speed of $20 \mathrm{mph}$ 

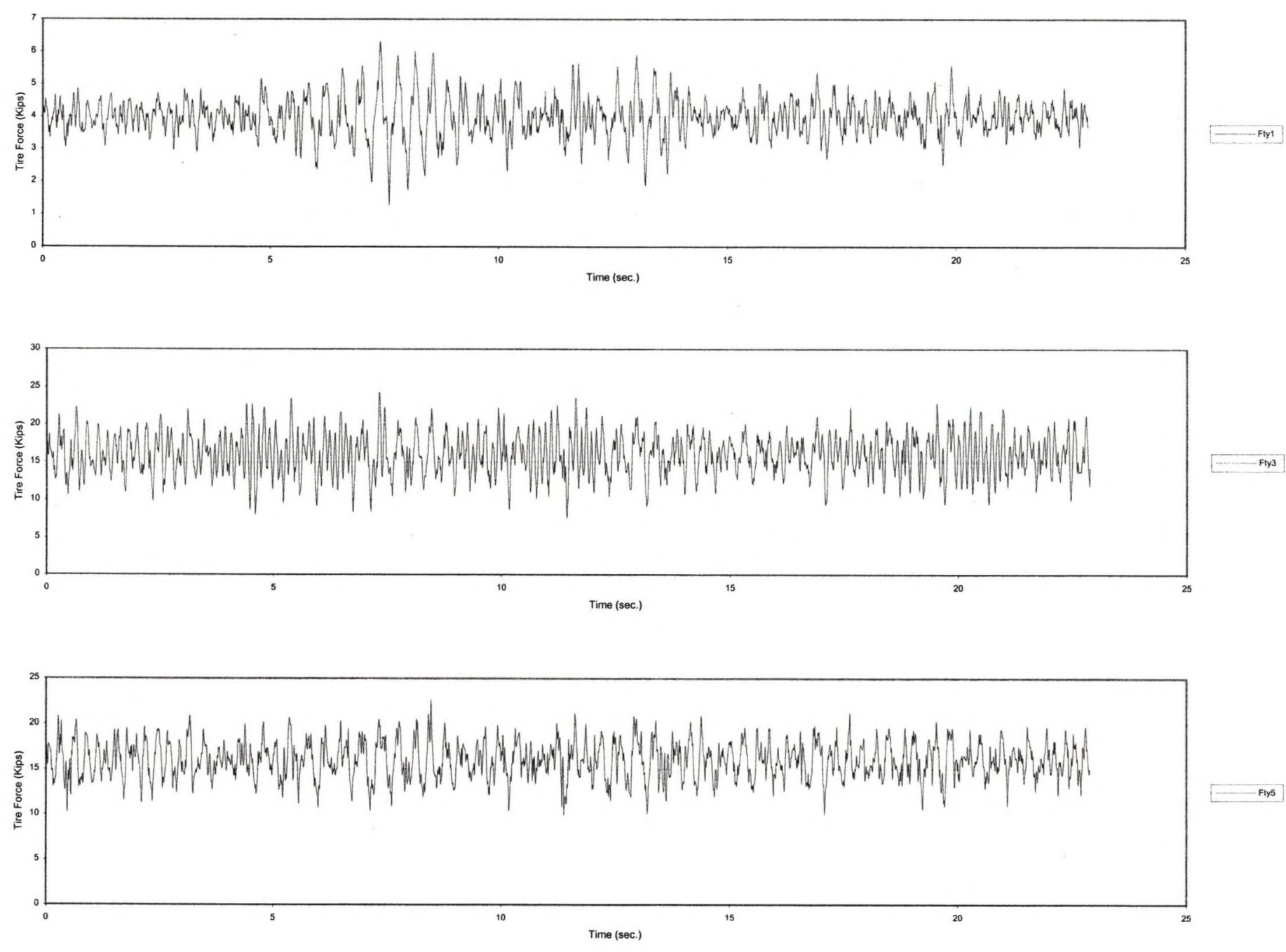

Figure A.27: Tire force history of axles one, two, and three on a good road surface at the speed of $25 \mathrm{mph}$ 

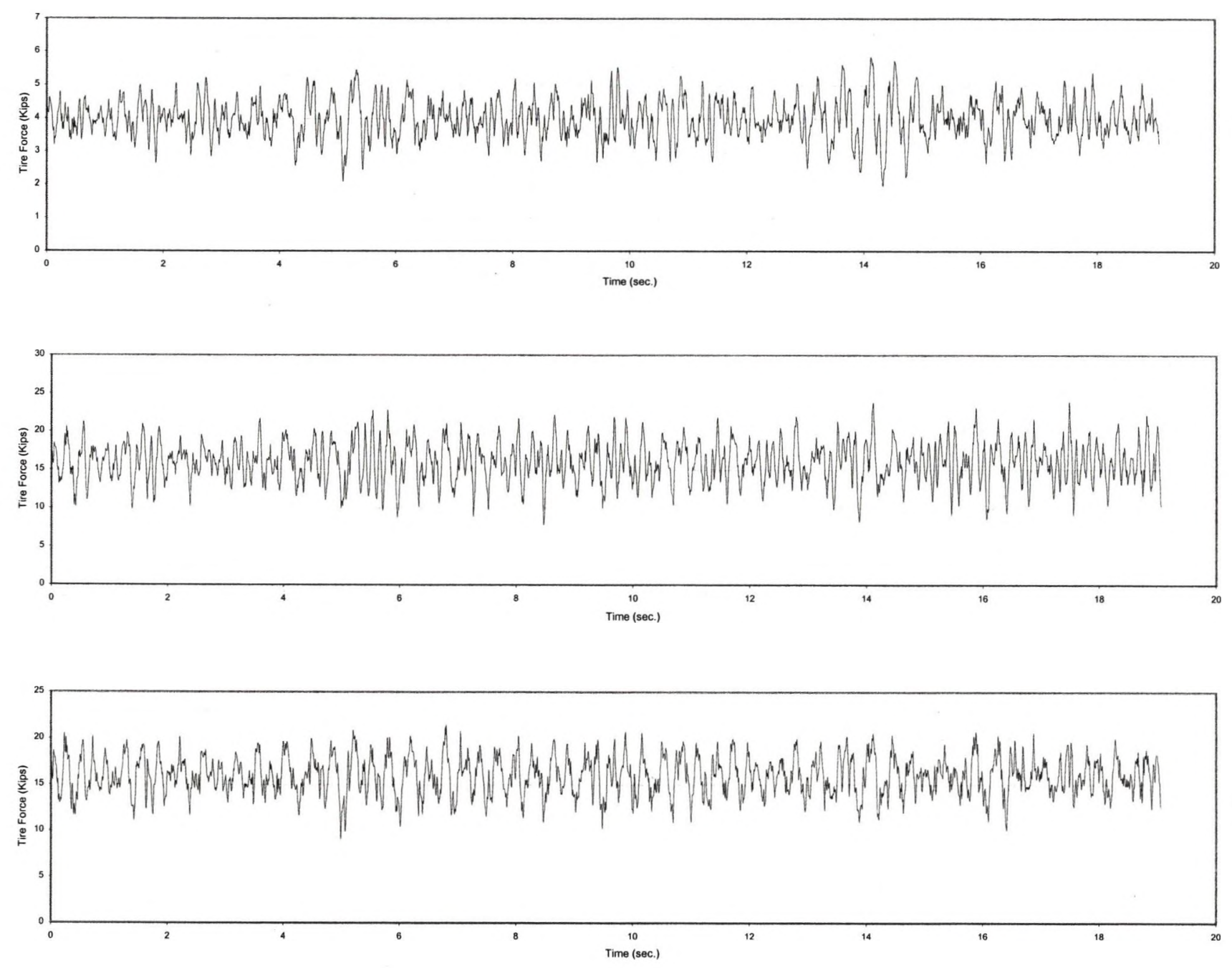

Figure A.28: Tire force history of axles one, two, and three on a good road surface at the speed of $30 \mathrm{mph}$ 

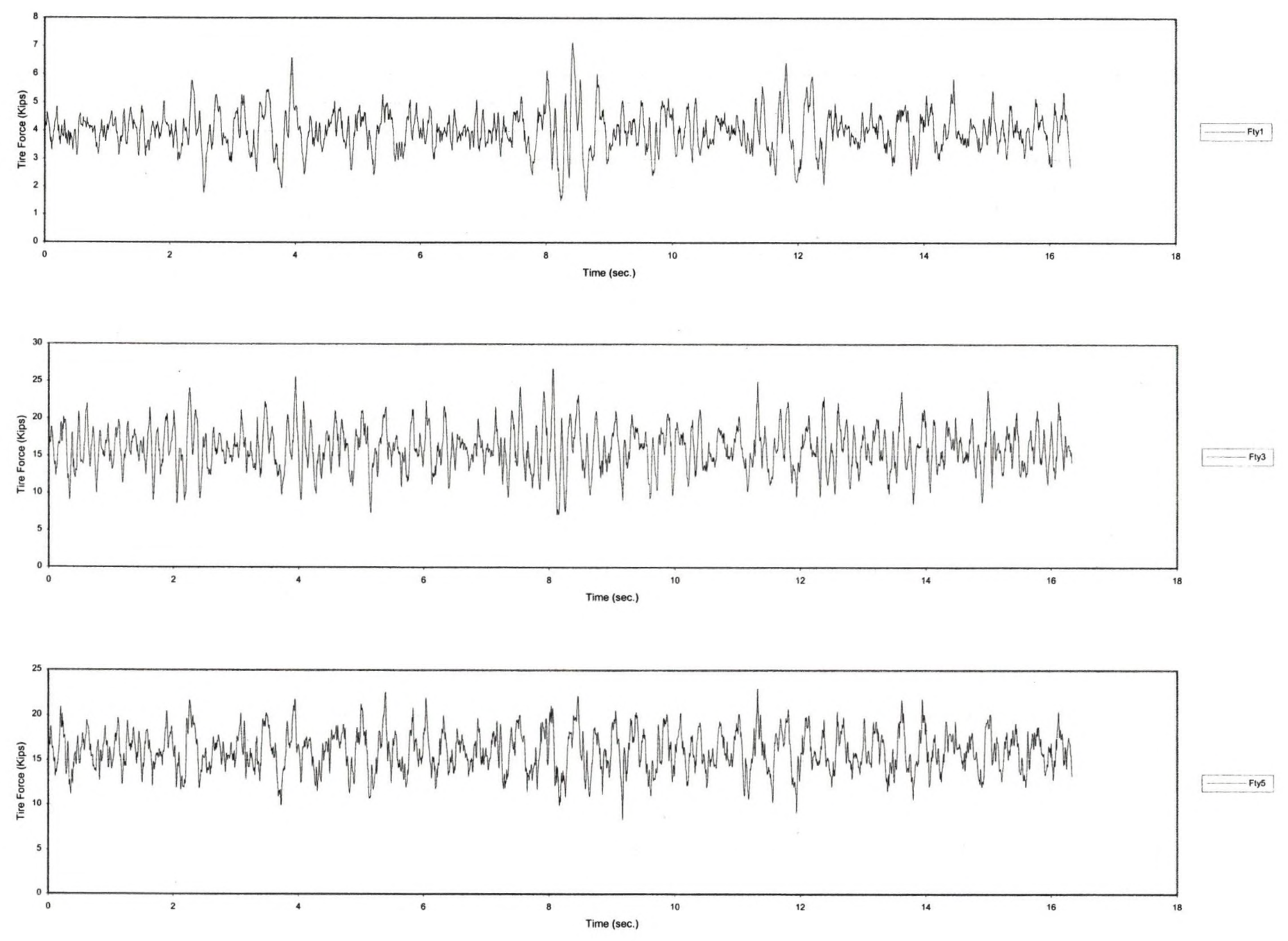

Figure A.29: Tire force history of axles one, two, and three on a good road surface at the speed of $35 \mathrm{mph}$ 

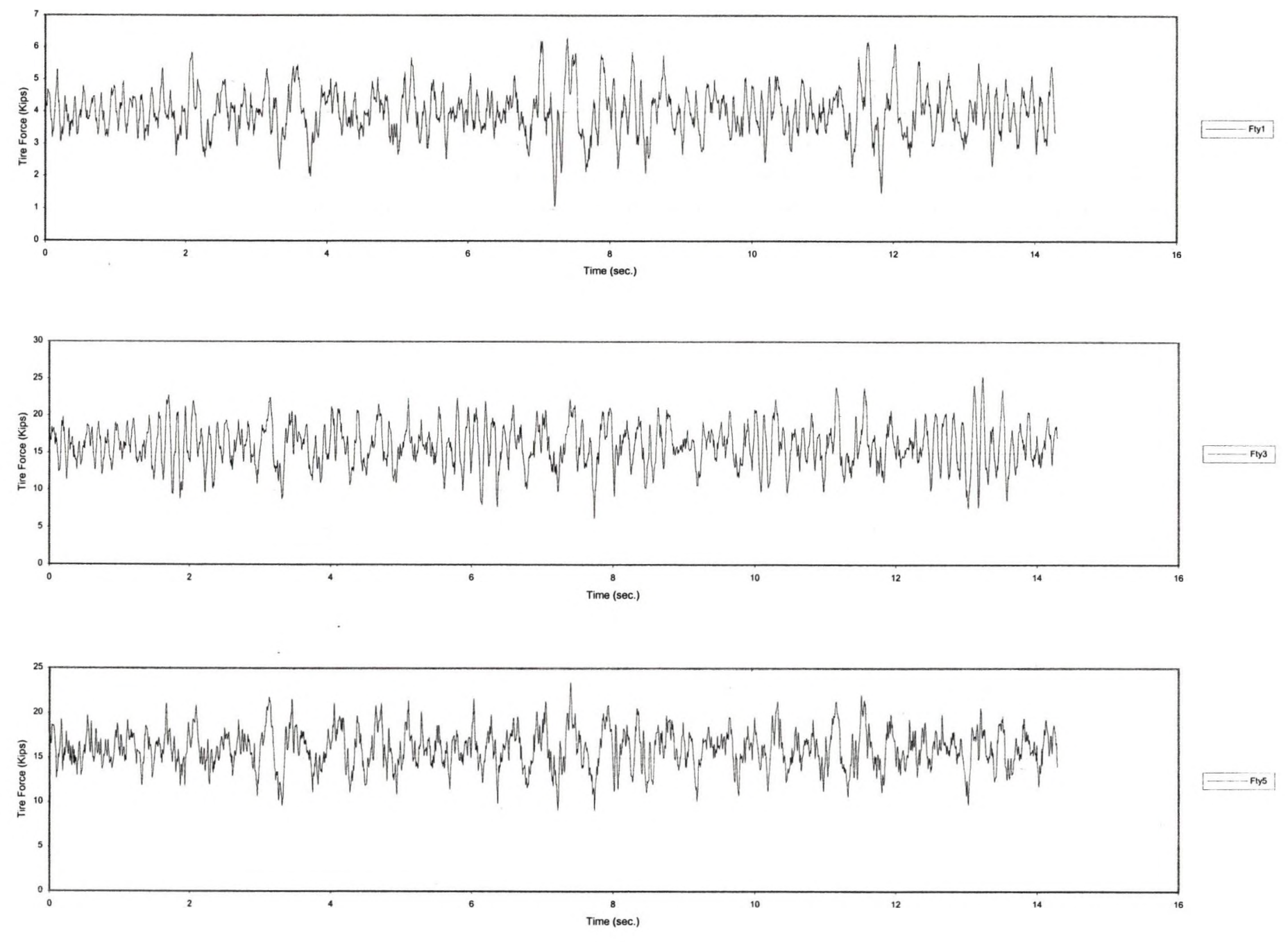

Figure A.30: Tire force history of axles one, two, and three on a good road surface at the speed of $40 \mathrm{mph}$ 

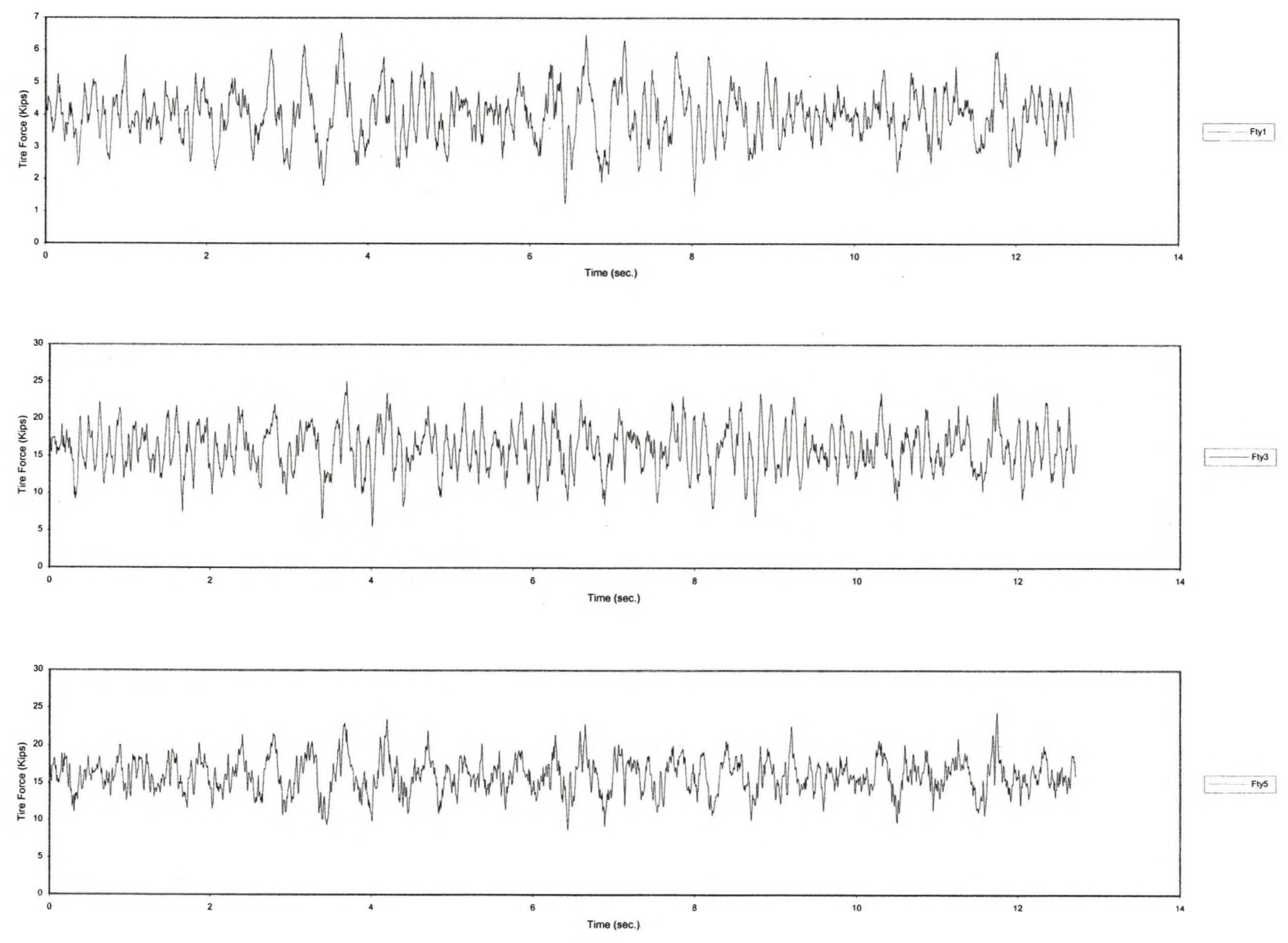

Figure A.31: Tire force history of axles one, two, and three on a good road surface at the speed of $45 \mathrm{mph}$ 

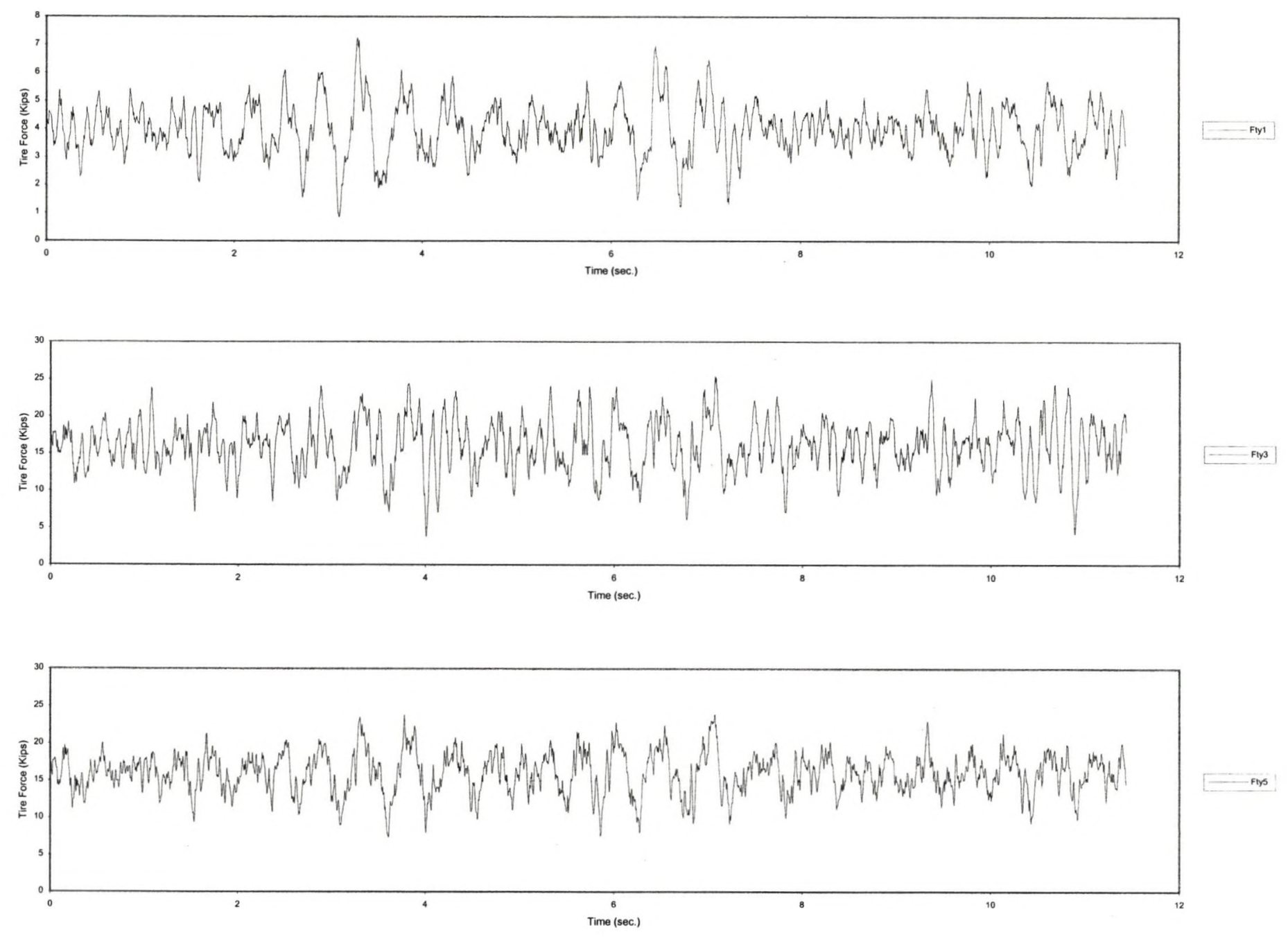

Figure A.32: Tire force history of axles one, two, and three on a good road surface at the speed of $50 \mathrm{mph}$ 
Data used in the simulation of the HS20-44 truck:

Dimensions:

Distance between the steer and tractor axles (L1)

168.000 in.

Distance between the tractor and trailer axles (L2)

168.000 in.

Distance between the steer axle and the center of the tractor (L3) $\quad \mathbf{6 6 . 8 6 4}$ in.

Distance between the tractor axle and the center of the tractor (L4) 101.136 in.

Distance between the pivot and the center of the tractor (L5) $\quad \mathbf{8 7 . 1 9 2}$ in.

Distance between the pivot and the trailer axle (L6) $\quad \mathbf{1 8 1 . 9 4 4}$ in.

Distance between the pivot and the center of the trailer (L7) $\quad \mathbf{9 2 . 0 6 4}$ in.

Distance between the trailer axle and the center of the trailer (L8) $\mathbf{8 9 . 8 8 0}$ in.

Spacing of suspensions in the steer axle (S1) $\quad \mathbf{4 4 . 0 0 0}$ in.

Spacing of suspensions in the tractor axle (S2) $\quad \mathbf{3 6 . 0 0 0}$ in.

Spacing of suspensions in the trailer axle (S3) $\quad \mathbf{3 6 . 0 0 0}$ in.

Spacing of wheels in the steer axle (D1) $\quad \mathbf{6 8 . 0 0 0}$ in.

Spacing of wheels in the tractor axle (D2) $\quad \mathbf{7 2 . 0 0 0}$ in.

Spacing of wheels in the trailer axle (D3) $\quad \mathbf{7 2 . 0 0 0}$ in.

Stiffness and damping characteristics:

Stiffness of suspension in the steer axle (Ksy1) $\quad 1.3850 \quad \mathrm{kips} / \mathrm{in}$.

Stiffness of suspension in the steer axle (Ksy2) $\quad 1.3850 \quad$ kips/in.

Stiffness of suspension in the tractor axle (Ksy3) $\quad \mathbf{1 0 . 8 6 5 0}$ kips/in.

Stiffness of suspension in the tractor axle (Ksy4) $\quad \mathbf{1 0 . 8 6 5 0} \quad$ kips/in.

Stiffness of suspension in the trailer axle (Ksy5) $\quad \mathbf{1 1 . 2 4 1 0} \mathrm{kips} / \mathrm{in}$. 
Stiffness of suspension in the trailer axle (Ksy6)

Stiffness of tire in the steer axle (Kty1)

Stiffness of tire in the steer axle (Kty2)

Stiffness of tire in the tractor axle (Kty3)

Stiffness of tire in the tractor axle (Kty4)

Stiffness of tire in the trailer axle (Kty5)

Stiffness of tire in the trailer axle (Kty6)

Damping coefficient in the steer axle (Dsy1)

Damping coefficient in the steer axle (Dsy2)

Damping coefficient in the tractor axle (Dsy3)

Damping coefficient in the tractor axle (Dsy4)

Damping coefficient in the trailer axle (Dsy5)

Damping coefficient in the trailer axle (Dsy6)

Damping coefficient in tire (Dty1)

Damping coefficient in tire (Dty2)

Damping coefficient in tire (Dty3)

Damping coefficient in tire (Dty4)

Damping coefficient in tire (Dty5)

Damping coefficient in tire (Dty6)

Friction Force in the steer axle (Fy1)

Friction Force in the steer axle (Fy2)

Friction Force in the tractor (Fy3)

Friction Force in the tractor (Fy4)
11.2410 kips/in.

4.9960 kips/in.

4.9960 kips/in.

$20.0000 \mathrm{kips} / \mathrm{in}$.

$20.0000 \mathrm{kips} / \mathrm{in}$.

20.0240 kips/in.

$20.0240 \mathrm{kips} / \mathrm{in}$.

0.0125 kips-sec/in.

0.0125 kips-sec/in.

0.0425 kips-sec/in.

0.0425 kips-sec/in.

$\mathbf{0 . 0 4 1 0}$ kips-sec/in.

0.0410 kips-sec/in.

0.0000 kips-sec/in.

0.0000 kips-sec/in.

0.0000 kips-sec/in.

0.0000 kips-sec/in.

0.0000 kips-sec/in.

0.0000 kips-sec/in.

0.3000 kips.

0.3000 kips.

3.2000 kips.

3.2000 kips. 
Friction Force in the trailer (Fy5)

Friction Force in the trailer (Fy6)
3.2000 kips.

3.2000 kips.

Mass and Mass Moment of Inertias:

Mass of the tractor (mt1)

Roll mass moment of inertia of the tractor (Ixt1)

Pitch mass moment of inertia of the tractor (Izt1)

Mass of the trailer (mt2)

Roll mass moment of inertia of the trailer (Ixt2)

Pitch mass moment of inertia of the trailer (Izt2)

Mass of the steer axle (ma1)

Roll mass moment of inertia of the steer axle (Ixa1)

Mass of the tractor axle (ma2)

Roll mass moment of inertia of the tractor axle (Ixa2)

Mass of the trailer axle (ma3)

Roll mass moment of inertia of the trailer axle (Ixa3)
0.01491 kips-(sec)2 /in.

17.88804 kips-in-(sec)2.

75.60312 kips-in-(sec)2.

0.14907 kips-(sec)2 /in.

293.3665 kips-in-(sec)2.

1603.547 kips-in-(sec)2.

$\mathbf{0 . 0 0 5 5 9}$ kips-(sec)2 /in.

4.3602 kips-in-(sec)2.

0.00932 kips-(sec)2 /in.

$\mathbf{7 . 2 6 6 9 6}$ kips-in-(sec)2.

$\mathbf{0 . 0 0 7 4 5 ~ k i p s - ( s e c ) 2 / i n . ~}$

5.81364 kips-in-(sec)2. 


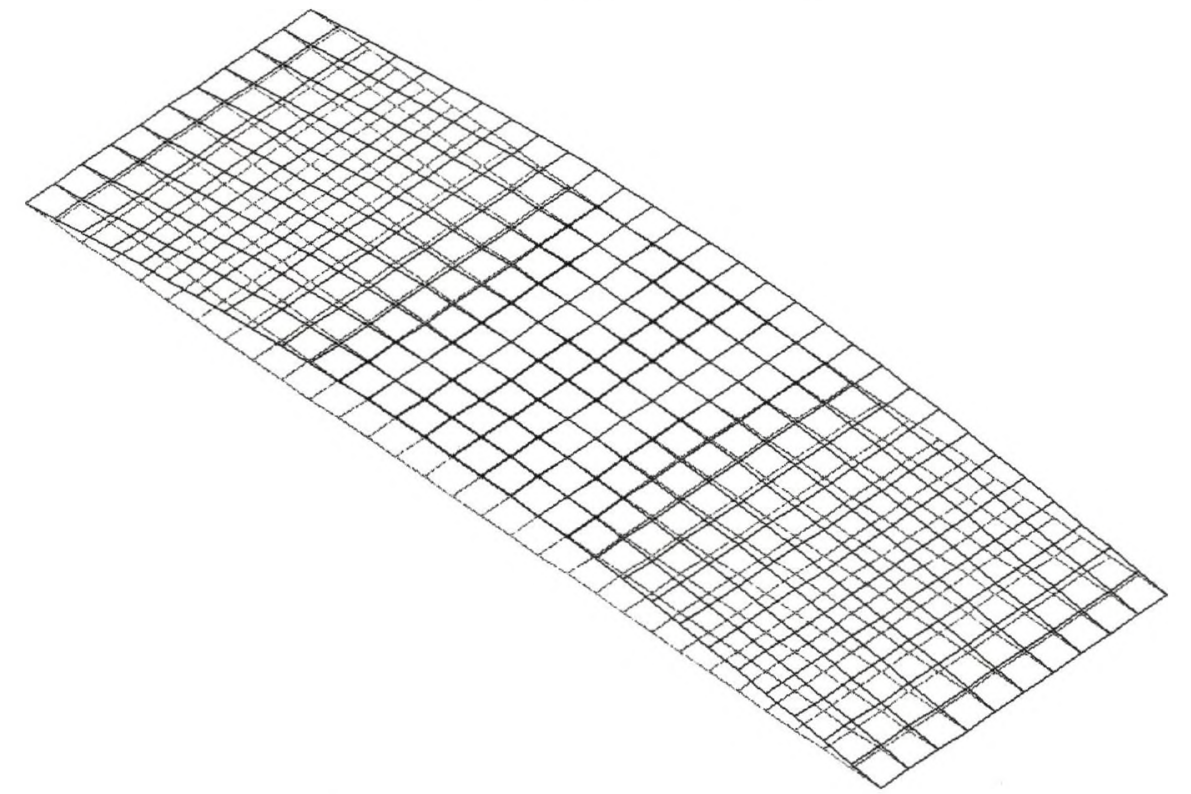

MODE SHAPE PLOT

$M X \mathrm{DEF}=2.53 \mathrm{E}-\mathrm{O} 2$

NODE NO. $=16$

SCALE $=1.0$

(MAPPED SCALING)

MODE NO. $=1$ FREQUENCY $=2.96837 \mathrm{E}+00 \mathrm{~Hz}$

Plate- $12 \mathrm{ft} \times 36 \mathrm{ft}$

EMRC-NISA/DISPLAY JUL/31/06 $12: 12: 22$

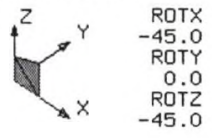

Figure B.1: First mode shape of the isotropic plate (12ft x $36 \mathrm{ft})$ in NISA

DISPLAY III - GEOMETRY MODELING SYSTEM ( 12.0 .0$)$ PRE/POST MODULE

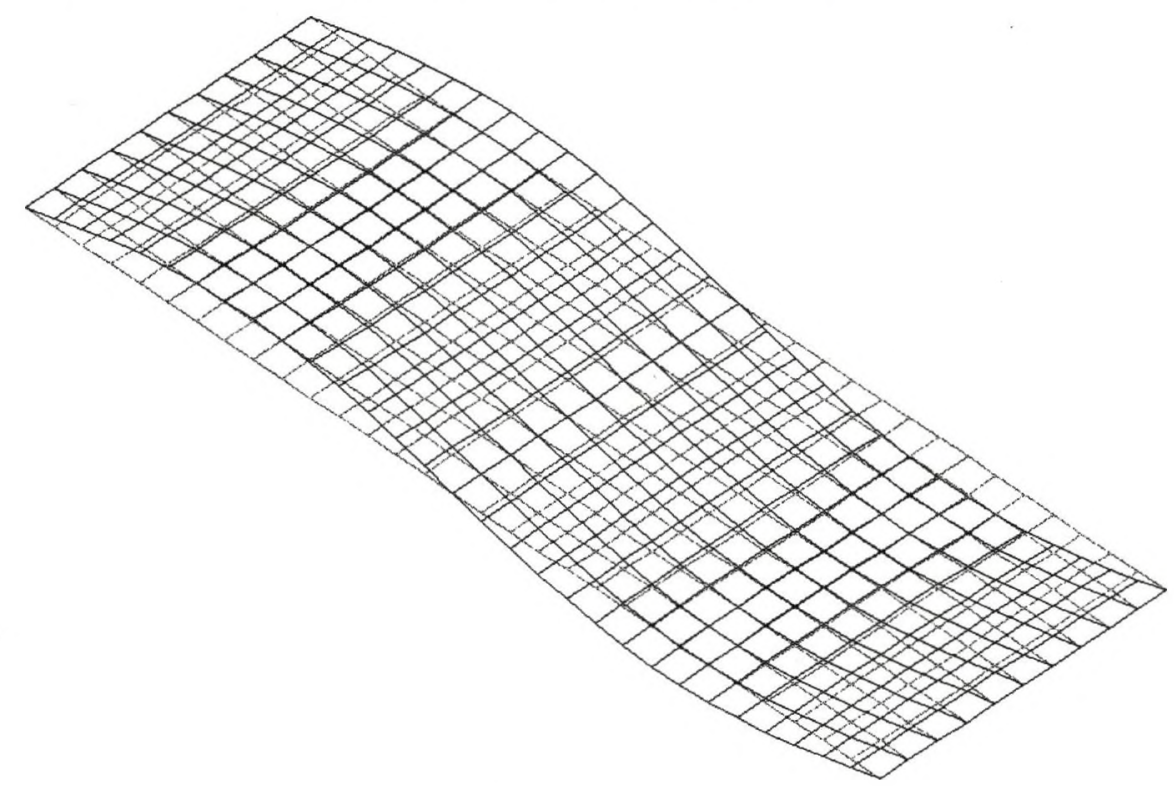

MODE NO. $=2$ FREQUENCY $=1.20205 E+01 \mathrm{~Hz}$

Plate- $12 \mathrm{ft} \times 36 \mathrm{ft}$
MODE SHAPE PLOT

$M X \mathrm{DEF}=2.63 \mathrm{E}-02$

NODE NO. $=8$

SCALE $=1.0$

(MAPPED SCALING)
EMRC-NISA/DISPLAY

JUL/31/06 12:13:04

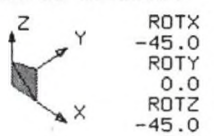

Figure B.2: Second mode shape of the isotropic plate $(12 \mathrm{ft} \times 36 \mathrm{ft})$ in NISA 
MODE SHAPE PLOT

$M X \quad D E F=4.26 E-02$

NODE NO $=16$

SCALE $=1.0$

(MAPPED SCALING)

MODE NO. $=3$ FREQUENCY $=1.23302 \mathrm{E}+01 \mathrm{~Hz}$

Plate $-12 \mathrm{ft} \times 36 \mathrm{ft}$

EMRC-NISA/DISPLAY JUL/31/06 $12: 13: 50$

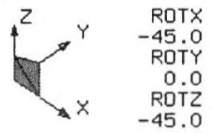

Figure B.3: Third mode shape of the isotropic plate (12ft x $36 \mathrm{ft})$ in NISA

DISPLAY III - GEOMETRY MODELING SYSTEM ( 12.0 .0 ) PRE/POST MODULE

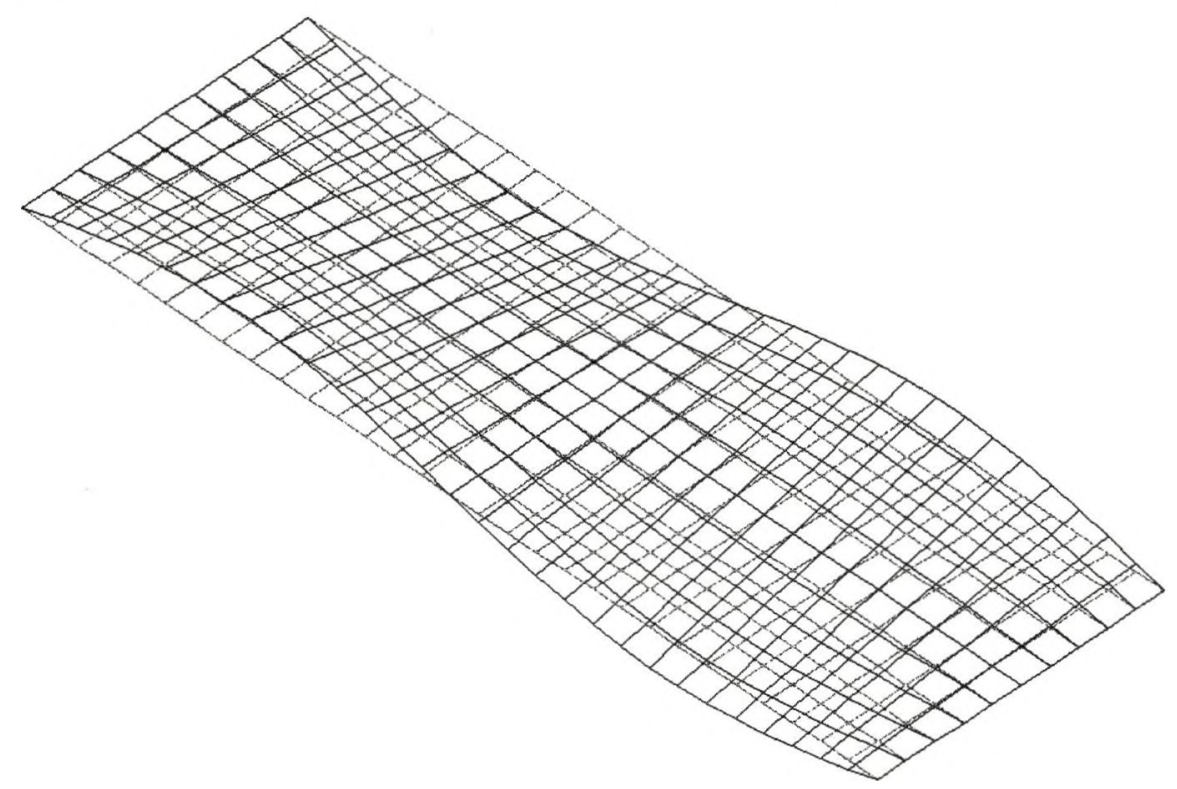

MODE SHAPE PLOT $M X$ DEF $=4.25 E-02$ NODE NO. $=8$ SCALE $=1.0$ (MAPPED SCALING)

MODE NO. $=4$ FREQUENCY $=2.68394 \mathrm{E}+01 \mathrm{~Hz}$ Plate- $12 \mathrm{ft} \times 36 \mathrm{ft}$

EMRC-NISA/DISPLAY JUL/31/06 $12: 14: 38$

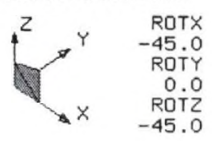

Figure B.4: Fourth mode shape of the isotropic plate (12ft x $36 \mathrm{ft})$ in NISA 

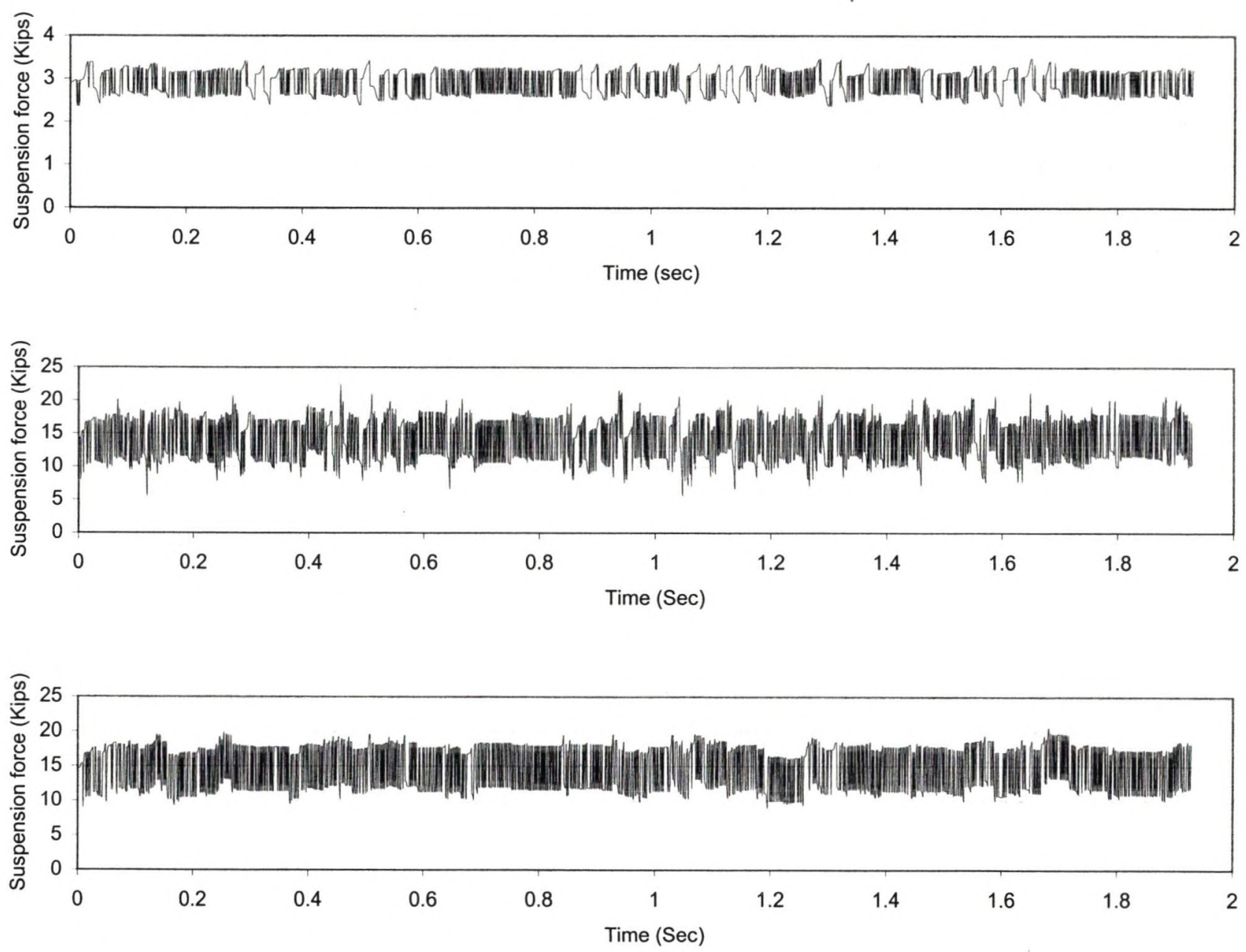

Figure C.1: Suspension force history of steer axle, tractor axle, and trailer axle of an AASHTO HS20-44 truck at speed of $40 \mathrm{mph}$ 

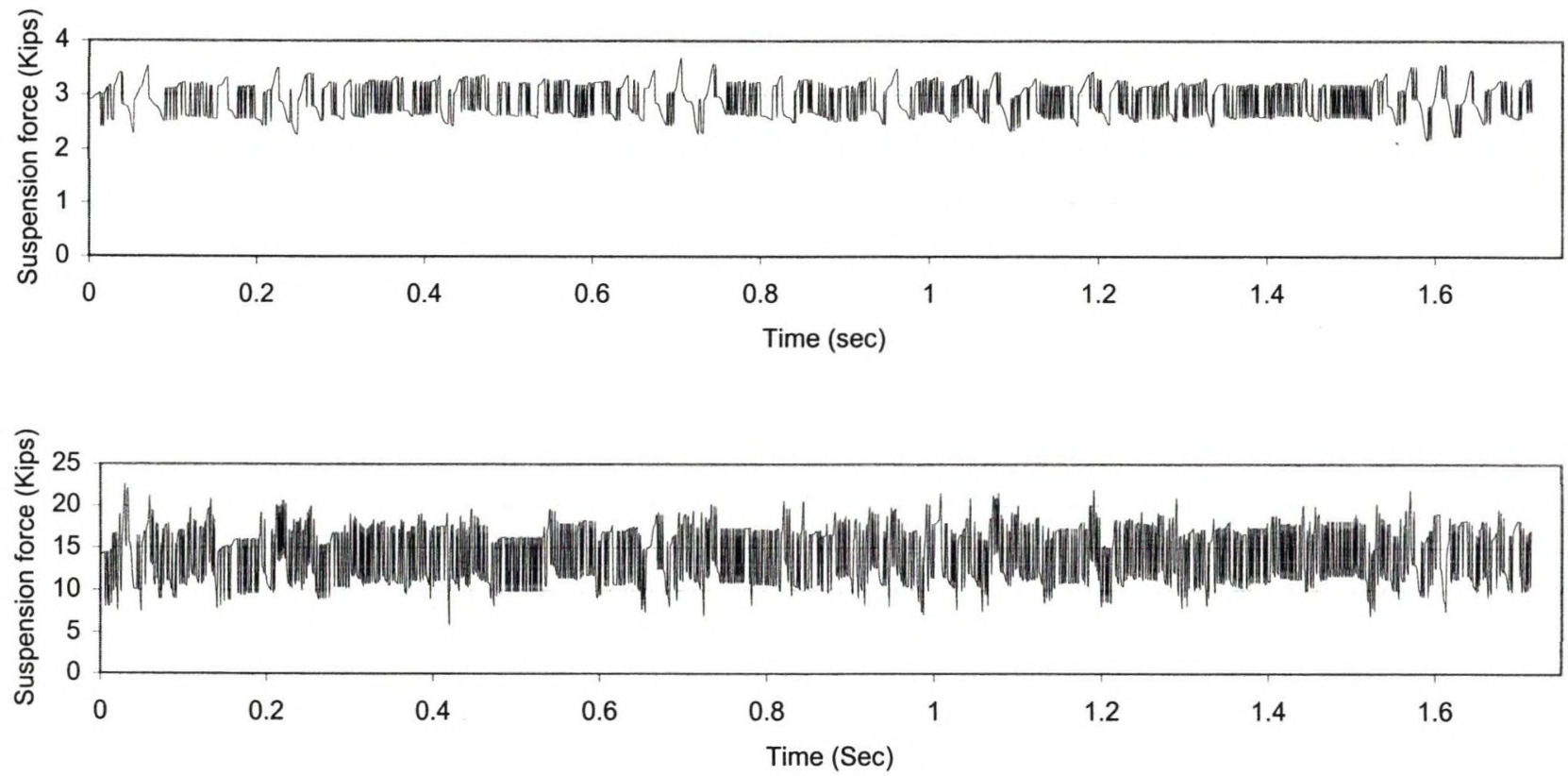

Time (Sec)

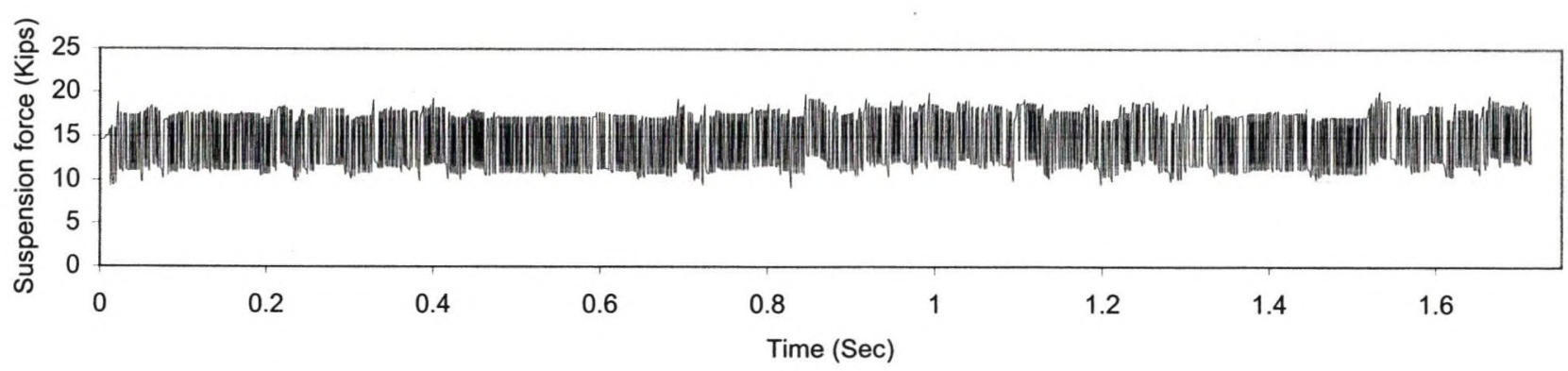

Figure C.2: Suspension force history of steer axle, tractor axle, and trailer axle of an AASHTO HS20-44 truck at speed of $45 \mathrm{mph}$ 

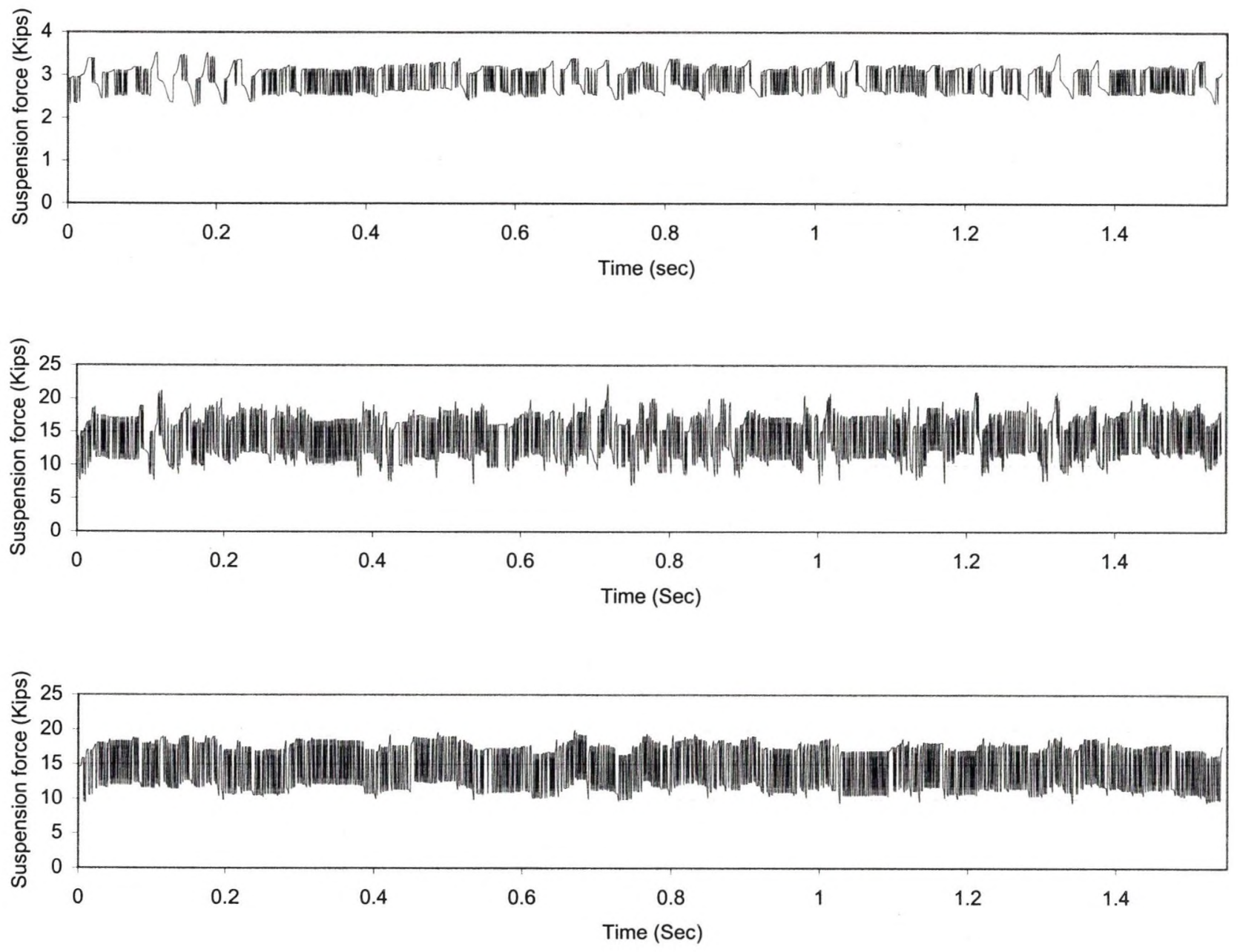

Figure C.3: Suspension force history of steer axle, tractor axle, and trailer axle of an AASHTO HS20-44 truck at speed of $50 \mathrm{mph}$ 

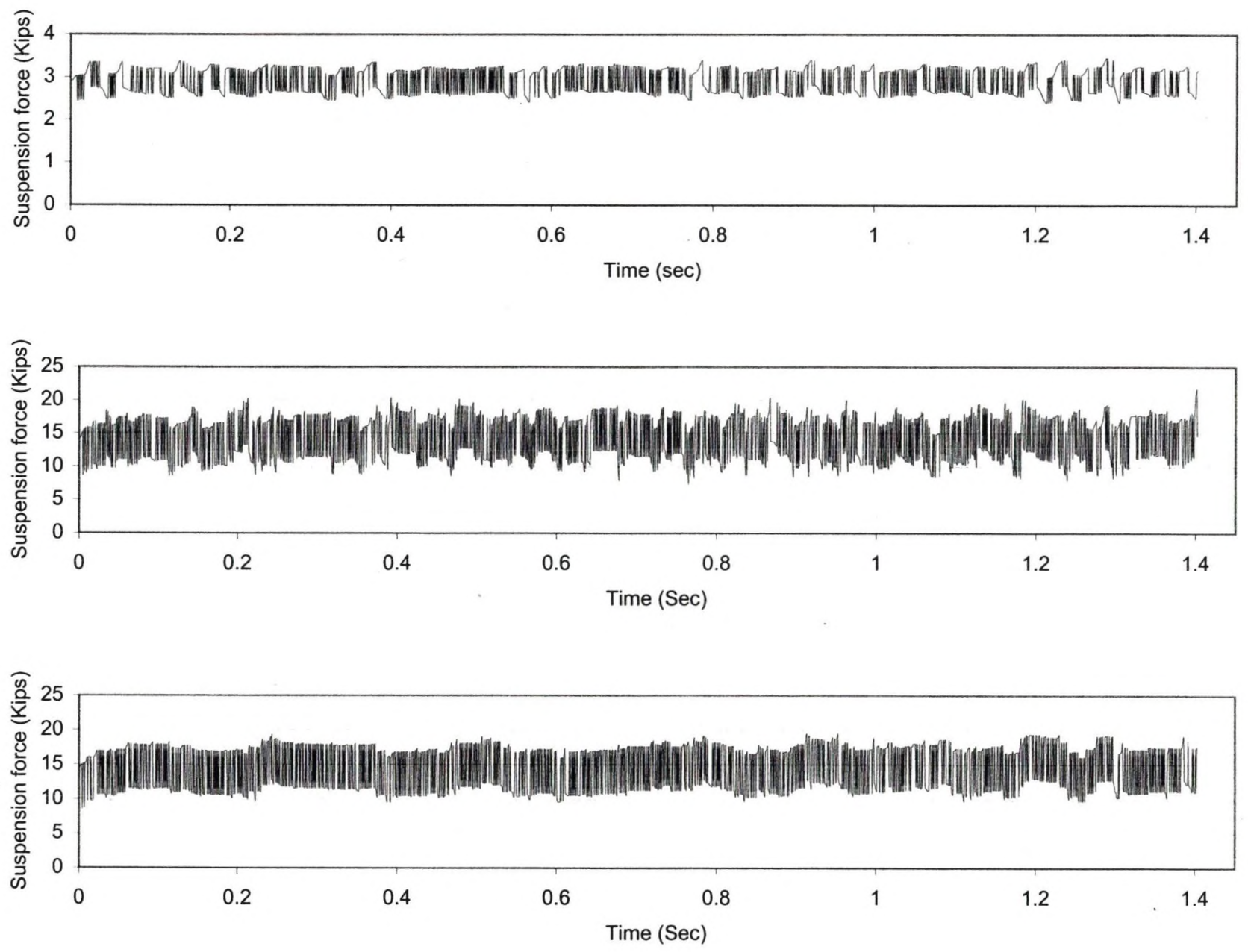

Figure C.4: Suspension force history of steer axle, tractor axle, and trailer axle of an AASHTO HS20-44 truck at speed of $55 \mathrm{mph}$ 

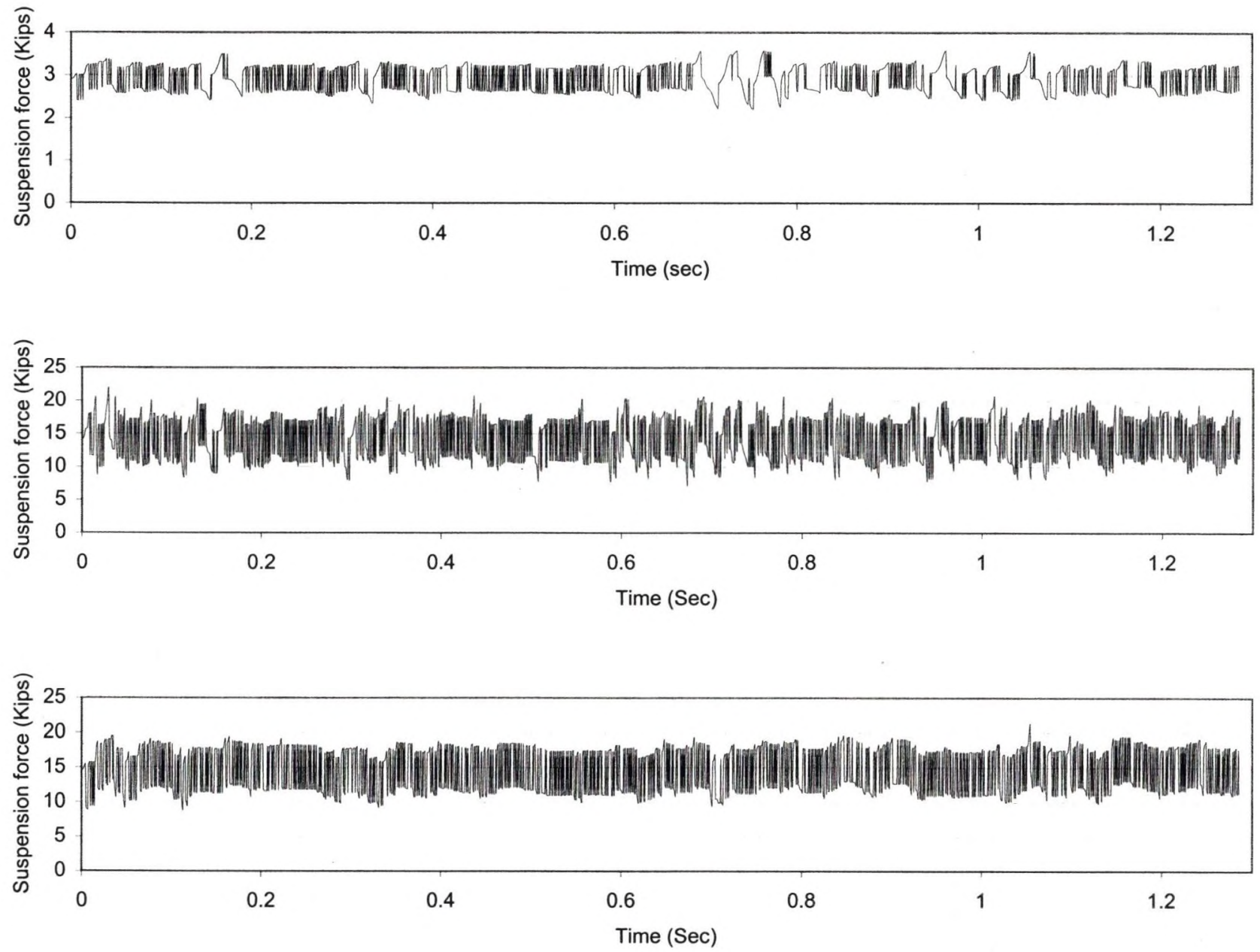

Figure C.5: Suspension force history of steer axle, tractor axle, and trailer axle of an AASHTO HS20-44 truck at speed of $60 \mathrm{mph}$ 


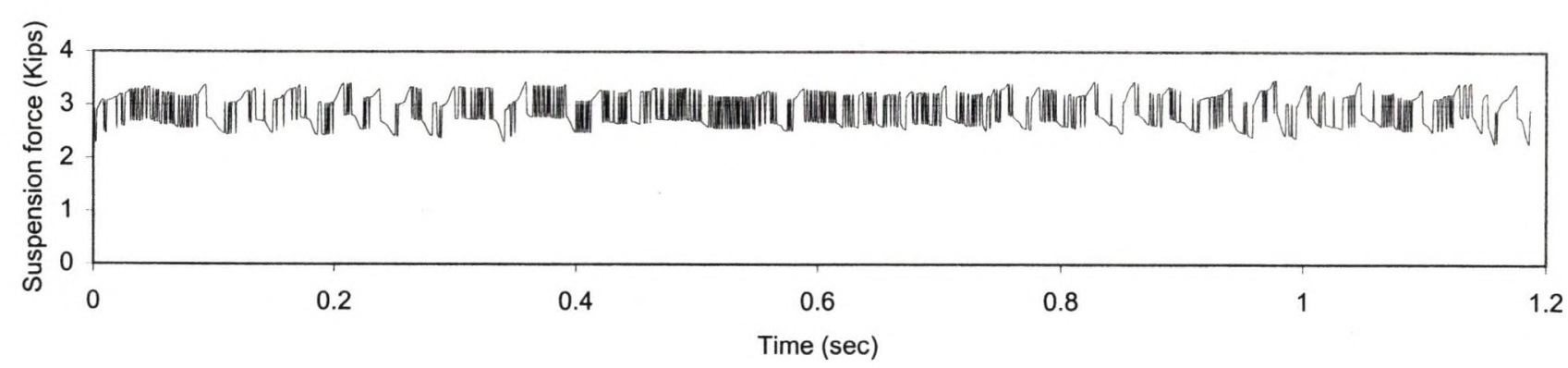

FSY1
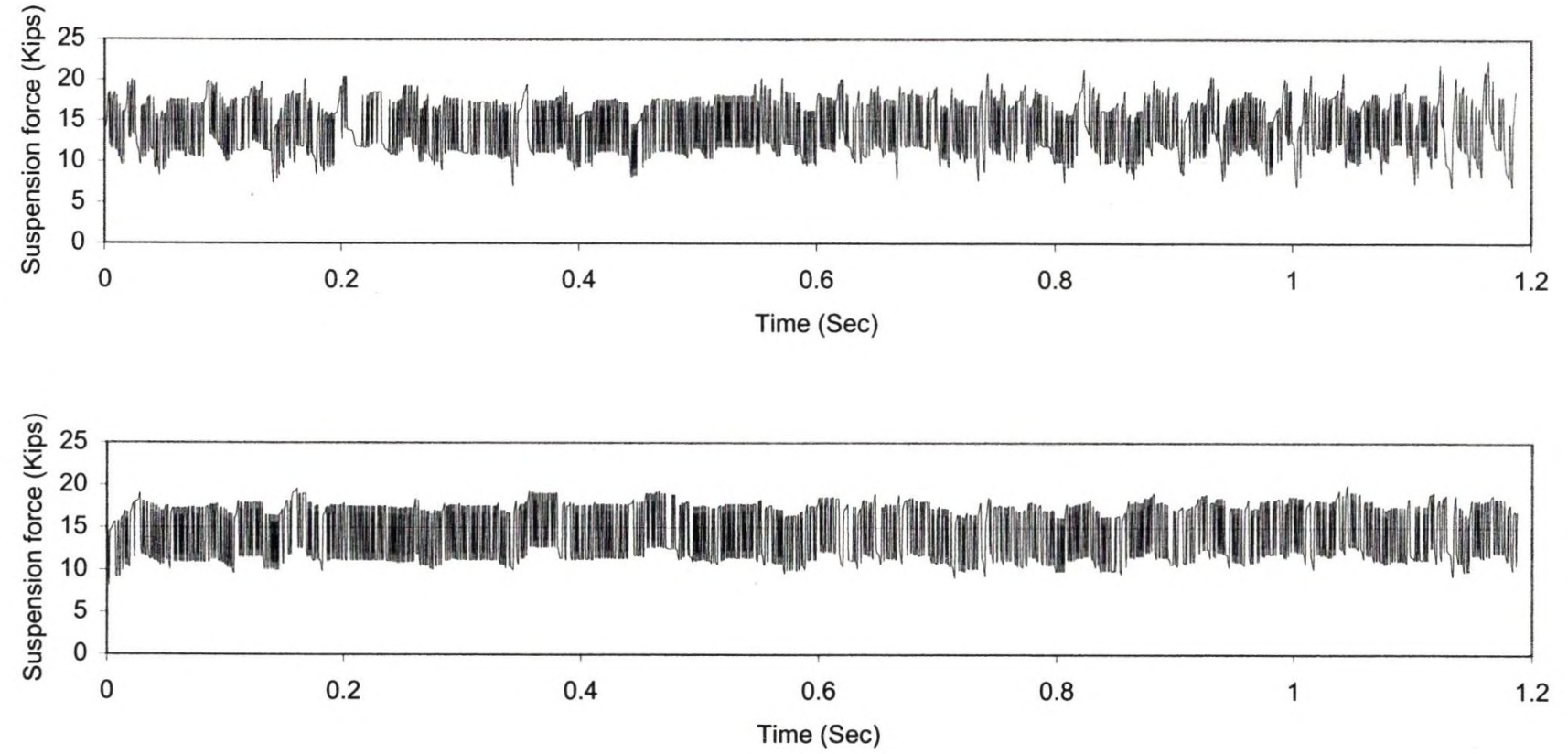

Figure C.6: Suspension force history of steer axle, tractor axle, and trailer axle of an AASHTO HS20-44 truck at speed of $65 \mathrm{mph}$ 

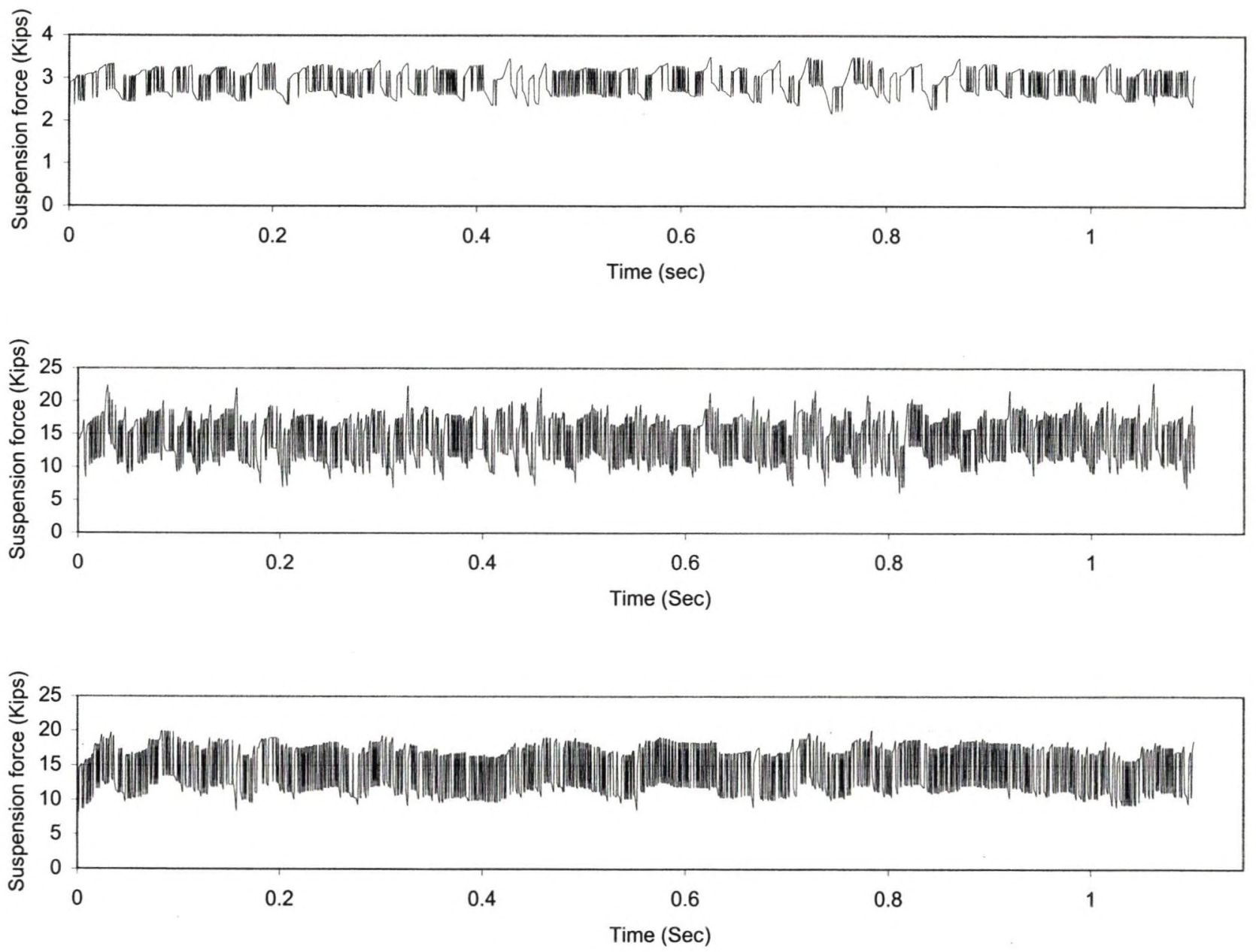

Figure C.7: Suspension force history of steer axle, tractor axle, and trailer axle of an AASHTO HS20-44 truck at speed of $70 \mathrm{mph}$ 

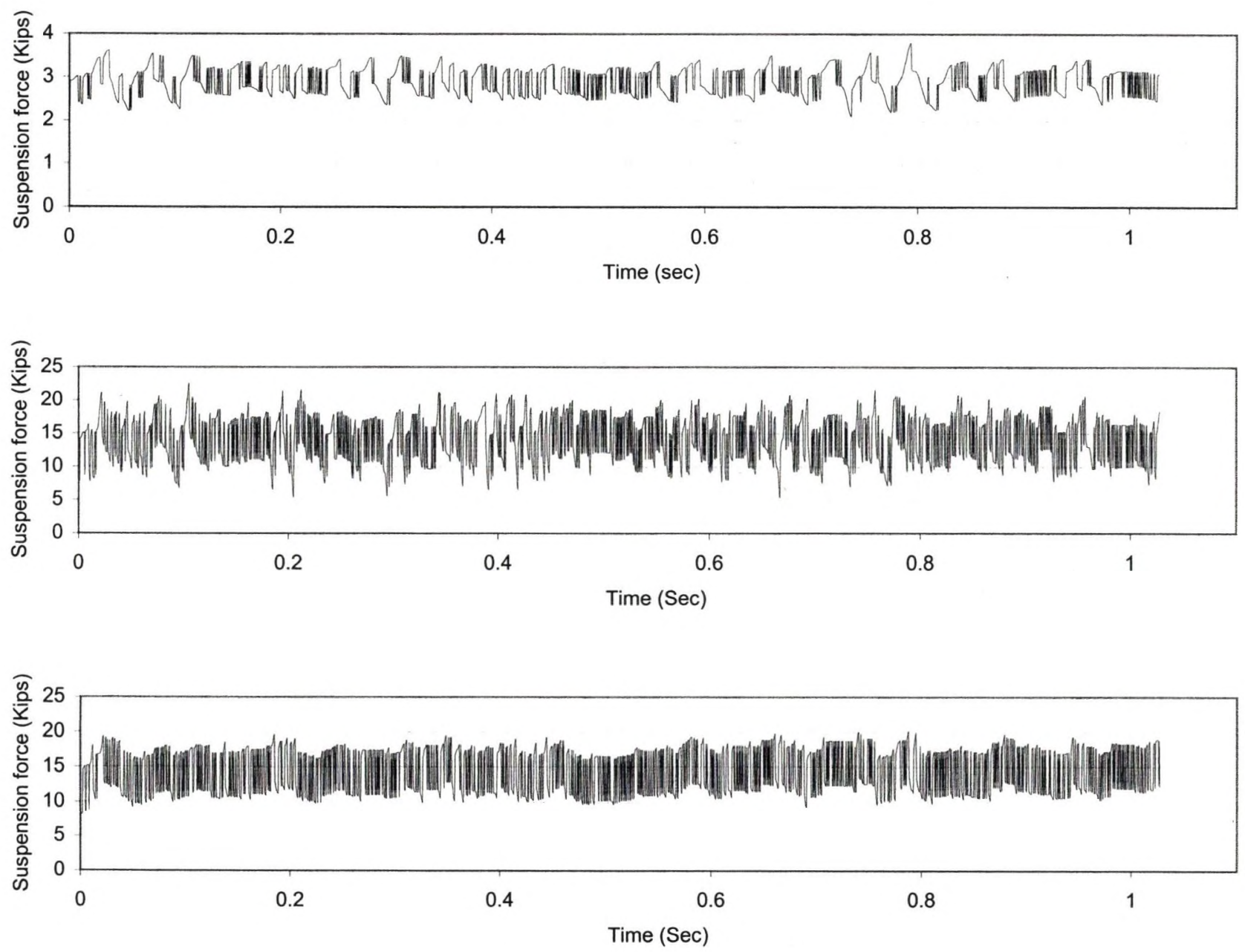

Figure C.8: Suspension force history of steer axle, tractor axle, and trailer axle of an AASHTO HS20-44 truck at speed of $75 \mathrm{mph}$ 

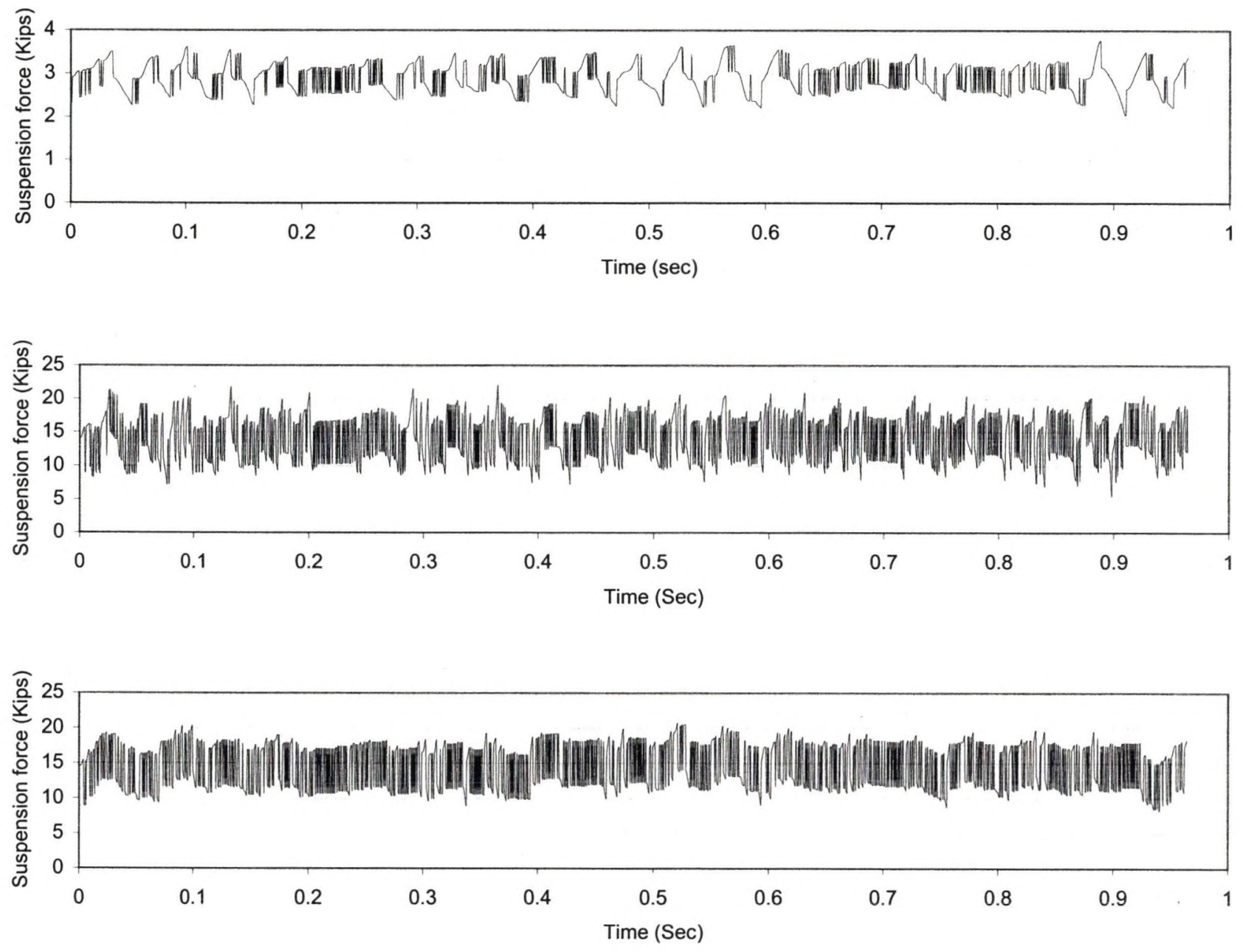

Figure C.9: Suspension force history of steer axle, tractor axle, and trailer axle of an AASHTO HS20-44 truck at speed of $80 \mathrm{mph}$ 

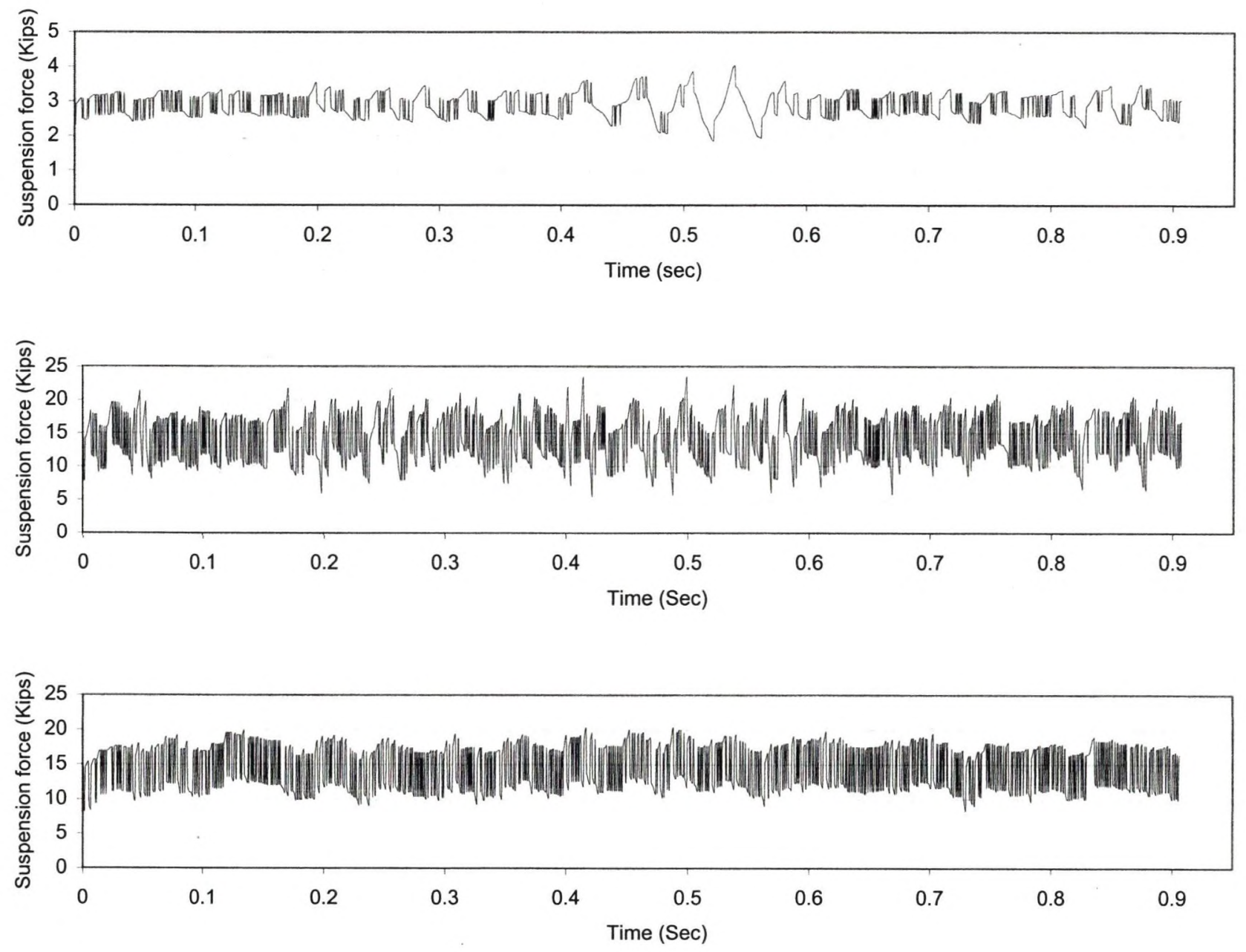

Figure C.10: Suspension force history of steer axle, tractor axle, and trailer axle of an AASHTO HS20-44 truck at speed of $85 \mathrm{mph}$ 


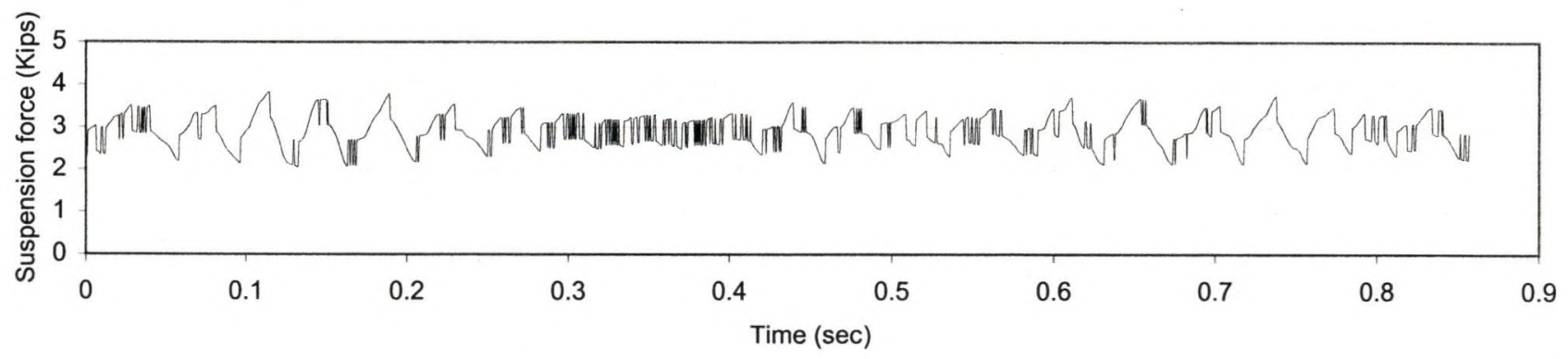

FSY1
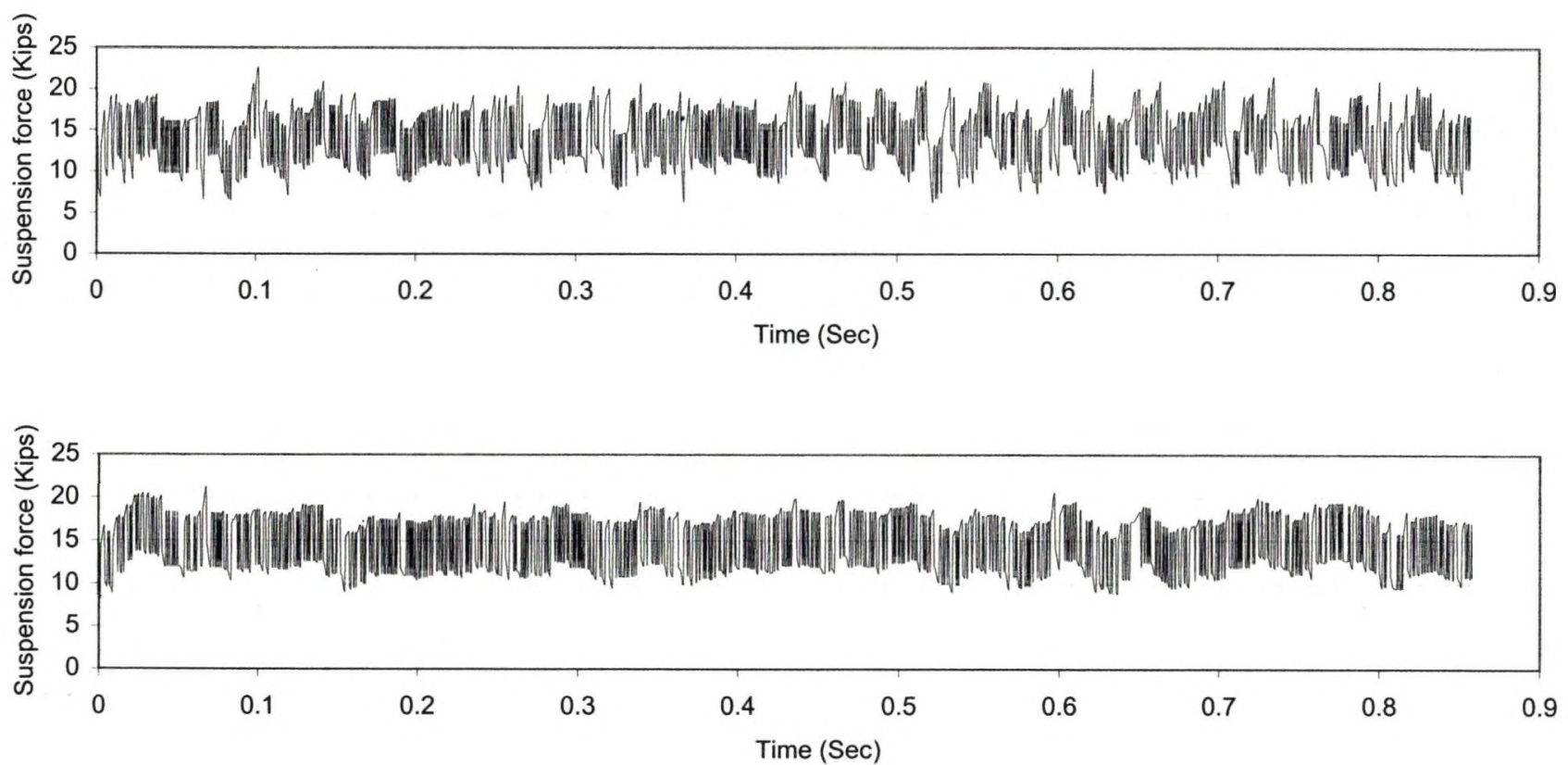

Figure C.11: Suspension force history of steer axle, tractor axle, and trailer axle of an AASHTO HS20-44 truck at speed of $90 \mathrm{mph}$ 

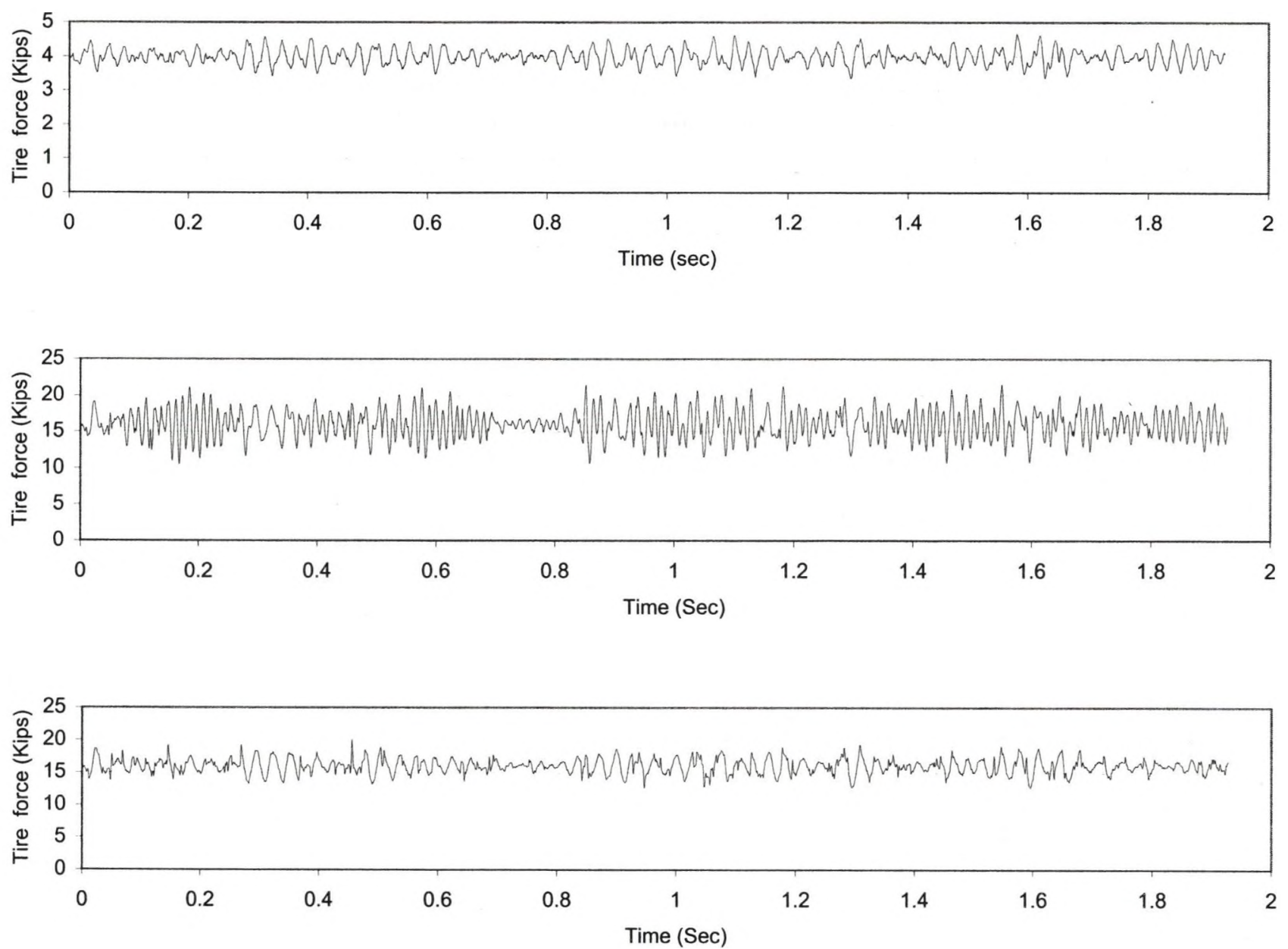

Figure C.12: Tire force history of steer axle, tractor axle, and trailer axle of an AASHTO HS20-44 truck at speed of $40 \mathrm{mph}$ 

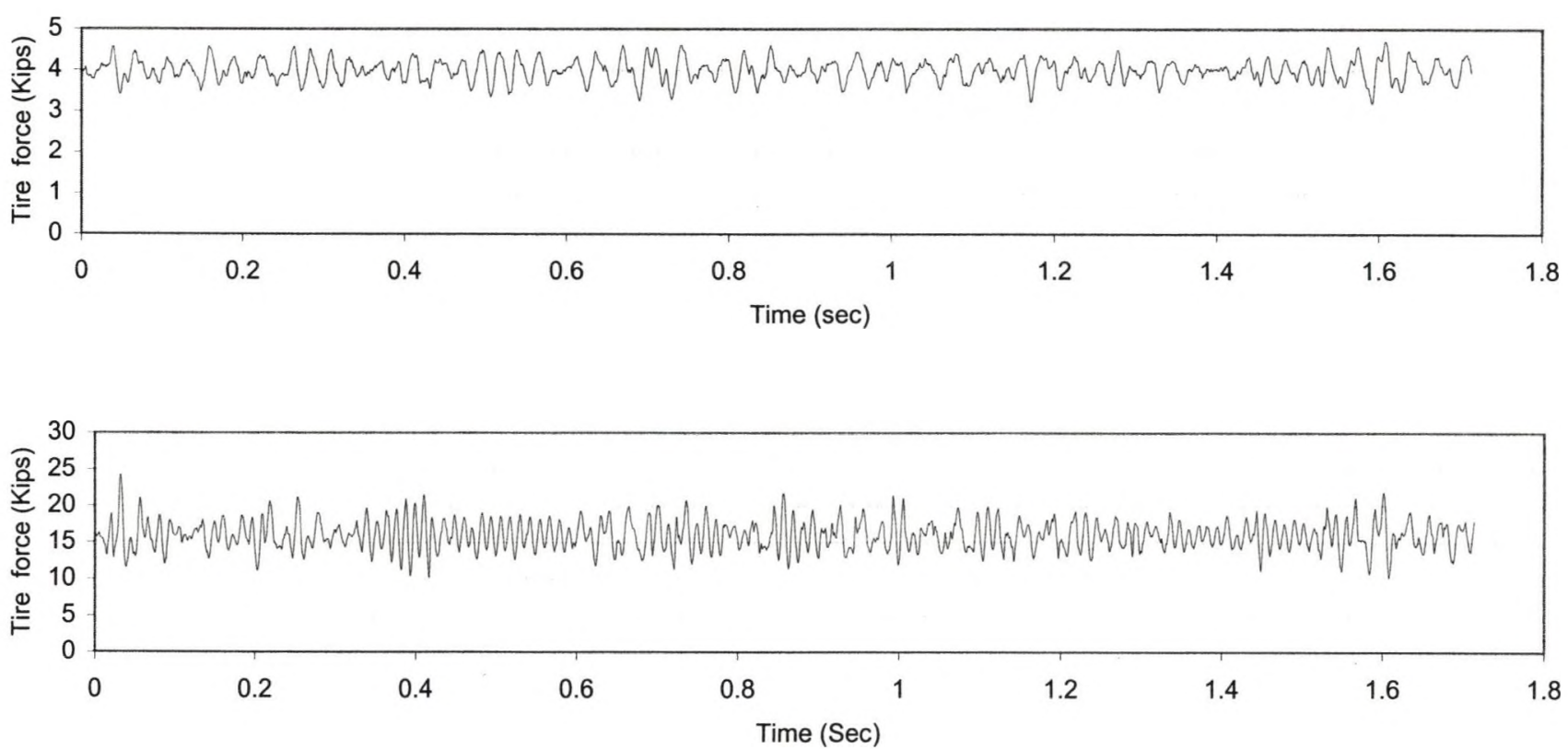

Time (Sec)

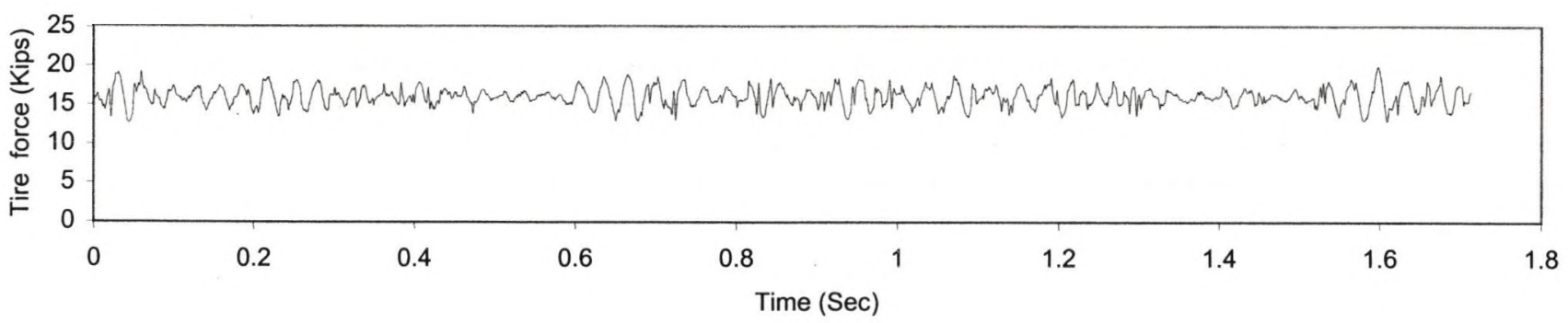

Figure C.13: Tire force history of steer axle, tractor axle, and trailer axle of an AASHTO HS20-44 truck at speed of $45 \mathrm{mph}$ 

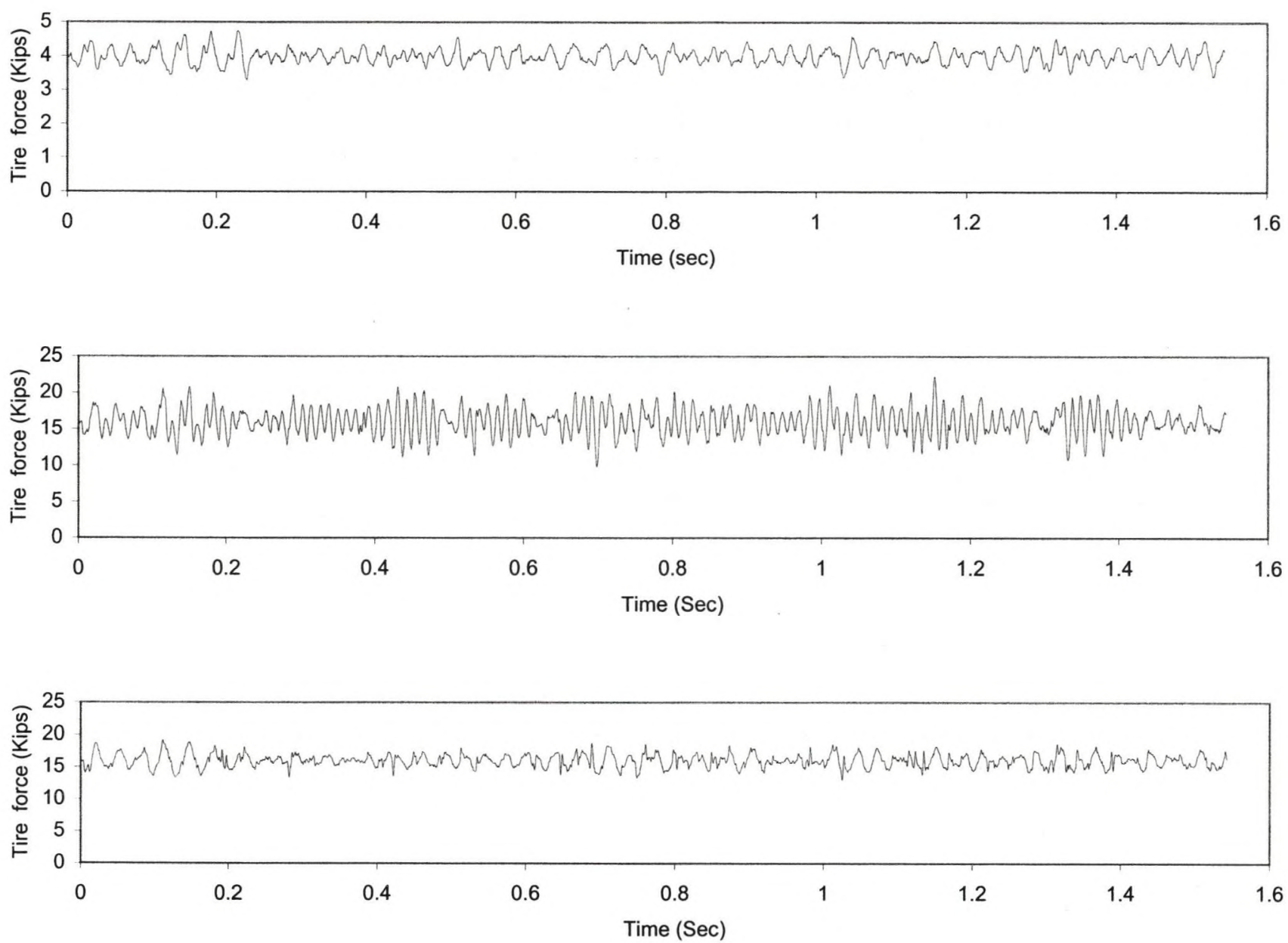

Figure C.14: Tire force history of steer axle, tractor axle, and trailer axle of an AASHTO HS20-44 truck at speed of $50 \mathrm{mph}$ 

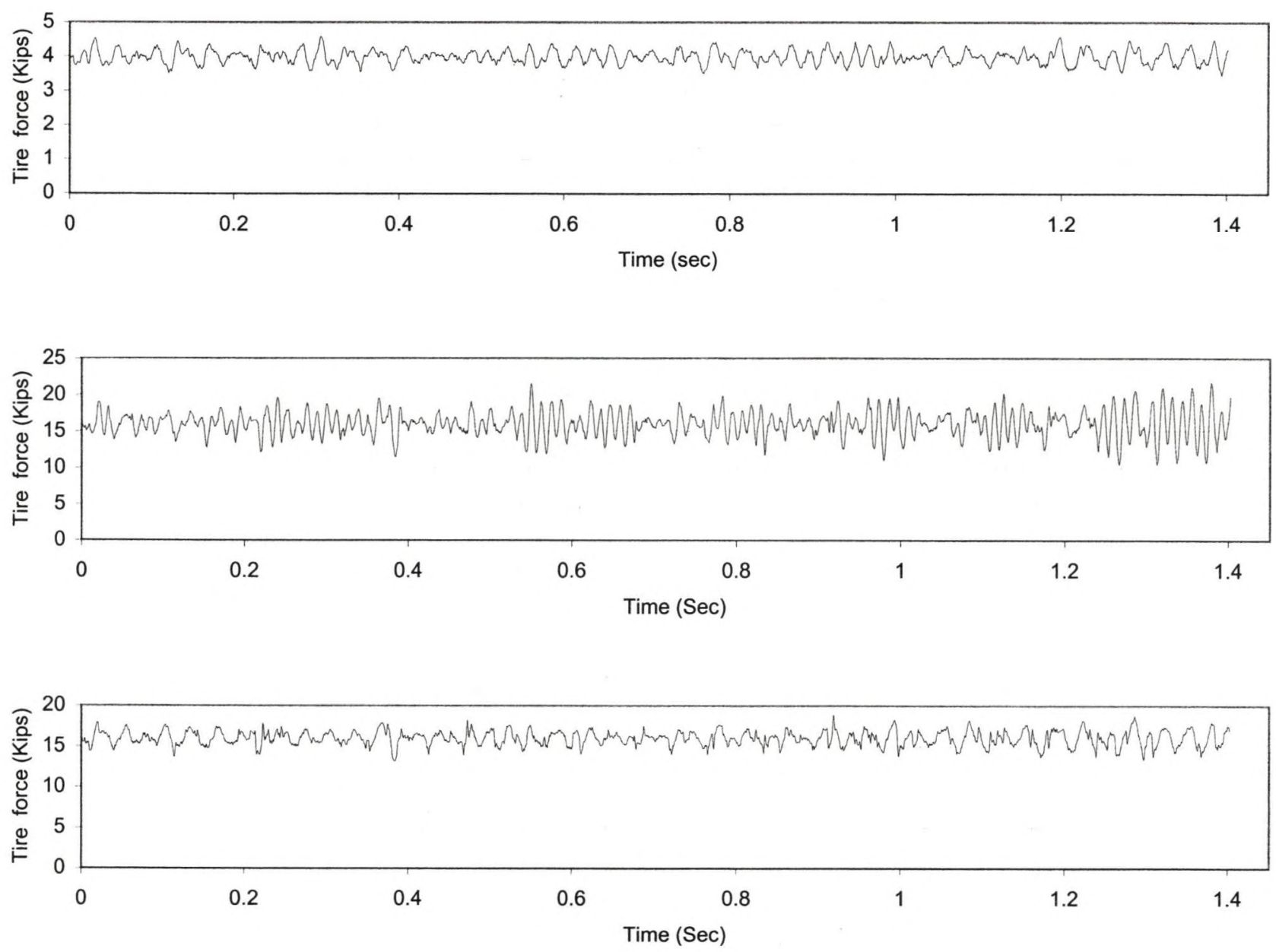

Figure C.15: Tire force history of steer axle, tractor axle, and trailer axle of an AASHTO HS20-44 truck at speed of $55 \mathrm{mph}$ 

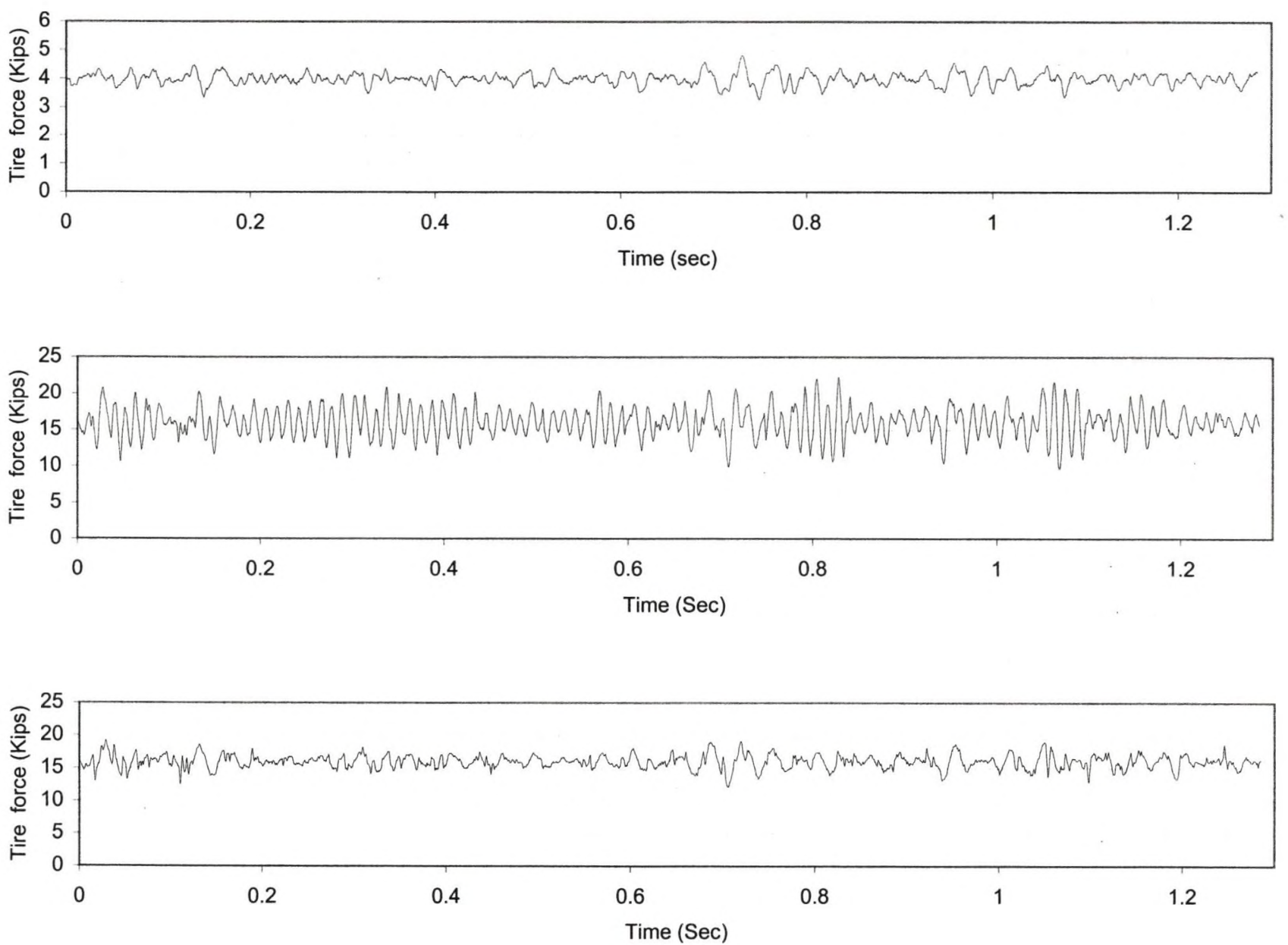

Figure C.16: Tire force history of steer axle, tractor axle, and trailer axle of an AASHTO HS20-44 truck at speed of $60 \mathrm{mph}$ 

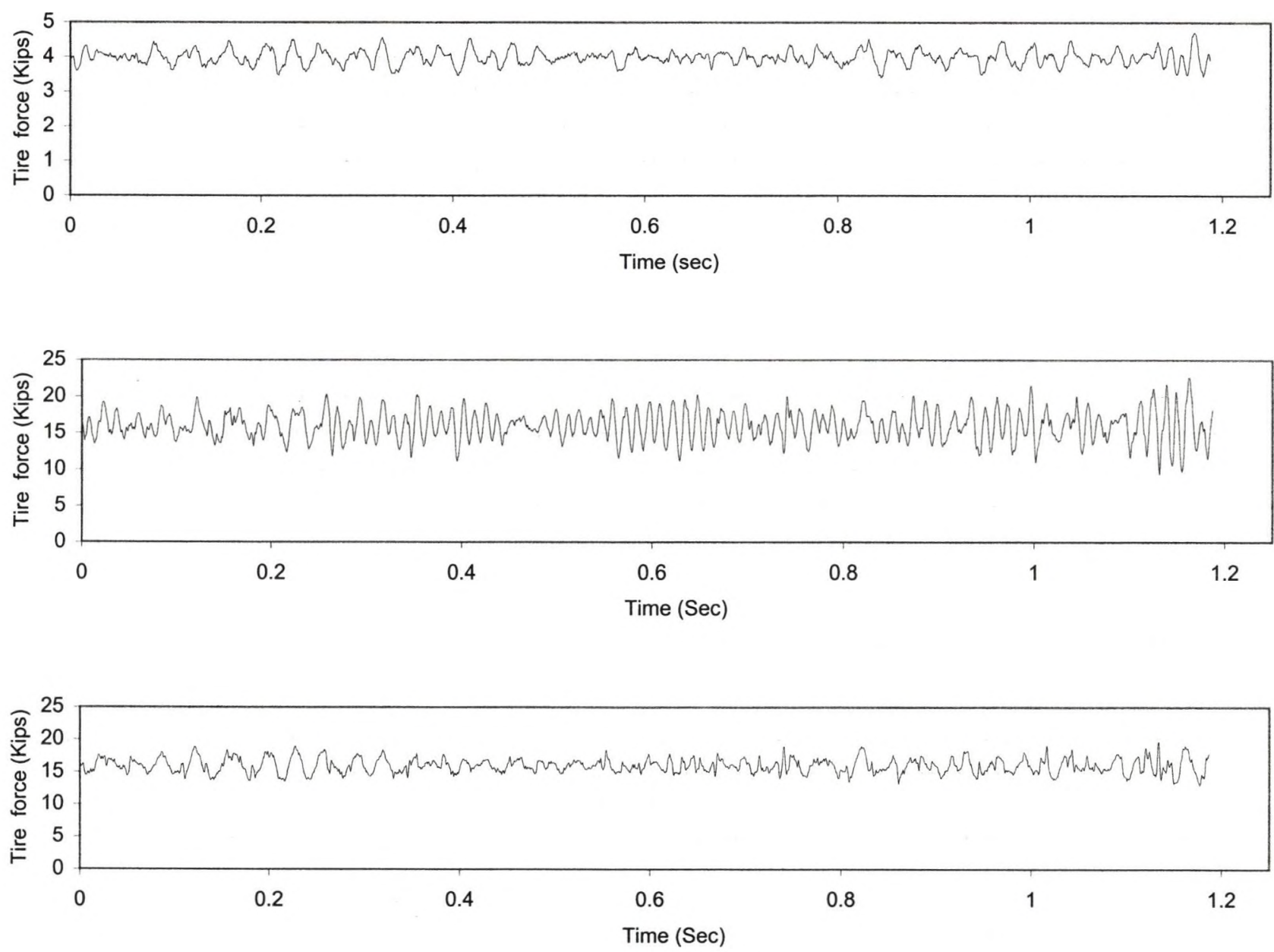

Figure C.17: Tire force history of steer axle, tractor axle, and trailer axle of an AASHTO HS20-44 truck at speed of $65 \mathrm{mph}$ 

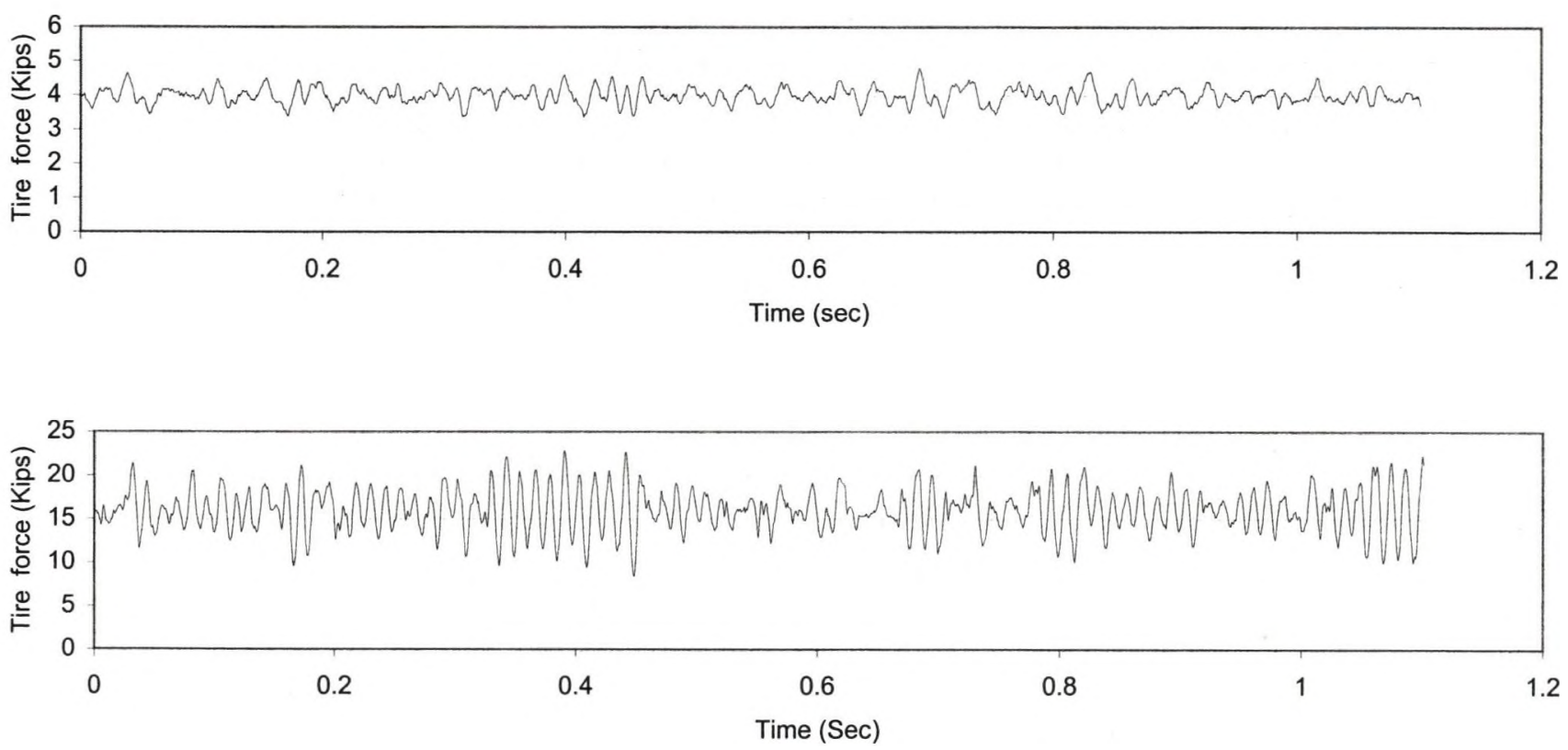

Time (Sec)

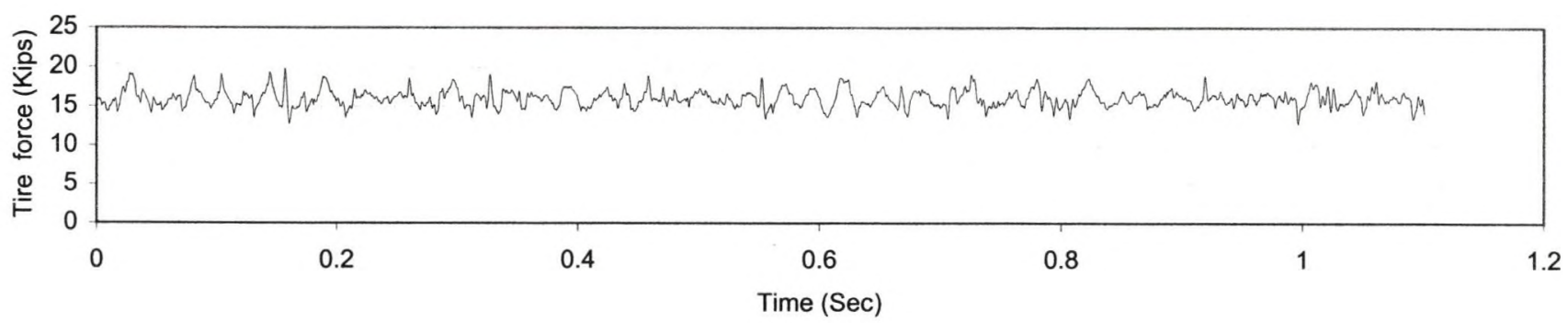

Figure C.18: Tire force history of steer axle, tractor axle, and trailer axle of an AASHTO HS20-44 truck at speed of $70 \mathrm{mph}$ 

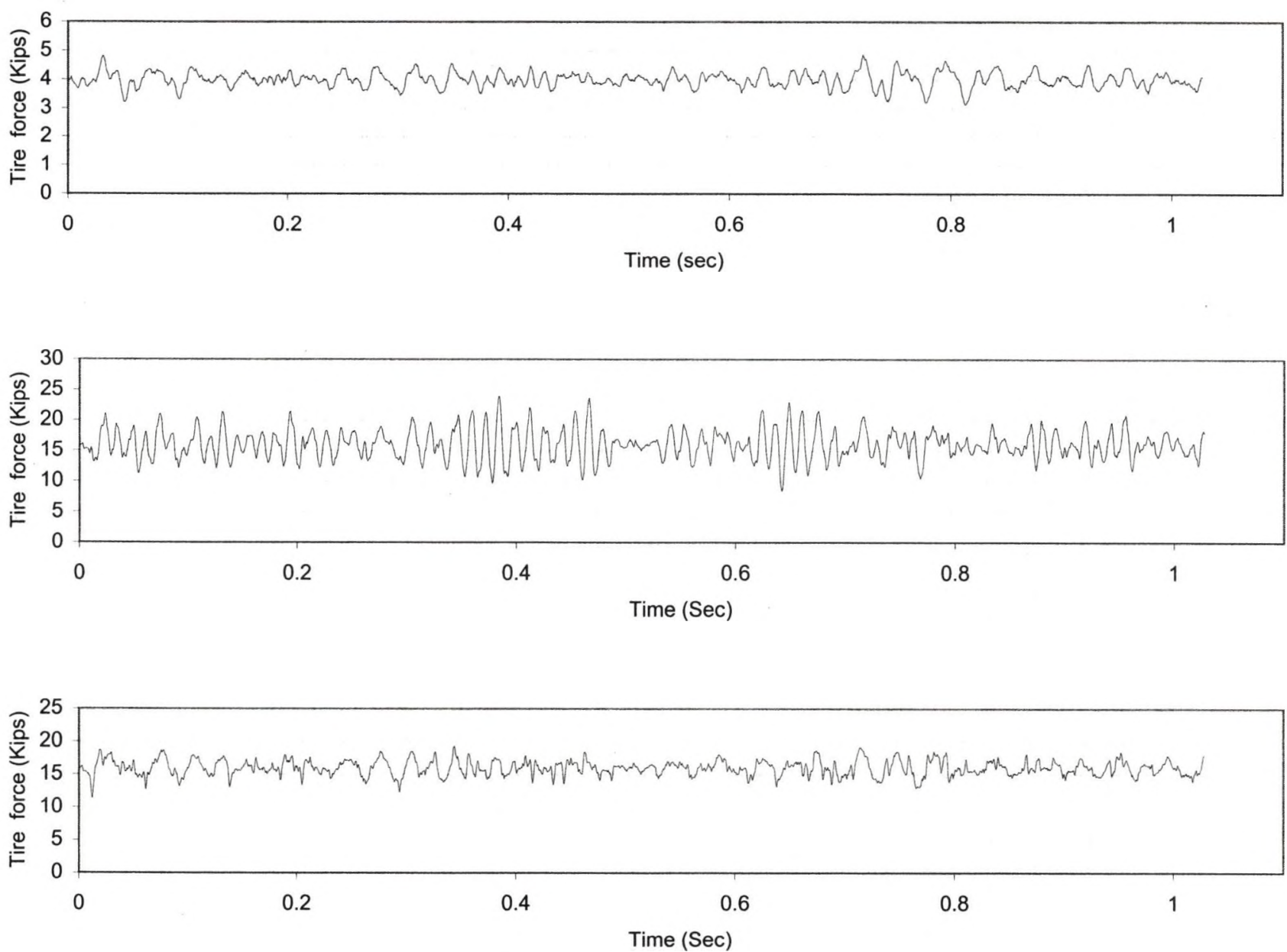

Figure C.19: Tire force history of steer axle, tractor axle, and trailer axle of an AASHTO HS20-44 truck at speed of $75 \mathrm{mph}$ 

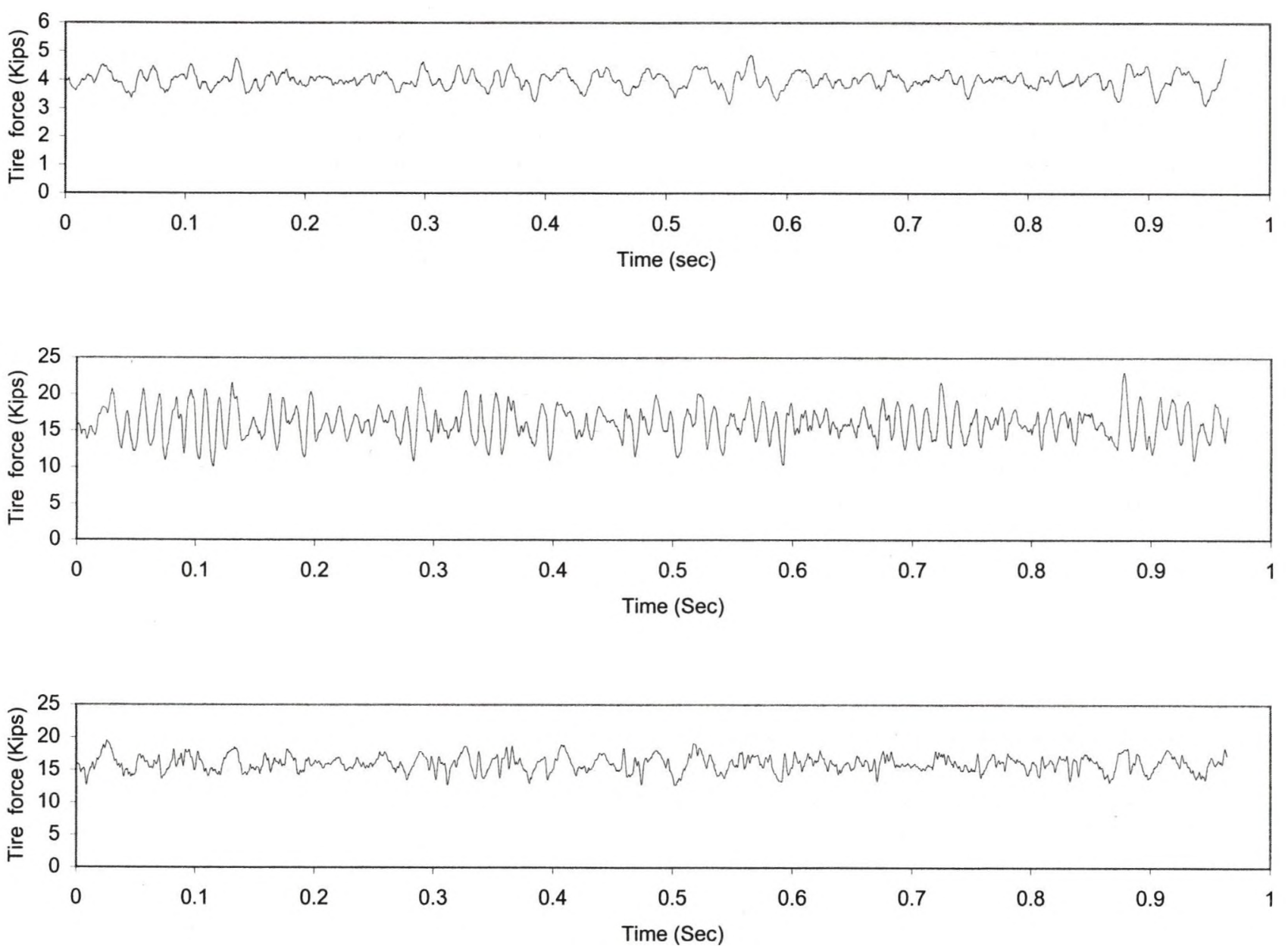

Figure C.20: Tire force history of steer axle, tractor axle, and trailer axle of an AASHTO HS20-44 truck at speed of $80 \mathrm{mph}$ 

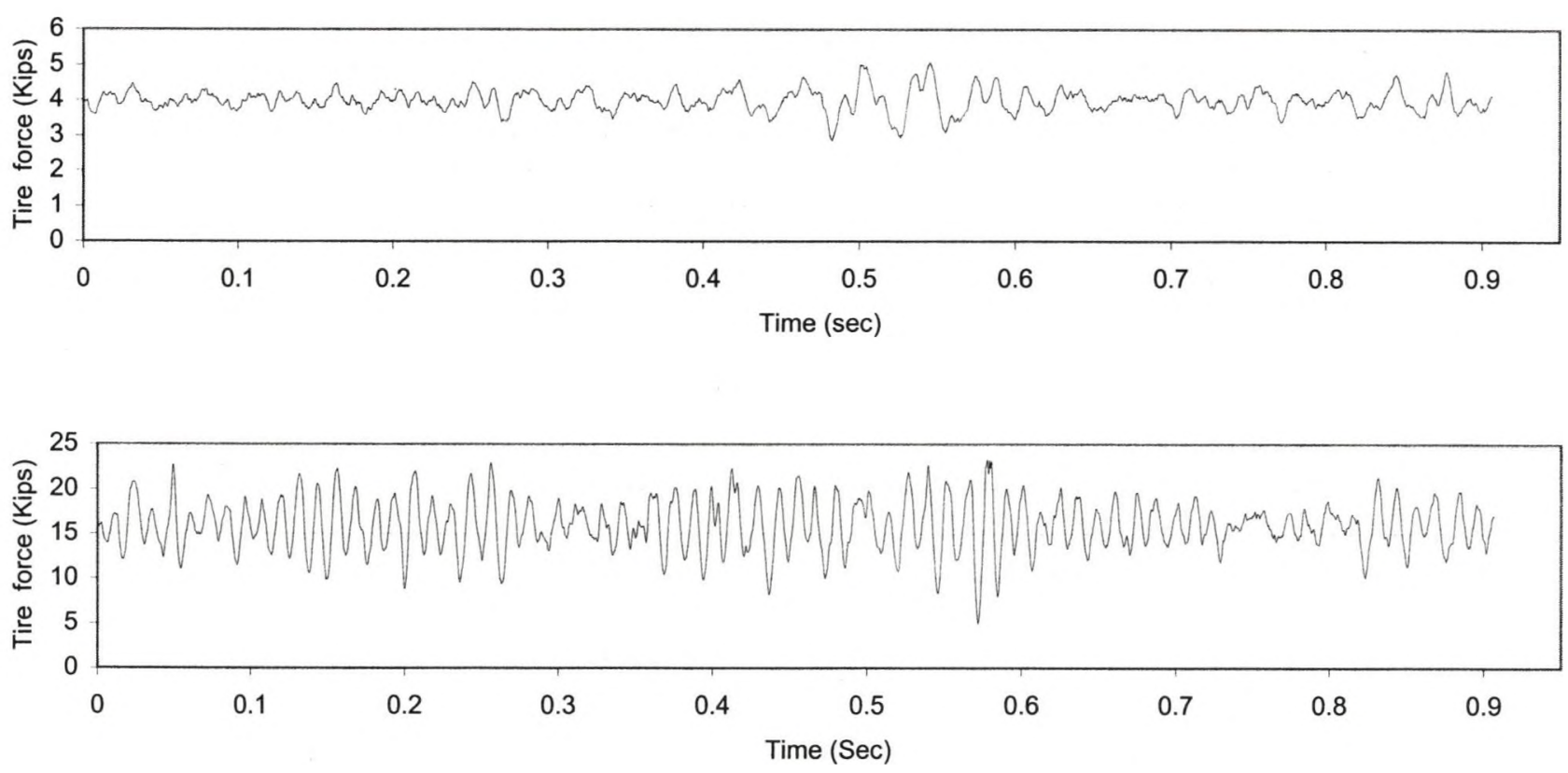

Time (Sec)

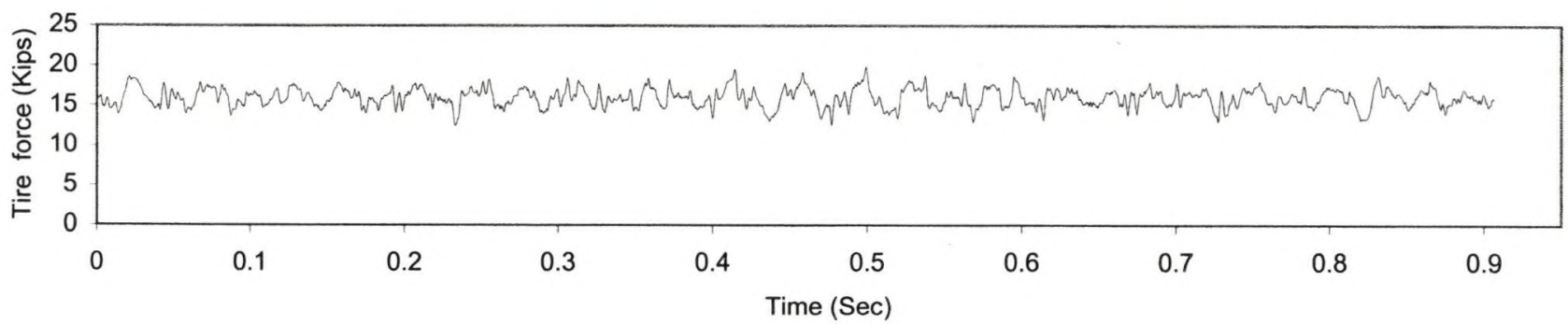

Figure C.21: Tire force history of steer axle, tractor axle, and trailer axle of an AASHTO HS20-44 truck at speed of $85 \mathrm{mph}$ 

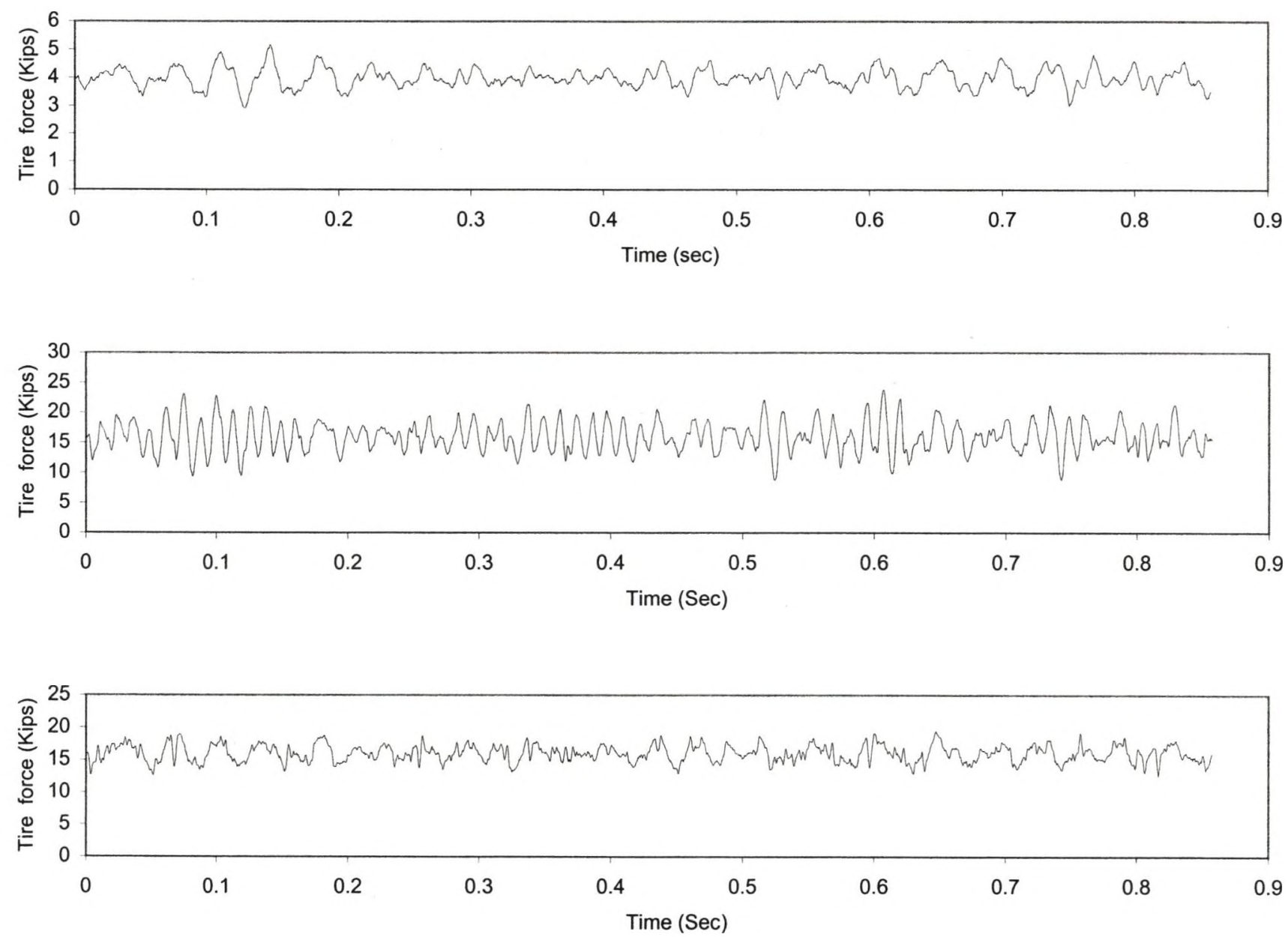

Figure C.22: Tire force history of steer axle, tractor axle, and trailer axle of an AASHTO HS20-44 truck at speed of $90 \mathrm{mph}$ 


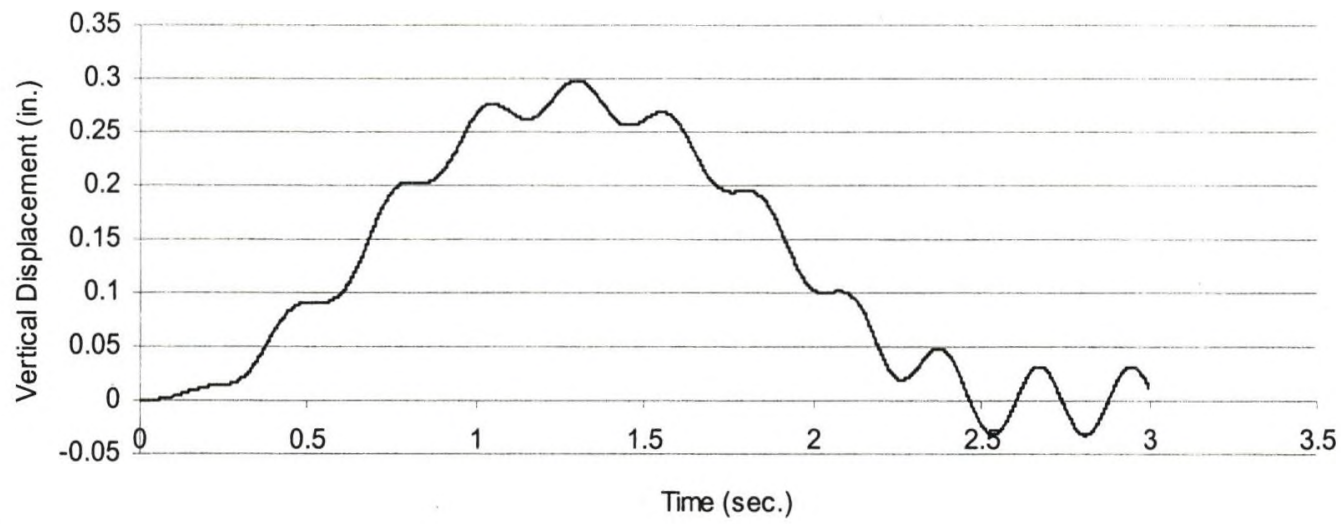

Figures C.23: Time history of the vertical displacement at the center of the T-beam bridge deck under an AASHTO HS20-44 truck with a speed of $40 \mathrm{mph}$.

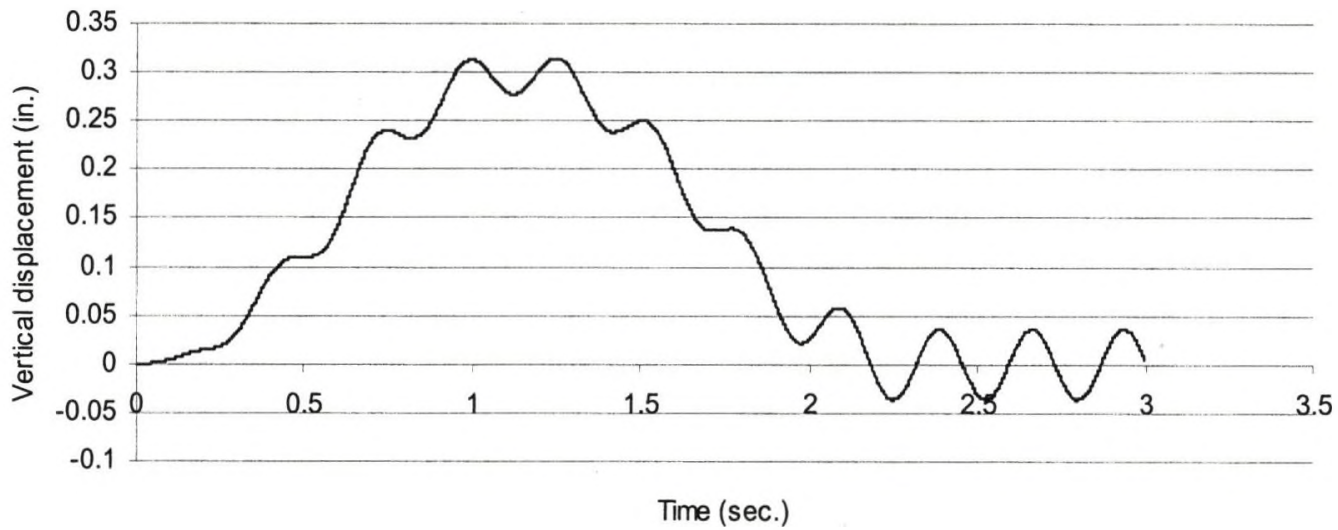

Figures C.24: Time history of the vertical displacement at the center of the T-beam bridge deck under an AASHTO HS20-44 truck with a speed of $45 \mathrm{mph}$.

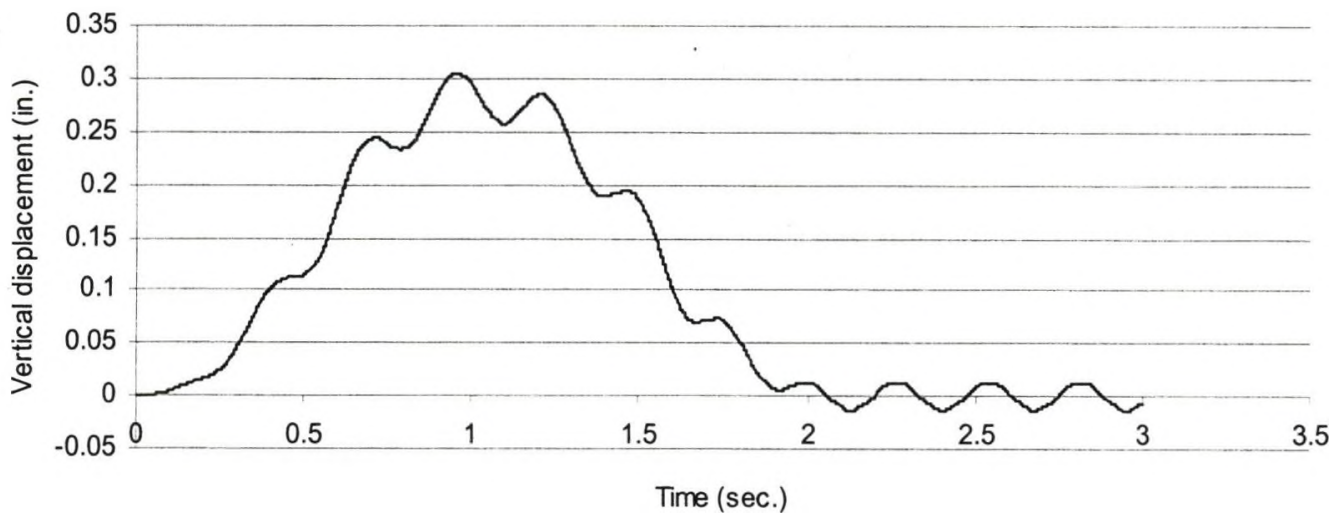

Figures C.25: Time history of the vertical displacement at the center of the T-beam bridge deck under an AASHTO HS20-44 truck with a speed of $50 \mathrm{mph}$. 


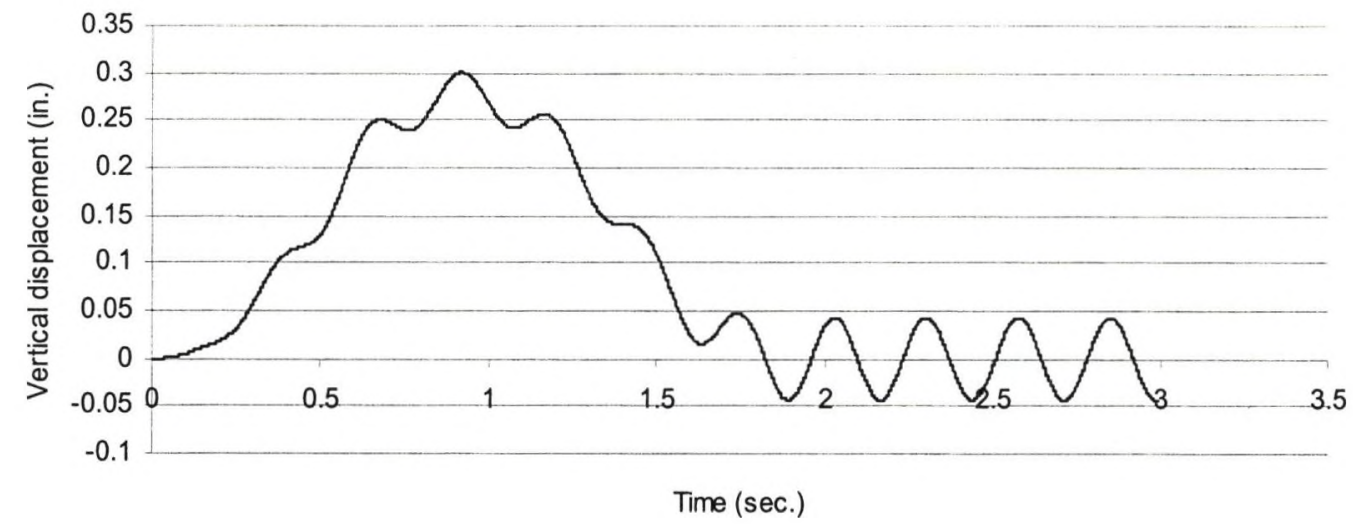

Figures C.26: Time history of the vertical displacement at the center of the T-beam bridge deck under an AASHTO HS20-44 truck with a speed of $55 \mathrm{mph}$

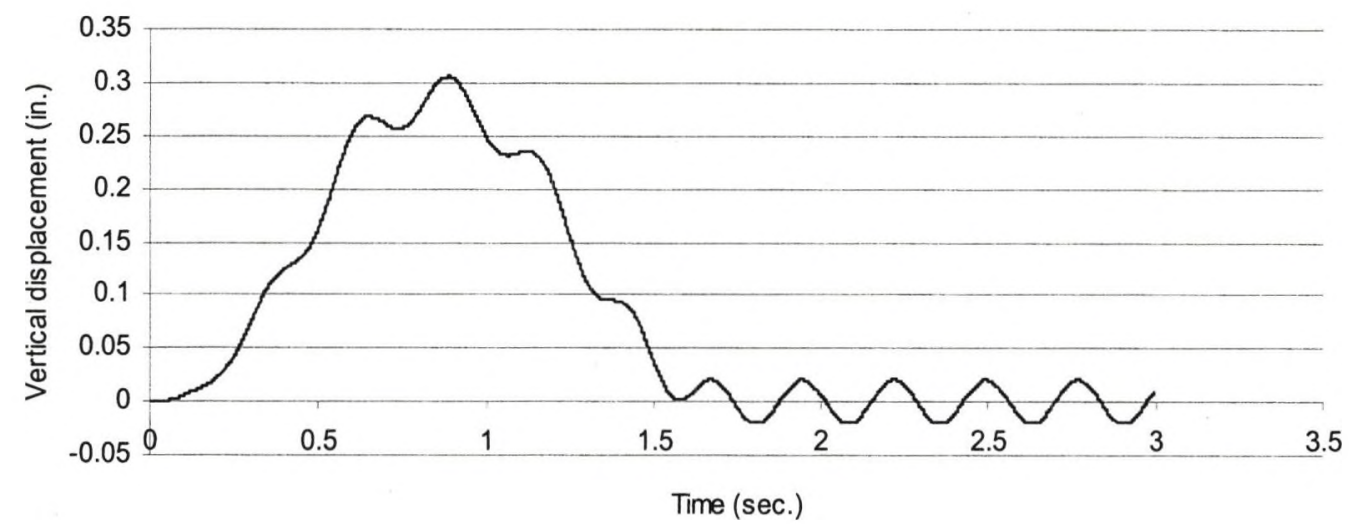

Figures C.27: Time history of the vertical displacement at the center of the T-beam bridge deck under an AASHTO HS20-44 truck with a speed of $60 \mathrm{mph}$

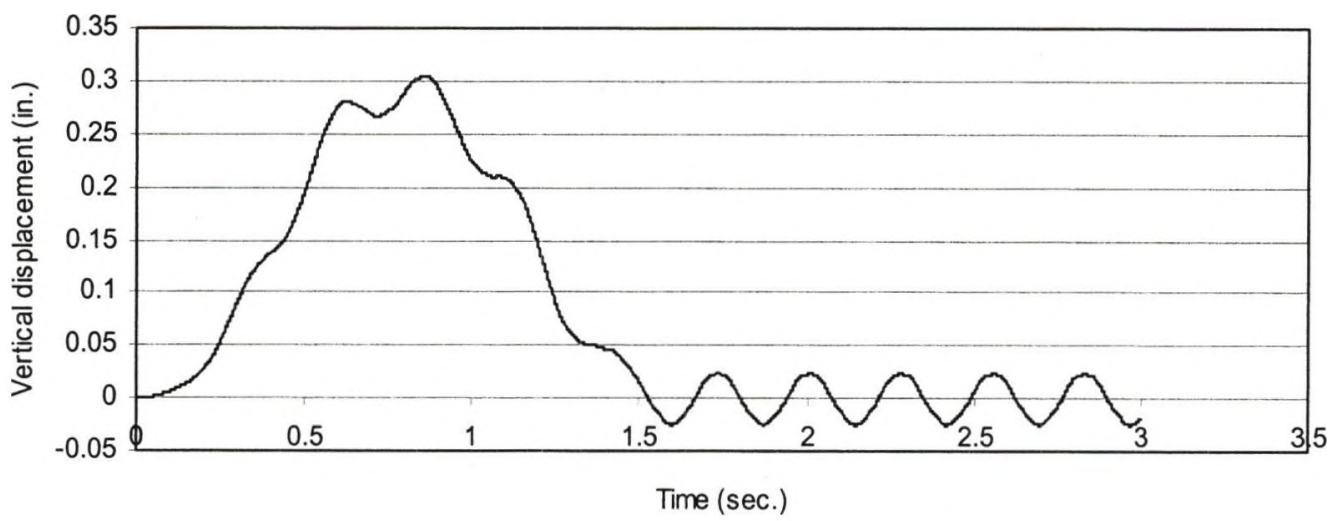

Figures C.28: Time history of the vertical displacement at the center of the T-beam bridge deck under an AASHTO HS20-44 truck with a speed of $65 \mathrm{mph}$ 


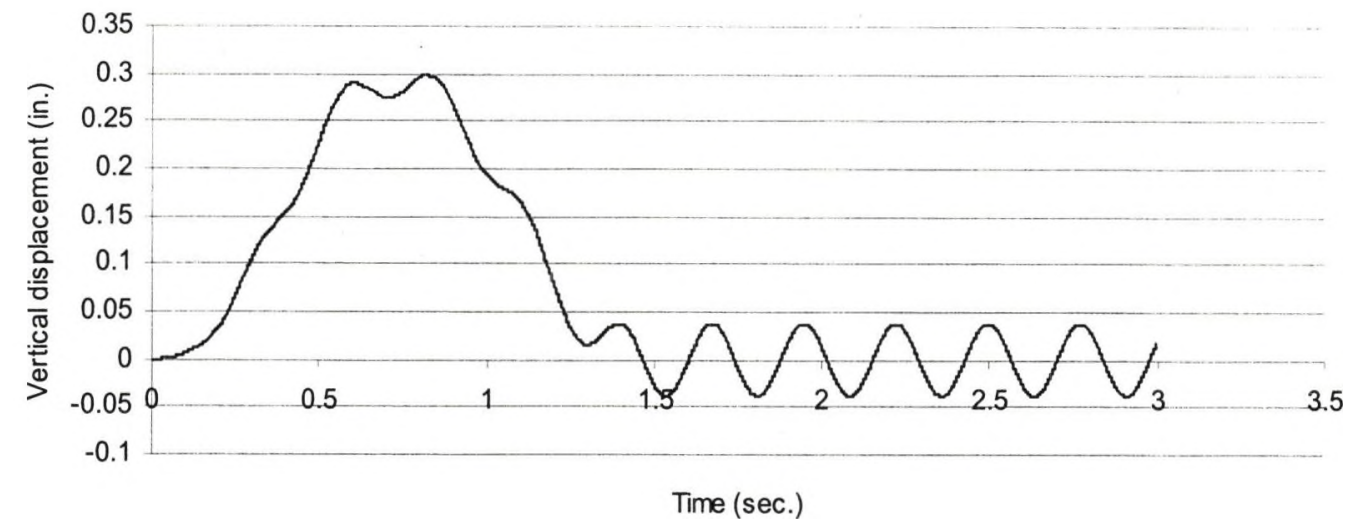

Figures C.29: Time history of the vertical displacement at the center of the T-beam bridge deck under an AASHTO HS20-44 truck with a speed of $70 \mathrm{mph}$

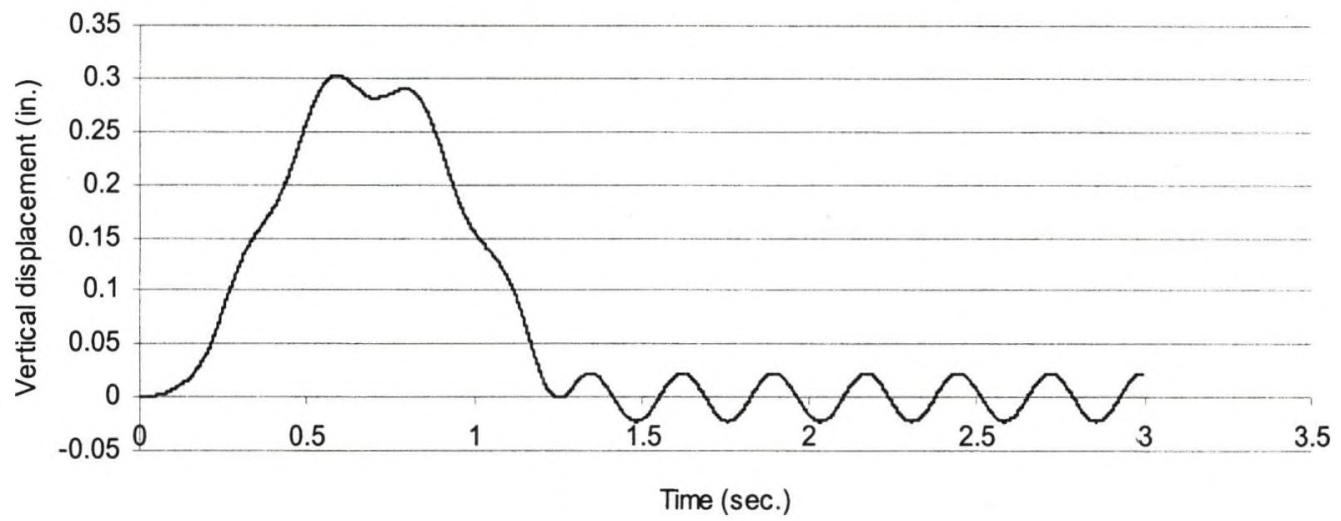

Figures C.30: Time history of the vertical displacement at the center of the T-beam bridge deck under an AASHTO HS20-44 truck with a speed of $75 \mathrm{mph}$

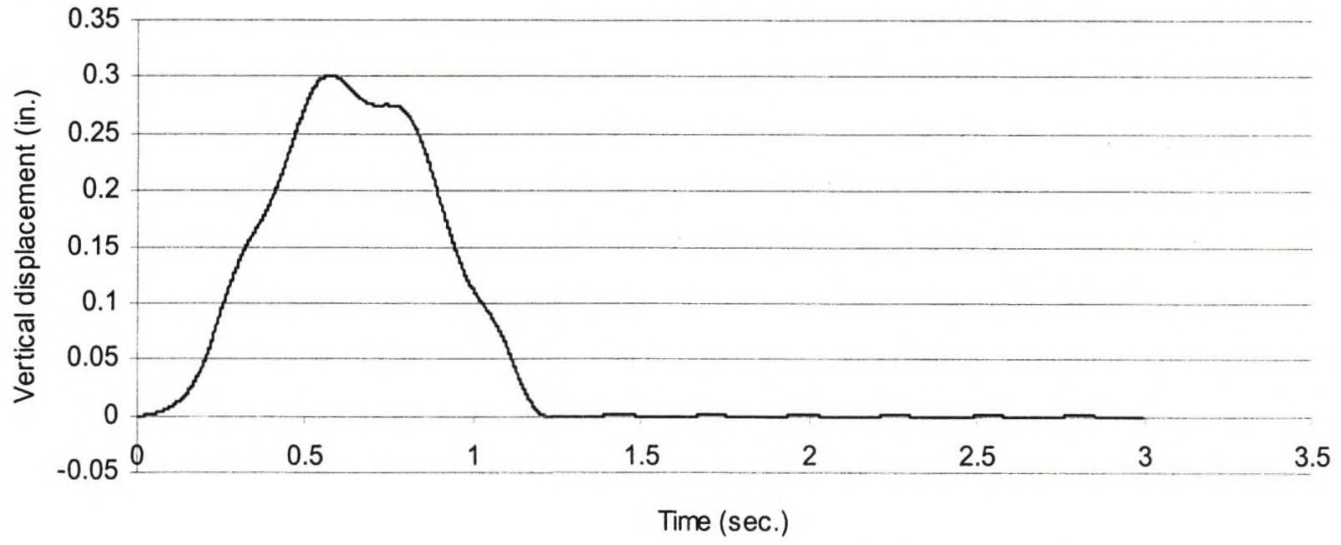

Figures C.31: Time history of the vertical displacement at the center of the T-beam bridge deck under an AASHTO HS20-44 truck with a speed of $80 \mathrm{mph}$ 


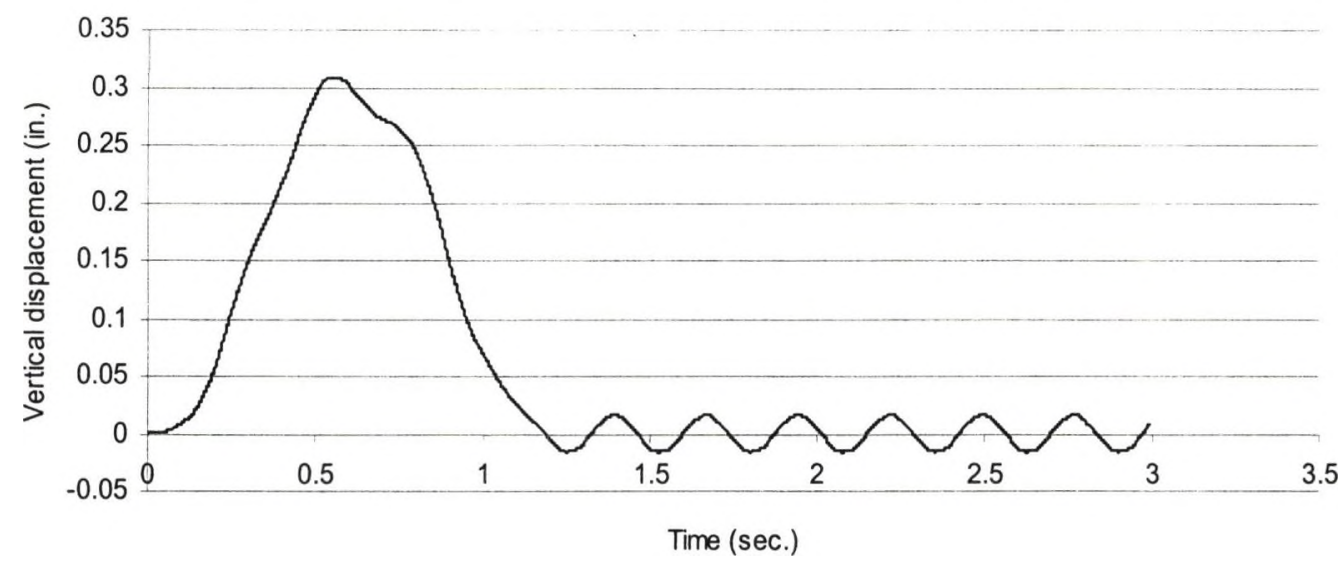

Figures C.32: Time history of the vertical displacement at the center of the T-beam bridge deck under an AASHTO HS20-44 truck with a speed of $85 \mathrm{mph}$

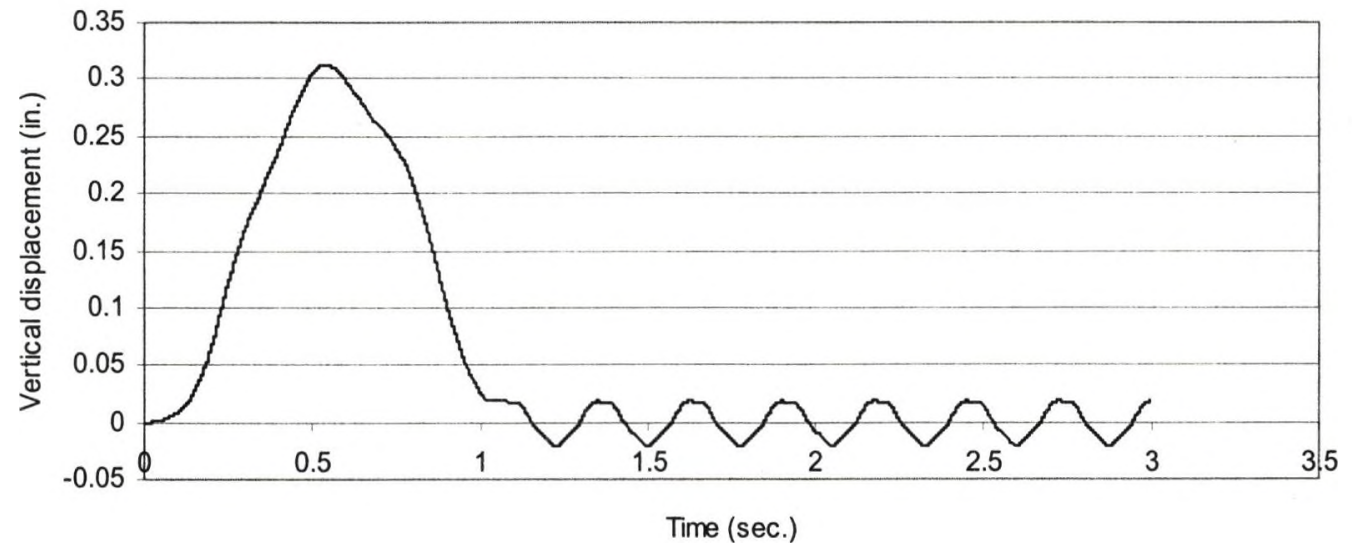

Figures C.33: Time history of the vertical displacement at the center of the T-beam bridge deck under an AASHTO HS20-44 truck with a speed of $90 \mathrm{mph}$ 


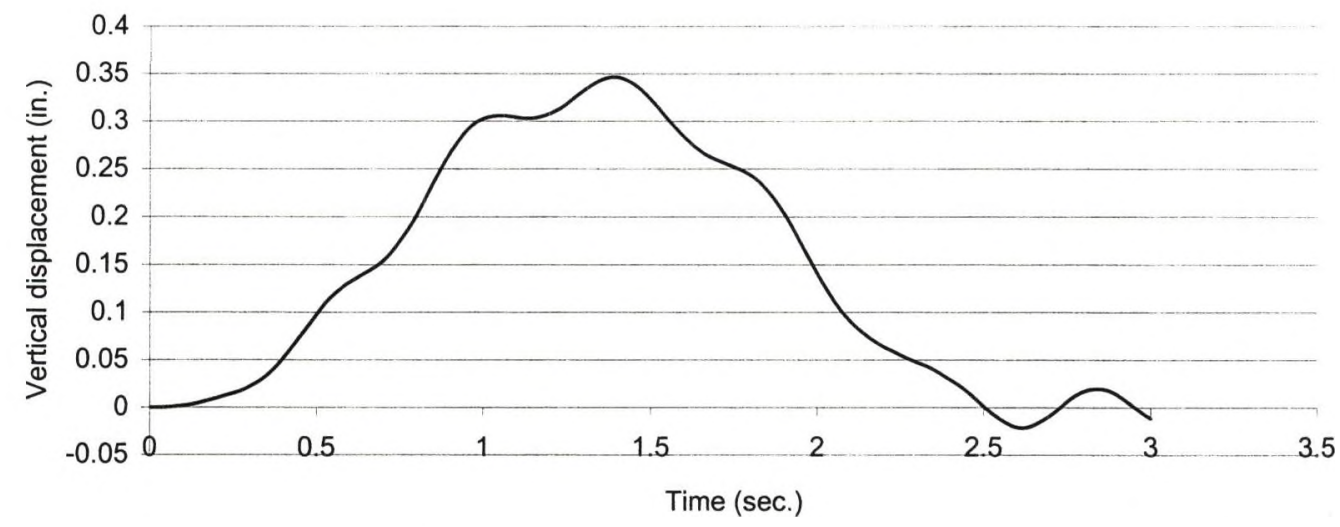

Figures C.34: Time history of the vertical displacement at the center of the T-beam bridge deck under an AASHTO HS20-44 truck with a speed of $40 \mathrm{mph}$ by FEM.

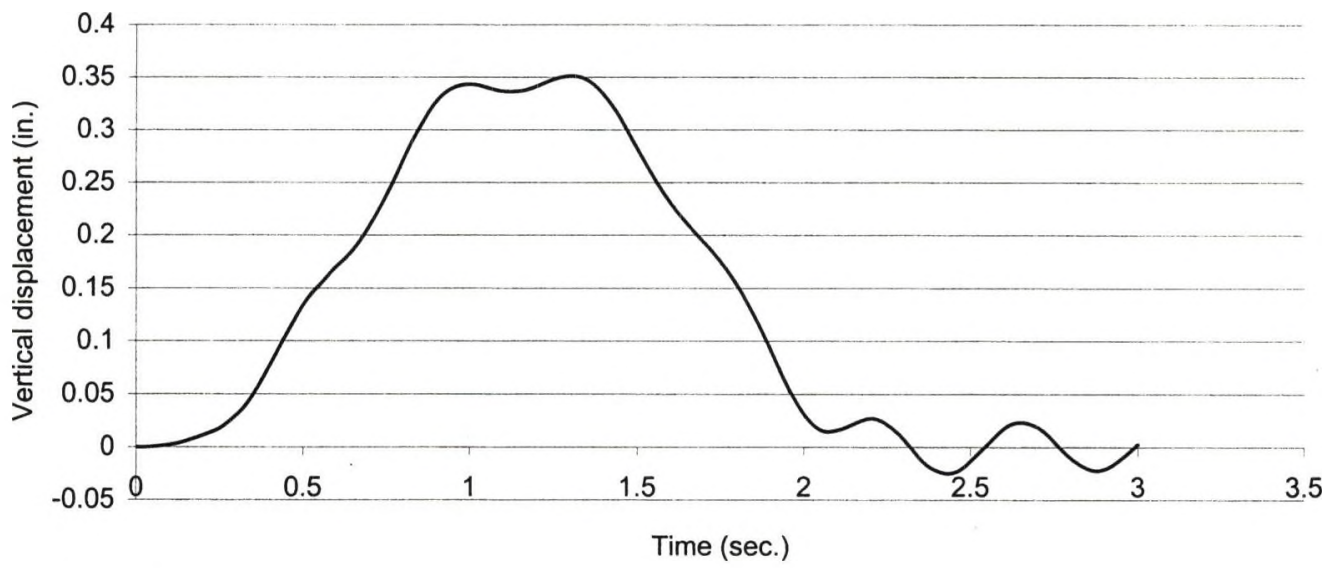

Figures C.35: Time history of the vertical displacement at the center of the T-beam bridge deck under an AASHTO HS20-44 truck with a speed of $45 \mathrm{mph}$ by FEM.

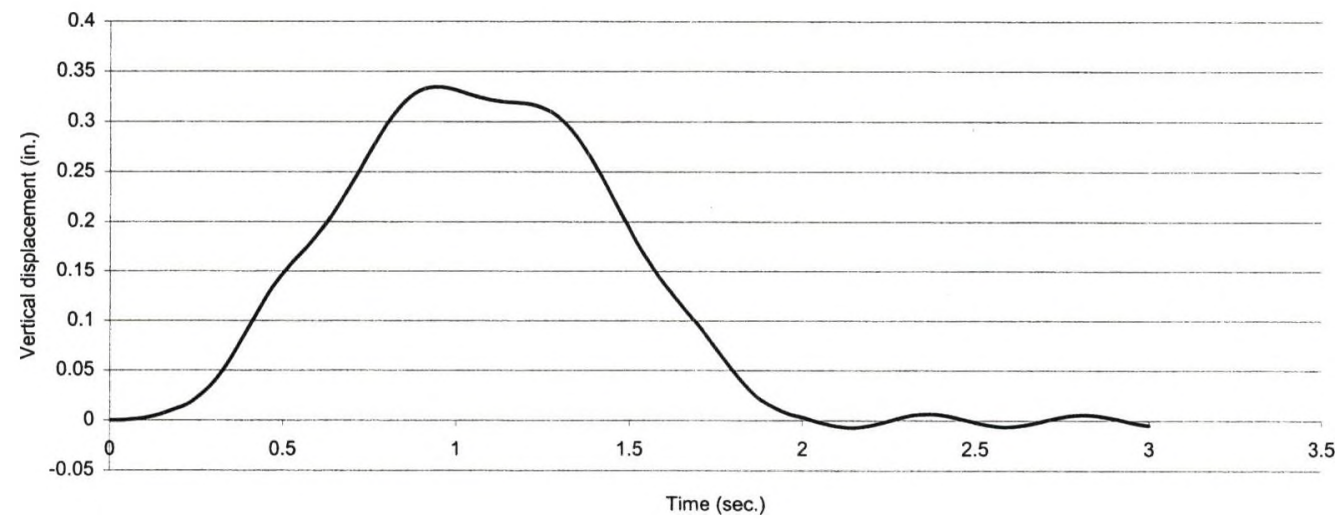

Figures C.36: Time history of the vertical displacement at the center of the T-beam bridge deck under an AASHTO HS20-44 truck with a speed of 50 mph by FEM. 


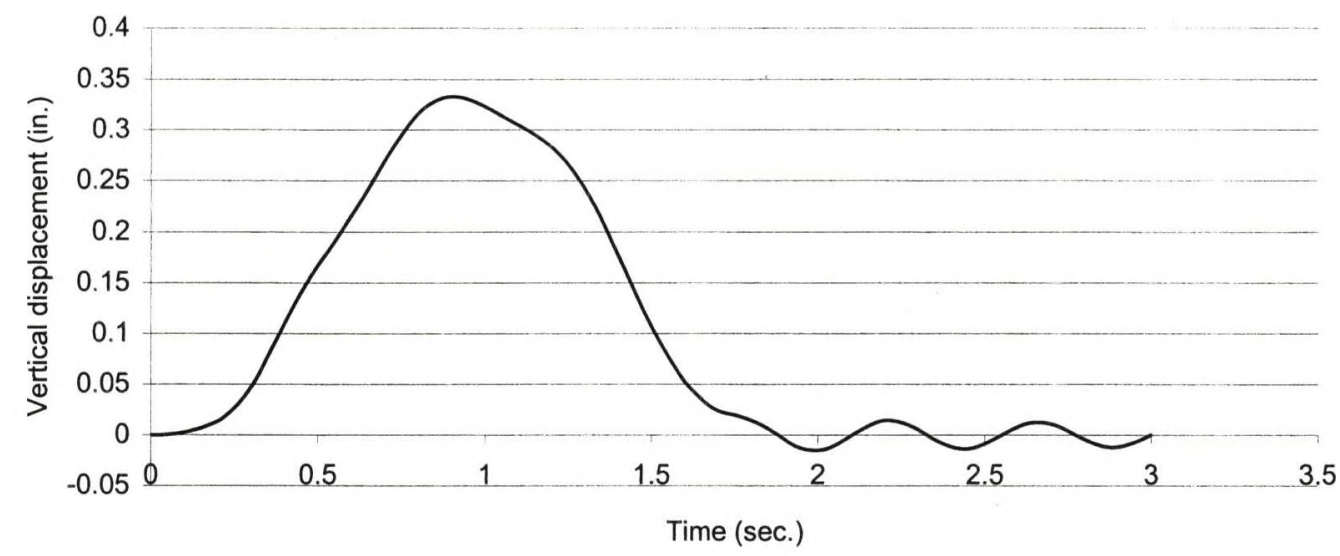

Figures C.37: Time history of the vertical displacement at the center of the T-beam bridge deck under an AASHTO HS20-44 truck with a speed of 55 mph by FEM.

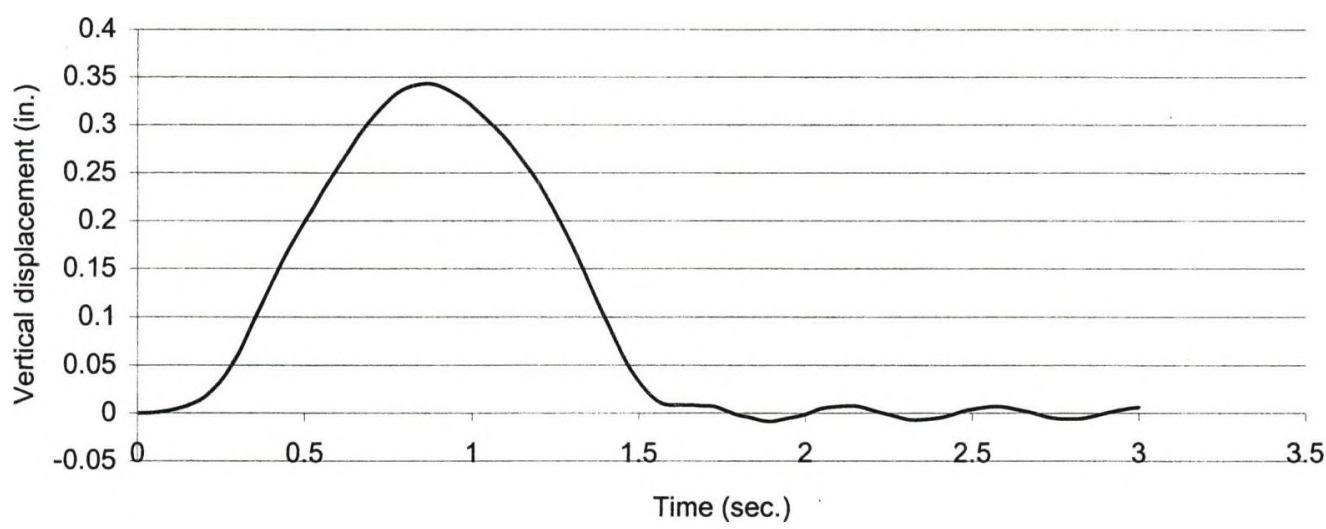

Figures C.38: Time history of the vertical displacement at the center of the T-beam bridge deck under an AASHTO HS20-44 truck with a speed of $60 \mathrm{mph}$ by FEM.

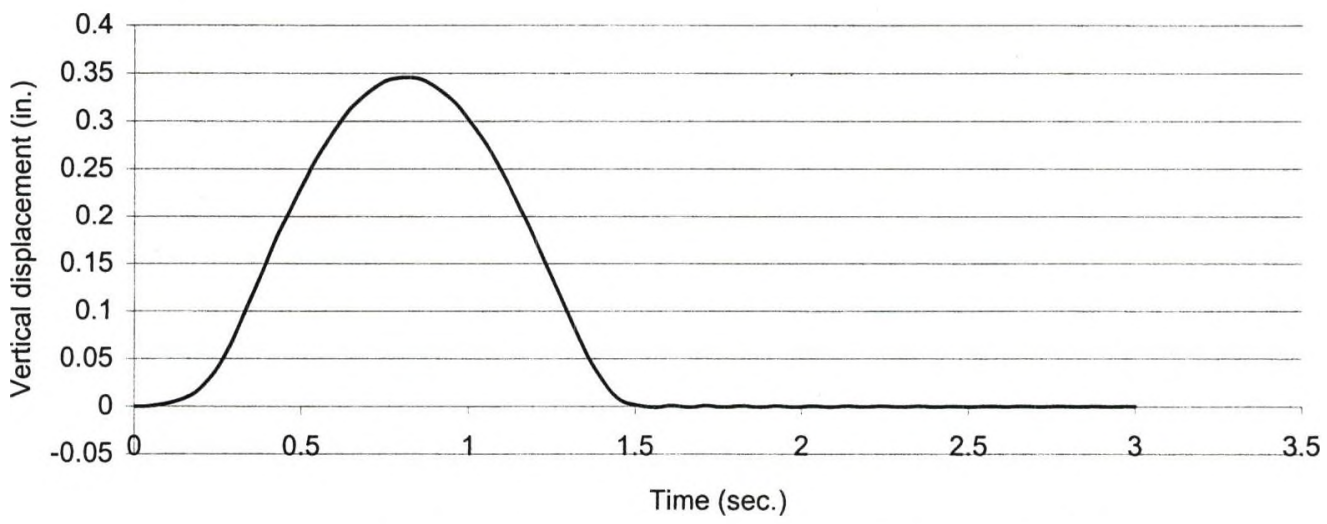

Figures C.39: Time history of the vertical displacement at the center of the T-beam bridge deck under an AASHTO HS20-44 truck with a speed of $65 \mathrm{mph}$ by FEM. 


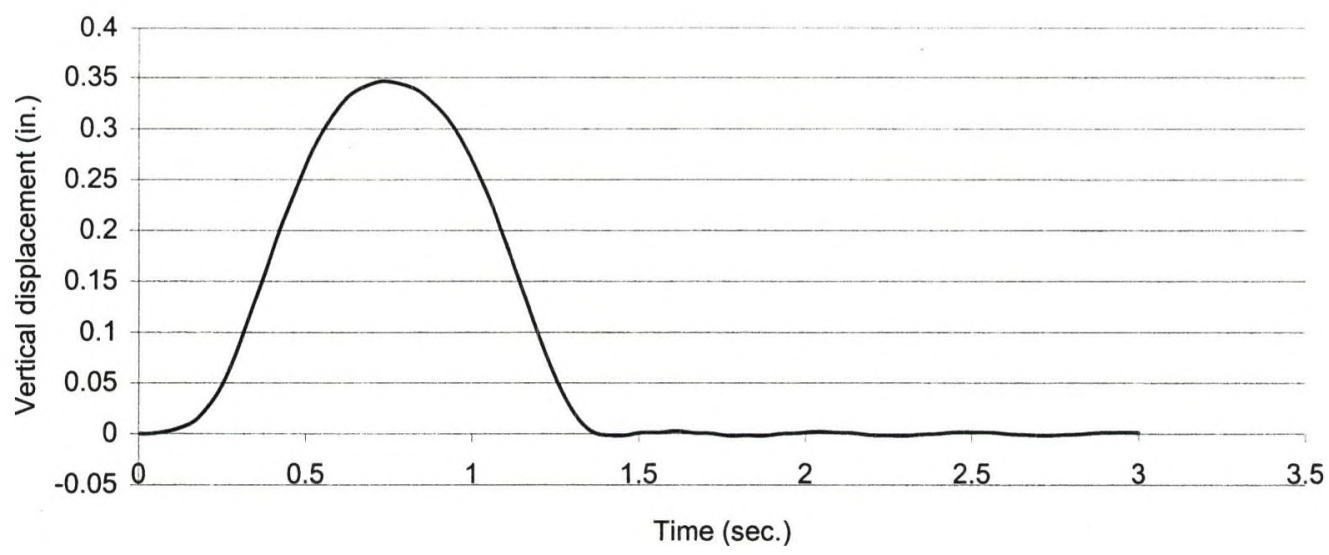

Figures C.40: Time history of the vertical displacement at the center of the T-beam bridge deck under an AASHTO HS20-44 truck with a speed of $70 \mathrm{mph}$ by FEM.

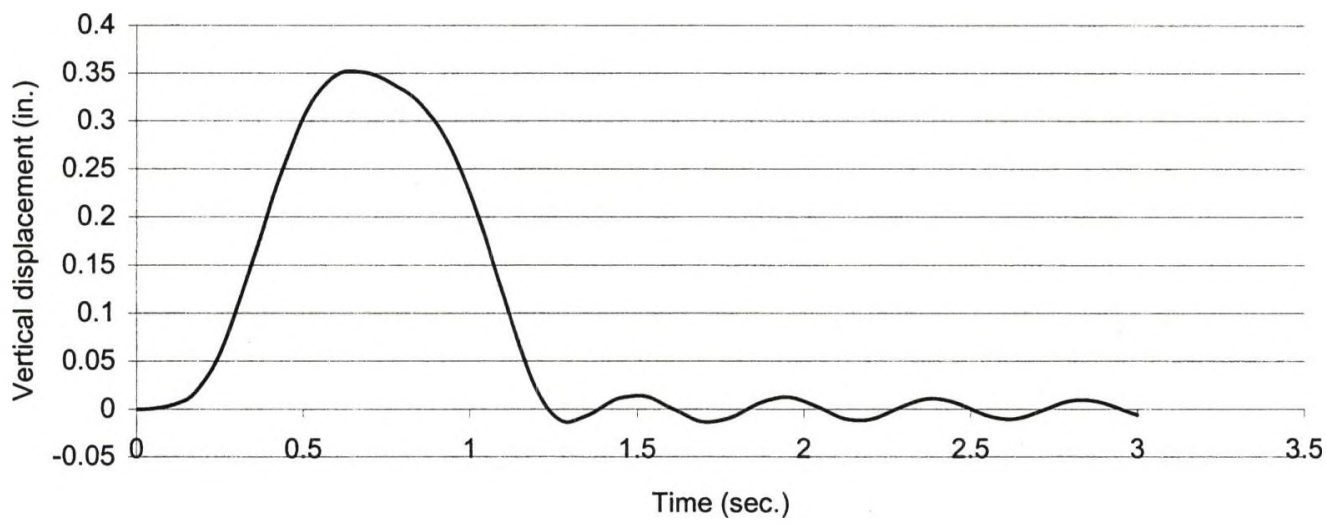

Figures C.41: Time history of the vertical displacement at the center of the T-beam bridge deck under an AASHTO HS20-44 truck with a speed of 75 mph by FEM.

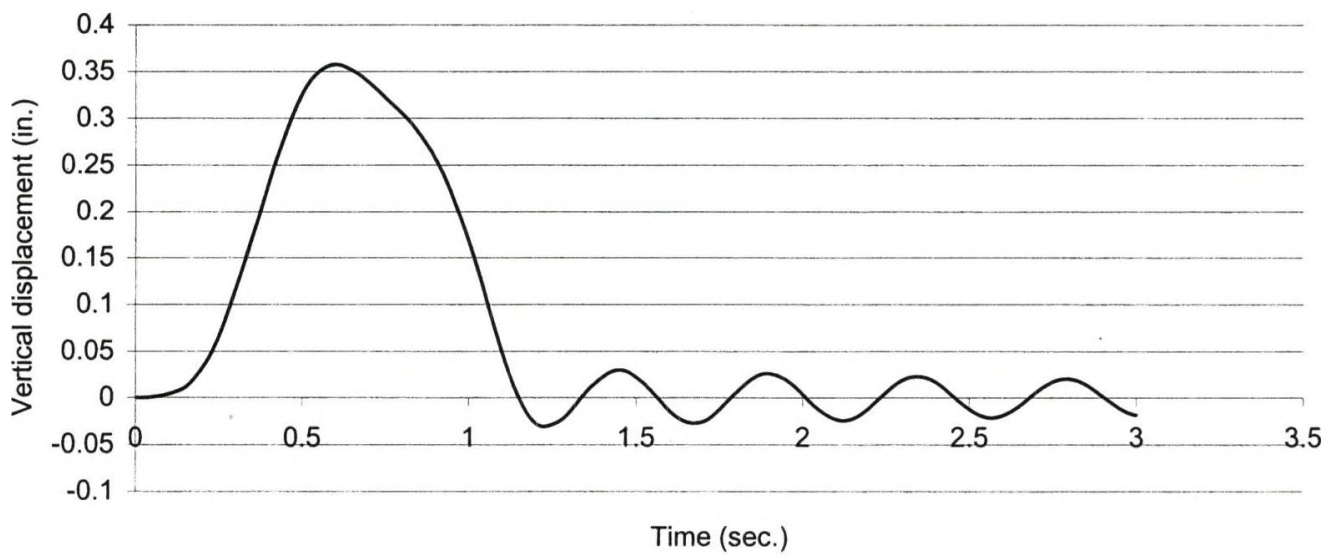

Figures C.42: Time history of the vertical displacement at the center of the T-beam bridge deck under an AASHTO HS20-44 truck with a speed of $80 \mathrm{mph}$ by FEM. 


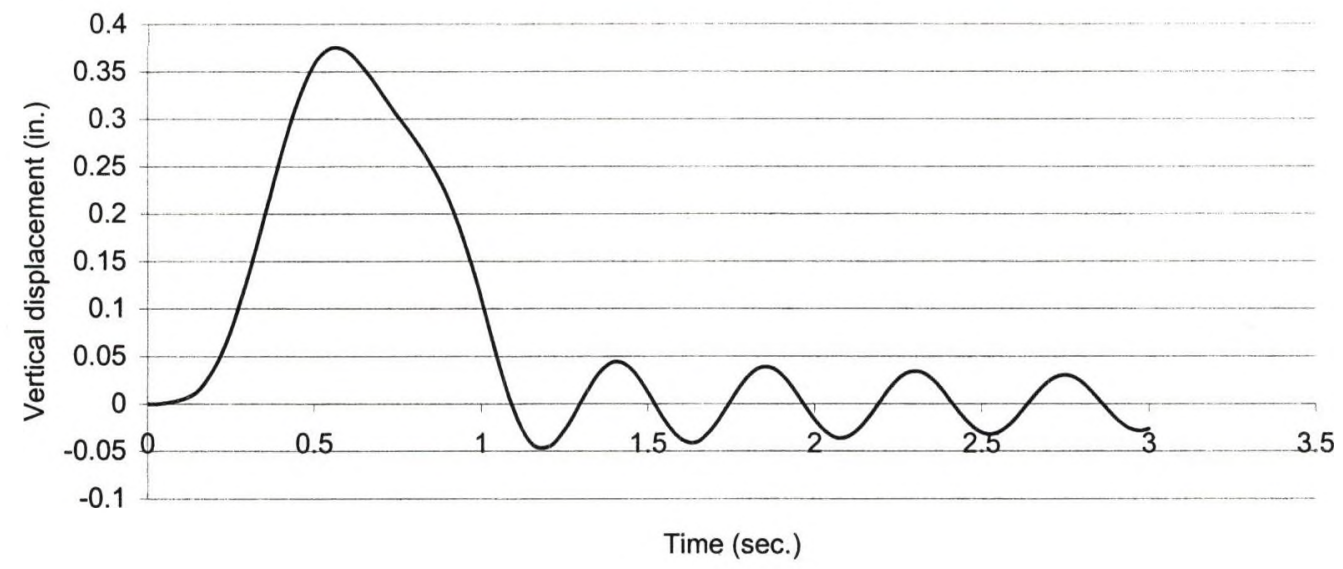

Figures C.43: Time history of the vertical displacement at the center of the T-beam bridge deck under an AASHTO HS20-44 truck with a speed of $85 \mathrm{mph}$ by FEM.

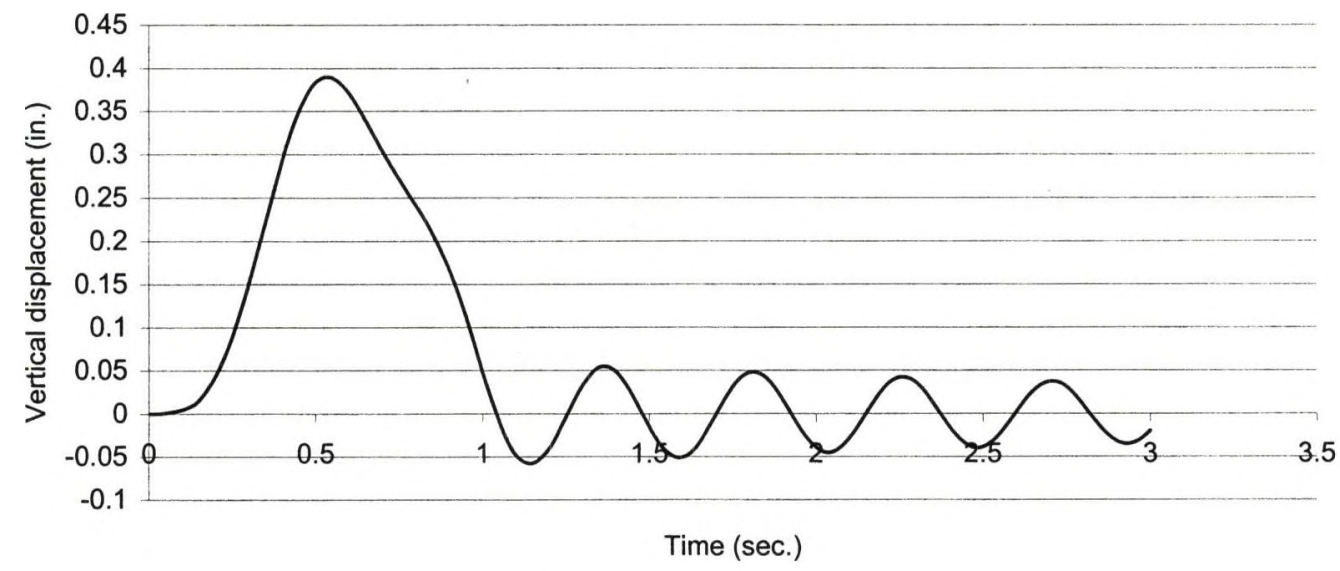

Figures C.44: Time history of the vertical displacement at the center of the T-beam bridge deck under an AASHTO HS20-44 truck with a speed of $90 \mathrm{mph}$. 


\section{REFERENCES}

1. Standard Specifications for Highway Bridges (1989). $14^{\text {th }}$ Edn. American Association of State Highway and Transportation Officials, Washington, DC.

2. EMRC NISA/Display User's Manual (1999), NISA/Display Documentation, Engineering Mechanics Research Corporation.

3. C. J. Dodds, and J. D. Robson (1973). "The Description of Road Surface Roughness." Journal of Sound and Vibration, 31(2), 175-183.

4. J. G. S.da Silva (2004). "Dynamical Performance of Highway Bridge Decks with Irregular Pavement Surface." Computers and Structures, 82, 871-881.

5. T. L. Wang, M. Shahawy, and D. Z. Huang (1993). "Dynamic Response of Highway Trucks due to Road Surface Roughness." Computers and Structures, 49(6), 1055-1067.

6. G. Jayaraman, P. Chen, and V.W. Snyder (1990). "Free Vibrations of Rectangular Orthotropic Plates with a Pair of Parallel Edges Simply Supported." Computers and Structures, 34(2), 203-214.

7. X. Q. Zhu, and S. S. Law (2002). "Dynamic Load on Continuous Multi-Lane Bridge Deck from Moving Vehicles." Journal of Sound and Vibrations, 251(4), 697-716.

8. $\quad$ Fryba, L. (1972). "Vibrations of Solids and Structures under Moving Loads." Noordhoff International, Groningen, The Netherlands. 
9. D. P. Thambiratnam, and G. H. Brameld (2000). "Experimental Analysis of an Asymmetric Reinforced Concrete Bridge under Vehicular Loads.” Structural Engineering and Mechanics, 9(1), 17-35.

10. R. P. Labarre, R. T. Forbes, and S. Andrew (1969). "The Measurement and Analysis of Road Surface Roughness." Motor Industry Research Association Report No. 1970/5.

11. Robert K. Otnes, and Loren Enochson (1972). "Digital Time Series Analysis." John Wiley, New York.

12. S. Timoshenko, and S. Woinowsky-Krieger (1959). "Theory of Plates and Shells." 2 ${ }^{\text {nd }}$ Edn. McGraw-Hill, New York.

13. Design Manual for Orthotropic Steel Plate Deck Bridges (1963). American Institute of Steel Construction, New York.

14. D. J. Gorman (1982). "Free Vibration Analysis of Rectangular Plates." Elsevier, New York.

15. Jong-Shyong Wu, Ming-Ling Lee, and Tser-Shyong Lai (1987). "The Dynamic Analysis of a Flat Plate under a Moving Load by the Finite Element Method." International Journal for Numerical Methods in Engineering, 24, 743-762.

16. A. R. Cusens, and R. P. Pama (1975). "Bridge Deck Analysis." John Wiley, New York. 